\title{
Illustrated identification guide to the Nordic aphids feeding on Conifers (Pinophyta) (Insecta, Hemiptera, Sternorhyncha, Aphidomorpha)
}

\author{
Anders Christian ALBRECHT \\ Finnish Museum of Natural History - University of Helsinki, Zoology, \\ P. Rautatiekatu 13, Helsinki FI-00014, Finland. \\ Email: anders.albrecht@helsinki.fi
}

urn:lsid:zoobank.org:author:3835E475-B3F6-4965-A4E6-A3AE55659C86

\begin{abstract}
Keys and diagnoses of North European aphids (Hemiptera, Aphidoidea) feeding on conifers are given, including species from nearby areas of Central and Western Europe, based on live and freeze-dried material. Externally visible informative characters, such as body shape, colours, wax coating, and pigmentation pattern are utilized, in addition to characters traditionally used in the literature. Rich illustrations with photographs of live colonies and freeze-dried specimens, supported by drawings where needed, are presented. The combination of colour images and diagnoses, utilizing easily observed characters, allows the identification of a large number of species already in the field, and many more at home with the aid of a stereo microscope. Host plant relationships and aphid-ant associations are presented.
\end{abstract}

Keywords. Nordic aphids on conifers, identification keys, colour images, Adelgidae, Cinarinae.

Albrecht A.C. 2017. Illustrated identification guide to the Nordic aphids feeding on Conifers (Pinophyta) (Insecta, Hemiptera, Sternorhyncha, Aphidomorpha). European Journal of Taxonomy 338: 1-160. https://doi.org/10.5852/ ejt.2017.338

\section{Introduction}

This is the second article presenting the Nordic Aphidomorpha. The purpose of this work is to display them in a way that is closer to their natural appearance than how aphid handbooks traditionally present them. This is intended to facilitate the study of this fascinating group of insects. The present study focuses on conifer-feeding aphids of Finland and Scandinavia, including Iceland, Greenland, The Faroes and Svalbard. Most of the species occurring in adjacent parts of north-eastern Europe, the Baltic region and northern central Europe, including the British Isles are also covered. For a more complete introduction, see Albrecht (2015).

The aphid fauna on conifers is quite different from that of angiosperms. The Aphidinae are clearly under-represented in favour of Adelgidae and Lachninae, and to some extent Eriosomatinae (family 
Aphididae). These taxa form three distinct identities regarding life cycles and feeding habits. Adelgids are characterised by being oviparous in all female morphs, by a two-year life cycle (when holocyclic) with a gall-forming generation on spruce alternating with free-living generations on fir, larch, pine etc. They hibernate as nymphs and can accordingly be found any time of the year. Even if very small, the overwintering individuals are fairly easy to identify, with a good lupe even in the field.

The Lachninae, on the other hand, are all monoecious and have a one-year life cycle, hibernating as eggs on needles, in bark crevices, between cone scales and so on. Regardless of their often large size, some species, even common ones, are problematic and clearly in need of revision. Cinara pini (Macquart, 1819 ) and allied species, as well as the highly variable C. pinea (Mordvilko, 1895) (as here interpreted), deserve special attention.

Their wax and colour patterns are often conspicuous, so studies of fresh material are of utmost importance. Freeze-dried material is a very good complement. A couple of points need, however, to be taken into account when comparing fresh and dry material: freeze-dried specimens lack the transparency of live material; also, colouration due to body fluid will be opaque and much paler, wax pattern less obvious (although not lost), and sclerites will stand out very distinctly. Moreover, assessing the host relationships of many Cinara species is far from straight forward. Particularly with some large species (e.g., Cinara (Cinara) piceae (Panzer, 1801), C. confinis Koch, 1856, C. nuda Mordvilko, 1895) as adults are easily disturbed and tend to fall to the ground in windy weather, whereupon they climb up on anything vertical, and may be found on trees not serving as regular hosts. Binazzi \& Scheurer (2009) gave an informative account on honeydew-producing conifer Lachnidae in Europe. The British species of conifer lachnids are extensively treated by Carter \& Maslen (1982).

The eriosomatines feeding on conifers are a chapter of their own. They are all dioecious species with broad-leaved trees or shrubs as primary hosts, and feed on thin, usually mycorrhizal, roots of conifers. They can be found beneath the moss layer under conifers and are usually revealed by their white wax exudates, which is very helpful given their small size. Many of them (if not all) may hibernate here, and even stay here for many years. For more details, see Albrecht (2015).

Mindarinae is a small subfamily confined to conifers, whereas the large Aphidinae is represented by just a few species, predominantly polyphags occasionally found on conifers.

\section{Material and methods}

The host records and distribution data are according to Blackman \& Eastop (1994, 2006, 2016), Heie (1980, 1986, 1992, 1994, 1995, 2004), Holman (2009) and the database of the Finnish Expert Group on Hemiptera (http://biolcoll.utu.fi/hemi/tyoryhma/tyoryhma_eng.htm), comprising most of the aphid data recorded from Finland, with additional records from adjacent countries. The photographs and drawings are original, unless otherwise stated. My own records of hosts and attendant ants are marked with asterisks $(*)$ in the diagnoses and in the host and ant tables (Appendices 1 and 2), as are records by Osmo Heikinheimo and other Finnish collectors whenever it has been possible to confirm the relationships (e.g., from Heikinheimo's field notes). The plant nomenclature follows Karlsson \& Agestam (2014). For the part of the attendant ants, I have not had the opportunity to scan the literature, so the ant lists are more or less completely relying on my own observations. My ant identifications and nomenclature are based on Douwes et al. (2012) and Czechowski et al. (2012). The aphid nomenclature and classification in this article follows Favret (2016) and Favret et al. (2015). Whenever available, live and freeze-dried material is used for the diagnoses, in conjunction with the literature. For geographical and phenological records from Finland, see Albrecht (2010). 
ALBRECHT A.C., Identification guide to Nordic aphids on conifers

The keys, instead of being dichotomous, mainly consist of multi-character diagnoses and synopses to entry points grouping the diagnoses into sections sharing one or a few easily observed characters. Together with the photographs and drawings the data given in the diagnoses should in most cases be enough to allow correct identifications, or when that is not the case, to give sufficient reason to doubt the results and seek additional information elsewhere in the literature.

Some terms and abbreviations used in this paper (for more, see e.g., Albrecht 2015; Blackman \& Eastop 2016).

$\begin{array}{lll}\text { al. } & =\text { alata, alate, winged } \\ \text { anholocycle } & = & \text { incomplete life cycle, asexual generations only } \\ \text { ant. } & = & \text { antenna } \\ \text { fundatrix } & = & \text { female hatched from a fertilized egg (often gall-inducer) } \\ \text { gallicola } & = & \text { female inhabiting or emanating from gall } \\ \text { holocycle } & = & \text { complete life cycle with alternating sexual and asexual generations } \\ \text { juv. } & = & \text { juvenile, nymph } \\ \text { neosistens } & = & \text { first larval instar of overwintering female on secondary host (sistens) } \\ \text { primary host } & = & \text { main host of host-alternating species, where the sexual reproduction takes place } \\ \text { pseudofundatrix } & = & \text { gall-inducer hatched from unfertilized egg (in some anholocyclic Adelgidae) } \\ \text { RIV } & = & \text { rostral segment } 4 \\ \text { RV } & = & \text { rostral segment } 5 \\ \text { RIV+V } & = & \text { rostral segments } 4 \text { and } 5 \text { combined } \\ \text { secondary host } & = & \text { intermediate host of host-alternating species where only asexual reproduction } \\ & & \text { takes place }\end{array}$

The presence of the species in the Nordic countries is based on Heie $(1980,1986,1992,1994,1995$, 2004) and my own databases. The countries are denoted by letters as follows:

$$
\begin{aligned}
\mathrm{D} & =\text { Denmark } \\
\mathrm{F} & =\text { Finland } \\
\mathrm{Fa} & =\text { Faroes } \\
\mathrm{Gr} & =\text { Greenland } \\
\mathrm{I} & =\text { Iceland } \\
\mathrm{N} & =\text { Norway } \\
\mathrm{S} & =\text { Sweden } \\
\mathrm{Sv} & =\text { Svalbard }
\end{aligned}
$$

\section{Results}

Phylum Arthropoda von Siebold, 1848

Class Insecta Linnaeus, 1758

Order Hemiptera Linnaeus, 1758

Suborder Sternorrhyncha Amyot \& Serville, 1834

Infraorder Aphidomorpha Becker-Migdisova \& Aizenberg, 1962

\section{Synoptic key and diagnoses for aphids on conifers}

Aphids on spruce (Picea) Key A, p. 4 
Aphids on larch (Larix)

Key C, p. 23

Aphids on hemlock and Douglas fir (Tsuga and Pseudotsuga)

Key D, p. 27

Aphids on pine (Pinus)

Key E, p. 29

Aphids on Cupressaceae

Key F, p. 40

\section{Key A. Aphids on spruce (Picea)}

\section{Synopsis}

A1 (A2)

Dichotomous key to galls on Picea p. 5

A2 (A1)

Aphids on Picea

A3 (A15)

On aerial parts of Picea

Siphunculi absent; adults with sclerotised ovipositor. Apterae less than $1.2 \mathrm{~mm}$, antennae at most 3 -segmented Adelgidae Annand, 1928 A5

Abdomen (all morphs) with 4 pairs of evident spiracles (on abd. 2-5). Gall (when present) with interconnected chambers Pineus Shimer, 1869: p. 6

A6 (A5) Abdomen (all morphs) with 5 pairs of evident spiracles (on abd. 2-6). Gall with separate chambers

A7 (A8) Wax glands of overwintering larvae with thin-walled facets secreting solid wax filaments; galls without protruding needles, hair or waxy bloom

Adelges (Aphrastasia) Börner, 1909 and A. (Dreyfusia) Börner, 1908) p. 8

A8 (A7) Wax glands of overwintering larvae with double-walled facets secreting glossy, stiff, straight or curved tubes of wax; galls usually with protruding needles and hairs or waxy bloom

A9 (A10) Overwintering larvae (where known): dorsal sclerites bearing single larger wax pores ringed by small glands of irregular shape. Al. gallicola: ant. IV and V often with broad, indistinct basal articulation Adelges (Adelges) Vallot, 1836 p. 9

A10 (A9) Overwintering larvae: dorsal sclerites with groups of similar-sized ring-like wax pores. Al. gallicola with basal articulation of ant. IV and V narrower Adelges (Gilletteella Börner, 1930 and Sacciphantes Curtis, 1844) p. 11

A11 (A4) Siphunculi present; adults without sclerotised ovipositor. Apterae rarely less than $1.2 \mathrm{~mm}$; antennae at least 4-segmented

Aphididae Latreille, 1802 A12

A12 (A13 A14) Siphunculi elongate, tubular ................. Elatobium Mordvilko, 1914, Aulacorthum Mordvilko, 1914, Neomyzus van der Goot, 1915 and Aphis Linnaeus, 1758 p. 12

A13 (A12 A14) Siphunculi present as pores not raised from body surface

Mindarus Koch, 1857 p. 14

A14 (A12 A13) Siphunculi present as large pores elevated on obtuse cones

Cinara Curtis, 1835 p. 14

A15 (A3) Aphids on roots of Picea A16

A16 (A17) Apterae 2-6 mm, with brown, green or blackish colours; siphunculi present as pores elevated on cones. On thick roots, usually ant-attended

Cinara Curtis, 1835 p. 17

A17 (A16) Apterae 0.5-2 mm, whitish, pale green or yellowish, exuding abundant white wax wool; siphunculi absent. On thin mycorrhizal root ends, not attended by ants 
A1 (A2) Dichotomous key to galls on Picea

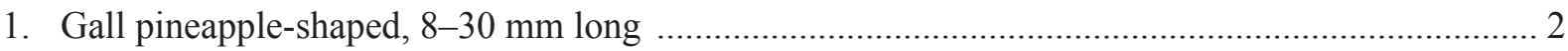

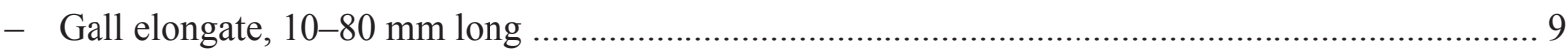

2. Gall with conspicuous needle-bearing shoot apically, bright, often with red or purple scale margins; finely pubescent, without wax dusting 3

- Needle-bearing apical shoot inconspicuous or absent; thinly wax-dusted, not pubescent ............... 4

3. Galls usually gregarious, often along with galls from preceding years, opening in August or early September Adelges (Sacciphantes) abietis (Linnaeus, 1758) p. 11

- Galls usually solitary, opening in July or early August Adelges (Sacciphantes) viridis (Ratzeburg, 1843) p. 12

4. On Picea orientalis. Gall globular, red or pink when young, later on greenish with red or purple at bases and tips of scales Adelges (Dreyfusia) nordmannianae (Eckstein, 1890) p. 8 and Adelges (Dreyfusia) merkeri (Eichhorn, 1957) p. 9

- Usually on Picea abies or P. sitchensis. Gall dull green, brownish or pale yellow, often with pinkish tinge

5. Gall compact, cone-like, without protruding needles, dull green as young, later on reddishbrown Adelges (Aphrastasia) pectinatae (Cholodkovsky, 1888) p. 8

- Gall with protruding needles, pale yellow, often with pinkish tinge; at maturity turning purple ..... 6

6. Galls solitary 7

- Galls usually gregarious, often along with galls from preceding years 8

7. Galls opening in June-July; gallicolae blackish, secreting only little wax

- Galls opening late June-September; gallicolae secrete abundant wax Adelges (Adelges) laricis Vallot, 1836 p. 9 Adelges (Adelges) tardoides (Cholodkovsky, 1911) p. 10

8. Galls opening in June-July; gallicolae dark red, secreting only little wax

Adelges (Adelges) lapponicus (Cholodkovsky, 1889) p. 9

- Galls opening August-September; gallicolae blackish brown, secreting abundant wax Adelges (Adelges) tardus (Dreyfus, 1888) p. 10

9. Usually on Picea sitchensis. Needles from scale-like widened bases strongly protruding. Chambers separate with green, pink or red openings Adelges (Gilletteella) cooleyi (Gillette, 1907) p. 11

- Gall with interconnected chambers and more or less decumbent needles 10

10. Gall with needles much paler than the normal new growth

Pineus (Pineus) similis (Gillette, 1907) p. 6

- Needles in gall not paler than normal new growth .11

11. Gall usually bent and distorting shoot tip, with more protruding needles, often directed to one side; usually on Picea abies Pineus (Pineus) cembrae (Cholodkovsky, 1888) p. 7

- Gall short and more or less straight with decumbent needles evenly distributed to all sides, usually on Picea orientalis Pineus (Pineus) orientalis (Dreyfus, 1889) p. 7 


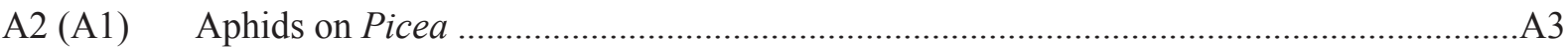

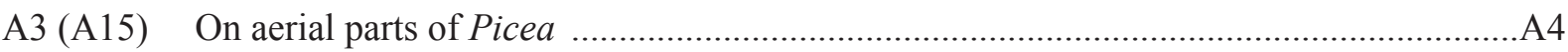

A4 (A11) Siphunculi absent; adults with sclerotised ovipositor. Apterae less than $1.2 \mathrm{~mm}$, antennae at most 3-segmented. Adelgidae Annand, 1928 A5

Superfamily Adelgoidea Annand, 1928

Family Adelgidae Annand, 1928

\section{Diagnosis}

Gall-inducing species belong here. Holocyclic species with 2-year life cycle (see Albrecht 2015; Blackman \& Eastop 1994). The adelgids are not attended by ants.

A5 (A6) Abdomen (all morphs) with 4 pairs of evident spiracles (on abd. 2-5). Gall (when present) with interconnected chambers

Pineus Shimer, 1869

Genus Pineus Shimer, 1869

Subgenus Pineus Shimer, 1869

Pineus (Pineus) pineoides (Cholodkovsky, 1907)

Fig. 1

\section{Diagnosis}

Apterae (one morph only) $0.6-0.8 \mathrm{~mm}$, yellowish-grey to red-brown, dorsoventrally flattened, waxcovered. Head and prothorax fused, strongly sclerotised. Alatae unknown. Anholocyclic on Picea. Freeliving in bark crevices, under scales, etc. on shaded parts of trunk and branches; one summer and one winter generation per year.

\section{Recorded hosts}

Pinaceae: Picea abies*, omorika.

\section{Distribution}

F.

Pineus (Pineus) similis (Gillette, 1907)

\section{Diagnosis}

In or near galls on shoots. Both apterous (uniquely) and al. gallicolae produced. Al. gallicolae 1-2 mm, dark reddish brown. Anholocyclic, monoecious on a wide range of Picea species. In North America Picea abies, glauca, engelmanni, mariana, pungens, rubens; in Britain colonising P. sitchensis (Carter 1976). Pseudofundatrices overwinter in the second or third instar under bud scales. In spring they cause rather loose galls 1-4 cm long, with the constituent needles much paler than the normal new growth. As the gall matures the shoot and needles become brown and twisted giving it a ragged appearance. Apterous gallicolae oviposit within the galls, but may also be found outside the galls, laying eggs in small tufts of 
wax on the stem. Al. gallicolae, 1.0-2.1 mm, dark reddish-brown, fly in June-July to recolonise spruce needles, laying eggs in white wax wool. These apparently develop into the overwintering pseudofundatrix generation. Diagnosis based on Blackman \& Eastop (2006).

\section{Recorded hosts (in Britain)}

Pinaceae: Picea sitchensis.

\section{Distribution}

North America, introduced to Europe (Britain).

Pineus (Pineus) cembrae (Cholodkovsky, 1888)

Fig. 2

\section{Diagnosis}

In or near galls on shoots. Abdomen of overwintering juv. fundatrix with spinal and pleural wax glands on all tergites. Adult fundatrix with spinopleural glands on terg. 1-3, 1-4 or 1-5, and well-developed marginal glands on terg. Al. gallicola $1.5-1.7 \mathrm{~mm}$, dark red with abundant wax; primary rhinarium on ant. V not extending more than half-way around the segment. Gall $4-5 \mathrm{~cm}$ long, straight or bending the shoot tip and concentrating the protruding needles to one side. Holocyclic and dioecious, alternating between Picea and Pinus (see p. 30). Partly anholocyclic on Pinus.

\section{Recorded primary hosts}

Pinaceae: Picea abies (usually), glehnii, jezoensis, koraiensis, orientalis.

\section{Distribution}

F S.

Pineus (Pineus) orientalis (Dreyfus, 1889)

Figs 3-4

\section{Diagnosis}

In or near galls on shoots. Al. gallicola: $1.5-2.3 \mathrm{~mm}$, primary rhinaria on ant. segment $\mathrm{V}$ extending more than half-way around the segment, usually not occupying more than half the length of the segment. Gall terminal, shorter (about $3 \mathrm{~cm}$ ) and usually straighter than in $P$. cembrae, with needles more or less decumbent and distributed to all sides. Holocyclic and dioecious, alternating between Picea and Pinus (see also p. 30).

\section{Recorded primary hosts}

Pinaceae: Picea jezoensis, orientalis*, pungens, torano, spp. (but not abies!).

\section{Distribution}

D F S.

\section{Diagnosis}

Pineus (Pineus) strobi (Hartig, 1837)

Anholocyclic on Pinus strobus (see p. 31). Al. sexuparae lay eggs on of Picea mariana, but no sexuales are produced (Blackman \& Eastop 1994). 
A6 (A5) Abdomen (all morphs) with 5 pairs of evident spiracles (on abd. 2-6). Gall with separate chambers

A7 (A8) Wax glands of overwintering larvae with thin-walled facets secreting solid wax filaments; galls without protruding needles, hair or waxy bloom

Adelges (Aphrastasia Börner, 1909 and Dreyfusia Börner, 1908)

Genus Adelges Vallot, 1836

Subgenus Aphrastasia Börner, 1909

Adelges (Aphrastasia) pectinatae (Cholodkovsky, 1888)

Fig. 5

\section{Diagnosis}

Fund. juv. with spinal, pleural and marginal wax pore plates. Al. gallicola reddish brown, wax glands on head and prothorax with many distinct facets; rhinaria on ant. III, IV and V occupying more than half segment length. Gall compact, cone-like, dull green as young, later reddish-brown, 10-17 mm. Holocyclic and dioecious, alternating between Picea and Abies (see also p. 20). In Finland A. pectinatae seems to be anholocyclic on Abies.

\section{Recorded primary hosts}

Pinaceae: Picea abies, glauca, glehnii, jezoensis, koraiensis, sitchensis.

\section{Distribution}

F N S.

Subgenus Dreyfusia Börner, 1908

Adelges (Dreyfusia) nordmannianae (Eckstein, 1890)

Fig. 6

\section{Diagnosis}

Fund. juv. only with spinal and marginal wax pore plates; wax pore plates on inner margins of meso- and metathoracic spinal sclerites with numerous small pits arranged in 2-4 areas of rather rounded shape. Al. gallicola: 1.1-2.3 mm, wax glands on head and prothorax of granular appearance, without or with a few indistinct facets. Galls 7-15 mm, terminal, globular, red when young (resembling strawberries), later greenish with red or purple at bases and tips of scales. Holocyclic and dioecious, alternating between Picea orientalis and Abies (see also p. 20).

\section{Recorded primary hosts}

Pinaceae: Picea orientalis (usually), abies, omorika.

\section{Distribution}

D N S. 
Fig. 7

\section{Diagnosis}

Fund. juv. only with spinal and marginal wax pore plates; wax pore plates on inner margins of meso- and metathoracic spinal sclerites divided into 3-4 often angular areas with relatively large pits of irregular shape. Al. gallicola: wax glands on head and prothorax of granular appearance, without or with a few indistinct facets. Galls like those of $A$. nordmannianae but are usually larger (7-22 $\mathrm{mm}$ ) and open in June-July. Holocyclic and dioecious, alternating between Picea orientalis and Abies (see also p. 21).

\section{Recorded primary hosts}

Pinaceae: Picea orientalis.

\section{Distribution}

S.

A8 (A7) Wax glands of overwintering larvae with double-walled facets secreting glossy, stiff, straight or curved tubes of wax; galls usually with protruding needles and hairs or waxy bloom

A9 (A10) Overwintering larvae (where known): dorsal sclerites bearing single larger wax pores ringed by small glands of irregular shape. Al. gallicola: ant. IV and V often with broad, indistinct basal articulation Adelges (Adelges) Vallot, 1836

Subgenus Adelges Vallot, 1836

Adelges (Adelges) laricis Vallot, 1836

Figs 8-9

\section{Diagnosis}

Fundatrix about $2.5 \mathrm{~mm}$, blue-green with long wax filaments. Al. gallicolae 1-2 mm, blackish, wax glands poorly developed, premarginal glands absent, wax wool sparsely produced; head with 4 separate wax gland plates; ant. III shorter than IV. Galls $5-15 \mathrm{~mm}$, pale yellowish with a waxy texture, often with a slight pinkish tinge, widely dispersed, usually singly on their branch; terminal or with very restricted plant growth beyond the gall; chambers not completely closed: nymphs often seen on outer gall surface. Galls opening in June-July. Holocyclic and dioecious, alternating between Picea and Larix (see also p. 24). Mixed spruce forests, rarely in moist or shady positions, also on solitary trees.

\section{Recorded primary hosts}

Pinaceae: Picea abies*, glauca, koraiensis, mariana, orientalis, pungens, sitchensis.

\section{Distribution}

D F N S.

Adelges (Adelges) lapponicus (Cholodkovsky, 1889)

\section{Diagnosis}

Similar to A. laricis. Al. gallicolae dark red, $1.5 \mathrm{~mm}$, laying eggs hardly covered with wax. Anholocyclic on Picea. Mixed spruce forests. Usually flying only a short distance. Galls normally many together, 
along with galls from preceding years, opening in June-July. Gallicolae dark red, secreting only little wax.

\section{Recorded hosts}

Pinaceae: Picea abies*, glauca*, jezoensis, mariana, pungens, sitchensis.

\section{Distribution}

F.

Adelges (Adelges) tardoides (Cholodkovsky, 1911)

\section{Diagnosis}

In most respects similar to A. laricis. Al. gallicolae: ant. III longer than IV; glands (including premarginal) developed, abundant wax wool produced. Holocyclic and dioecious, alternating between Picea and Larix (see also p. 25). Galls 5-15 mm, widely dispersed, usually singly on their branch, opening late June-September. Gallicolae emerge from late July to August; secreting abundant wax.

\section{Recorded primary hosts}

Pinaceae: Picea abies.

\section{Distribution}

Europe. Not yet in the Nordic countries.

\section{Adelges (Adelges) tardus (Dreyfus, 1888)}

Figs 10-14

\section{Diagnosis}

Overwintering pseudofundatrix nymphs $0.3-0.4 \mathrm{~mm}$, blackish with sparse, glossy grey, stiff, almost straight wax filaments. Al. gallicolae: 1-2 mm; ant. III longer than IV; glands (including premarginal) developed, blackish with reddish brown abdomen exuding abundant fine wax wool. They remain in the vicinity or their gall, laying eggs covered with abundant wax. Galls as in A. laricis, but gregarious, along with galls from preceding years, opening in August-September. Gall chambers not completely closed: nymphs can frequently be seen on the outer surface. Gallicolae blackish, secreting abundant wax. Anholocyclic on Picea. Usually on trees in rather open position: clearings, forest margins, solitary spruces and spruce hedges. Galls frequently intermixed with galls of Adelges abietis. Overwintering pseudofundatrices seem to prefer the surface of buds, not their bases as in A. abietis (according to my own records from Finland).

\section{Recorded hosts}

Pinaceae: Picea abies*, glauca, pungens, sitchensis.

\section{Distribution}

D F S. 
ALBRECHT A.C., Identification guide to Nordic aphids on conifers

A10 (A9) Overwintering larvae: Dorsal sclerites with groups of similar-sized ring-like wax pores. A1. gallicola with basal articulation of ant. IV and V narrower Adelges (Gilletteella Börner, 1930 and Sacciphantes Curtis, 1844)

Subgenus Gilletteella Börner, 1930

Adelges (Gilletteella) cooleyi (Gillette, 1907)

Figs 15-16

\section{Diagnosis}

Fund. juv: dorsal sclerites with groups of 8-30 ring-like wax pores. Al. gallicola: $1.7-2.5 \mathrm{~mm}$, wax glands on head and prothorax of al. gallicola with large distinct facets; rhinaria on ant. III-V transverse, slit-like. Gall 25-80 mm, spindle-shaped, needles from widened bases strongly protruding. Chambers separate with green, pink or red openings. Holocyclic and dioecious, alternating between Picea and Pseudotsuga (p. 28).

\section{Recorded primary hosts}

Pinaceae: Picea sitchensis (usually), engelmannii, glauca, jezoensis, mariana, pungens.

\section{Distribution}

D F N S.

Subgenus Sacciphantes Curtis, 1844

Adelges (Sacciphantes) abietis (Linnaeus, 1758)

Figs 17-21

\section{Diagnosis}

First instar pseudofundatrix about $0.5 \mathrm{~mm}$, greyish brown; prothoracic pleurospinal sclerites longer than broad; dorsal sclerites with groups of 2-7 ring-like wax pores. Second instar nymphs in winter about 0.6-0.8 mm. Wax filaments of overwintering nymphs numerous, long, glossy grey, stiff, curled in all directions. Adult pseudofundatrix greyish or yellowish brown, exuding abundant fine, white wax wool. Al. gallicola $1.8-2.3 \mathrm{~mm}$, brown to brownish black with thin greyish wax dusting and transverse white wax markings; abdomen yellowish brown; wax wool production fairly weak. Wax glands on head and prothorax absent or indistinct, without clear facets; rhinaria on antennal segments III-V oval to circular. Eggs brownish yellow. Anholocyclic on Picea, occurring in many habitats, usually 1-2 m above ground. In open positions such as forest margins, clearings and solitary trees, the scale margins of the galls become bright red or purple, whereas galls in shaded positions are less strikingly coloured.

The pseudofundatrices hibernate at bud bases and in bud axils, in contrast to $A$. tardus which prefers the surface of the buds (my own records from Finland). Second instar pseudofundatrices can be found from late autumn onwards, but first instar nymphs may occur as late as in February (according to my own records from S. Finland). Galls $15-35 \mathrm{~mm}$, usually gregarious, pineapple-shaped with needle-bearing shoot apically, bright green with purple, red, pink or brownish (rarely green) scale margins. Scales pubescent, particularly along margins. They often occur along with galls from preceding years and with galls of Adelges tardus; opening in August-early September. Gall chambers completely closed: no nymphs occur on outer surface.

\section{Recorded hosts}

Pinaceae: Picea abies*, engelmannii, glauca, jezoensis, orientalis, pungens, sitchensis, spp. 


\section{Distribution}

D F N S.

Adelges (Sacciphantes) viridis (Ratzeburg, 1843)

Figs 22-23

\section{Diagnosis}

Overwintering first instar fundatrix dark green; dorsal sclerites with groups of 2-7 ring-like wax pores; prothoracic pleurospinal sclerites almost square.

Al. gallicola about $2 \mathrm{~mm}$, dark olive to blackish with thin greyish wax dusting and transverse white wax markings; abdomen greenish or olive brown; wax wool production weak. Wax glands on head and prothorax absent or indistinct, without clear facets; rhinaria on ant. III-V oval to circular. Eggs green. Galls solitary, otherwise as in A. abietis. Holocyclic and dioecious, alternating between Picea and Larix.

\section{Recorded primary hosts}

Pinaceae: Picea abies*, engelmannii, glauca*, koraiensis, omorika, orientalis, pungens, sitchensis, spp.

\section{Distribution}

F.

A11 (A4) Siphunculi present; adults without sclerotised ovipositor. Apterae rarely less than $1.2 \mathrm{~mm}$; antennae at least 4-segmented Aphididae Latreille, 1802 A12

Superfamily Aphidoidea Latreille, 1802

Family Aphididae Latreille, 1802

A12 (A13 A14) Siphunculi elongate, tubular Elatobium Mordvilko, 1914, Aulacorthum Mordvilko, 1914, Neomyzus van der Goot, 1915 and Aphis Linnaeus, 1758

Subfamily Aphidinae Latreille, 1802

Tribe Macrosiphini Wilson, 1910

Genus Elatobium Mordvilko, 1914

Elatobium abietinum (Walker, 1849)

Fig. 24

\section{Diagnosis}

Aptera 1-2 mm, pale green to yellowish, without wax wool. Holocyclic, monoecious. In small groups on needles of Picea (rarely other genera), causing discoloration and loss of older needles. Not attended by ants.

\section{Recorded coniferous hosts}

Pinaceae: Picea abies*, engelmanni, glauca, glehnii, jezoensis, mariana, omorika, orientalis, pungens*, sitchensis, tianschanica; Abies cephalonica, homolepis, lasiocarpa, nordmanniana, spp.; Pseudotsuga menziesii; Pinus sibirica. 


\section{Distribution \\ D F Fa I N S.}

Genus Aulacorthum Mordvilko, 1914

Subgenus Aulacorthum Mordvilko, 1914

Aulacorthum (Aulacorthum) solani (Kaltenbach, 1843)

Fig. 25

\section{Diagnosis}

Aptera 1.2-2.6 mm, green with dark green spots in front of siphunculi. Polyphagous, very rarely on conifers. Not ant-attended.

\section{Recorded coniferous hosts}

Pinaceae: Picea abies, sitchensis.

\section{Distribution}

D F I N S.

Genus Neomyzus van der Goot, 1915

Neomyzus circumflexus (Buckton, 1876)

\section{Diagnosis}

Whitish, yellow or green; resembling Aulacorthum solani, but abdomen with brown or black markings, usually including a horseshoe-shaped patch. Polyphagous, very rarely on conifers. Not ant-attended.

\section{Recorded coniferous hosts}

Pinaceae: Picea sitchensis.

\section{Distribution}

D F N S.

Tribe Aphidini Latreille, 1802

Subtribe Aphidina Latreille, 1802

Genus Aphis Linnaeus, 1758

Subgenus Aphis Linnaeus, 1758

Aphis (Aphis) fabae Scopoli, 1763

Fig. 26

\section{Diagnosis}

Aptera 1.5-3 mm, dull black; legs and antennae partly white; abdomen sometimes with white wax markings; usually ant-attended. Polyphagous species (or group of species). Holocyclic and dioecious, primary hosts are Euonymus (Celastraceae), Philadelphus (Hydrangeaceae) and Viburnum (Adoxaceae). Numerous plants, mostly herbs, serve as secondary hosts. Only occasionally on conifers. Often attended by ants, but none recorded from conifers. 


\title{
Recorded coniferous hosts
}

Pinaceae: Picea sitchensis; Larix decidua, kaempferi; Tsuga heterophylla; Pinus contorta.

\section{Distribution}

D S N F I.

A13 (A12 A14) Siphunculi present as pores not raised from body surface ...Mindarus Koch, 1857

Subfamily Mindarinae Tullgren, 1909

Genus Mindarus Koch, 1857

Mindarus obliquus (Cholodkovsky, 1896)

Fig. 27

\section{Diagnosis}

Aptera 1.0-1.9 mm, pale yellowish green, with wax dusting, fine wax wool and thick, irregularly bent wax filaments yellowish green, very similar to M. abietis on Abies.

Three generations per year: fundatrix, al. and apterous progeny of the fundatrix and dwarfish sexuales. Holocyclic, monoecious on Picea. Colonies between needles of young shoots, not causing deformation. Sometimes attended by ants.

\section{Recorded hosts}

Pinaceae Picea engelmannii, glauca*, mariana, omorika, sitchensis, spp. (but not abies).

\section{Recorded attendant ants}

Formicinae: Lasius niger*.

\section{Distribution}

D F N S.

A14 (A12 A13) Siphunculi present as large pores elevated on obtuse cones Cinara Curtis, 1835

\author{
Subfamily Lachninae Herrich-Schaeffer, 1854 \\ Tribe Eulachnini Baker, 1920 \\ Genus Cinara Curtis, 1835 \\ Subgenus Cinara Curtis, 1835
}

Cinara (Cinara) piceae (Panzer, 1801)

Figs 28-29

\section{Diagnosis}

Aptera 4.5-7 mm, black, semi-matt with glossy sclerites, legs and antennae partly brownish red. Holocyclic, monoecious on Picea. Spruce and mixed forests. In dense colonies on stem and underside of branches, often high up in the trees, particularly on twigs with developing cones. Often also on young solitary spruces. Usually ant-attended. Moves restlessly around when disturbed and often falls to the ground where it may move to exposed roots. 


\section{Recorded hosts}

Pinaceae: Picea abies*, glauca, omorika, orientalis, pungens, sitchensis, tianschanica, spp. (Due to the agility of $C$. piceae and the similarity between $C$. piceae and $C$. curvipes, I suggest that records from Abies and other genera should be confirmed.)

\section{Recorded attendant ants}

Formicinae: Camponotus herculeanus*; Formica polyctena*, truncorum*; Lasius niger*.

\section{Distribution}

D F N S.

Cinara (Cinara) pilicornis (Hartig, 1841)

Figs $30-31$

\section{Diagnosis}

Aptera 2.1-4.7 mm, light red-brown, orange brown, olive or grey-green; abdominal dorsum usually with a conspicuous wax pattern The thickness of the wax cover varies considerably and wears easily off. Siphuncular cones small, brown or greenish black. Legs and antennae pale. Second segment of hind tarsus long and conspicuously curved. Hairs very long; on antennal segment 3, 2-3 times the diameter of the segment or even more. Alatae with abundant wax wool. Holocyclic, monoecious on Picea, also on Tsuga. Found in forest margins, clearings, often on solitary trees. Colonies on undersides of previous year's twigs, during summer colonising current year's shoots. Often ant-attended.

\section{Recorded hosts}

Pinaceae: Picea abies*, engelmannii, glauca*, omorika*, orientalis, pungens, sitchensis, tianschanica, spp.; Tsuga heterophylla?

\section{Recorded attendant ants}

Formicinae: Formica aquilonia*, fusca*, truncorum*; Lasius niger*. Myrmicinae: Myrmica rubra*.

\section{Distribution}

D F I N S.

\section{Cinara (Cinara) costata (Zetterstedt, 1828)}

Figs 32-33

\section{Diagnosis}

Apterae 2.5-3.5 mm, light brownish with olive green blackish pleural longitudinal bands forming a bottle-shaped pattern, and transverse bronzy stripes posteriorly; the dark parts with a characteristic metallic sheen. The wax cover is often heavy and obscures the underlying pattern, at least interrupting the longitudinal bands. Sometimes whole colonies may be covered in wax. Holocyclic, monoecious on Picea. Found in margins and clearings of spruce or mixed forests. In small, dense, wax-covered colonies on lignified twigs, usually on lower branches of tall trees. Normally not attended by ants. 


\section{Recorded hosts}

Pinaceae: Picea abies*, engelmannii, glauca*, omorika*, orientalis, pungens ${ }^{*}$, sitchensis, tianschanica, spp.

\section{Distribution}

D F N S.

Cinara (Cinara) pruinosa (Hartig, 1841)

Figs $34-37$

\section{Diagnosis}

Apterae 3-5.0 mm, olive or brown with dark green to black marking bands interrupted by transverse streaks and patches of wax dust. Often with metallic sheen. Adults with large black siphuncular cones. Holocyclic, monoecious on Picea. Colonies on branch undersides, shoots and trunks, later in summer also on roots. Usually ant-attended.

\section{Recorded hosts}

Pinaceae: Picea abies*, glauca, orientalis, pungens, sitchensis, tianschanica, spp.

\section{Recorded attendant ants}

Formicinae: Formica aquilonia*, lugubris*, polyctena*, truncorum*; Lasius niger*. Myrmicinae: Myrmica rubra*, ruginodis*.

\section{Distribution}

D F N S.

Cinara (Cinara) piceicola (Cholodkovsky, 1896)

Figs 38-39

\section{Diagnosis}

Apterae 2-4.2 mm, grey-green, olive or pale brown with darker head and thorax and two longitudinal darker stripes. Siphuncular cones small, inconspicuous. Holocyclic, monoecious on Picea. Colonies on lignified shoots and branches, on tall as well as small spruces, in summer also on subterraneous parts. Often at ground level on dwarfish spruces on sandy ground at roadsides and ditch margins. Usually antattended.

\section{Recorded hosts}

Pinaceae: Picea abies*, glauca, orientalis, pungens, sitchensis, spp.

\section{Recorded attendant ants}

Formicinae: Formica aquilonia*, lugubris*, pratensis*, sanguinea*, truncorum*; Lasius fuliginosus*, niger*. Myrmicinae: Myrmica rubra*.

\section{Distribution}

D F N S. 


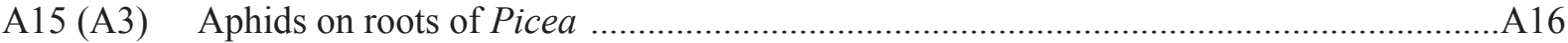

A16 (A17) Apterae 2-6 mm, with brown, green or blackish colours; siphunculi present as pores elevated on cones. On thick roots, usually ant-attended

Cinara Curtis, 1835 go to entry point A14 (p. 14)

A17 (A16) Apterae 0.5-2 mm, whitish, pale green or yellowish, exuding abundant white wax wool; siphunculi absent. On thin mycorrhizal root ends, not attended by ants

Eriosomatinae Kirkaldy, 1905

\section{Subfamily Eriosomatinae Kirkaldy, 1905}

For key and diagnoses, see Albrecht (2015: 8, key A).

Tribe Pemphigini Herrich-Schaeffer, 1854

Genus Pachypappella Baker, 1920

Pachypappella lactea (Tullgren, 1909)

\section{Diagnosis}

Holocyclic, dioecious, alternating between Populus tremula (Salicaceae) and Picea, and maintaining a continuous anholocyclic population on Picea.

\section{Recorded secondary hosts}

Pinaceae: Picea abies*, sitchensis.

\section{Distribution}

D F S.

Genus Pachypappa Koch, 1856

Pachypappa populi (Linnaeus, 1758)

\section{Diagnosis}

Holocyclic, dioecious, alternating between Populus tremula and Picea. Anholocyclic hibernation on roots of Picea is common.

\section{Recorded secondary hosts}

Pinaceae: Picea abies*.

\section{Distribution}

F N S.

Pachypappa tremulae (Linnaeus, 1761)

\section{Diagnosis}

Holocyclic, dioecious, alternating between Populus tremula (Salicaceae) and Picea. Anholocyclic hibernation on roots of Picea is common.

\section{Recorded secondary hosts}

Pinaceae: Picea abies*, orientalis, sitchensis.

\section{Distribution}

D F N S. 


\section{Pachypappa vesicalis Koch, 1856}

\section{Diagnosis}

Holocyclic, dioecious, alternating between Populus alba (Salicaceae) and Pinaceae: Picea and Pseudotsuga.

\section{Recorded secondary hosts}

Pinaceae: Picea abies*, glauca, sitchensis; Pseudotsuga menziesii.

\section{Distribution}

F S.

Genus Prociphilus Tullgren, 1925

Subgenus Stagona Koch, 1857

Prociphilus (Stagona) xylostei (De Geer, 1773)

\section{Diagnosis}

Holocyclic and dioecious, alternating between Lonicera (Caprifoliaceae) and Picea. An anholocyclic population on Picea roots is upheld throughout the year. Not ant-attended.

\section{Recorded secondary hosts}

Pinaceae: Picea abies*, glauca, sitchensis; Pseudotsuga menziesii. According to Holman (2009) also on Abies.

\section{Distribution}

D F N S.

\section{Key B. Aphids on fir (Abies)}

\section{Synopsis}

B1 (B8) Body, at least in adults, covered with white wax wool, filaments or ribbons; siphunculi absent

B2 (B3) On roots, forming colonies embedded in wax wool .............Prociphilus Koch, 1857 p. 19

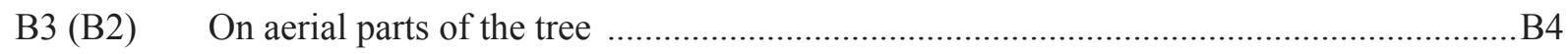

B4 (B7) Antennae at most 3-segmented. Scattered on bark or needles ………………................. B5

B5 (B6) On needles ...................................................Adelges (Aphrastasia) Börner, 1909 p. 20

B6 (B5) On bark of stems, branches and twigs ...................Adelges (Dreyfusia) Börner, 1908 p. 20

B7 (B4) Antennae 6-segmented. In colonies between needles of young shoots

..Mindarus Koch, 1857 p. 21

B8 (B1) Wax, if present, in the form of dusting, siphunculi present . B9

B9 (B10) Siphunculi present as pores raised on cones Cinara Curtis, 1835 p. 22

B10 (B9) Siphunculi tubular Elatobium Mordvilko, 1914 p. 23 
ALBRECHT A.C., Identification guide to Nordic aphids on conifers

B1 (B8) Body, at least in adults, covered with white wax wool, filaments or ribbons; siphunculi absent B2

B2 (B3) On roots, forming colonies embedded in wax wool Prociphilus Koch, 1857

Superfamily Aphidoidea Latreille, 1802

Family Aphididae Latreille, 1802

Subfamily Eriosomatinae Kirkaldy, 1905

Tribe Pemphigini Herrich-Schaeffer, 1854

Genus Prociphilus Koch, 1857

Subgenus Prociphilus Koch, 1857

Prociphilus (Prociphilus) bumeliae (Schrank, 1801)

Fig. 40

\section{Diagnosis}

Apterae about $2.9 \mathrm{~mm}$, covered with wax wool. Two pairs of wax gland plates on head, of which the posterior ones better developed than the anterior. A narrow pale subapical zone on RIV+V present. Diagnosis based on Heie (2004). Holocyclic and dioecious, alternating between ash Fraxinus excelsior (sometimes other Oleaceae such as Ligustrum and Syringa) and Abies, where it feeds in wax-covered colonies on the roots. Not ant-attended.

\section{Recorded secondary hosts}

Pinaceae: Abies alba, veitchii, spp.

\section{Distribution}

D F S.

Prociphilus (Prociphilus) fraxini (Fabricius, 1777)

\section{Diagnosis}

Apterae 1.8-2.7 mm, pale, wax-covered. Very similar to P. bumeliae, but with posterior wax gland plates on head weakly developed, sometimes absent. Diagnosis based on Heie (2004). Holocyclic and dioecious, alternating between Fraxinus excelsior (Oleaceae) and roots of Abies, where wax-covered colonies are formed. Not ant-attended.

\section{Recorded secondary hosts}

Pinaceae: Abies alba, balsamea, nordmanniana, sibirica, spp.

\section{Distribution}

D N S.

\section{Remarks}

Holman (2009) also lists Prociphilus (Stagona) xylostei from Abies roots. See under Picea (entry A17, p. 17). 


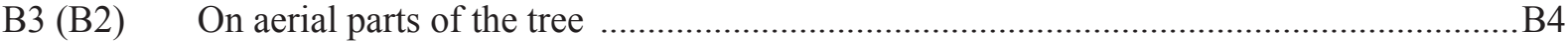

B4 (B7) Antennae at most 3-segmented. Scattered on bark or needles ……................................. B5

B5 (B6) On needles .............................................................Adelges (Aphrastasia) Börner, 1909

Superfamily Adelgoidea Annand, 1928

Family Adelgidae Annand, 1928

Genus Adelges Vallot, 1836

Subgenus Aphrastia Börner, 1909

Adelges (Aphrastasia) pectinatae (Cholodkovsky, 1888)

Figs 41-44

\section{Diagnosis}

Neosistens $0.3-0.4 \mathrm{~mm}$, shiny black with a double spinal crest and a marginal rim of more or less curly wax ribbons, and a posterior tuft of wax wool. Older sistentes and progredientes dark grey, embedded in a tuft of thick, stiff, 'half-pipe' shaped wax filaments. Spinal and pleural sclerites fused on all segments, and spinopleural sclerites also fused to marginal sclerites of abdominal tergites 6-8. Older sistentes and progredientes dark grey to purplish grey, richly exuding thick, curly wax filaments. Holocyclic and dioecious, alternating between spruce (Picea, p. 8) and fir (Abies). Predominantly in parks and plantations, scattered on needles, both upper- and undersides, often in great number. Not ant-attended. Apparently predominantly anholocyclic on Abies. Not ant-attended.

\section{Recorded secondary hosts}

Pinaceae: Abies alba*, amabilis*, balsamea*, concolor*, fraseri*, grandis*, koreana*, lasiocarpa*, nordmanniana, procera, sibirica*, veichtii, spp.

\section{Distribution}

F N S.

B6 (B5) On bark of stems, branches and twigs ............................Adelges (Dreyfusia) Börner, 1908

Subgenus Dreyfusia Börner, 1908

Adelges (Dreyfusia) nordmannianae (Eckstein, 1890)

Figs 45-46

\section{Diagnosis}

Neosistens rather similar to those of $A$. pectinatae (see above), but wax ribbons thinner and more strongly curled, and spinal and pleural sclerites on mesothorax, metathorax and abdominal tergites 1-5 separate (although sometimes touching). Older sistentes and progredientes dark grey, embedded in fine, curly wax wool. Wax pore plates on inner margins of meso- and metathoracic spinal sclerites containing numerous small, mostly rounded, pits arranged in 2-4 areas of rather rounded shape, the most central area containing 7-12 pits. Found on bark, usually on young branches and twigs, single specimens occasionally on needles (my own record). Not ant-attended.

\section{Recorded secondary hosts}

Pinaceae: Abies alba*, balsamea, cephalonica, concolor, grandis, nordmanniana, procera, sibirica, veitchii, spp. 


\section{Distribution}

D N S.

Adelges (Dreyfusia) merkeri (Eichhorn, 1957)

\section{Diagnosis}

Similar to A. nordmannianae. Neosistens: wax pore plates on inner margins of meso- and metathoracic spinal sclerites divided into 3-4 rounded areas, each with 3-8 pits. Total number of pits on the 10 central areas 26-63 (usually more than 40) (Blackman \& Eastop 1994, 2014). Holocyclic and dioecious, alternating between Picea orientalis (p. 9) and Abies alba. Scattered on trunk and older branches, on all parts of seedlings. Not ant-attended.

\section{Recorded secondary hosts}

Pinaceae: Abies alba. Holman (2009) also lists A. nordmanniana.

\section{Distribution}

S.

Adelges (Dreyfusia) piceae (Ratzeburg, 1843)

Fig. 47

\section{Diagnosis}

Similar to A. merkeri but neosistens with central areas of wax pore plates on meso- and metathoracic spinal sclerites often more-or-less triangular, each with 3-6 pits. Total number of pits on 10 central areas $18-59$, usually less than 40 . The characters distinguishing $A$. piceae and $A$. merkeri are variable, and the identification requires several specimens (Blackman \& Eastop 2006; Heie 2004). Monoecious, anholocyclic on Abies. Found on trunk and branches. Not ant-attended.

\section{Recorded hosts}

Pinaceae: Abies alba, amabilis, balsamea, concolor, lasiocarpa, nordmanniana, sibirica, spp.

\section{Distribution}

D.

B7 (B4) Antennae 6-segmented. In colonies between needles of young shoots.

Mindarus Koch, 1857

Superfamily Aphidoidea Latreille, 1802

Family Aphididae Latreille, 1802

Subfamily Mindarinae Tullgren, 1909

Genus Mindarus Koch, 1857

Mindarus abietinus (Koch, 1857)

Figs 48-49.

\section{Diagnosis}

Aptera 1.7-2.0 mm, pale green, with wax dusting, fine wax wool and thick, irregularly bent wax filaments. Alata pale green with transverse sclerites on abd. dorsum, body and appendages with short, 
fine wax wool. Holocyclic, monoecious on Abies. In colonies between needles on young shoots. Only 3 generations per year. Sexuales dwarfish, in June-July. Not ant-attended.

\title{
Recorded hosts
}

Pinaceae: Abies alba, balsamea, cephalonica, concolor, grandis, homolepis, lasiocarpa, nordmanniana, pindrow, procera, sibirica*; Pseudotsuga menziesii.

\section{Distribution}

D F N S.

B8 (B1) Wax, if present, in the form of dusting, siphunculi present B9

B9 (B10) Siphunculi present as pores raised on cones Cinara Curtis, 1835

\author{
Subfamily Lachninae Herrich-Schaeffer, 1854 \\ Tribe Eulachnini Baker, 1920 \\ Genus Cinara Curtis, 1835 \\ Subgenus Cinara Curtis, 1835
}

Cinara (Cinara) confinis Koch, 1856

Figs $50-52$

\section{Diagnosis}

Siphunculi short and raised on cones. Aptera 3.8-7.5 mm, dark brown to brownish black with a bronzy sheen, legs predominantly black. Venter and segment borders thinly wax-dusted. Siphunculi short, raised on cones. Holocyclic and monoecious on Abies. Forming colonies, often dense, on young parts of trunks and underside of branches. Sometimes ant-attended.

\section{Recorded hosts}

Pinaceae: Abies alba, balsamea, cephalonica, concolor, grandis, holophylla*, lasiocarpa, nordmanniana, pindrow, procera, sibirica*, spp.

\section{Recorded attendant ants}

Formicinae: Formica rufa*.

\section{Distribution}

D F N S.

Cinara (Cinara) curvipes (Patch, 1912)

Fig. 53

\section{Diagnosis}

Aptera 3.4-5.5 mm subshining black, legs orange to red-brown, tarsi and hind tibiae partly black. Juveniles and adults in summer with a thin but rather dense greyish wax cover. Siphunculi short, raised on cones. In appearance similar to C. piceae (see under Picea, entry A14, p. 14). Holocyclic and monoecious on Abies (and Cedrus). On trunk and branches. Sometimes attended by ants.

\section{Recorded hosts}

Pinaceae: Abies balsamea, concolor, koreana, lasiocarpa, spp.; Cedrus deodara. 


\section{Recorded attendant ants}

Formica rufa (group?).

\section{Distribution}

Introduced from North America. Not yet in the Nordic countries, but widespread in Central and Western Europe.

\section{Cinara (Cinara) pectinatae Nördlinger, 1880}

Figs $54-55$

\section{Diagnosis}

Aptera $2.8-5 \mathrm{~mm}$, bright green. Abdomen dorsally with diffuse whitish longitudinal bands, juveniles often with an additional whitish spinal streak; legs infuscate; eyes red. Underside and segment borders on thorax thinly wax-dusted. Holocyclic, monoecious on Abies. In small groups or colonies on young trunks and underside of lignified branches, sometimes ant-attended.

\section{Recorded attendant ants}

Formicinae: Formica polyctena*.

\section{Recorded hosts}

Pinaceae: Abies alba* (usually), balsamea, grandis, nordmanniana, sibirica spp.

\section{Distribution}

D N S.

B10 (B9) Siphunculi tubular Elatobium Mordvilko, 1914

Subfamily Aphidinae Latreille, 1802

Tribe Macrosiphini Wilson, 1910

Genus Elatobium Mordvilko, 1914

Elatobium abietinum Walker, 1849

\section{Diagnosis}

Aptera 1-2 mm, green to yellowish, without wax wool. On needles, rarely on Abies. See under Picea (entry A12, p. 12).

\section{Key C. Aphids on larch (Larix)}

\section{Synopsis}

C1 (C2) Siphunculi absent. Apterae: antennae with at most 3 segments. Body less than $2.5 \mathrm{~mm}$, often covered with white wax wool or filaments Adelges Vallot, 1836 p. 24

C2 (C1) Siphunculi present. Antennae with at least 4 segments. Body usually larger than $2.5 \mathrm{~mm}$. Wax, if present, in the form of dusting or well-defined pattern

C3 (C4) Siphunculi present as pores raised on cones Cinara Curtis, 1835 p. 26

C4 (C3) Siphunculi elongate, tubular Aphis Linnaeus, 1758 p. 27 
C1 (C2) Siphunculi absent. Apterae: antennae with at most 3 segments. Body less than $2.5 \mathrm{~mm}$, often covered with white wax wool or filaments Adelges Vallot, 1836

Superfamily Adelgoidea Annand, 1928

Family Adelgidae Annand, 1928

Genus Adelges Vallot, 1836

Subgenus Sacciphantes Curtis, 1844

Adelges (Sacciphantes) viridis (Ratzeburg, 1843)

Figs $56-57$

\section{Diagnosis}

Neosistens: $0.5-0.8 \mathrm{~mm}$, dull green with small, roundish wax gland plates on head, thorax and abdomen. Wax glands with double-walled facets secreting hollow tubes of wax. Sexuparae on Larix in spring pale greenish with sparse wax secretion. Aptera in summer covered with fine wax wool. Alatae 1.3-1.7 mm. Holocyclic and dioecious, alternating between Picea and Larix. On Larix overwintering at bud bases or in bark crevices on twigs and branches. In spring and summer on needles. Not ant-attended.

\section{Recorded secondary hosts}

Pinaceae: L. decidua*, decidua x kaempferi, kaempferi, sibirica*, spp.

\section{Distribution}

F.

Subgenus Adelges Vallot, 1836

Adelges (Adelges) laricis Vallot, 1836

Figs 58-61

\section{Diagnosis}

Neosistens: About $0.3 \mathrm{~mm}$, brownish black, without wax secretion. Dorsal plates of head and thorax fused. Dorsum heavily sclerotised and somewhat wrinkled. Adult aptera oval or sub-circular, blackish or dark grey, about $1 \mathrm{~mm}$., usually with wax wool. Alata $1.0-1.5 \mathrm{~mm}$, head with anterior and posterior wax gland areas, if present, usually separate. Al. gallicola (migrating from Picea) with poorly developed wax secretion. Holocyclic and dioecious, alternating between Picea (p. 9) and Larix. On Larix overwintering at bud bases or in bark crevices on twigs and branches. In spring and summer on needles. Not antattended.

\section{Recorded secondary hosts}

Pinaceae: Larix decidua*, decidua x kaempferi, gmelinii, kaempferi*, laricina*, lyallii*, sibirica*, spp.

\section{Distribution}

D F N S. 


\section{Diagnosis}

In most respects similar to A. laricis. The al. gallicolae secrete abundant wax. Holocyclic and dioecious, alternating between Picea (p. 10) and Larix.

\section{Recorded secondary hosts}

Pinaceae: Larix sibirica.

\section{Distribution}

Europe. Not recorded in the Nordic countries.

Subgenus Cholodkovskya Börner, 1909

Adelges (Cholodkovskya) viridanus (Cholodkovsky, 1896)

Fig. 62

\section{Diagnosis}

Neosistens: $0.6-0.7 \mathrm{~mm}$, yellow or yellow-green; dorsal plates of head and thorax separate; head with 5 pairs of small wax gland fields; dorsum membranous. Adult sistens pyriform, yellowish green, with wax wool, 1.8-2.5 mm. Eggs yellow. Alatae 2.0-2.6 mm, greenish grey, head with anterior and posterior wax gland areas united. Anholocyclic on Larix. On needles in summer, overwintering in bark crevices. Not ant-attended.

\section{Recorded hosts}

Pinaceae: Larix decidua x kaempferi, gmelini, kaempferi, sibirica, spp.

\section{Distribution}

S.

Adelges (Cholodkovskya) viridulus (Cholodkovsky, 1911)

\section{Diagnosis}

Very similar to $A$. viridanus. Neosistens with fewer wax pores on posterior abdominal segments than A. viridanus (Blackman \& Eastop 2016). Anholocyclic on Larix.

\section{Recorded hosts}

Pinaceae: Larix sibirica.

\section{Distribution}

Northern Russia. 
C2 (C1) Siphunculi present. Antennae with at least 4 segments. Body usually larger than $2.5 \mathrm{~mm}$. Wax, if present, in the form of dusting or well-defined pattern

C3 (C4) Siphunculi present as pores raised on cones

Cinara Curtis, 1835

Superfamily Aphidoidea Latreille, 1802

Family Aphididae Latreille, 1802

Subfamily Lachninae Herrich-Schaeffer, 1854

Tribe Eulachnini Baker, 1920

Genus Cinara Curtis, 1835

Subgenus Cinara Curtis, 1835

Cinara (Cinara) laricis (Hartig, 1839)

Figs $63-65$

\section{Diagnosis}

Aptera 3-5 mm, subshining brown with numerous small black hair-bearing sclerites and usually with a greyish wax bloom pattern rendering a conspicuous chequered appearance. Holocyclic, monoecious on Larix. Found in forest margins, meadows, parks, plantations etc. In small colonies or scattered along 1-3 year-old twigs of tall trees, on young shoots and cones, and trunks of young trees. Sometimes antattended.

\section{Recorded hosts}

Pinaceae: Larix decidua*, decidua x kaempferi, gmelinii, leptolepis, sibirica*.

\section{Recorded attendant ants}

Formicinae: Formica rufa*; Lasius niger*.

\section{Distribution}

D F S B.

Cinara (Cinara) cuneomaculata (del Guercio, 1909)

Figs 66-68

\section{Diagnosis}

Aptera 2.3-4.5 mm, olive brown to dark purplish brown, subshining with a thin greyish wax bloom ventrally and also as a transverse pattern on dorsum, much like the pattern of $C$. laricis. Small, hairbearing sclerites absent. Holocyclic, monoecious on Larix. Found in forest margins, meadowland etc. In small colonies or scattered along young twigs (usually 0-2 years old). Often ant-attended.

\section{Recorded hosts}

Pinaceae: Larix decidua*, kaempferi*, laricina, sibirica*, spp.

\section{Recorded attendant ants}

Formicinae: Formica rufa*. 


\title{
Distribution
}

D F S.

Cinara (Cinara) kochiana (Börner, 1939)

Fig. 69

\section{Diagnosis}

Aptera 4-6 mm, brown to greyish-brown, slightly wax-powdered on dorsum. Monoecious, holocyclic on Larix. In colonies in bark crevices on lower part of trunk or bases of older branches, also on more or less exposed roots. Usually ant-attended. Diagnosis based on Heie (2004).

\section{Recorded hosts}

Pinaceae: Larix decidua, gmelinii, kaempferi, sibirica.

\section{Recorded attendant ants}

Formicinae: Formica lugubris.

\section{Distribution}

D S.

C4 (C3) Siphunculi elongate, tubular Aphis Linnaeus, 1758

\author{
Subfamily Aphidinae Latreille, 1802 \\ Tribe Aphidini Latreille, 1802 \\ Subtribe Aphidina Latreille, 1802 \\ Genus Aphis Linnaeus, 1758 \\ Subgenus Aphis Linnaeus, 1758 \\ Aphis (Aphis) fabae Scopoli, 1763
}

\section{Diagnosis}

Aptera 1.5-3 mm, dull black; legs and antennae partly white; abdomen sometimes with white wax markings. See under Picea (entry A12, p. 12).

\section{Key D. Aphids on hemlock and Douglas fir (Tsuga and Pseudotsuga)}

\section{Synopsis}

D1 (D4) On young shoots or needles ...... .D2

D2 (D3) Siphunculi absent Adelges Vallot, 1836 and Mindarus Koch, 1857 p. 28

D3 (D2) Siphunculi present, tubular. Aphis Linnaeus, 1758 p. 29

D4 (D1) On thin mycorrhizal roots Eriosomatinae Kirkaldy, 1905 p.29 
D1 (D4) On young shoots or needles D2

D2 (D3) Siphunculi absent Adelges Vallot, 1836 and Mindarus Koch, 1857

Superfamily Adelgoidea Annand, 1928

Family Adelgidae Annand, 1928

Genus Adelges Vallot, 1836

Subgenus Gilletteella Börner, 1930

Adelges (Gilletteella) cooleyi (Gillette, 1907)

Figs 70-71

Diagnosis (see also p. 11)

Apterae brownish black, covered with wax wool. Neosistens $0.3-0.4 \mathrm{~mm}$, black. Dorsum with rounded wax gland plates exuding sparsely white wax. Marginally with glands exuding broad and fairly short wax ribbons, and posteriorly with a tuft of long narrow ribbons. Al. sexuparae 1.2-1.7 mm; abdomen with normal spinal and pleural wax gland plates on all segments; rhinaria at most reaching half-way around the segments. Holocyclic and dioecious, alternating between Picea (p. 11) and Pseudotsuga.

\title{
Recorded secondary hosts
}

Pinaceae: Pseudotsuga menziesii*.

\section{Distribution}

D F N S.

\author{
Superfamily Aphidoidea Latreille, 1802 \\ Family Aphididae Latreille, 1802 \\ Subfamily Mindarinae Tullgren, 1909 \\ Genus Mindarus Koch, 1857
}

Mindarus abietinus (Koch, 1857)

\section{Diagnosis}

Aptera 1.7-2.0 mm, pale green, with wax dusting, fine wax wool and thick, irregularly bent wax filaments. See under Abies (entry B7, p. 21).

Subfamily Aphidinae Latreille, 1802

Tribe Macrosiphini Wilson, 1910

Genus Elatobium Mordvilko, 1914

Elatobium abietinum (Walker, 1849)

\section{Diagnosis}

Aptera 1-2 mm, pale green to yellowish, without wax wool. See under Picea (entry A12, p. 12). 
ALBRECHT A.C., Identification guide to Nordic aphids on conifers

D3 (D2) Siphunculi present, tubular. Aphis Linnaeus, 1758

Tribe Aphidini Latreille, 1802

Genus Aphis Linnaeus, 1758

Subgenus Aphis Linnaeus, 1758

Aphis (Aphis) fabae Scopoli, 1763

\section{Diagnosis}

Aptera $1.5-3 \mathrm{~mm}$, dull black; legs and antennae partly white; abdomen sometimes with white wax markings. See under Picea (entry A12, p. 12).

D4 (D1) On thin mycorrhizal roots

Eriosomatinae Kirkaldy, 1905

Eriosomatinae Kirkaldy, 1905

If pinkish, see under Pinus (entry E13, p. 39), otherwise see under Picea (entry A17 p. 17). For key and diagnoses, see Albrecht (2015: 8, key A).

\section{Key E. Aphids on pine (Pinus, Pinaceae)}

\section{Synopsis}

E1 (E11) Aphids on aerial parts of Pinus

E2 (E3) Siphunculi absent; adults with sclerotised ovipositor. Apterae less than $1.2 \mathrm{~mm}$; antennae at most 3-segmented Pineus Shimer, 1869 p. 30

E3 (E2) Siphunculi present at least as pores; adults without sclerotised ovipositor. Apterae rarely less than $1.2 \mathrm{~mm}$; antennae at least 4 -segmented ........................................................

E4 (E10) Siphunculi present as pores ............................................................................. E5

E5 (E6 E7) Body slender, at least twice as long as wide, with or without wax bloom

Eulachnus del Guercio, 1909 p. 31

E6 (E5 E7) Body oval; dark greyish brown with abundant wax wool; apical segment of rostrum short, hardly twice as long as wide Schizolachnus Mordvilko, 1909 p. 33

E7 (E5 E6) Body oval, grey, brown or blackish, wax, if present, as a distinct pattern or thin wax dusting; apical segment of rostrum long and narrow, 3 times as long as wide or more.

Cinara Curtis, 1835 E8

E8 (E9) Segment 1 of hind tarsus short; its dorsal side about half the length of the ventral

Cinara (Cinara) Curtis, 1835 p. 34

E9 (E8) Hind tarsus 1 long; its dorsal side $2 / 3$ the length of the ventral or more

Cinara (Cinara) Curtis, 1835 p. 36

E10 (E4) Siphunculi elongate, tubular

Elatobium Mordvilko, 1914, Aphis Linnaeus, 1758 p. 39

E11 (E1) Aphids on roots of Pinus

E12

E12 (E13) Apterae 2-6 mm, with brown, grey or blackish colours; siphunculi present as pores elevated on cones. Usually on thick exposed roots

Cinara Curtis, 1835 p. 39 
E13 (E12) Apterae 0.5-2 mm, pale pinkish, exuding abundant white wax wool; siphunculi absent. On thin roots Prociphilus Tullgren, 1925 p. 39

E1 (E11) Aphids on aerial parts of Pinus E2

E2 (E3) Siphunculi absent; adults with sclerotised ovipositor. Apterae less than $1.2 \mathrm{~mm}$; antennae at most 3-segmented Pineus Shimer, 1869

Superfamily Adelgoidea Annand, 1928

Family Adelgidae Annand, 1928

Genus Pineus Shimer, 1869

Subgenus Pineus Shimer, 1869

Pineus (Pineus) pini (Macquart, 1819)

Figs $72-75$

\section{Diagnosis}

Apterae $0.8-1.1 \mathrm{~mm}$, broadly drop-shaped in outline, dark red-brown, embedded in dense, fine wax wool. Wax glands on anterior part of cephaloprothoracic shield (between the eyes) mainly comprising groups of 2-10 facets that are loosely grouped, round in outline. Alatae 1.0-1.2 mm, reddish black with woolly wax. P. pini prefers open habitats: pine forests, forest clearings and margins, meadows, roadsides etc. Anholocyclic on Pinus. One overwintering generation on thin trunks or twigs and two or more overlapping generations on young shoots. See also P. orientalis below.

\section{Recorded hosts}

Pinaceae: Pinus banksiana, mugo*, nigra, pinaster?, ponderosa, rigida, sylvestris*.

\section{Distribution}

D F I N S.

Pineus (Pineus) orientalis (Dreyfus, 1889)

Fig. 76

\section{Diagnosis}

Apterae indistinguishable from $P$. pini. Gallicolae similar to apterae of $P$. pini but larger $(1.5-2.3 \mathrm{~mm})$; marginal wax gland plates smaller, their facets smaller than the largest on head; anteriorly on abdomen the plates are as far or further apart than the width of the plates; the spinal plates on abdomen fused with the other plates on their segment. Sexupara indistinguishable from the alata of $P$. pini. Holocyclic, dioecious, alternating between Picea (see p. 7) and Pinus.

Scattered on twigs and shoots. Diagnosis mainly after Heie (2004).

\section{Recorded secondary hosts}

Pinaceae: Pinus mugo, sylvestris, spp.

\section{Distribution}

D F S. 
Pineus (Pineus) cembrae (Cholodkovsky, 1888)

Figs $77-80$

\title{
Diagnosis
}

Apterae $0.5-0.8 \mathrm{~mm}$, red-brown with blackish sclerites, covered in wax wool. Wax glands on cephaloprothoracic shield with closely packed, more or less polygonal facets; groups closest to ocelli usually with more than 40 facets. Holocyclic, dioecious, alternating between Picea (see p. 7) and Pinus. At least in Finland also anholocyclic on Pinus. Parks, forests, street plantations. Scattered on young shoots, twigs and cones, overwintering in bark crevices and under scales.

\section{Recorded secondary hosts}

Pinaceae: Pinus cembra*, koraiensis, pumila, sibirica*.

\section{Distribution}

F S.

Pineus (Pineus) strobi (Hartig, 1837)

Fig. 81

\section{Diagnosis}

Apterae $0.7-0.9 \mathrm{~mm}$, similar to P. cembrae. Wax glands on cephaloprothoracic shield between eyes usually with less than 30 facets per group. Anholocyclic on Pinus. Found on shaded parts of trunk and branches. Alatae (sexuparae) fly to Picea mariana and lay eggs, but the sexual phase is abortive. Diagnosis based on Heie (2004) and Blackman \& Eastop (1994).

\section{Recorded hosts}

Pinaceae: Pinus peuce, sibirica, strobus.

\section{Distribution}

D N S (North America, introduced to Europe).

E3 (E2) Siphunculi present at least as pores; adults without sclerotised ovipositor. Apterae rarely

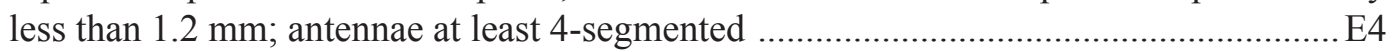

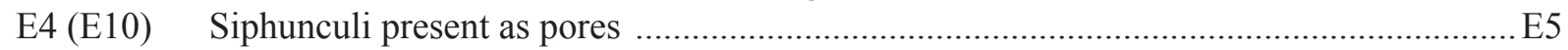

E5 (E6 E7) Body slender, at least twice as long as wide, with or without wax bloom ........................

\author{
Superfamily Aphidoidea Latreille, 1802 \\ Family Aphididae Latreille, 1802 \\ Subfamily Lachninae Herrich-Schaeffer, 1854 \\ Tribe Eulachnini Baker, 1920 \\ Genus Eulachnus del Guercio, 1909 \\ Eulachnus agilis (Kaltenbach, 1843)
}

Fig. 82

\section{Diagnosis}

Apterae 1.5-2.2 mm, elongate, green, thinly wax-powdered, with numerous dark green or brown hair-bearing sclerites. Dorsal hairs 1.5-2 times as long as the diameter of their sclerite. Holocyclic, 
monoecious on Pinus. Scattered on needles, rarely on twigs. E. agilis seems to prefer older trees and needles injured by Schizolachnus species. Not ant-attended.

\section{Recorded hosts}

Pinaceae: Pinus banksiana*, cembra, mugo*, nigra, sibirica, sylvestris*, spp.

\section{Distribution}

D F N S.

Eulachnus brevipilosus Börner, 1940

\section{Diagnosis}

Similar to $E$. agilis but dorsal hairs much shorter, about half the diameter of their sclerites. First segment of hind tarsus with 6 ventral hairs. Hairs on antennal segment III capitate. Holocyclic, monoecious on Pinus. Biology as in E. agilis. Diagnosis based on Heie (2004) and Blackman \& Eastop (1994).

\section{Recorded hosts}

Pinaceae: Pinus mugo, nigra, sylvestris*, spp.

\section{Distribution}

D F N S.

Eulachnus nigricola (Pasek, 1953)

\section{Diagnosis}

Similar to E. brevipilosus, but first segment of hind tarsus with 8 ventral hairs, and hairs on antennal segment III not capitate. Holocyclic, monoecious on Pinus nigra. Biology as in E. agilis. Diagnosis based on Heie (2004) and Blackman \& Eastop (1994).

\section{Recorded hosts}

Pinaceae: Pinus nigra, sylvestris.

\section{Distribution}

D.

Eulachnus cembrae Börner, 1950

\section{Diagnosis}

Apterae 2.2-3 mm, elongate, bright green or yellowish green, wax-powdered. Dorsal hairs on abdominal segments 1-6 not arising from sclerites. Holocyclic, monoecious on Pinus. Not ant-attended. Diagnosis based on Heie (2004) and Blackman \& Eastop (1994).

\section{Recorded hosts}

Pinaceae: Pinus cembra, peuce, strobus.

\section{Distribution}

Montane Europe. Not recorded in the Nordic countries. 
Fig. 83

\section{Diagnosis}

Apterae 2-2.8 mm, elongate, grey, brown, orange or olive, with grey, often coarse, wax dusting, often forming transverse fasciae. Body hairs long, dorsal hairs on abdomen more than twice the diameter of their sclerites. Holocyclic, monoecious on Pinus, at least in Finland clearly preferring P. mugo. Adults on twigs and branches. Juveniles in rows on needles, frequently causing yellowing. Not ant-attended.

\section{Recorded hosts}

Pinaceae: Pinus austriaca, banksiana, cembra, mugo*, nigra, peuce, sylvestris, spp.

\section{Distribution}

D F N S.

E6 (E5 E7) Body oval; dark greyish brown with abundant wax wool; apical segment of rostrum short, hardly twice as long as wide Schizolachnus Mordvilko, 1909

Genus Schizolachnus Mordvilko, 1909

Schizolachnus pineti (Fabricius, 1781)

Figs $84-85$

\section{Diagnosis}

Apterae $1.2-1.5 \mathrm{~mm}$, grey, grey-brown or brown, covered with greyish wax. The ultimate part of rostrum, the so-called segment $\mathrm{V}$, as long as its basal width. Hind tibia pale or dark, very densely hairy. Holocyclic, monoecious on many Pinus species, preferably sylvestris and mugo, forming dense rows along upper sides of last year's needles, on young as well as old trees. Not ant-attended.

\section{Recorded hosts}

Pinaceae: Pinus cembra, contorta, nigra, pinaster, pinea, banksiana*, mugo*, sylvestris*, spp.

\section{Distribution}

D F N S.

Schizolachnus obscurus Börner, 1940

Fig. 86

\section{Diagnosis}

Apterae 1.9-2.7 mm, brownish, covered with greyish white wax. The ultimate part of rostrum about $1.5 \times$ as long as it basal width. Hind tibia dark, less densely hairy than in S. pineti. Holocyclic, monoecious. Forming rows on needles of Pinus nigra (sometimes on other Pinus species). Not ant-attended. Diagnosis based on Heie (2004).

\section{Recorded hosts}

Pinaceae: Pinus nigra, rarely mugo, pinaster, pinea, sylvestris.

\section{Distribution}

D. 
E7 (E5 E6) Body oval, grey, brown or blackish, wax, if present, as a distinct pattern or thin wax dusting; apical segment of rostrum long and narrow, 3 times as long as wide or more

Cinara Curtis, 1835 E8

Genus Cinara Curtis, 1835

Subgenus Cinara Curtis, 1835

E8 (E9) E8 (E9) ............. Segment 1 of hind tarsus short; its dorsal side about half the length of the ventral

Cinara (Cinara) Curtis, 1835

Cinara (Cinara) pini (Linnaeus, 1758)

Figs $88-89$

\section{Diagnosis}

Apterae 2-3.5 mm, greyish brown, brown or olive brown, with a bronze sheen and thin but distinct wax markings along mid-line and at segment borders; ventrally more or less completely wax-dusted. Body dorsoventrally flattened, widest anterior to the siphunculi, evenly tapering towards head. Dorsal hairs on abdomen sparse and very short, mostly $0.01-0.04 \mathrm{~mm}$, usually less than 10 hairs between the siphuncular sclerites. See also C. acutirostris (on Pinus nigra and P. pinea) and C. cembrae (on P. cembra). Ovipara (if correctly interpreted) with a large dull black transverse sclerite across segments 5 and 6, encompassing the siphunculi, but not extending forwards as in C. nigritergi. Holocyclic, monoecious on Pinus. Open forests, meadow margins, sea shores etc. Forming small colonies on young shoots, twigs and branches, viviparous colonies almost invariably attended by ants.

\section{Recorded hosts}

Pinaceae: Pinus mugo*, sylvestris*. Records from Pinus banksiana, cembra, contorta, nigra, pinaster, pinea, sibirica, strobus etc. are to be confirmed. According to Eastop (1972) and Blackman \& Eastop (2015), records from hosts other than P. sylvestris are likely to be misidentifications or misapplications of the name pini.

\section{Recorded attendant ants}

Formicinae: Camponotus herculeanus*; Formica aquilonia*, cinerea*, fusca*, lemani*, polyctena*, pratensis*, rufa*, sanguinea*, truncorum*; Lasius niger*

\section{Distribution}

D F N S.

Cinara (Cinara) acutirostris Hille Ris Lambers, 1956

Fig. 90

\section{Diagnosis}

Aptera 2.5-3.5 mm, similar to C. pini (on Pinus sylvestris and P. mugo), but hairs on abdomen longer, there are several rather long hairs up to $0.1 \mathrm{~mm}$ long between the siphuncular cones. Further, the length of RIV+V is 1.2-1.5 times the length of the second tarsal segment (in C. pini less than 1.2). The diagnosis is based on Dransfield \& Brightwell (2015-2016). Holocyclic, monoecious on Pinus.

\section{Recorded hosts}

Pinaceae: Pinus nigra, pinea. 


\section{Recorded attendant ants}

Formicinae: Formica rufa group.

\section{Distribution}

Western, central and southern Europe. Not yet recorded from the Nordic countries.

Cinara (Cinara) cembrae (Seitner, 1936)

Fig. 91

\section{Diagnosis}

Apterae 3.3-4.8 mm, shiny brown, resembling C. pini. Hairs on abdominal tergite 5 short (maximally 0.004-0.04 mm). RIV 0.21-0.29 mm, longer than $2 \times \mathrm{RV}$ (in C. pini $0.14-0.22 \mathrm{~mm}$, about $2 \times \mathrm{RV}$ ). Hairs on basal part of antennal segment VI maximally about $2 \times$ basal diameter of the segment. The diagnosis is based on Pintera (1966) and Blackman \& Eastop (1994). Holocyclic and monoecious on Pinus cembra, forming colonies on bark of two-year-old or older branches. Attended by ants. Records from Pinus peuce, pumila and sibirica refer to other species (Szelegiewicz 1976).

\section{Recorded hosts}

Pinaceae: Pinus cembra.

\section{Recorded attendant ants}

Formicinae: Formica rufa (group?).

\section{Distribution}

Central and eastern Europe, including St. Petersburg area in Russia.

Cinara (Cinara) nigritergi Mamontova-Solukha, 2002

Figs 92-93

\section{Diagnosis}

Apterae 2.5-4 mm, dark brown to black with grey wax markings and a large dull or subshining sclerite encompassing the siphunculi, anteriorly extending to a variable extent, often reaching the thoracal sclerites. Hairs short and sparse, as in C. pini (see above), and in contrast to C. pinihabitans. Open forests on sand or rock, meadow margins, pine plantations, sea shores etc. Holocyclic (ovipara described by Stekolshchikov 2011), monoecious on Pinus, forming colonies on young shoots and 1-2 years old twigs on trees of all ages; usually attended by ants. Ovipara with a preanal wax ring.

\section{Recorded hosts}

Pinaceae: Pinus sylvestris*.

\section{Recorded attendant ants}

Formicinae: Lasius fuliginosus*, niger*.

\section{Distribution}

F S [SWE: Hall., Varberg, Träslövsläge, N57 02.8 E12 17.3, Sea shore dune. Pinus sylvestris. Dense colony on 1-2 year-old branch, attended by Lasius fuliginosus. A06-1162. A. Albrecht]. 
Cinara (Cinara) pinihabitans (Mordvilko, 1895)

Figs 94-95

\section{Diagnosis}

Apterae $3-4.5 \mathrm{~mm}$, fairly similar to $C$. pini but much denser and longer pubescent. Hairs on abdomen $0.1-0.2 \mathrm{~mm}, 40-50$ present between the siphuncular sclerites. A transverse dull brown to black sclerite between the siphuncular cones and extending forwards along the spinal and pleural areas present, at least (always?) in the ovipara. Compare the short-haired C. nigritergi below. Holocyclic, monoecious on Pinus, forming colonies on old branches. Probably attended by ants.

\section{Recorded hosts}

Pinaceae: Pionus mugo, sylvestris*.

\section{Distribution}

F S.

Cinara (Cinara) nuda Mordvilko, 1895

Figs 96-97

\section{Diagnosis}

Apterae 3-4 mm, shiny brown with a bronze sheen and two longitudinal patches or streaks, hardly wax-dusted at all. Dorsal hairs short, strong, erect. Found at forest margins, sea shores, sand dunes, pine plantations etc. Holocyclic, monoecious on Pinus sylvestris, forming large colonies on 2-8 year-old parts of trunks and basal branches, usually on young trees. Almost always attended by ants.

\section{Recorded hosts}

Pinaceae: Pinus mugo, nigra, sylvestris*.

\section{Recorded attendant ants}

Formicinae: Formica aquilonia*, cinerea*, fusca*, polyctena*, truncorum *; Lasius niger*, platythorax*.

\section{Distribution}

F S.

E9 (E8) Hind tarsus 1 long; its dorsal side $2 / 3$ the length of the ventral or more

Cinara (Cinara) Curtis, 1835

\section{Cinara (Cinara) brauni Börner, 1940}

Fig. 98

\section{Diagnosis}

Apterae 2.5-3.8 mm, golden brown covered with a dense, greyish wax powder, except for posterior part of dorsum which is shiny black. Holocyclic, monoecious on Pinus, sometimes anholocyclic (Binazzi \& Roversi 1987). On 0-1 year-old twigs, usually attended by ants. Diagnosis based on Heie (1995), Blackman \& Eastop (1994) and Dransfield \& Brightwell (2015).

\section{Recorded hosts}

Pinaceae: Pinus nigra, rarely sibirica, sylvestris.

\section{Recorded attendant ants}

Formicinae: Formica rufa group. 


\section{Distribution}

S.

\section{Cinara (Cinara) pilosa (Zetterstedt, 1840)}

Figs 99-100

\section{Diagnosis}

Apterae 3-5 mm, yellowish to reddish brown, sometimes with two longitudinal olive-green streaks dorsally. Wax pattern fairly weak but conspicuous. Hairs very long, on dorsum mostly standing in roundish sclerites. Hairs on femora and tibiae originating from small dark spots. Holocyclic, monoecious on Pinus. Found in open forests on sand or rock, forest margins etc., clearly preferring old pine trees. Usually singly or adults surrounded by a few young on twigs, buds, flower and cone axils etc. Sometimes attended by ants.

\section{Recorded hosts}

Pinaceae: Pinus mugo*, nigra, sylvestris*.

\section{Recorded attendant ants}

Formicinae: Lasius niger*; Myrmicinae: Myrmica rubra*.

\section{Distribution}

F N S [NOR: Fn, Alta, Hjemmeluft, 6955' N 23ํ' $6^{\prime}$ E, Aug. 3. 1992, open pine forest, Pinus sylvestris, 1 al. viv. on needle, A. Albrecht leg. (A92-835)]. (See also Stekolshchikov \& Kozlov 2012).

\section{Cinara (Cinara) pinea (Mordvilko, 1895)}

Figs 101-103, 87B

\section{Diagnosis}

Apterae 3-5 mm, orange-brown, red-brown, dark brown to almost black with conspicuous wax pattern and numerous brown or blackish, roundish, hair-bearing sclerites. Legs with at least knees and hind tarsi fuscous. Hairs long and thin, also on abdominal dorsum, those on legs more or less upright, on tibiae as long as or longer than width of tibia. Holocyclic, monoecious on Pinus.

A very variable species, and probably there are more than one species involved (as also suggested by Blackman \& Eastop (2015)). Mostly in open habitats: forest margins, clearings, dunes, rocks, dry meadows etc. On young shoots, terminal twigs, cone and flower bases. In spring often in dense colonies, later on usually in small groups. Almost invariably attended by ants.

\section{Recorded hosts}

Pinaceae: Pinus banksiana*, mugo*, nigra, pinea, ponderosa, sibirica, sylvestris*, spp.

\section{Recorded attendant ants}

Formicinae: Camponotus herculeanus*, ligniperda*; Formica aquilonia*, cinerea*, fusca*, lemani*, pressilabris*, polyctena*, rufa*, truncorum*; Lasius niger*, platythorax*. Myrmicinae: Myrmica rubra*, ruginodis*.

\section{Distribution}

D F N S. 
Cinara (Cinara) piniphila (Ratzeburg, 1844)

Figs 104-105

\section{Diagnosis}

Aptera 2.5-4 mm. Similar to $C$. pinea but hairs much shorter, particularly on abdominal dorsum (not longer than $0.1 \mathrm{~mm}$ ), those on femora and tibiae oblique, about half as long as width of tibia. Dorsal hair-bearing sclerites fewer than in C. pinea. Holocyclic, monoecious on Pinus. In Finland found on young pines in costal dunes, attended by Formica cinerea. Feeding among needles on young shoots and $1-2$ year-old twigs.

\section{Recorded hosts}

Pinaceae: Pinus sylvestris*.

\section{Recorded attendant ants}

Formicinae: Formica cinerea*.

\section{Distribution}

F.

Cinara (Cinara) schimitscheki Börner, 1940

Fig. 106

\section{Diagnosis}

Apterae 4-4.7 mm, broadly oval and flattened, dark brown, covered with wax powder. Dorsal sclerites rounded, their diameter up to $30 \mu \mathrm{m}$. Dorsal hairs blunt, up to $85 \mu \mathrm{m}$. Antennal hairs fairly short, on segment 3 mostly shorter than the diameter of the segment. Holocyclic, monoecious on Pinus, usually P. nigra. Diagnosis based on Pintera (1966) and Scheurer (1976). In spring at twig apices among previous year's needles, later on underside of older branches under bark flakes.

\section{Recorded hosts}

Pinaceae: Pinus mugo, nigra (usually), pinea.

\section{Recorded attendant ants}

Formicinae: Formica sanguinea; Lasius niger, alienus (Scheurer 1976).

\section{Distribution}

Throughout Europe except in the north. Asia.

\section{Cinara (Cinara) hyperophila (Koch, 1855)}

Figs 107-109

\section{Diagnosis}

Apterae 2.5-3.5 mm, shiny dark brown to black with a metallic lustre, which especially in juveniles can be very conspicuous. Head, thorax and venter with a fairly thick grey wax dusting. A spinal wax streak, spreading along the segment borders is usually present, and adult apterae often with rounded presiphuncular wax patches. Legs and antennae predominantly dark brown to black. Abdominal hairs long, erect, most of them not originating from sclerites. Hairs on femora rather short, erect or semierect, those on tibiae short, oblique. Holocyclic, monoecious on Pinus. Found in forest margins, pine plantations, sea shore dunes etc. Forming small colonies on current year's shoots, rarely on older. Usually on small pines. Often ant-attended. 


\section{Recorded hosts}

Pinaceae: Pinus mugo, pinaster, sylvestris*.

\section{Recorded attendant ants}

Formicinae: Formica cinerea*, fusca*, polyctena*, rufa*, sanguinea*, truncorum*; Lasius niger*, platythorax*.

\section{Distribution}

D F S.

E10 (E4) Siphunculi elongate, tubular

Subfamily Aphidinae Latreille, 1802

Tribe Macrosiphini Wilson, 1910

Genus Elatobium Mordvilko, 1914

Elatobium abietinum (Walker, 1849)

\section{Diagnosis}

Aptera 1-2 mm, pale green to yellowish. See under Picea (entry A12, p. 12).

Tribe Aphidini Latreille, 1802

Genus Aphis Linnaeus, 1758

Subgenus Aphis Linnaeus, 1758

Aphis (Aphis) fabae Scopoli, 1763

\section{Diagnosis}

Aptera 1.5-3 mm, dull black. See under Picea (entry A12, p. 12).

E11 (E1) Aphids on roots of Pinus E12

E12 (E13) Apterae 2-6 mm, with brown, grey or blackish colours; siphunculi present as pores elevated on cones. Usually on thick exposed roots

Cinara Curtis, 1835 go to entry point E7 above (p. 34)

E13 (E12) Apterae 0.5-2 mm, pale pinkish, exuding abundant white wax wool; siphunculi absent. On thin roots

Prociphilus Tullgren, 1925

Subfamily Eriosomatinae Kirkaldy, 1905

Tribe Eriosomatini Kirkaldy, 1905

Genus Prociphilus Tullgren, 1925

Subgenus Stagona Koch, 1857

Prociphilus (Stagona) pini (Burmeister, 1835)

Fig. 110

\section{Diagnosis}

Aptera 1-2 mm, cream or pale pinkish, covered by dense wax wool. Holocyclic, dioecious. Alternating between Crataegus (Rosaceae) and thin roots of Pinus. P. pini prefers habitats on well-drained soil, e.g., pine forests on rock and sandy ground, under moss cover and stones, sometimes in nests of Lasius flavus and attended by the ants. See also Albrecht (2015). 


\section{Recorded secondary hosts}

Pinaceae: Pinus contorta, sylvestris*; Pseudotsuga; Tsuga canadensis.

\section{Recorded attendant ants}

Formicinae: Lasius flavus*.

\section{Distribution}

D F N S.

\section{Key F. Aphids on Cupressaceae}

Synopsis

F1 (F7) On aerial parts of the plant

F2 (F5, F6) Siphunculi as pores elevated on low cones (wider than high) ........ Cinara Curtis, 1835 F3

F3 (F4) Hind tibiae uniformly dark, at most slightly lighter in the basal half Cinara (Cupressobium) Börner, 1940 p.40

F4 (F3) Hind tibiae pale or with a pale zone between the dark knee and apex

Cinara (Cupressobium) Börner, 1940 p. 42

F5 (F2 F6) Siphunculi conical, 1.5-2 times as long as wide

Brachycaudus van der Goot, 1913 p. 43

F6 (F2 F5) Siphunculi parallel-sided, more than 4 times as long as wide

F7 (F1) On thin roots, embedded in wax wool Gootiella Tullgren, 1925 p. 44

F1 (F7) On aerial parts of the plant F2

F2 (F5 F6) Siphunculi as pores elevated on low cones (wider than high) Cinara Curtis, 1835 F3

Superfamily Aphidoidea Latreille, 1802

Family Aphididae Latreille, 1802

Subfamily Lachninae Herrich-Schaeffer, 1854

F3 (F4) Hind tibiae uniformly dark, at most slightly lighter in the basal half

Cinara (Cupressobium) Börner, 1940 p.40

Tribe Eulachnini Baker, 1920

Genus Cinara Curtis, 1835

Subgenus Cupressobium Börner, 1940

Cinara (Cupressobium) smolandiae Danielsson \& Carter, 1993

Figs 111-112

\section{Diagnosis}

Apterae 3-4 mm, olive with a weak bronzy sheen and an evenly distributed thin grey wax dusting. Hairs on tibiae fairly short, on hind tibia not longer than $1.5 \times$ width of tibia at middle. Hind tibia longer than $2.2 \mathrm{~mm}$. Juveniles pinkish or olive brown with thinner wax coating and paler legs. Holocyclic, monoecious on Juniperus communis, forming colonies in bark crevices and old wounds caused by the rust fungus Gymnosporangium. Attended by ants.

\section{Recorded hosts}

Cupressaceae: Juniperus communis*. 


\title{
Recorded attendant ants
}

Formicinae: Camponotus sp.; Formica polyctena*, sp.

\section{Distribution}

F S.

\author{
Cinara (Cupressobium) juniperi (De Geer, 1773)
}

Figs 113-115

\section{Diagnosis}

Apterae 2-2.5 mm, rather light reddish brown, rarely olive, with a wax coating of variable thickness and extent, typically leaving two longitudinal stripes bare or with thinner wax. Hairs on tibiae longer than in C. smolandiae, on the outer side of hind tibia longer than $1.5 \times$ width of tibia. Spinopleural sclerites present on meso- and metanotum and abdominal tergites 1-2 (-5). Juveniles with paler legs and usually thinner wax coating. Holocyclic, monoecious on Juniperus and Thuja. Found in open woodland, forest margins and dry meadows, sea shores, rocky habitats; in small colonies on thin twigs, only rarely on thicker branches. Usually ant-attended.

\section{Recorded hosts}

Cupressaceae: Juniperus chinensis, communis*, sabina, squamata, virginiana; Thuja occidentalis.

\section{Recorded attendant ants}

Formicinae: Formica cinerea*, clara*, fusca*, polyctena*, rufa*; Lasius fuliginosus*, niger*. Myrmicinae: Myrmica rubra*.

\section{Distribution}

D F G N S.

Cinara (Cupressobium) mordvilkoi (Pasek, 1954)

Fig. 116

\section{Diagnosis}

Similar to C. juniperi and sometimes difficult to distinguish. Aptera 2.7-3.2 mm. C. mordvilkoi is slightly larger and its wax dusting is thinner and more evenly distributed, posterior part of abdomen often more or less shiny. Colour in both adults and juveniles - at least in Finnish material - greyish olive without reddish tinge. Spinopleural sclerites present on thorax but absent or strongly reduced on abdomen.

Durak (2011) made a DNA study combined with morphological analyses of the two species, confirmed their distinctness, and found some statistically significant distinguishing characters (all apparently related to size): see Table 1.

Holocyclic, monoecious on Juniperus communis. In spring on thin twigs, in summer moving to roots and stems at ground level. Recorded hosts

\section{Recorded hosts}

Cupressaceae: Juniperus communis*.

\section{Distribution}

\section{F.}


Table 1. Morphological features of the apterous viviparous females C. juniperi $(\mathrm{n}=30)$ and C. mordvilkoi $(\mathrm{n}=30)$. Minimum-maximum (mean). Statistical significance: $*=\mathrm{P}<0.05 ; * *=\mathrm{P}<0.01$. Length unit $=\mathrm{mm}$. Data from Durak (2011).

\begin{tabular}{lccl}
\hline Characters & C. juniperi & C. mordvilkoi & \\
\hline body length & $2.1-2.45(2.33)$ & $2.75-3.12(2.94)$ & $* *$ \\
tibia length & $0.9-1.12(1.04)$ & $1.45-1.55(1.5)$ & $* *$ \\
antennae segment III & $0.22-0.35(0.28)$ & $0.32-0.4(0.37)$ & $* *$ \\
antennae segment IV & $0.12-0.15(0.128)$ & $0.15-0.17(0.162)$ & $*$ \\
antennae segment V & $0.15-0.21(0.18)$ & $0.2-0.25(0.21)$ & $*$ \\
antennae segment VI & $0.22-0.26(0.23)$ & $0.25-0.27(0.26)$ & $*$ \\
antennae length & $0.82-1.1(0.96)$ & $1.17-1.22(1.19)$ & $* *$ \\
rostrum length & $0.85-1.0(0.9)$ & $1.32-1.72(1.43)$ & $* *$ \\
\hline
\end{tabular}

F4 (F3) Hind tibiae pale or with a pale zone between the dark knee and apex

Cinara (Cupressobium) Börner, 1940

Cinara (Cupressobium) cupressi (Buckton, 1881)

Fig. 117

\section{Diagnosis}

Apterae 1.5-3.5 mm, olive black with a bronzy sheen. Wax cover thin, anteriorly leaving two diverging bands free; area between siphunculi shiny, with more or less well developed transverse wax stipes. Legs dark; tibiae pale at middle. Basal part of antennal segment VI with 4-6 hairs. Juveniles with paler legs. Holocyclic, partially anholocyclic, monoecious on various Cupressaceae. Parks, hedge rows, forest margins. Forming colonies on foliated branches, often causing damage to the plant. Attended by ants.

\section{Recorded hosts}

Cupressaceae: Chamaecyparis lawsoniana; Cupressus spp; Juniperus chinensis, communis, sabina, scopulorum, virginiana, spp.; Thuja occidentalis*, plicata*.

\section{Recorded attendant ants}

Formicinae: Lasius niger*.

\section{Distribution}

D F S.

Cinara (Cupressobium) fresai (Blanchard, 1939)

\section{Diagnosis}

Similar to C. cupressi, best distinguished by having 7-12 hairs on the basal part of antennal segment VI (4-6 in C. cupressi). See also Carter \& Maslen (1982). Found in unheated glasshouses, apparently anholocyclic. Diagnoses based on Blackman \& Eastop 2015 and Carter \& Maslen 1982.

\section{Recorded hosts}

Cryptomeria japonica; Cupressus; Juniperus chinensis, sabina, squamata, virginiana. 


\section{Distribution}

Britain. Not recorded in the Nordic countries.

Cinara (Cupressobium) tujafilina (Del Guercio, 1909)

Fig. 118

\section{Diagnosis}

Apterae 1.5-3.2 mm, reddish-brown with a whitish dorsal wax coating, leaving two longitudinal stripes and parts of the area around siphunculi bare and shining. Mostly anholocyclic (Blackman \& Eastop 1994) on various Cupressaceae. Tibiae dark only at apices. On foliated and bare branches, wounds, roots. Attended by ants.

\section{Recorded hosts}

Cupressaceae: Chamaecyparis lawsoniana; Cupressus sp.; Juniperus, chinensis, communis, procumbens, virginiana, spp.; Thuja occidentalis; Thujopsis dolabrata.

\section{Recorded attendant ants}

Formicinae: Lasius niger.

\section{Distribution}

Almost cosmopolitan. Not yet recorded from North Europe.

F5 (F2 F6) Siphunculi conical, 1.5-2 times as long as wide... Brachycaudus van der Goot, 1913

Subfamily Aphidinae Latreille, 1802

Tribe Macrosiphini Wilson, 1910

Genus Brachycaudus van der Goot, 1913

Subgenus Brachycaudus van der Goot, 1913

Brachycaudus (Brachycaudus) helichrysi (Kaltenbach, 1843)

\section{Diagnosis}

Aptera 1-1.8 mm, pale green, yellow or yellowish white, ovoid, slightly dorsoventrally flattened. Appendages apically darkened. Siphunculi conical, 1-1.5 times as long as wide. Holocyclic, dioecious, alternating between Prunus (Rosaceae) and Asteraceae and Boraginaceae. Cryptomeria is hardly a true host. Often attended by ants, but no record from conifers.

\section{Recorded coniferous hosts}

Cupressaceae: Cryptomeria japonica?

\section{Distribution}

D F I N S. 
F6 (F2 F5) Siphunculi parallel-sided, more than 4 times as long as wide Myzus Passerini, 1860

Genus Myzus Passerini, 1860

Subgenus Nectarosiphon Schouteden, 1901

Myzus (Nectarosiphon) persicae (Sulzer, 1776)

\section{Diagnosis}

Aptera 1.2-2.2 mm, almond-shaped, pale yellow to dirty yellow, olive, green, brownish, purple or reddish. For image, see e.g., Albrecht (2015). Siphunculi twice as long as cauda or more, slightly swollen beyond middle. Dioecious, alternating between peach (Prunus persica) and plants of more than 40 families. Anholocyclic in the north and in the tropics. Usually in urban environments, also a common indoor pest. Thuja is hardly a true host.

\section{Recorded coniferous hosts}

Cupressaceae: Thuja plicata?

\section{Distribution}

D F N S.

F7 (F1) On thin roots, embedded in wax wool Gootiella Tullgren, 1925

Subfamily Eriosomatinae Kirkaldy, 1905

Tribe Pemphigini Herrich-Schaeffer, 1854

Genus Gootiella Tullgren, 1925

Gootiella tremulae Tullgren, 1925

For key and diagnosis, see Albrecht (2015): 8, key A.

\section{Recorded hosts}

Holocyclic, dioecious, alternating between Populus tremula (Salicaceae) and Juniperus (Cupressaceae), where the aphids live in wax nests similar to those of P. lactea, and commonly over winter there (Danielsson 1990).

\section{Recorded secondary hosts}

Cupressaceae: Juniperus communis, sabina.

\section{Distribution}

D F S.

\section{Acknowledgements}

As in the first paper in this series (Albrecht 2015) I wish to express my gratitude to the Ministry of the Environment and the Finnish Museum of Natural History for support and encouragement. My sincere thanks are also due to members of the Finnish expert group on Hemiptera, my friend Jouni Issakainen who continuously challenges me with intricate questions emanating from his keen interest in the interaction between plants and aphids, and for many enjoyable field excursions. And, again, I want to thank my wife Margareta for her everlasting patience. My friend and colleague Lauri Kaila kindly commented on the manuscript and suggested valuable improvements. I also wish to thank two anonymous referees for valuable comments and suggestions. 


\section{References}

Albrecht A. 2010. Atlas of the Aphids of Finland. Available from http://koivu.luomus.fi/elaintiede/ kirvat/atlas.htm [accessed 6 Apr. 2016].

Albrecht A.C. 2015. Identification guide to Nordic aphids associated with mosses, horsetails and ferns (Bryophyta, Equisetophyta, Polypodiophyta) (Insecta, Hemiptera, Aphidoidea). European Journal of Taxonomy 145: 1-55. https://doi.org/ 10.5852/ejt.2015.145

Annand P.N. 1928. A contribution toward a monograph of the Adelginae (Phylloxeridae) of North America. Leland Stanford Junior University Biological Sciences 6: 1-146.

Binazzi A. \& Roversi P.F. 1987. Contributi alla conoscenza degli afidi delle conifere VIII. Modificazioni delle sclerotizzazioni dorsali dell'addome nel corso dell'anno solare in Cinara brauni Börner (Homoptera Aphidoidea Lachnidae). Redia 70: 51-75.

Binazzi A. \& Scheurer S. 2009. Atlas of Honeydew-Producing Conifer Aphids of Europe. Aracne editrice, Rome.

Blackman R.L. \& Eastop V.F. 1994. Aphids on the World's Trees. CAB International, Wallingford.

Blackman R.L. \& Eastop V.F. 2006. Aphids on the World's Herbaceous Plants and Shrubs (2 vols). Wiley, Chichester.

Blackman R.L. \& Eastop V.F. 2015. Aphids on the World's Plants. Available from http://www. aphidsonworldsplants.info [accessed 14 Dec. 2015].

Blackman R.L. \& Eastop V.F. 2016. Aphids on the World's Plants. Available from http://www. aphidsonworldsplants.info [accessed 7 Jan. 2016].

Börner C. 1908. Eine monographische Studie über die Chermiden. Arbeiten aus der Biologischen Bundesanstalt für Land- und Forstwirtschaft 6: 81-320.

Börner C. \& Heinze K. 1957. Aphidina - Aphidoidea. In: Sorauer P. (ed.) Handbuch der Pflanzenkrankheiten 5: 1-402.

Carter C.I. 1971. Conifer woolly aphids (Adelgidae) in Britain. Forestry Commission Bulletin 42: 1-51.

Carter C.I. 1976. A gall forming adelgid (Pineus similis) new to Britain with a key to the adelgid galls on Sitka Spruce. Entomologist's Monthly Magazine 111: 29-32 (1975).

Carter C.I. \& Maslen N.R. 1982. Conifer lachnids. Forestry Commission Bulletin 58: 1-75.

Czechowski W., Radchenko A., Czechowska W. \& Vepsäläinen K. 2012. Fauna Poloniae. Vol. 4 (new series): The Ants of Poland with Reference to the Myrmecofauna of Europe. PAN Warzawska Drukarnia Naukowa, Warsaw.

Danielsson R. 1990. The life cycle of Gootiella tremulae Tullgren, with descriptions of all viviparous morphs and a redescription of Gootiella alba Shaposhnikov. Entomologica Scandinavica 20: 413-428. https://doi.org/10.1163/187631289X00393

Douwes P., Abenius J., Cederberg B. \& Wahlstedt U. 2012. Steklar: Myror-getingar. Hymenoptera: Formicidae-Vespidae. Nationalnyckeln till Sveriges flora och fauna. ArtDatabanken SLU, Uppsala.

Dransfield B. \& Brightwell B. 2015-2016. Aphid genera. Available from http://influentialpoints.com/ Gallery/Aphid_genera.htm [accessed 31 May 2017].

Durak R. 2011. Molecular and morphological identification of Cinara juniperi and Cinara mordvilkoi. Bulletin of Insectology 64 (2): 195-199. 
Eastop V.F. 1972. A taxonomic review of the species of Cinara Curtis occurring in Britain. Bulletin of the British Museum, natural History (Entomology) 27: 104-186.

Eichhorn O. 1956. Eine dritte gefährliche Tannenlaus der Gattung Dreyfusia in Südbaden. Forschungen und Fortschritte 30: 289-293.

Favret C. 2016. Aphid Species File. Available from http://aphid.speciesfile.org/ [accessed 10 Mar. 2016].

Favret C., Havill N.P., Miller G.L., Sano M. \& Victor B. 2015. Catalog of the adelgids of the world (Hemiptera, Adelgidae). ZooKeys 534: 35-54. https://doi.org/10.3897/zookeys.534.6456

Francke-Grosmann H. 1938. Zur Morphologie und Biologie von Pineus sibiricus Chol. ( = cembrae (Chol.) Annand). Tharandt forstliches Jahrbuch 89: 401-424.

Heie O.E. 1980. The Aphidoidea of Fennoscandia and Denmark I. Mindaridae, Hormaphidae, Thelaxidae, Anoecidae and Pemphigidae. Fauna Entomologica Scandinavica 9. Scandinavian Science Press, Klampenborg.

Heie O.E. 1986. Fauna Entomologica Scandinavica. Vol. 17: The Aphidoidea of Fennoscandia and Denmark III. Pterocommatinae and Aphidinae, Aphidini. E.J. Brill/Scandinavian Science Press, LeidenCopenhagen.

Heie O.E. 1992. Fauna Entomologica Scandinavica. Vol. 25: The Aphidoidea of Fennoscandia and Denmark IV. Aphidinae. Part 1 of Tribe Macrosiphini of Subfamily Aphidinae. E.J. Brill, Leiden-New York-Köln.

Heie O.E. 1994. Fauna Entomologica Scandinavica. Vol. 28: The Aphidoidea of Fennoscandia and Denmark V. Aphidinae. Part 2 of Tribe Macrosiphini of Subfamily Aphidinae. E.J. Brill, Leiden-New York-Köln.

Heie O.E. 1995. Fauna Entomologica Scandinavica. Vol. 31: The Aphidoidea of Fennoscandia and Denmark VI. Aphidinae. Part 3 of Macrosiphini and Lachnidae. E.J. Brill, Leiden-New York-Köln.

Heie O.E. 2004. Danmarks Fauna. Vols. 87-88: Bladlus 1-2. Dansk Naturhistorisk Forening, Copenhagen.

Holman J. 2009. Host Plant Catalog of Aphids. Palaearctic Region. Springer Science + Business Media B.V, the Netherlands.

Inouye M. 1953. Monographische Studie über die japanischen Koniferen-Gallenläuse (Adelgidae). Bulletin of the Sapporo Government Forest Experiment Station 15: 1-91.

Karlsson T. \& Agestam M. 2014. Checklista över Nordens kärlväxter. [Checklist of vascular plants of Scandinavia]. Available from http://www.euphrasia.nu/checklista/ [accessed 12 Sep. 2015].

Pashchenko N.F. 1988. Aphidinea. In: Lehr P.A. (ed.) Keys to the Insects of the Far East of the USSR. Vol. 2. Nauka Publishing House, Leningrad. (English translation: 2001, U.S. Department of Agriculture).

Pschorn-Walcher H. \& Zwölfer H. 1958. Preliminary investigations on the Dreyfusia (Adelges) populations, living on the trunk of the Silver Fir. Zeitschrift für angewandte Entomologie 42: 241-277. https://doi.org/10.1111/j.1439-0418.1958.tb00896.x

Pintera A. 1966. Revision of the genus Cinara Curt. (Aphidoidea, Lachnidae) in Middle Europe. Acta Entomologica Bohemoslovaca 63: 281-321.

Shaposhnikov G.K. 1964. Aphidinea - Plant lice. In: Bei-Bienko G.Y. (ed.) Keys to the insects of the European USSR. Vol. 1: Apterygota, Palaeoptera, Hemimetabola. Izdastel'stvo "Nauka", MoscowLeningrad. (English translation: 1967, Israel Program for Scientific Translations, Jerusalem). 
ALBRECHT A.C., Identification guide to Nordic aphids on conifers

Scheurer S. 1976. Cinara schimitscheki Börner (Homoptera, Lachnidae) - Angaben zur Morphologie und Biologie. Annales Zoologici 33: 261-271.

Schneider-Orelli O. 1940. Die weisswollige Fichtenstammlaus Pineus (Cherme) Pineoides Cholod. Schweizerische Zeitschrift für Forstwesen 11: 4-8.

Schneider-Orelli O. 1947. Entomologisches Praktikum. Einführung in die land-und forstwirtschaftliche Insektenkunde. Aarau, Sauerländer.

Stekolshchikov A.V. 2011. Description of oviparous female of Cinara nigritergi Mamontova, 2002 (Hemiptera: Aphididae). Entomologica Fennica 22: 209-212.

Stekolshchikov A.V. \& Kozlov M.V. 2012. Distribution records of aphids (Hemiptera: Phylloxeroidea, Aphidoidea) associated with main forest-forming trees in Northern Europe. Entomologica Fennica 23: 206-214.

Szelegiewicz H. 1976. Aphid species new to the Polish fauna. Annales Zoologici 33: 217-227.

Manuscript received: 20 May 2016

Manuscript accepted: 22 September 2016

Published on: 24 July 2017

Topic editor: Gavin Broad

Desk editor: Chloe Chester

Printed versions of all papers are also deposited in the libraries of the institutes that are members of the EJT consortium: Muséum national d'Histoire naturelle, Paris, France; Botanic Garden Meise, Belgium; Royal Museum for Central Africa, Tervuren, Belgium; Natural History Museum, London, United Kingdom; Royal Belgian Institute of Natural Sciences, Brussels, Belgium; Natural History Museum of Denmark, Copenhagen, Denmark; Naturalis Biodiversity Center, Leiden, the Netherlands; Museo Nacional de Ciencias Naturales-CSIC, Madrid, Spain; Real Jardín Botánico de Madrid CSIC, Spain. 


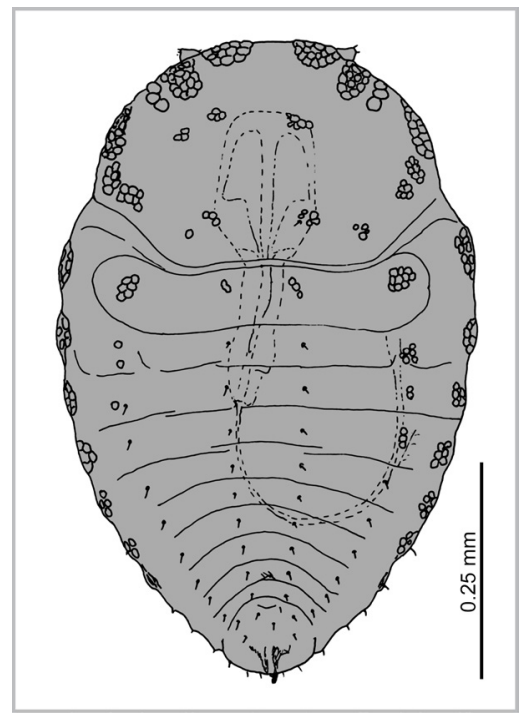

Fig. 1. Pineus (Pineus) pineoides (Cholodkovsky, 1907). Adult aptera (after Schneider-Orelli 1940, modified).

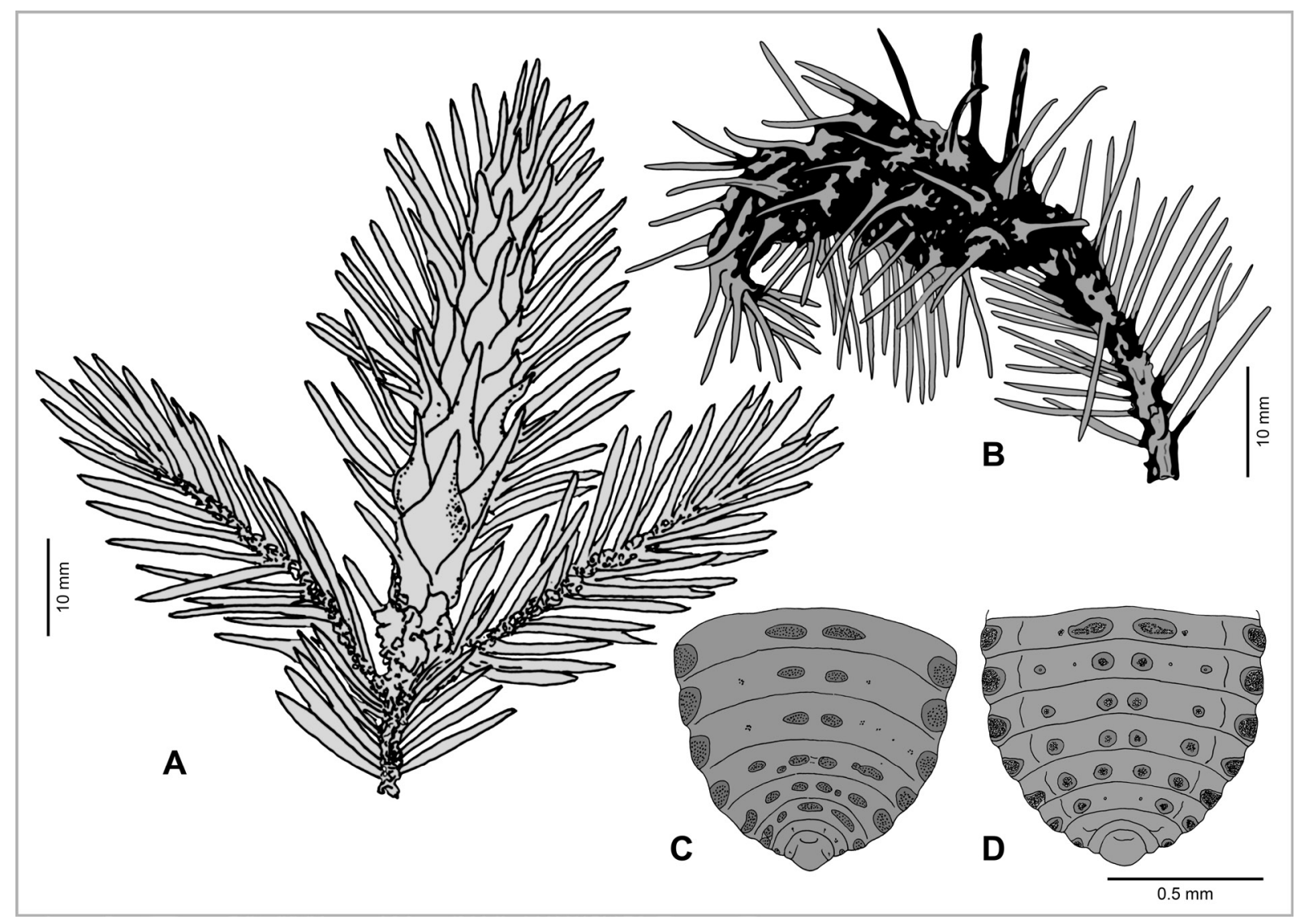

Fig. 2. Pineus (Pineus) cembrae (Cholodkovsky, 1888). A-B. Galls on Picea abies (A after Francke-Grosmann 1938, redrawn; B after Shaposnikov, modified from Heie 2004). C-D. Gallicola (C) and sexupara (D), abdomen showing wax gland plates (after Börner 1908, modified from Heie 2004). 

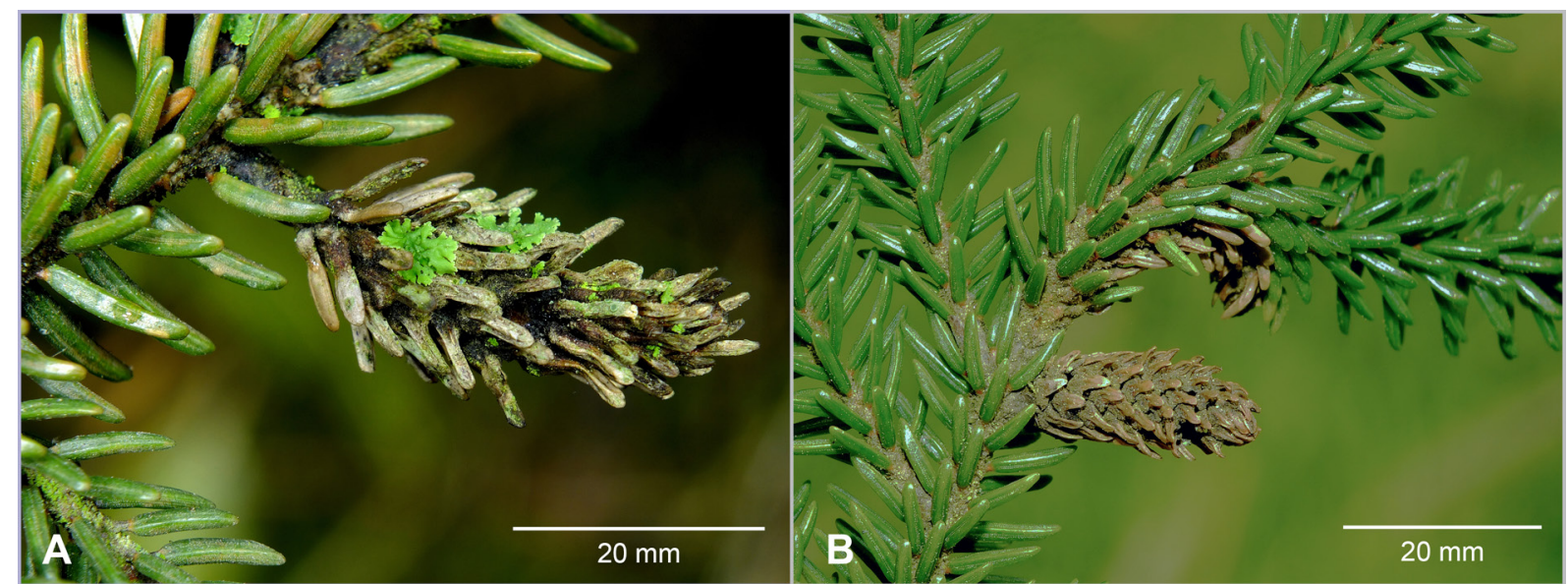

Fig. 3. Pineus (Pineus) orientalis (Dreyfus, 1889), old galls on Picea orientalis.

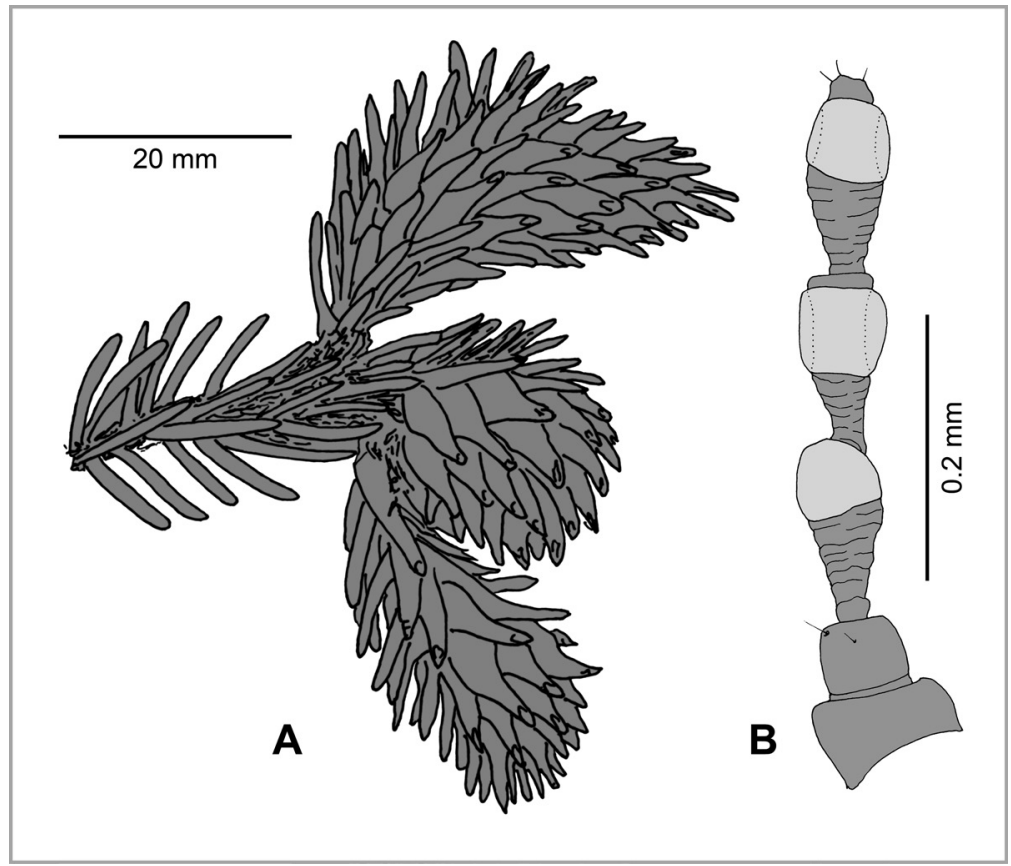

Fig. 4. Pineus (Pineus) orientalis (Dreyfus, 1889). A. Gall on Picea orientalis (after Carter 1976, redrawn). B. Antenna of gallicola (after Annand 1928, modified).

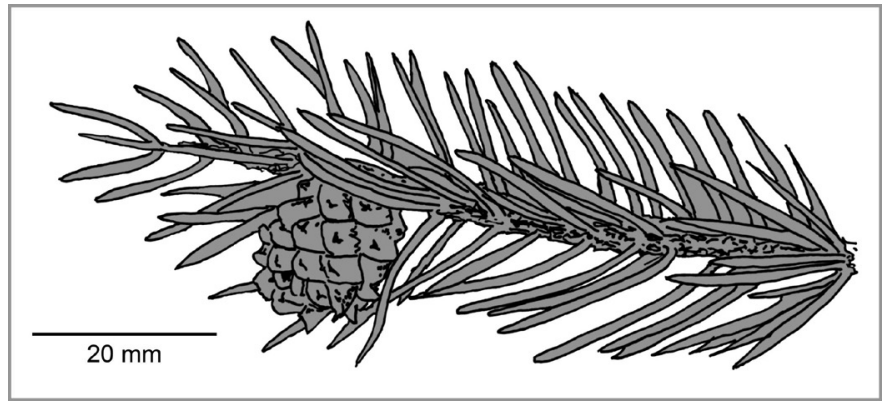

Fig. 5. Adelges (Aphrastasia) pectinatae (Cholodkovsky, 1888). Gall on Picea abies (after Cholodkovsky, redrawn and modified from Shaposhnikov 1964). 


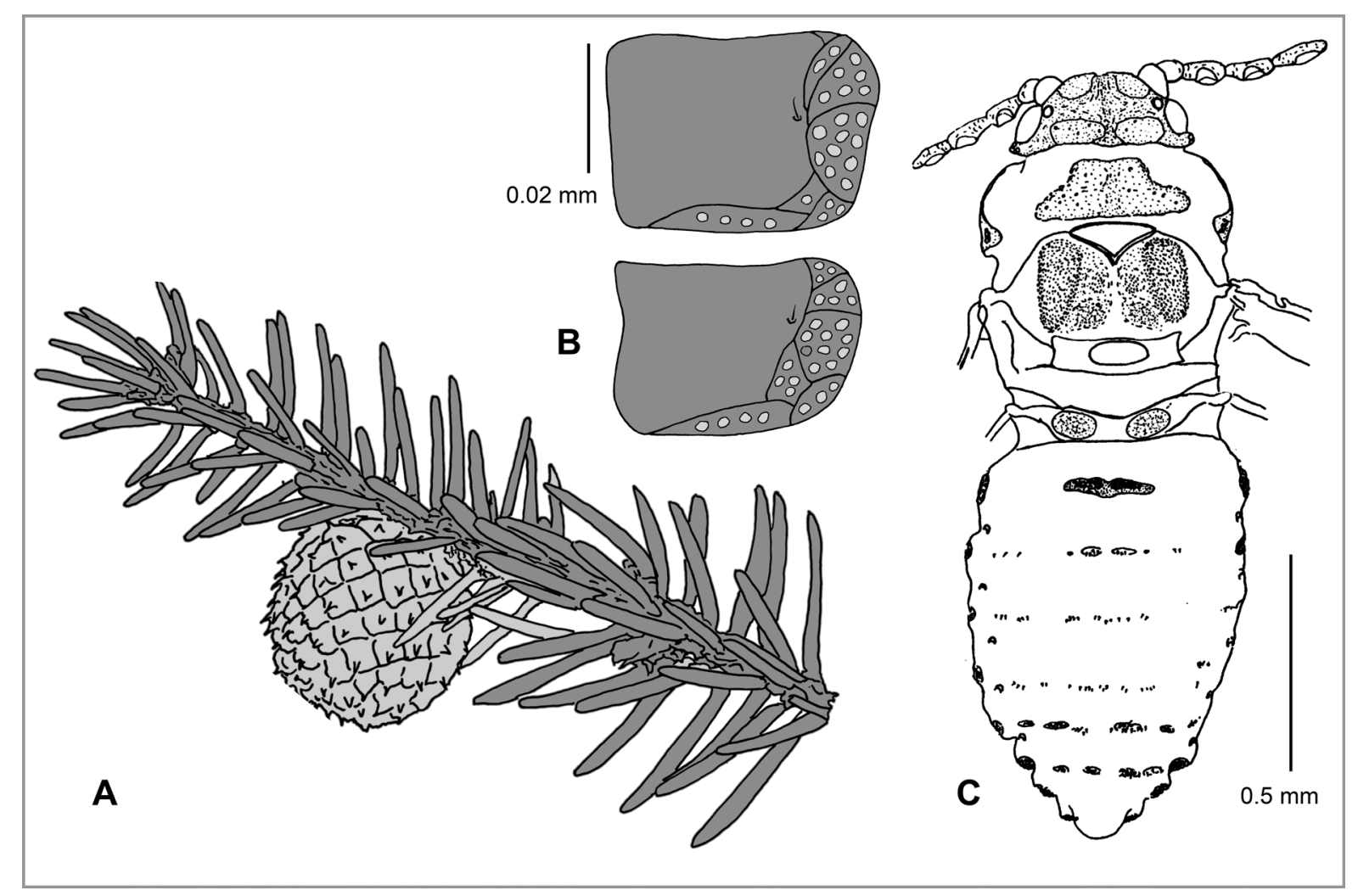

Fig. 6. Adelges (Dreyfusia) nordmannianae (Eckstein, 1890). A. Gall on Picea orientalis (orig.). B. First instar hibernating fundatrix, spinal plates on meso- and metathorax (left side), showing arangement of wax gland pits (after Eichhorn 1956). C. Gallicola (after Carter 1971, redrawn and slightly modified from Heie 2004).

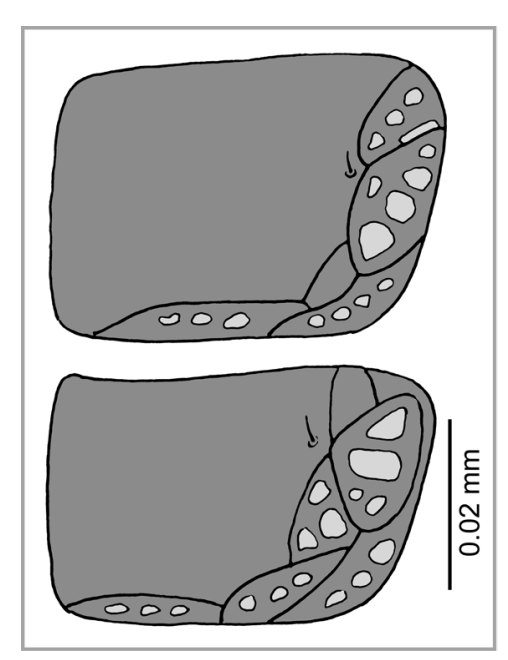

Fig. 7. Adelges (Dreyfusia) merkeri (Eichhorn, 1957). First instar hibernating fundatrix, spinal plates on meso- and metathorax (left side), showing arangement of wax gland pits (after Eichhorn 1956, modified). 


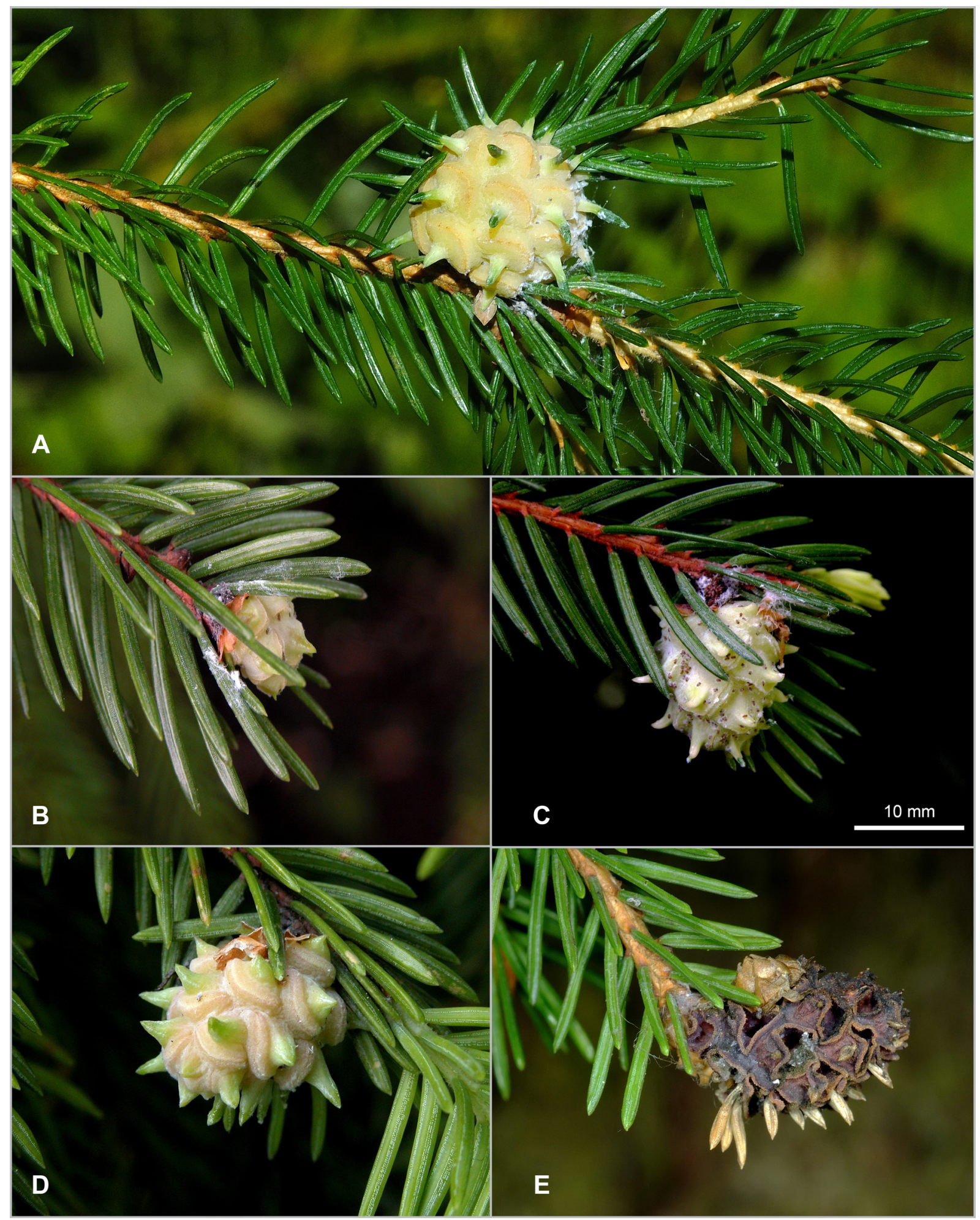

Fig. 8. Adelges (Adelges) laricis Vallot, 1836. Galls on Picea abies. A, D. Almost mature. B. Young. C. Half-grown. E. Abandoned gall (in winter). 


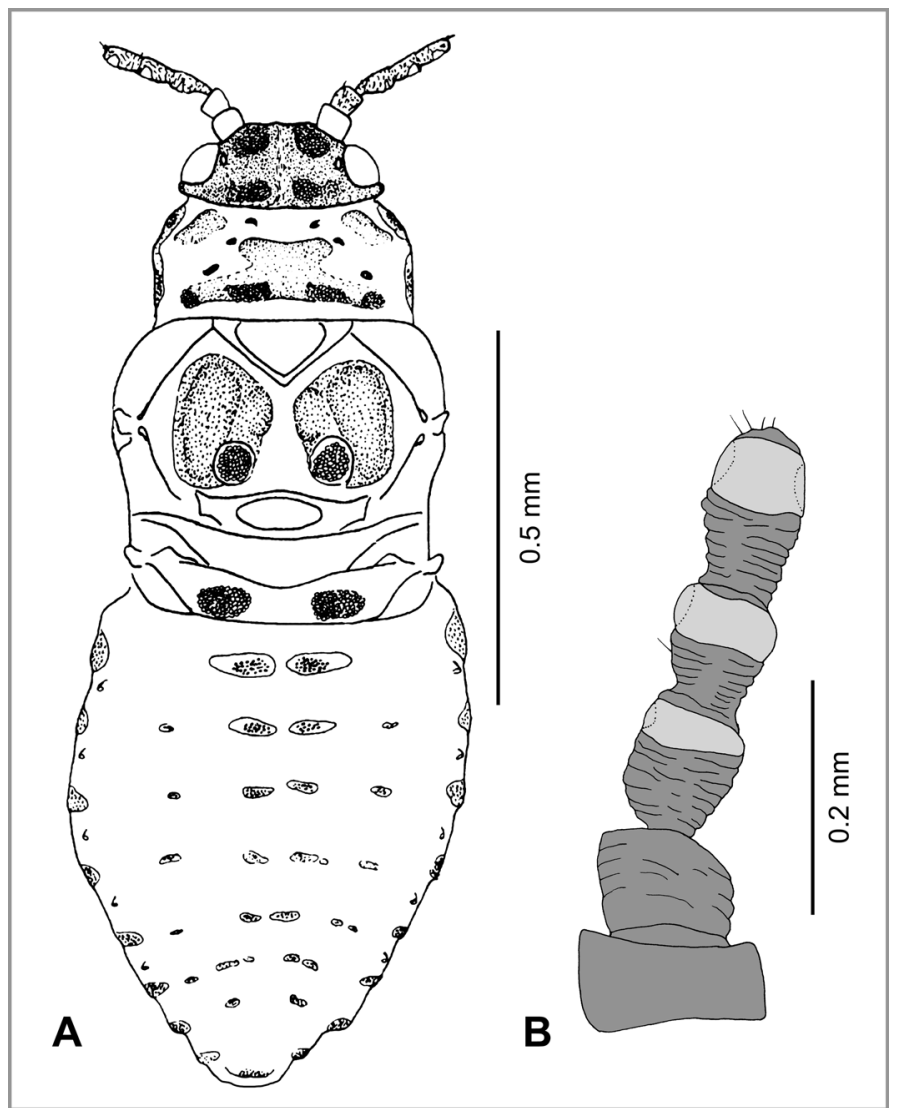

Fig. 9. Adelges (Adelges) laricis Vallot, 1836. A. Sexupara (after Carter 1971, modified and redrawn from Heie 2004). B. Antenna of gallicola (after Annand 1928, modified). 


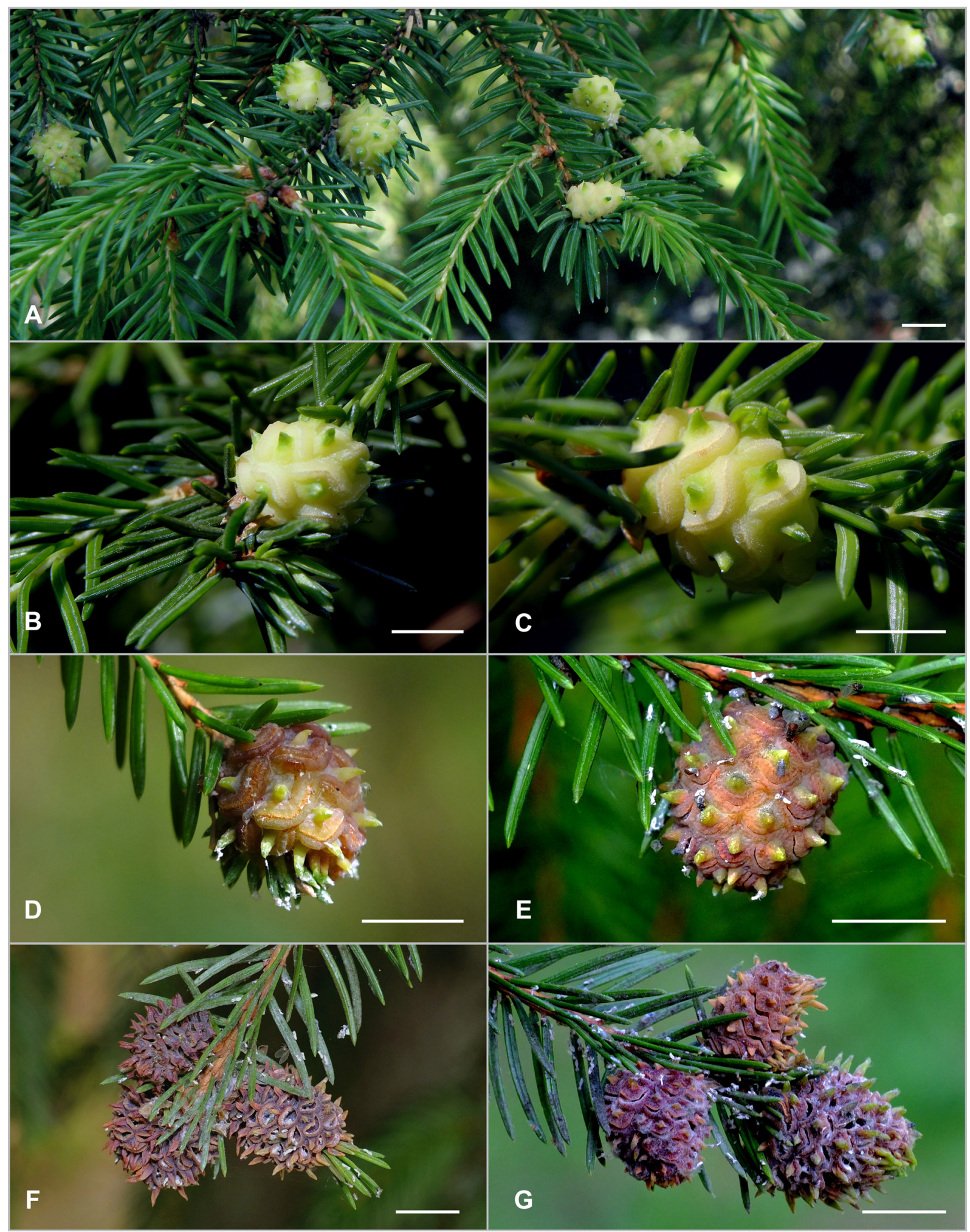

Fig. 10. Adelges (Adelges) tardus (Dreyfus, 1888). Galls on Picea abies. A-B. Almost full-grown. C. Almost mature. D-E. Mature, first gallicolae have emerged. F-G. Fully opened galls. Scale bars: $10 \mathrm{~mm}$. 


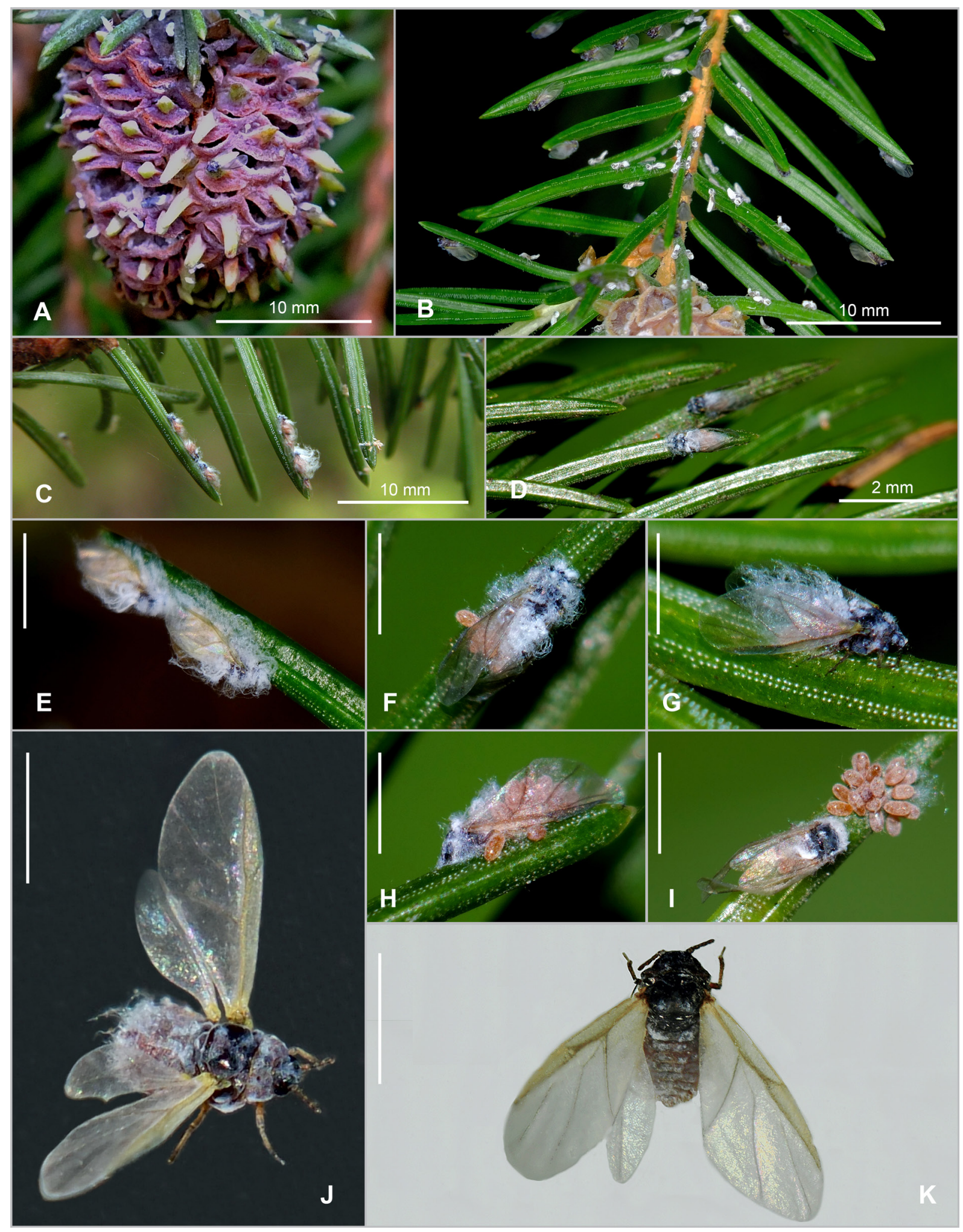

Fig. 11. Adelges (Adelges) tardus (Dreyfus, 1888) on Picea abies. A. Mature, opened gall with alate gallicolae on surface. B-I. Gallicolae laying eggs on needles in vicinity of their mother gall. $\mathbf{J}-\mathbf{K}$. Gallicolae in vitro. Scale bars for E-K: $1 \mathrm{~mm}$. 

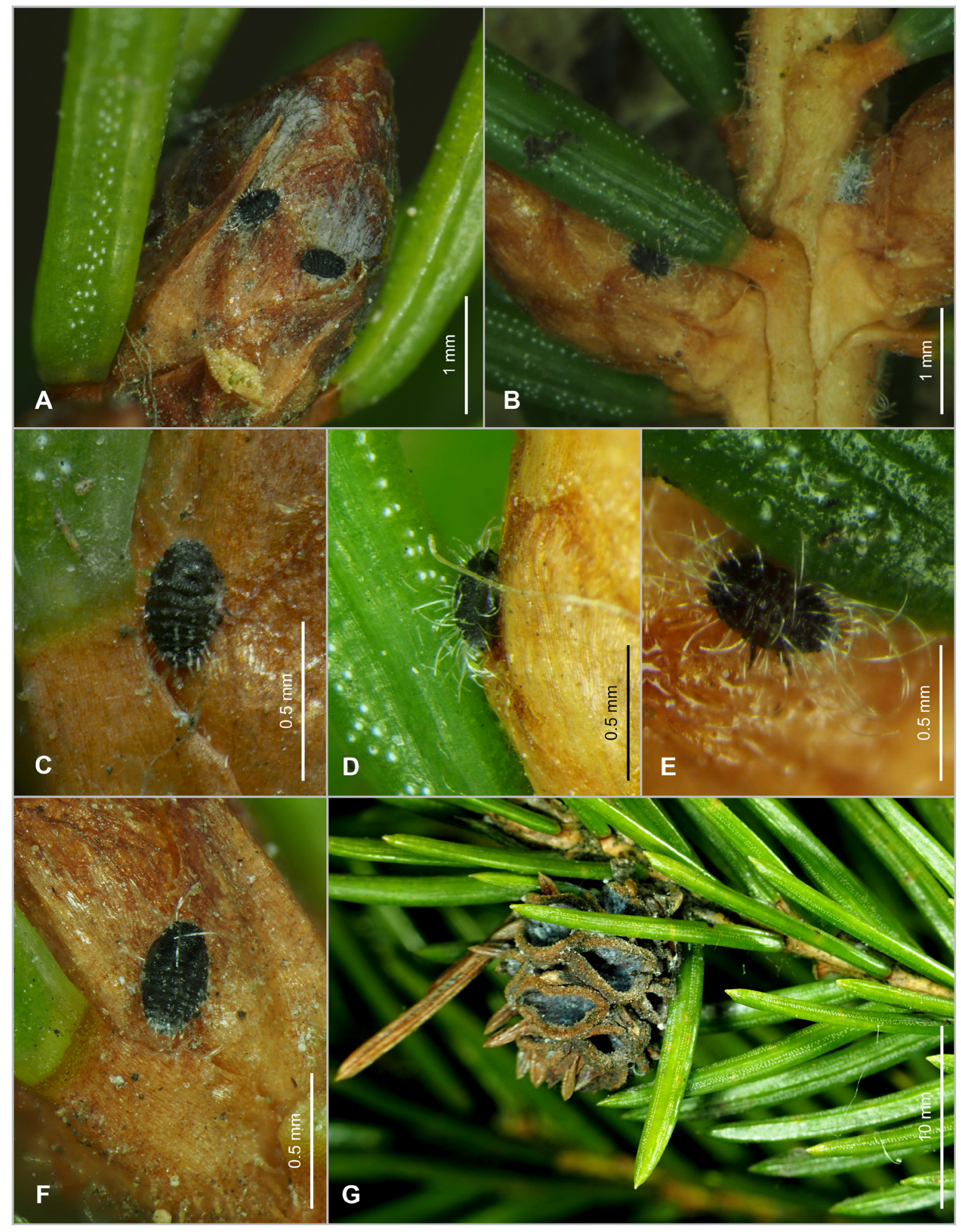

Fig. 12. Adelges (Adelges) tardus (Dreyfus, 1888). A-F. Hibernating pseudofundatrix nymphs on buds of Picea abies. Note the sparse, long, more or less straight wax filaments (most of them worn off, except in D-E). Often together with nymphs of A. (Sacciphantes) abietis (Linnaeus, 1758), whose wax filaments are dense, flattened and strongly curled (the specimen to the right in B). The nymphs usually hibernate on the surface of the bud, whereas those of A. abietis prefer bud bases and axils. G. Hibernating nymphs, which are easiest to find in the vicinity of the previous summer's galls. 

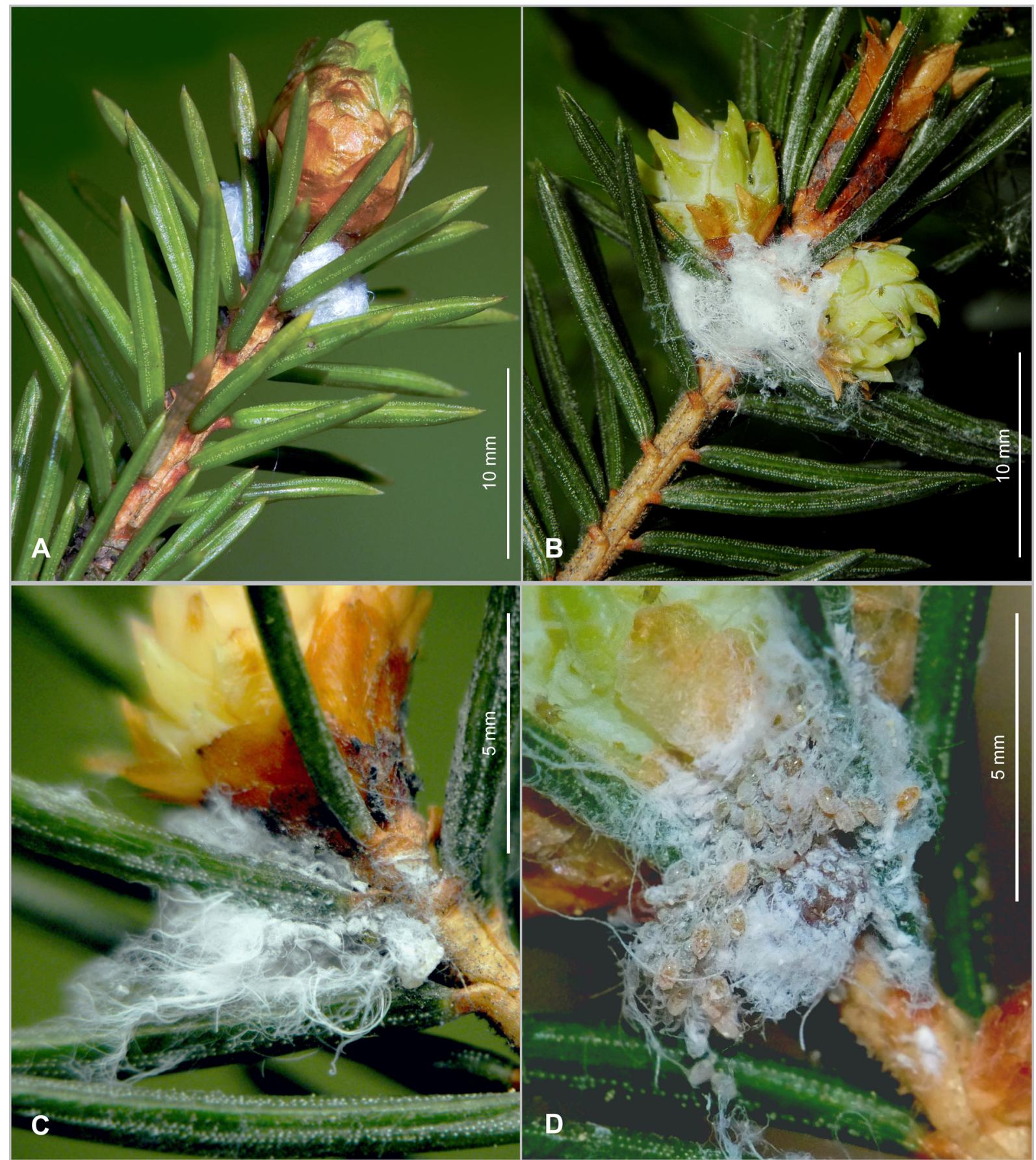

Fig. 13. A-C. Adelges (Adelges) tardus (Dreyfus, 1888). Wax-covered pseudofundatrices at bases of developing galls on Picea abies. D. As A-C, but with the wax wool brushed off to expose the pseudofundatrix and her eggs. 


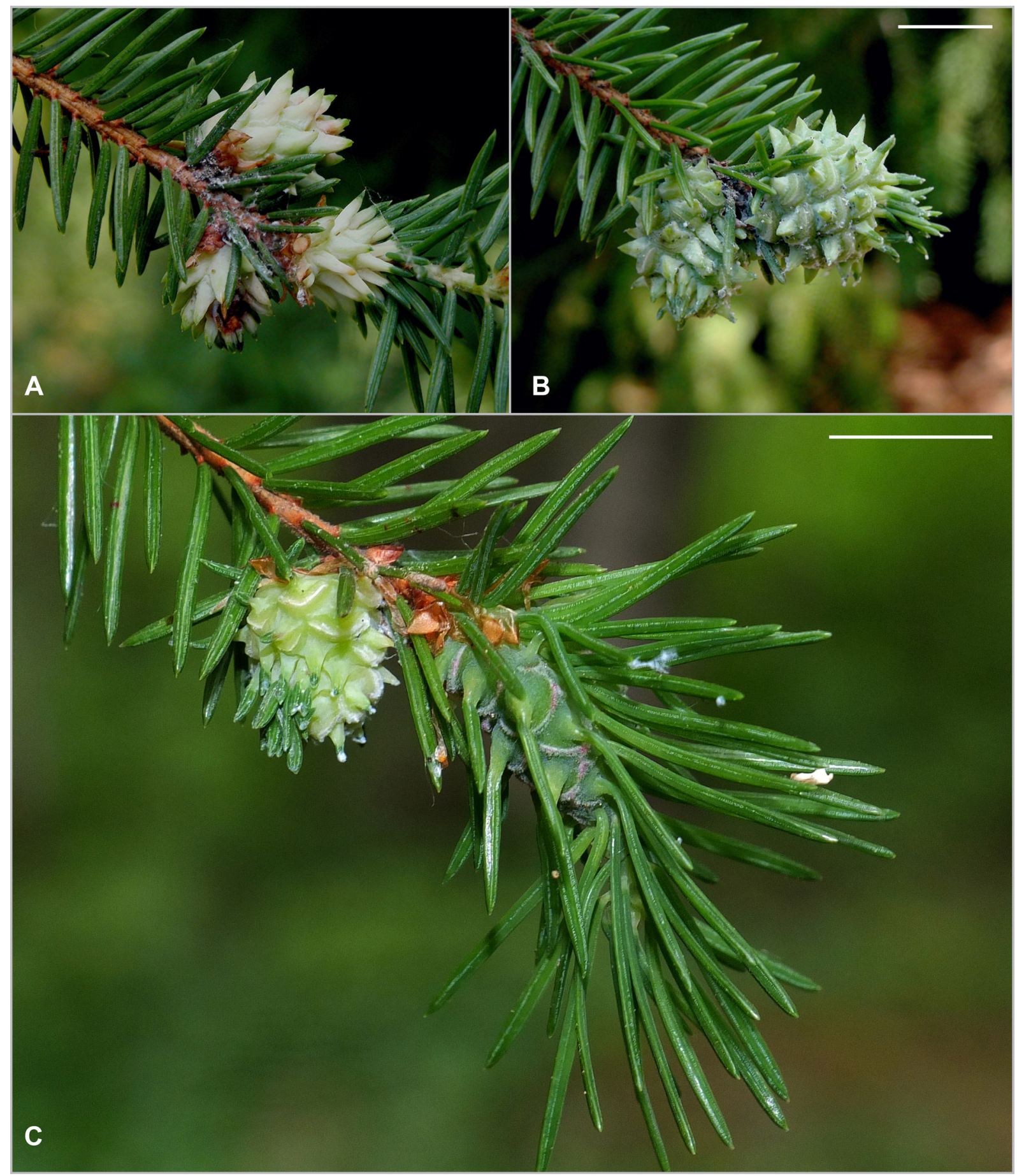

Fig. 14. A-B. Developing galls of Adelges (Adelges) tardus (Dreyfus, 1888) on Picea abies, close to galls of A. (Sacciphantes) abietis (Linnaeus, 1758). C. Half-grown galls of A. tardus and A. abietis on the same twig. Note the tufts of protruding needles on the A. tardus galls, apparently due to interference from $A$. abietis. Scale bars: $10 \mathrm{~mm}$. 


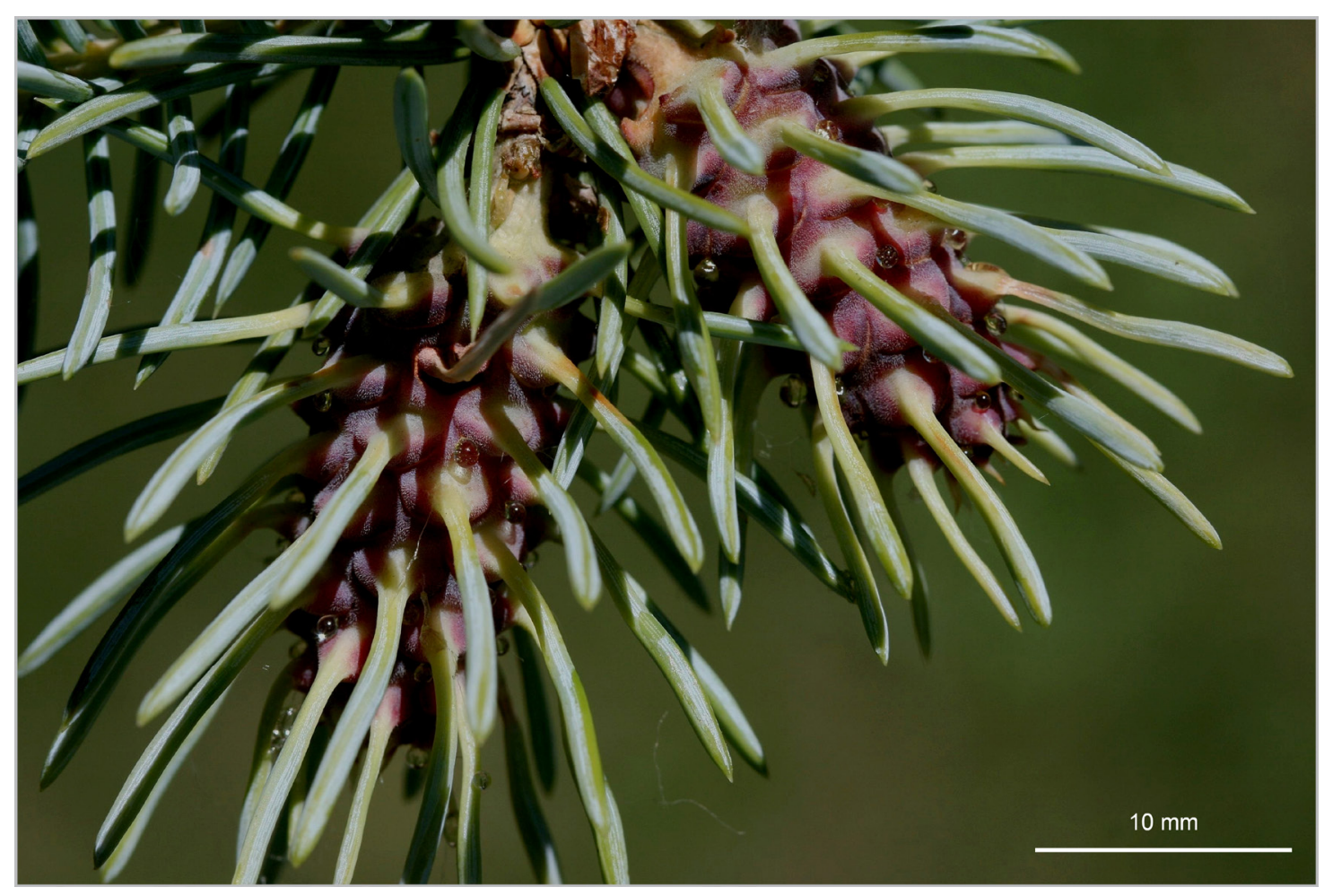

Fig. 15. Adelges (Gilletteella) cooleyi (Gillette, 1907). Galls on Picea pungens. Photo Whitney Cranshaw, Colorado State University, Bugwood.org (CC BY 3.0 US, https://creativecommons.org/licenses/by/3.0/ us/, downloaded via http://www.forestpests.org/vd/images/5422254-SMPT.jpg).
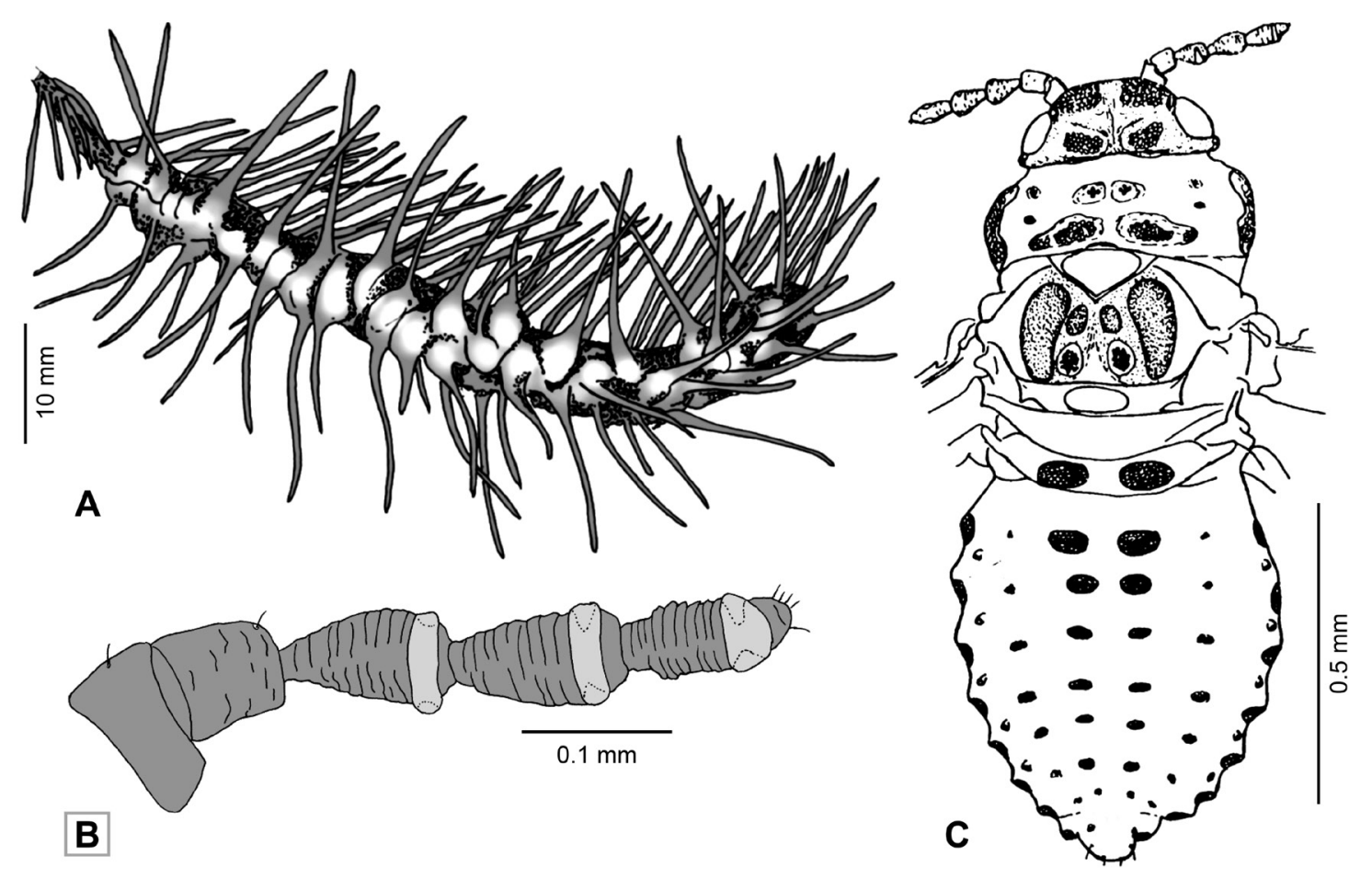

Fig. 16. Adelges (Gilletteella) cooleyi (Gillette, 1907). A. Gall on Picea (after Carter 1976). B. Antenna of gallicola (modified from Annand 1928). C. Sexupara (after Carter 1971, redrawn and modified from Heie 2004). 

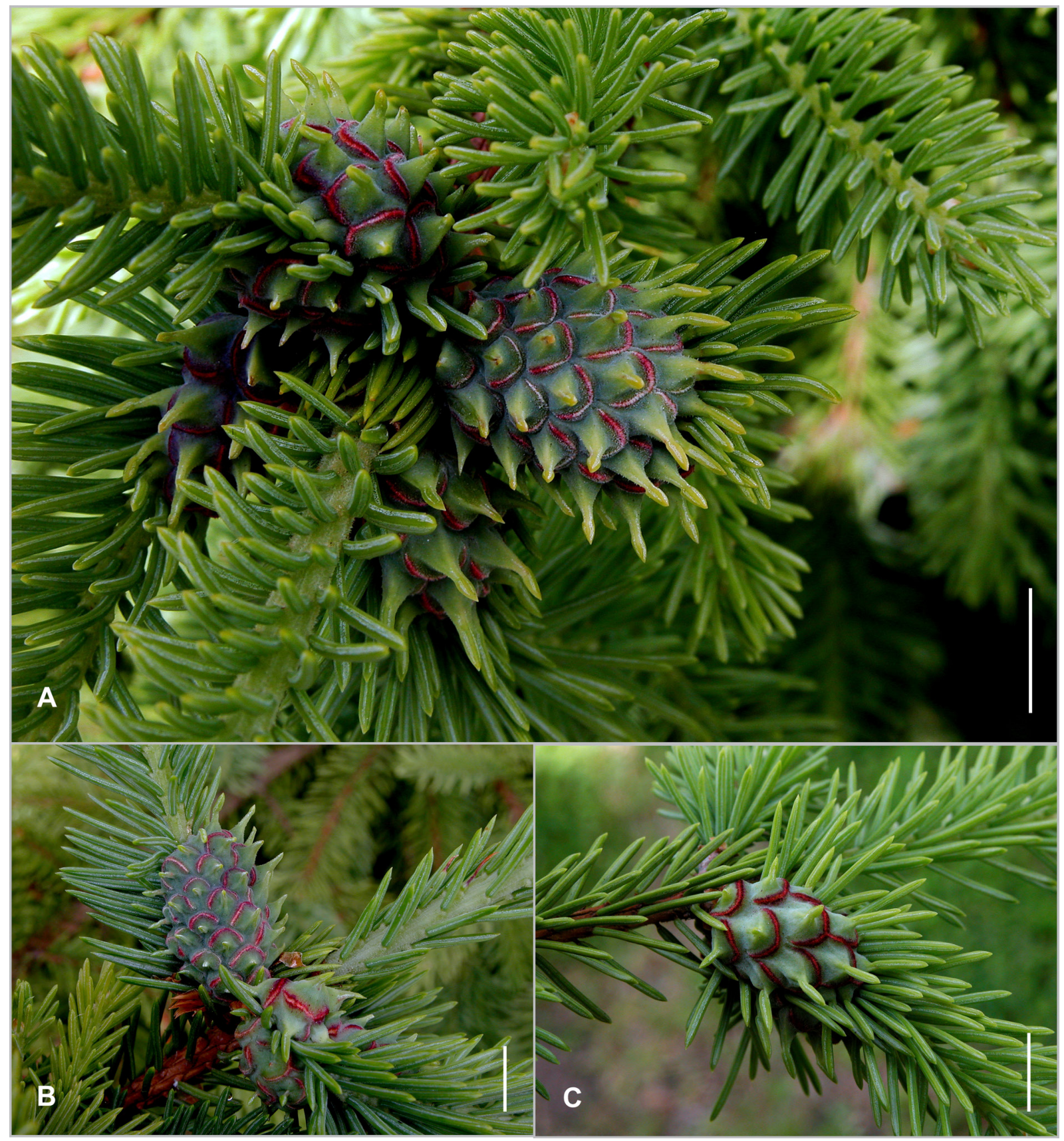

Fig. 17. Adelges (Sacciphantes) abietis (Linnaeus, 1758), galls on Picea abies. A-B. Almost full-grown. C. Half-grown. Colouration typical for galls growing in open sites. Scale bars: $10 \mathrm{~mm}$. 


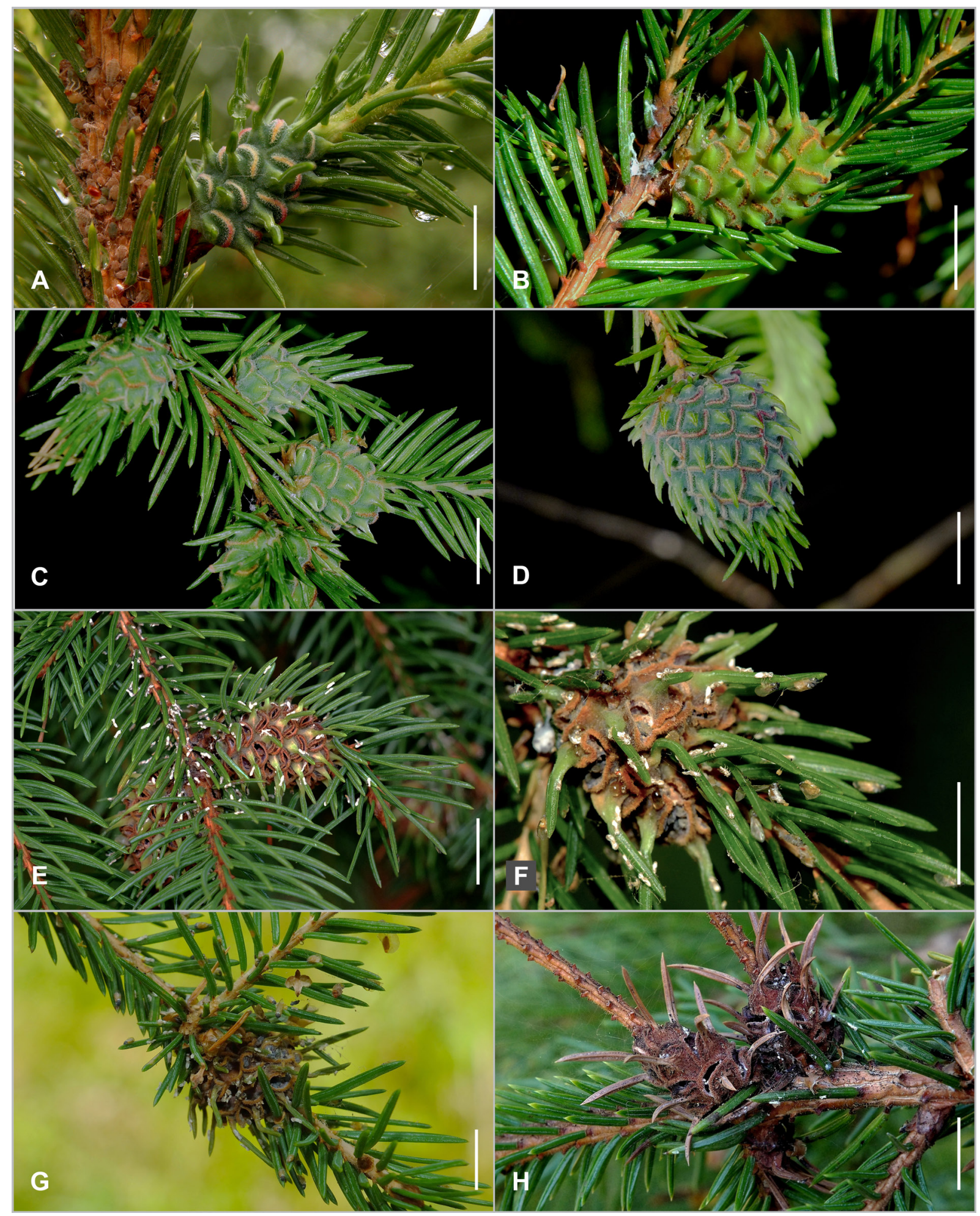

Fig. 18. Adelges (Sacciphantes) abietis (Linnaeus, 1758), galls on Picea abies. A-D. Galls from different sites showing variation in colouration. A. From a semi-open site. B. In semi-shade. C-D. In deep shade. E-F. Newly opened galls with exuviae and alate gallicolae around. G-H. Galls from previous summer. Note the colony of Cinara pilicornis (Hartig, 1841) on the stem in A. Scale bars: $10 \mathrm{~mm}$. 

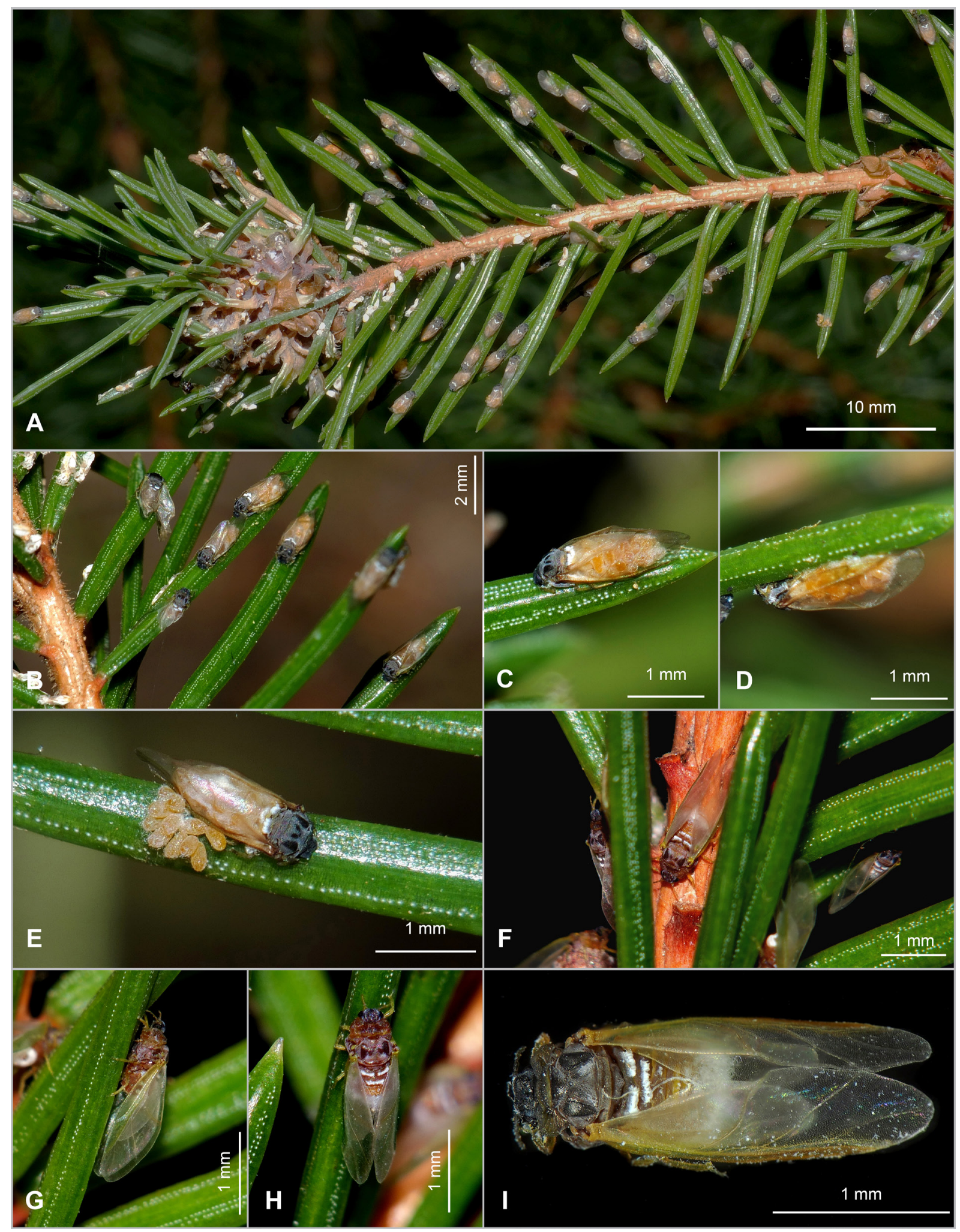

Fig. 19. Adelges (Sacciphantes) abietis (Linnaeus, 1758) on Picea abies. The gallicolae fly only a very short distance from their mother gall (A-B), where they lay their eggs $(\mathbf{C}-\mathbf{E})$. A majority of the eggs remain on the mother's dorsum, sheltered by her wings. Newly hatched alatae are red-brown $(\mathbf{F}-\mathbf{G})$, head and thorax darkening with age (I). 

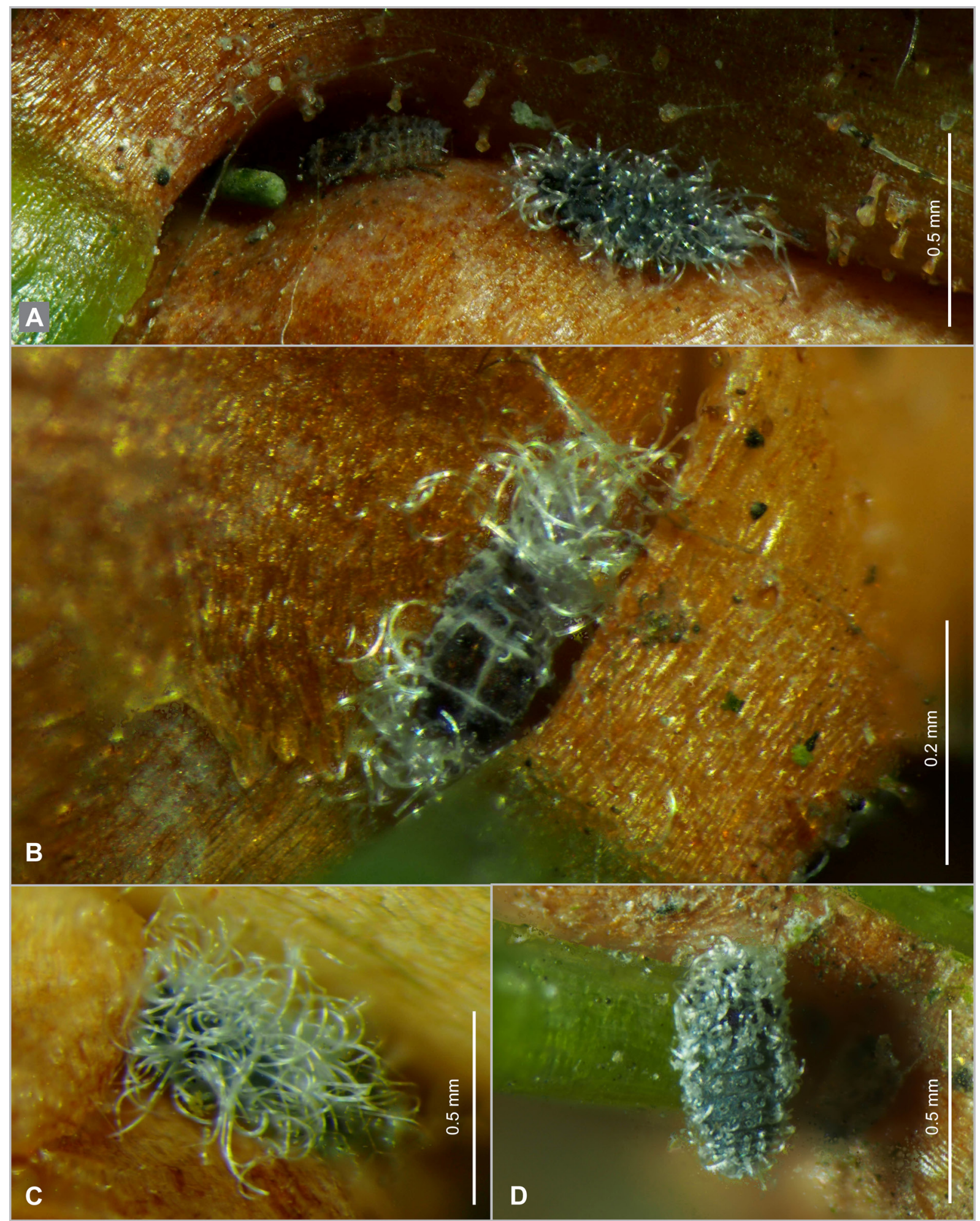

Fig. 20. Adelges (Sacciphantes) abietis (Linnaeus, 1758) on Picea abies in winter. A. First instar exuvium and second instar nymphs. B. First instar exuvium. C-D. Second instar nymphs, in D with wax filaments worn off. 


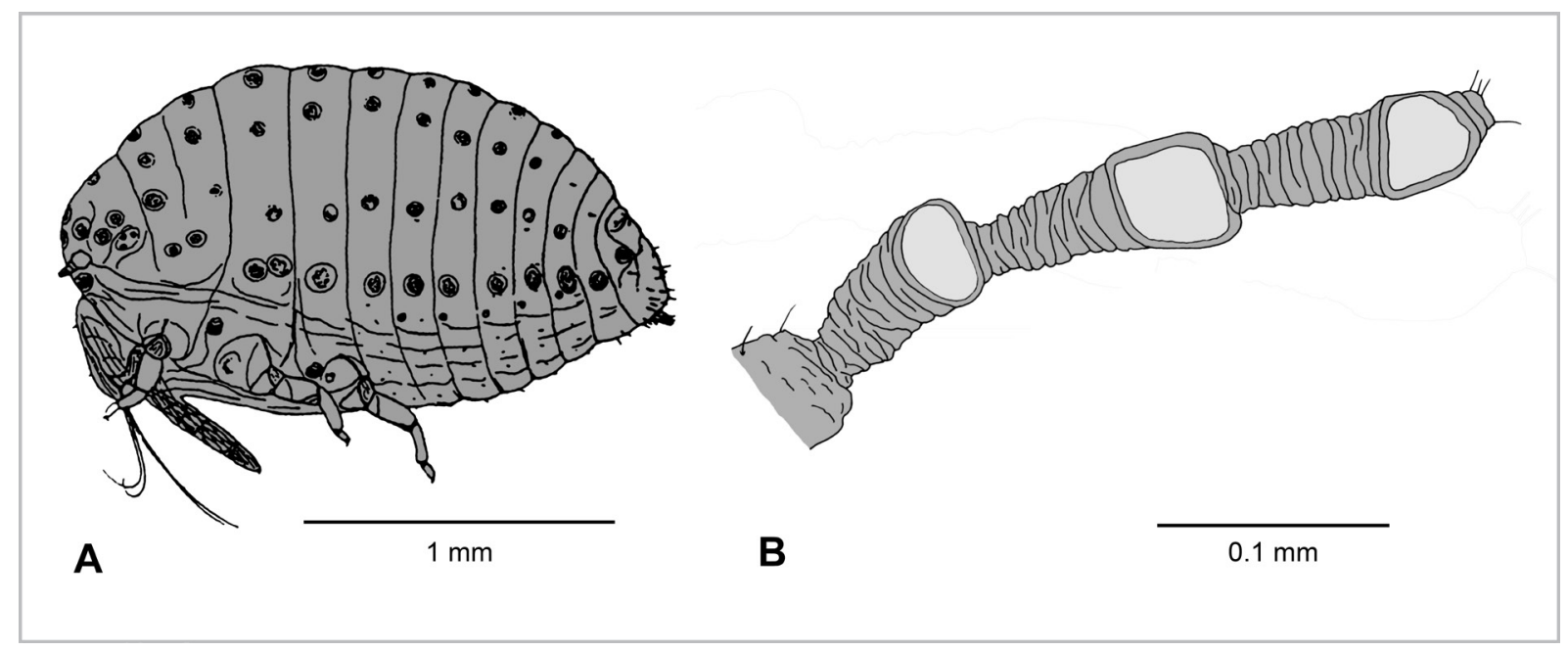

Fig. 21. Adelges (Sacciphantes) abietis (Linnaeus, 1758). A. Adult pseudofundatrix. B. Antenna of alate gallicola. (After Börner 1908, redrawn and modified.)

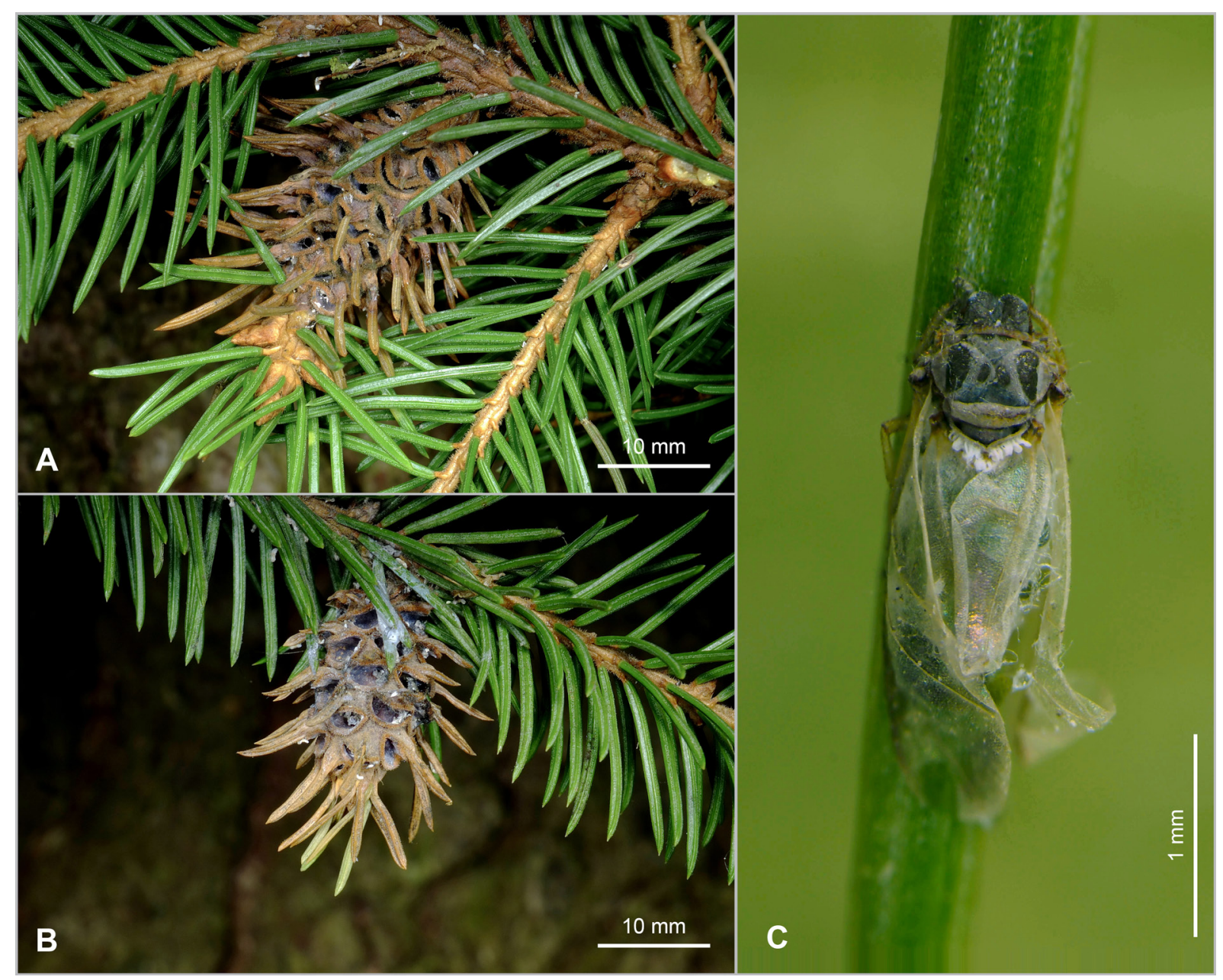

Fig. 22. Adelges (Sacciphantes) viridis (Ratzeburg, 1843). A-B. Abandoned galls on Picea abies. C. Alate gallicola (on Larix decidua). 


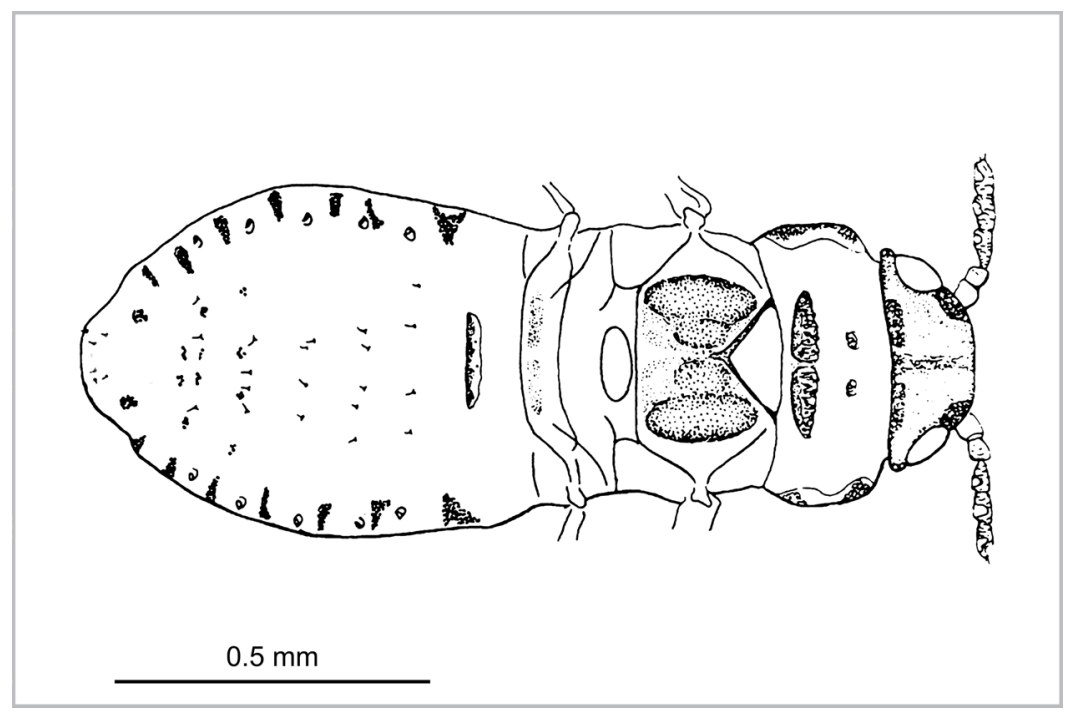

Fig. 23. Adelges (Sacciphantes) viridis (Ratzeburg, 1843), sexupara (after Carter 1971, redrawn and modified from Heie 2004).

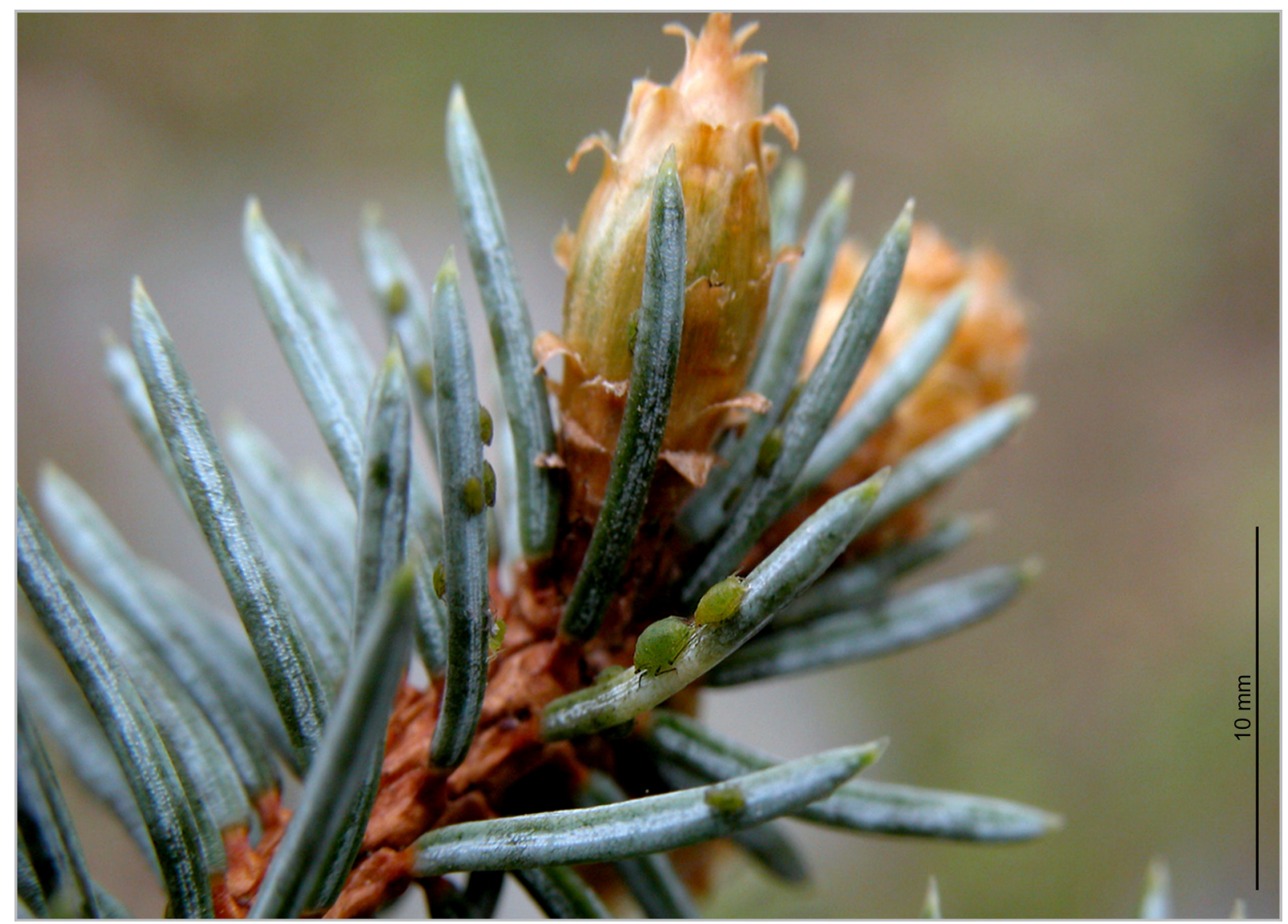

Fig. 24. Elatobium abietinum (Walker, 1849). Colony on needles of Picea pungens. 


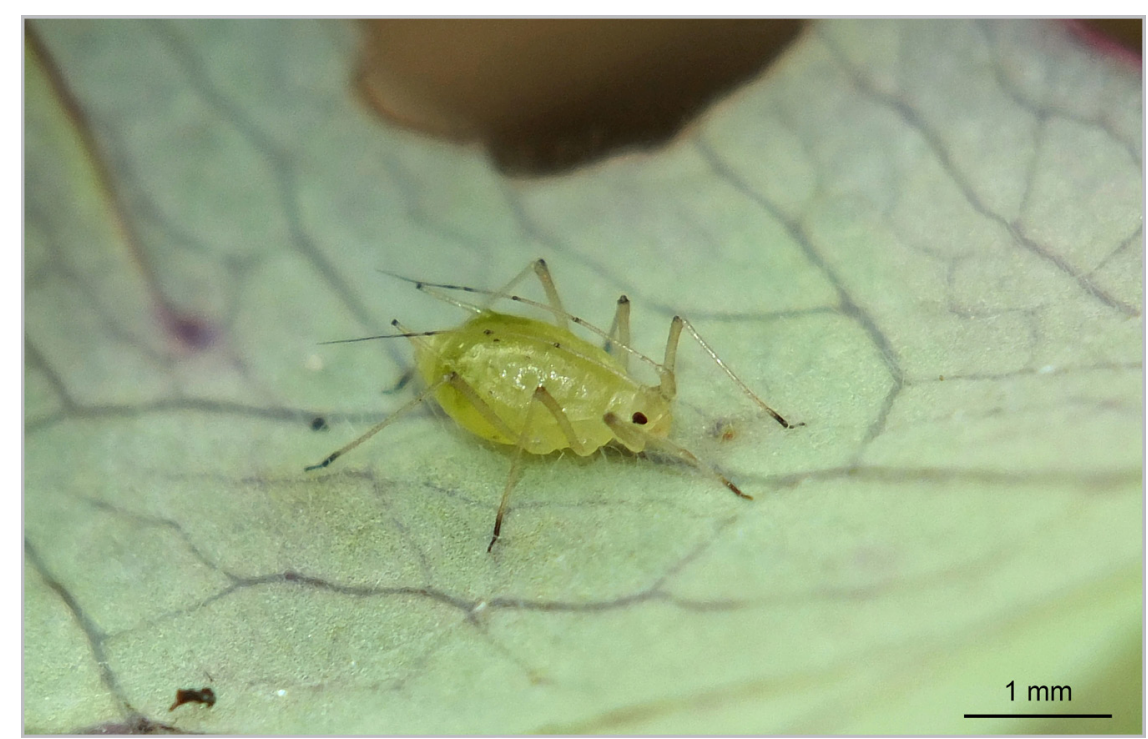

Fig. 25. Aulacorthum (Aulacorthum) solani (Kaltenbach, 1843). Aptera on Aquilegia vulgaris L. (Ranunculaceae).

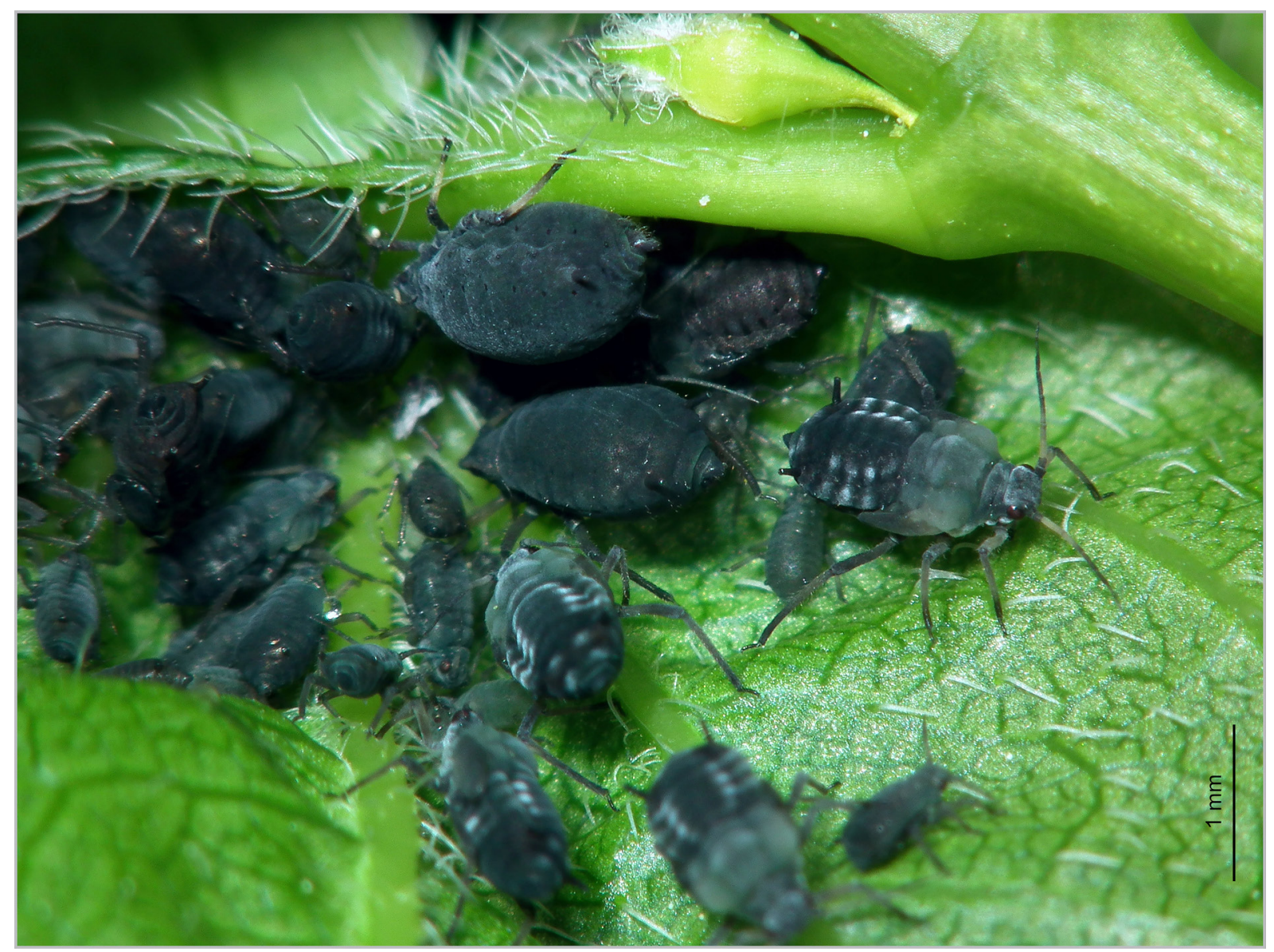

Fig. 26. Aphis (Aphis) fabae Scopoli, 1763. Colony on Philadelphus coronarius L. (Hydrangeaceae). 


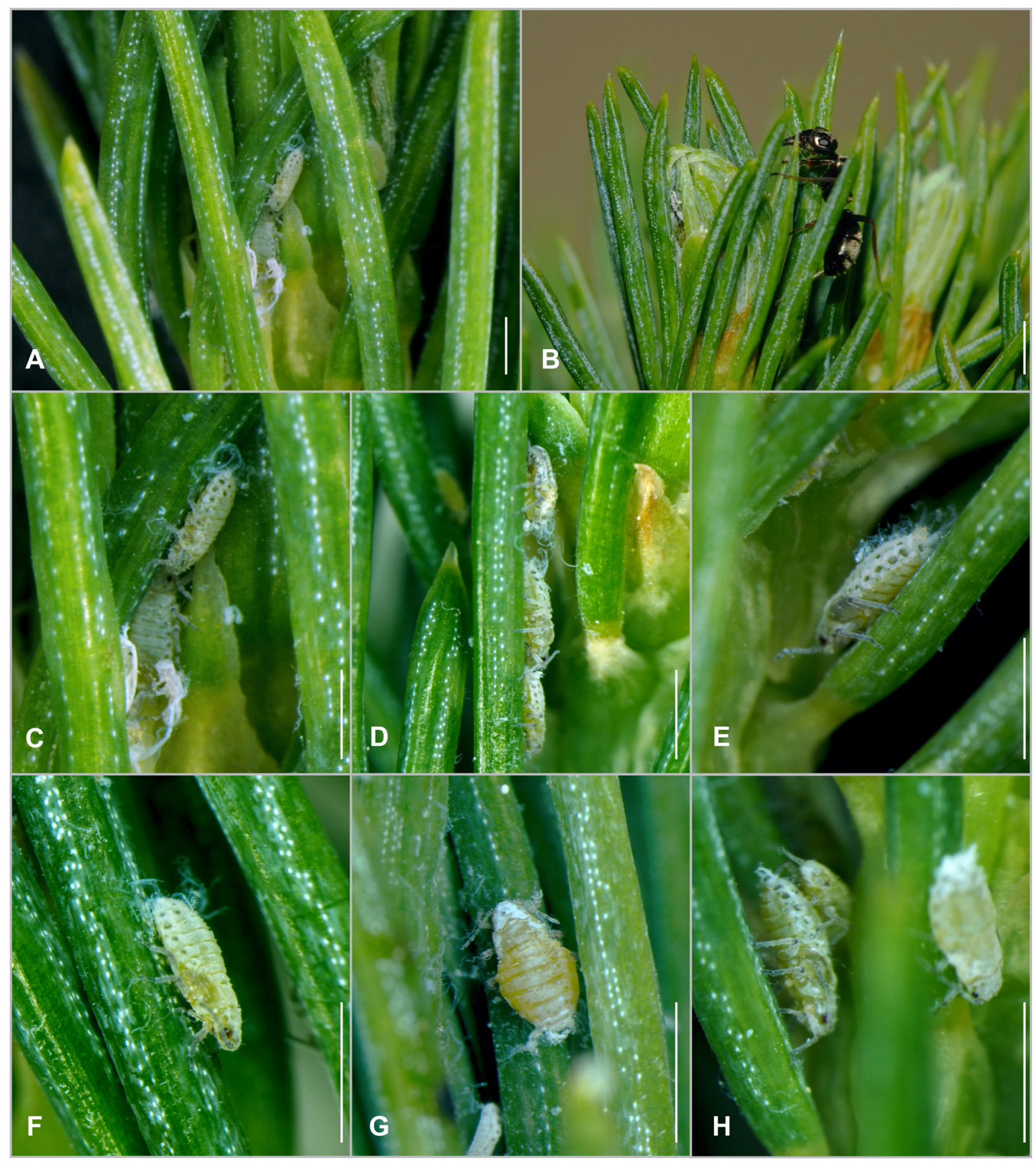

Fig. 27. Mindarus obliquus (Cholodkovsky, 1896), small colonies on Picea glauca. Apterae and young alatae (with wing pads). The ant in B is Lasius niger (Linnaeus, 1758). Scale bars: $1 \mathrm{~mm}$. 


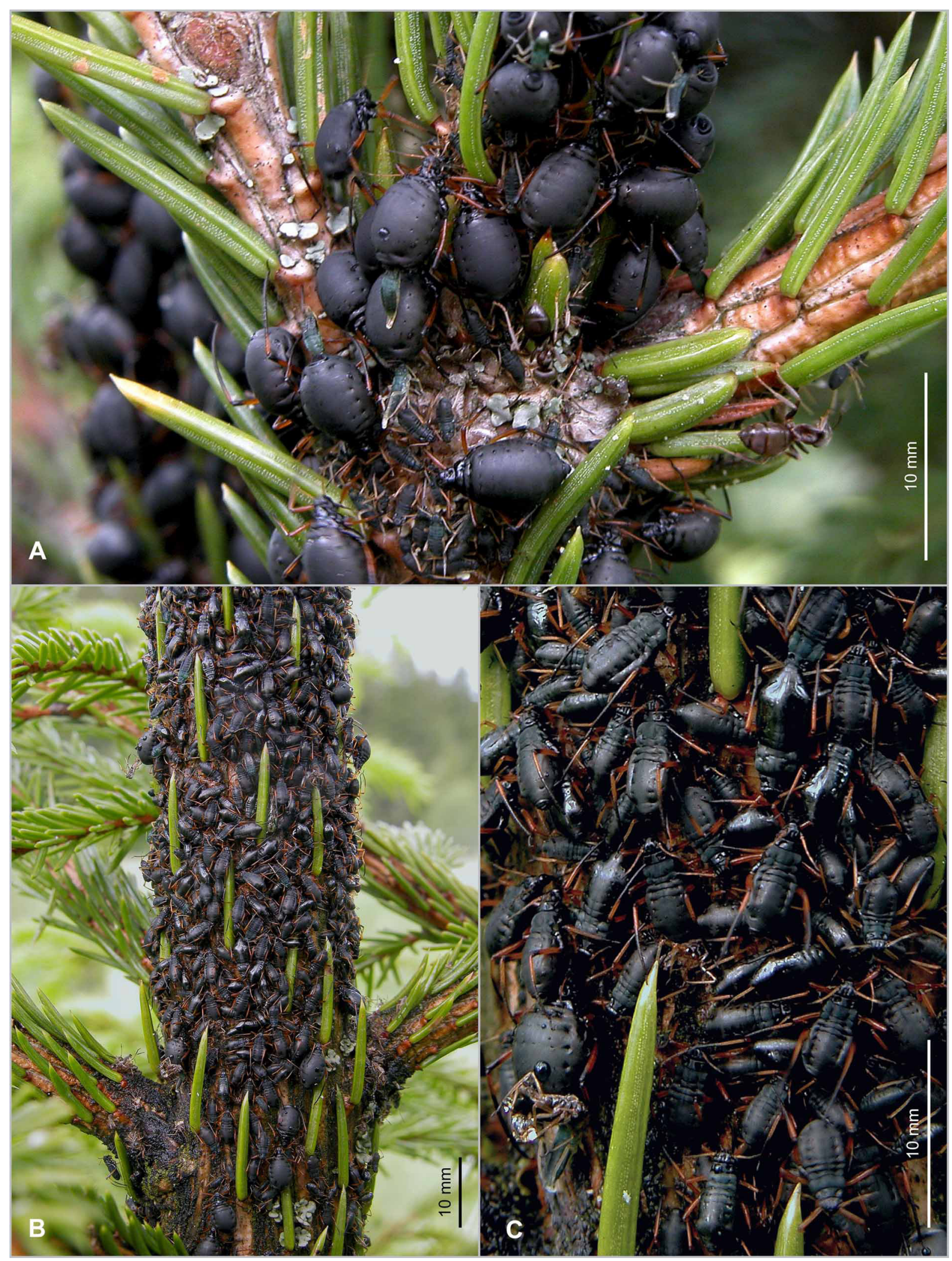

Fig. 28. Cinara (Cinara) piceae (Panzer, 1801) on Picea abies. Colonies on upper part of trunk of young tree. A. Apterae with small nymphs, attended by Lasius niger (Linnaeus, 1758). B-C. Half-grown and full-grown young alatae, and a few apterae. 


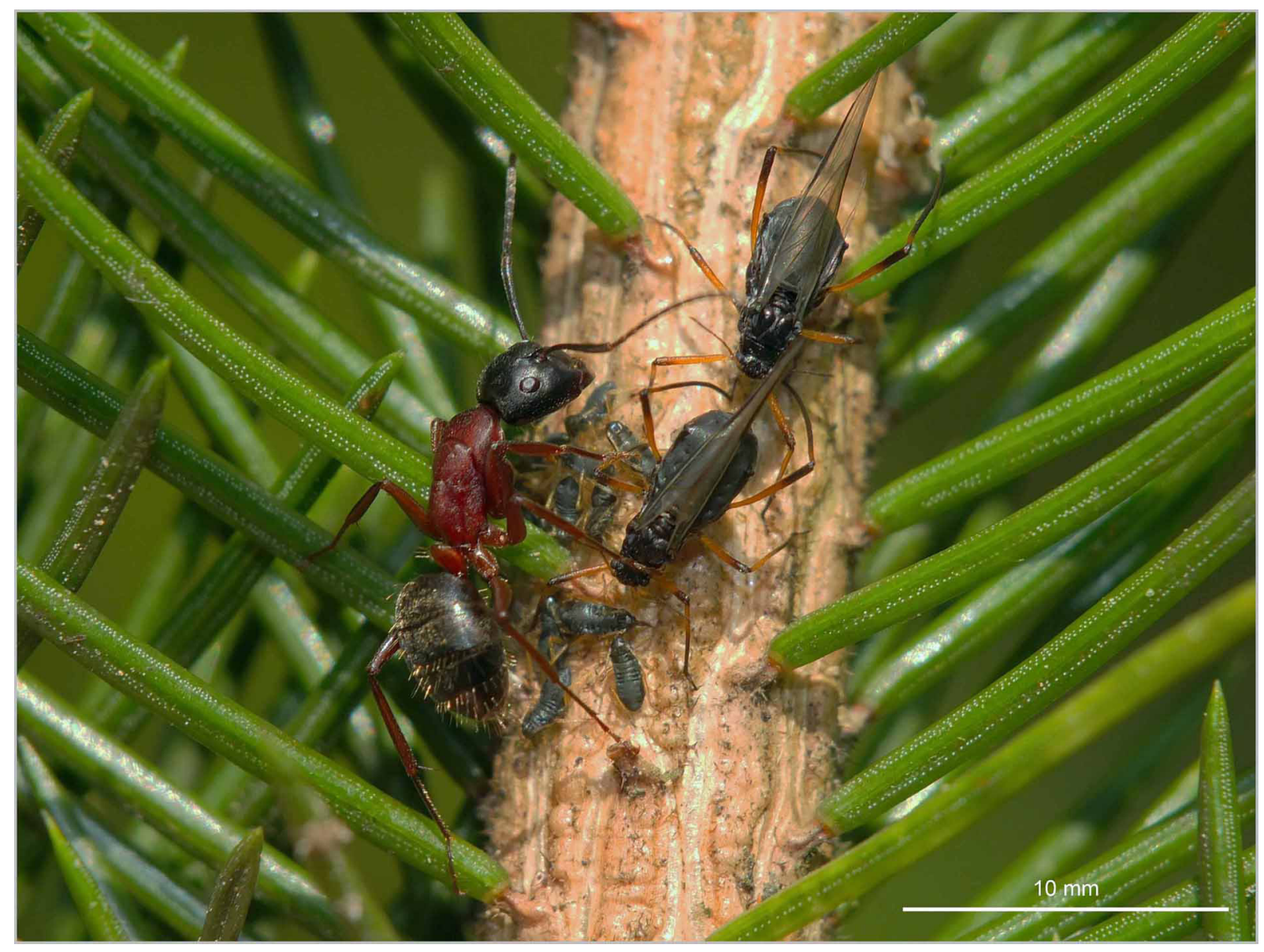

Fig. 29. Cinara (Cinara) piceae (Panzer, 1801) on Picea abies. Alatae with young nymphs, attended by Camponotus herculeanus (Linnaeus, 1758). 

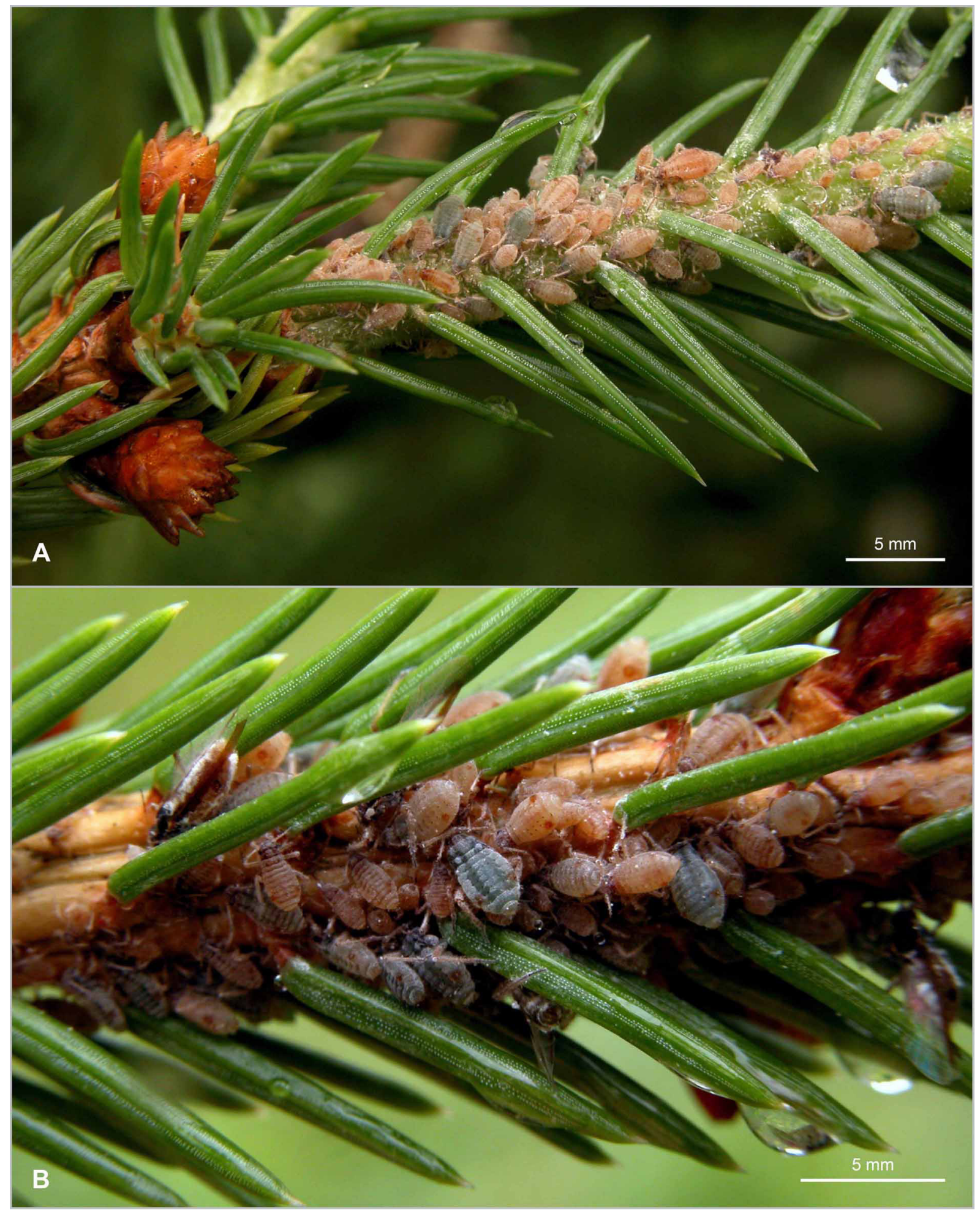

Fig. 30. Cinara (Cinara) pilicornis (Hartig, 1841) on Picea abies. Typical mid-summer colonies on branch undersides. 


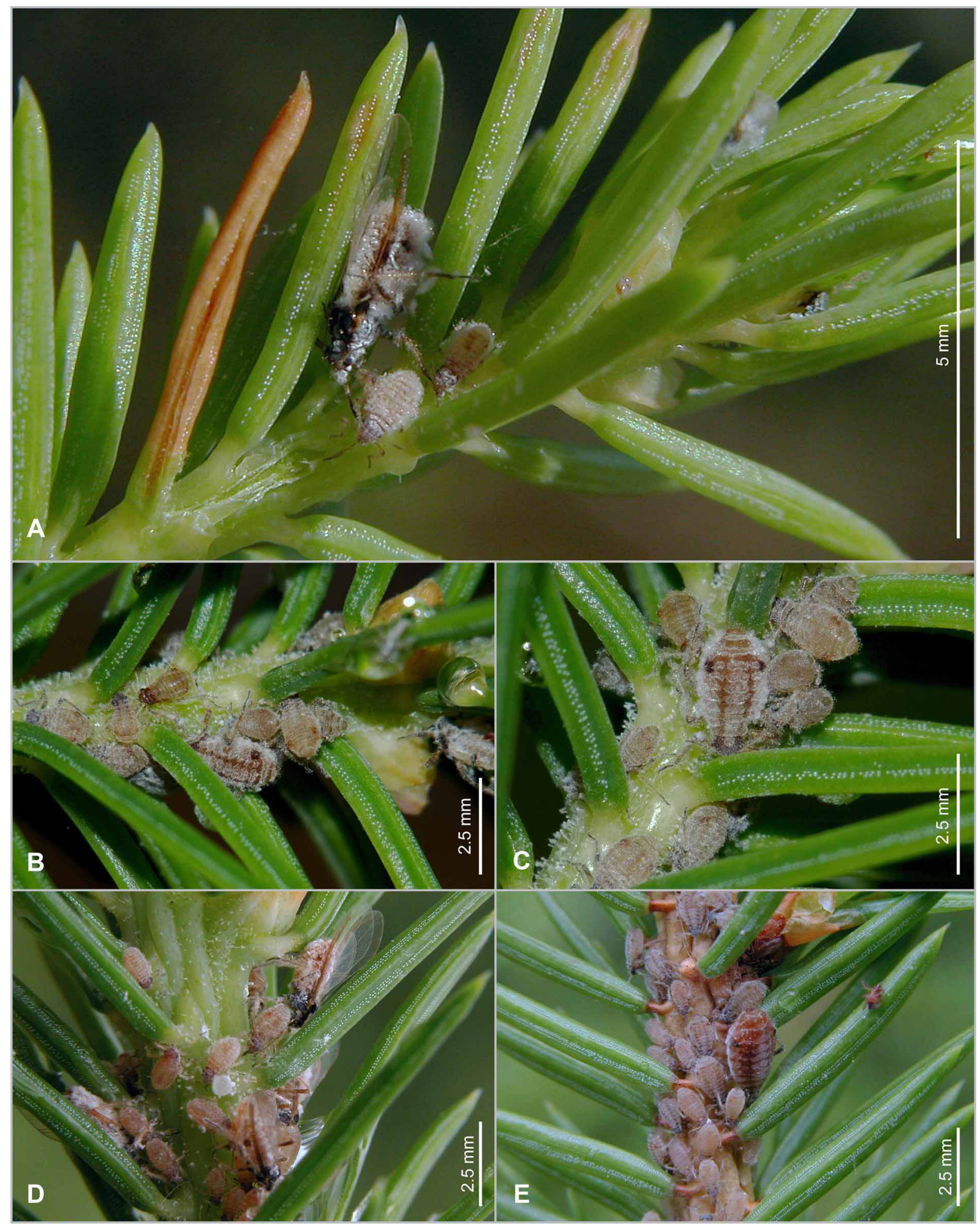

Fig. 31. Cinara (Cinara) pilicornis (Hartig, 1841). Newly founded colonies on young shoots of Picea abies. Wax cover intact and pattern very distinct. Note the thick wax wool on the alatae (A, D). 


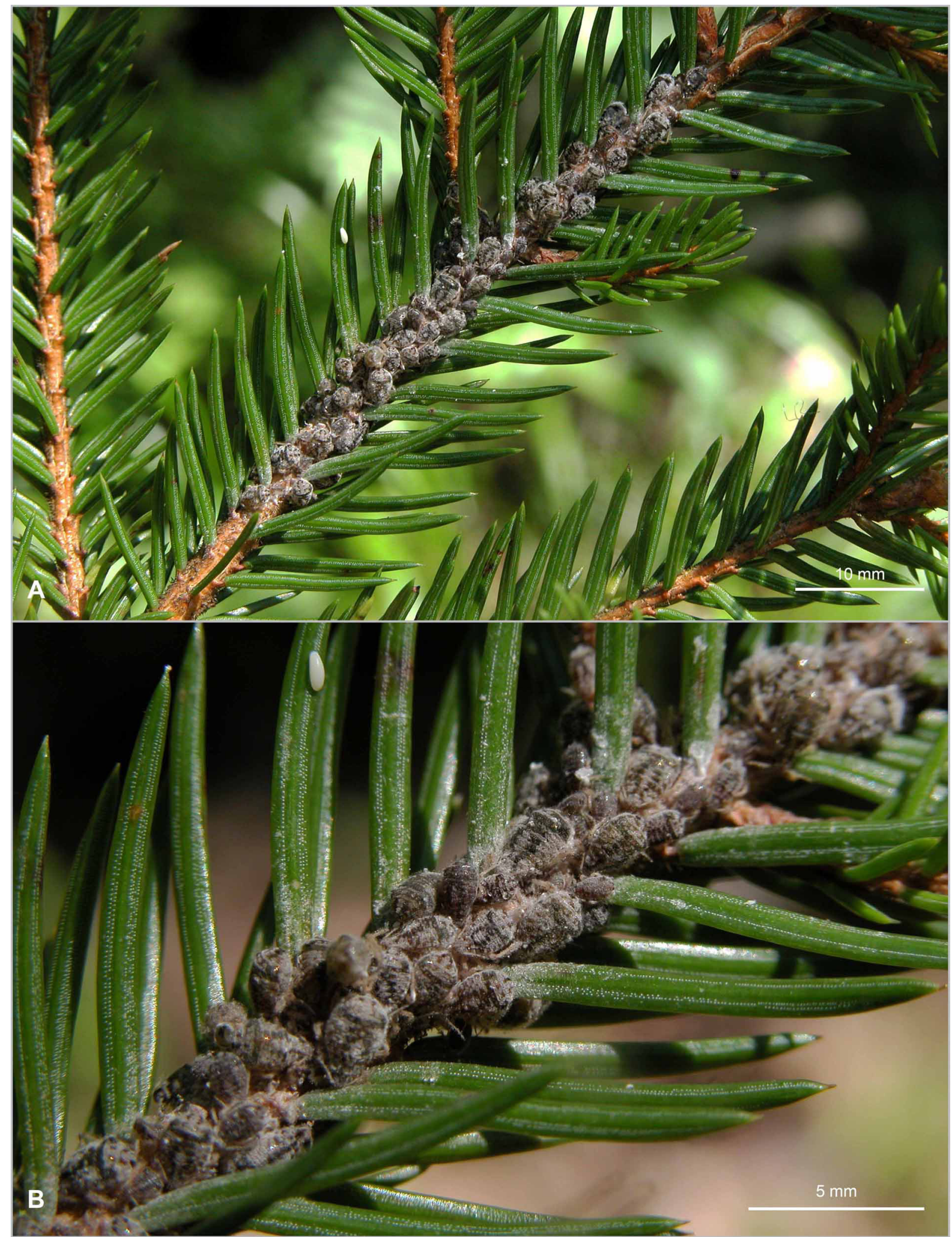

Fig. 32. Cinara (Cinara) costata (Zetterstedt, 1828). Colony on underside of a 1-2 year-old twig of Picea abies. 


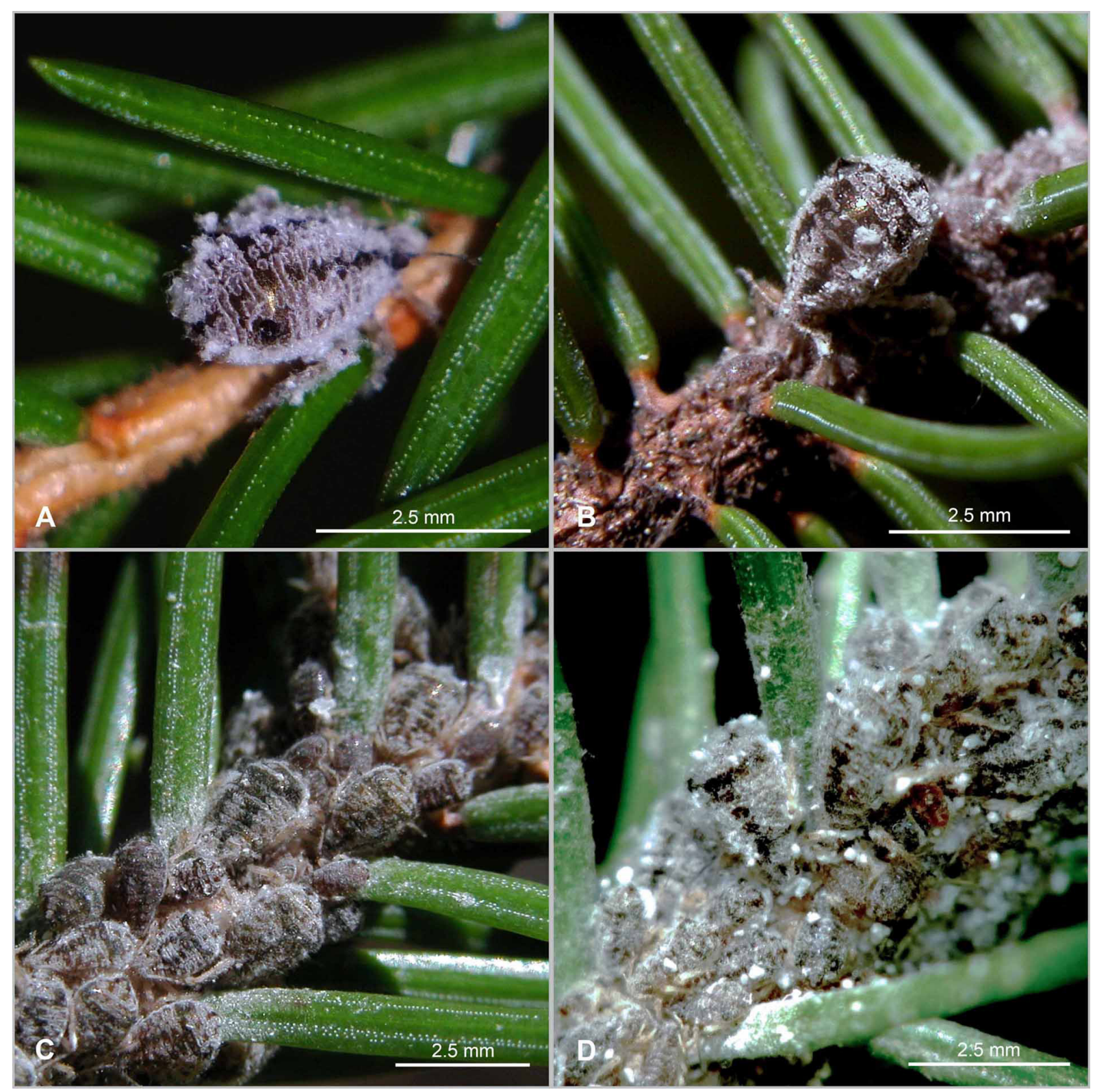

Fig. 33. Cinara (Cinara) costata (Zetterstedt, 1828) on Picea abies, showing different stages of development and deterioration of wax pattern. 


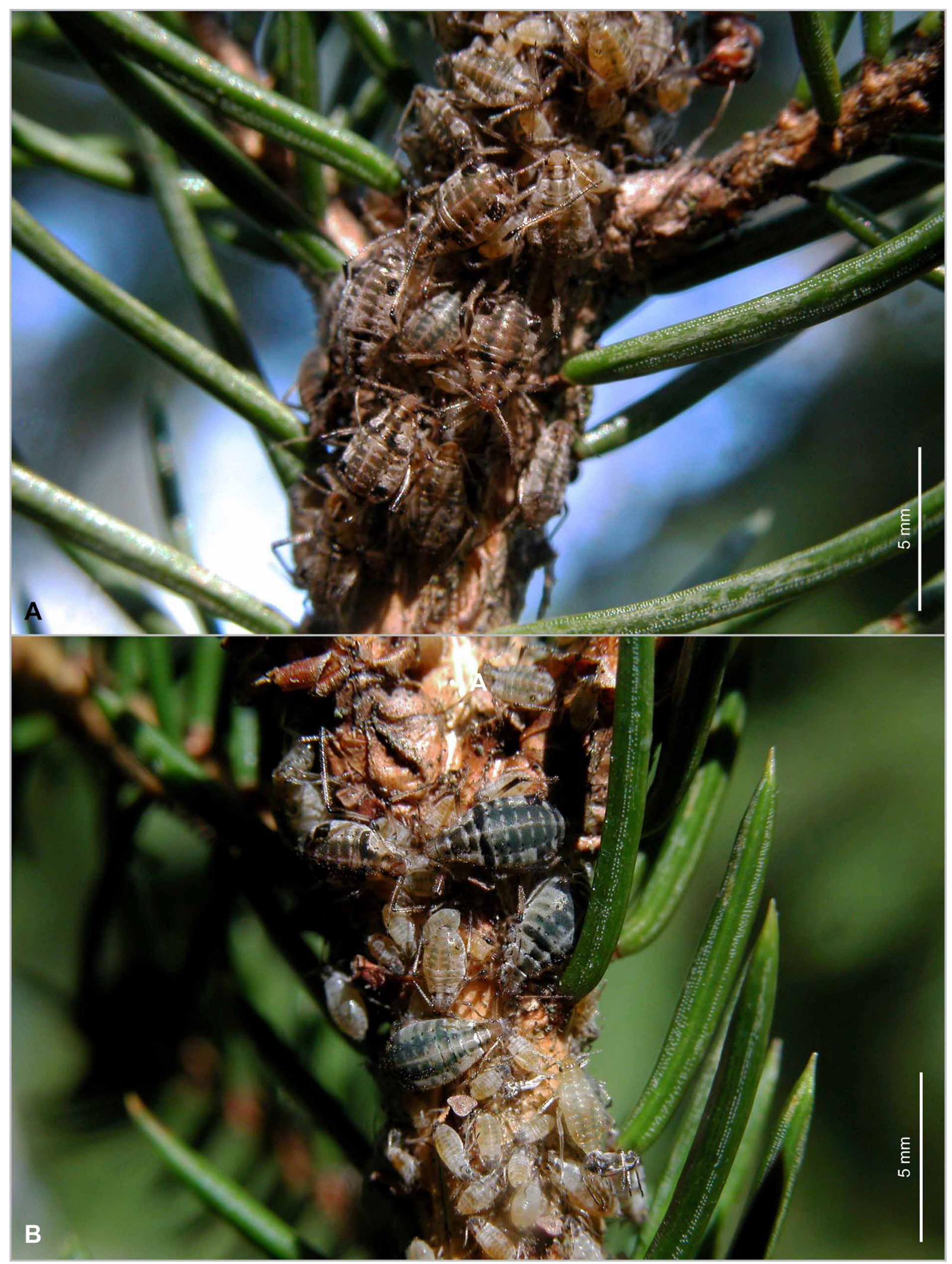

Fig. 34. Cinara (Cinara) pruinosa (Hartig, 1841). Colony on underside of branch of Picea abies, attended by Formica aquilonia Yarrow, 1955. Adult and juvenile apterae. 


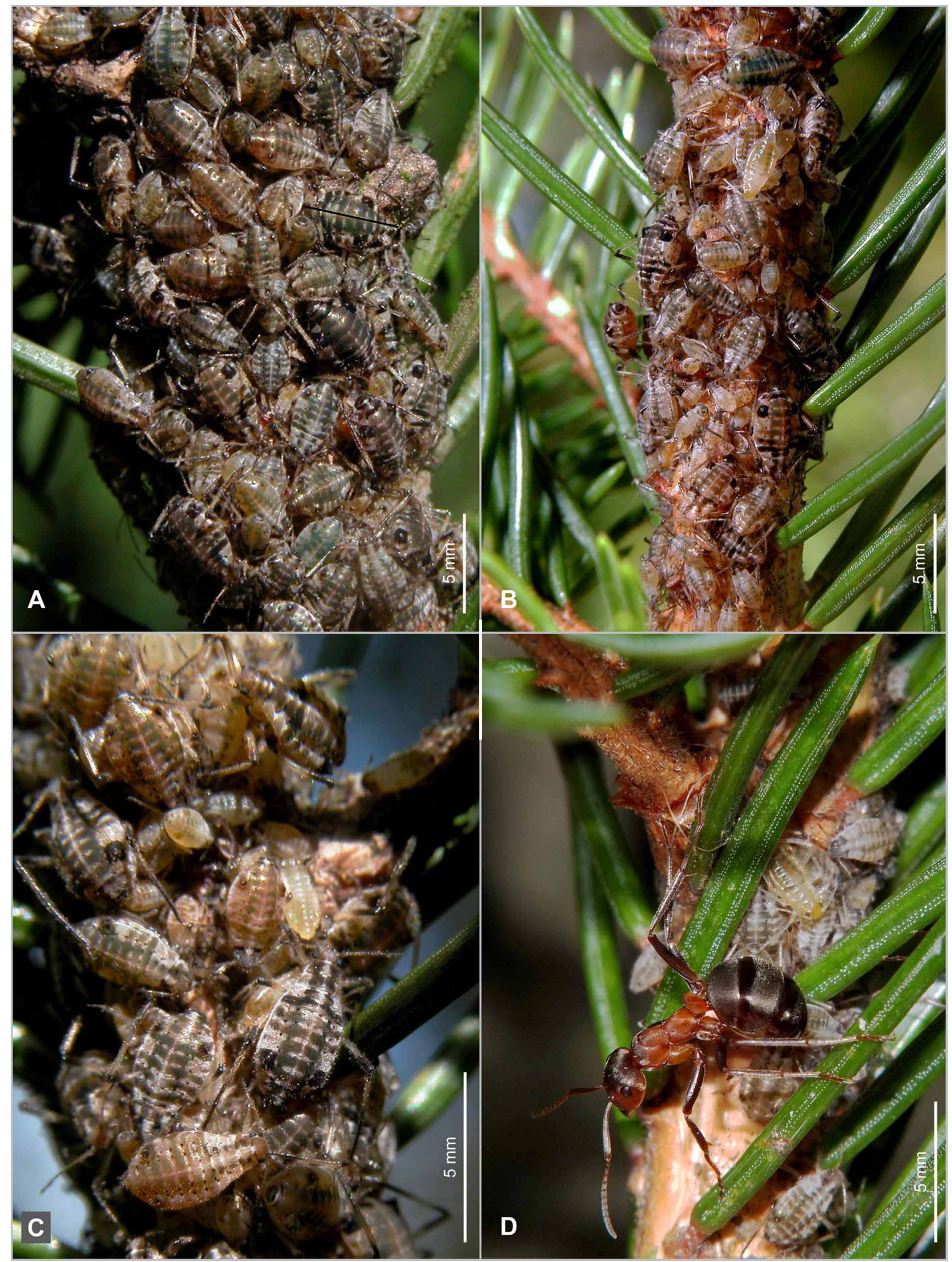

Fig. 35. Cinara (Cinara) pruinosa (Hartig, 1841). Colony on underside of branch of Picea abies, attended by Formica aquilonia Yarrow, 1955 (D). Adult apterae, juvenile apterae and alatae. 

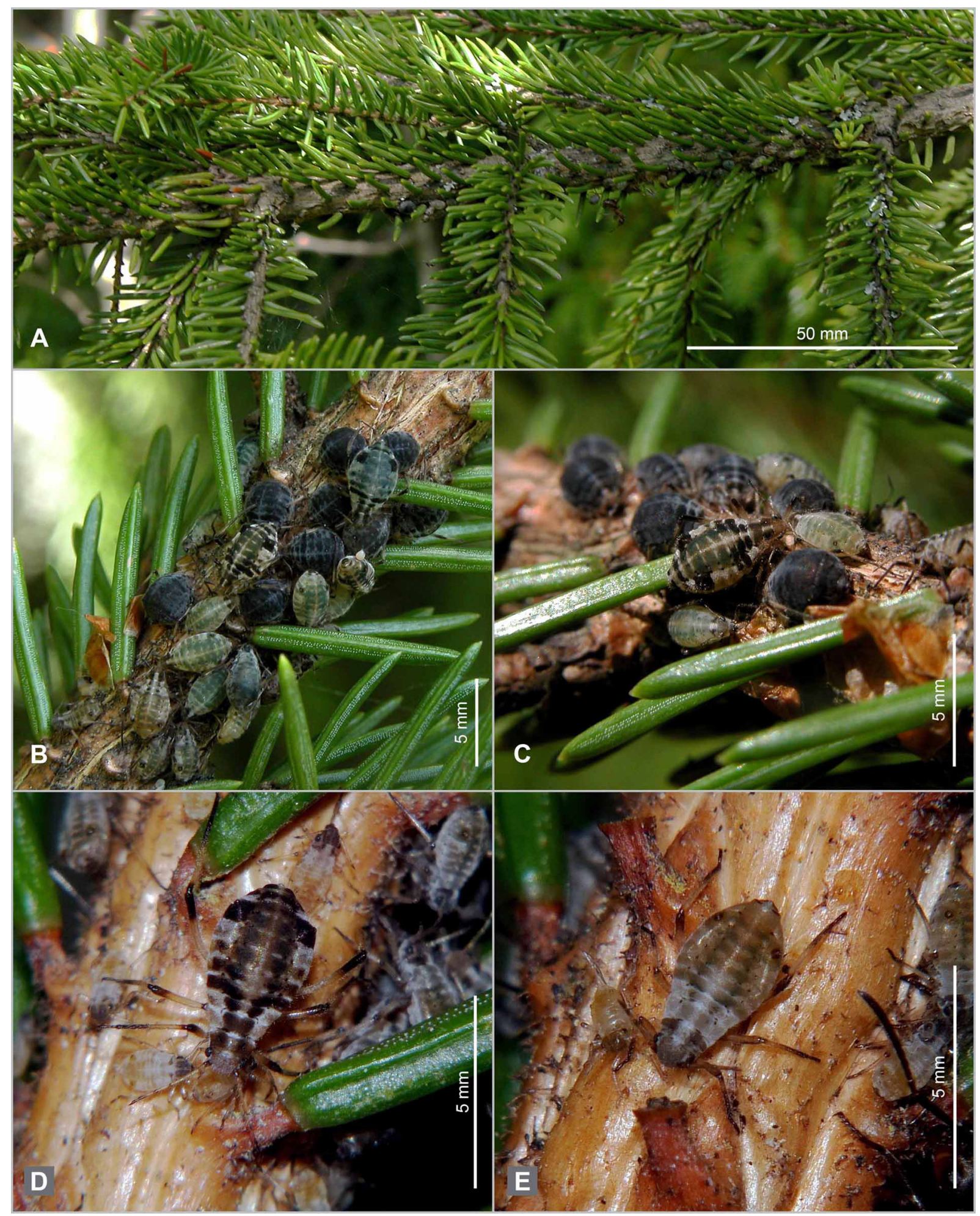

Fig. 36. Cinara (Cinara) pruinosa (Hartig, 1841) on Picea abies. A. Branch with colony and attendant Formica aquilonia Yarrow, 1955 on underside. B-C. Adult and juvenile apterae. The dark individuals are parasitized. D. Adult aptera. E. Full-grown juvenile aptera. 


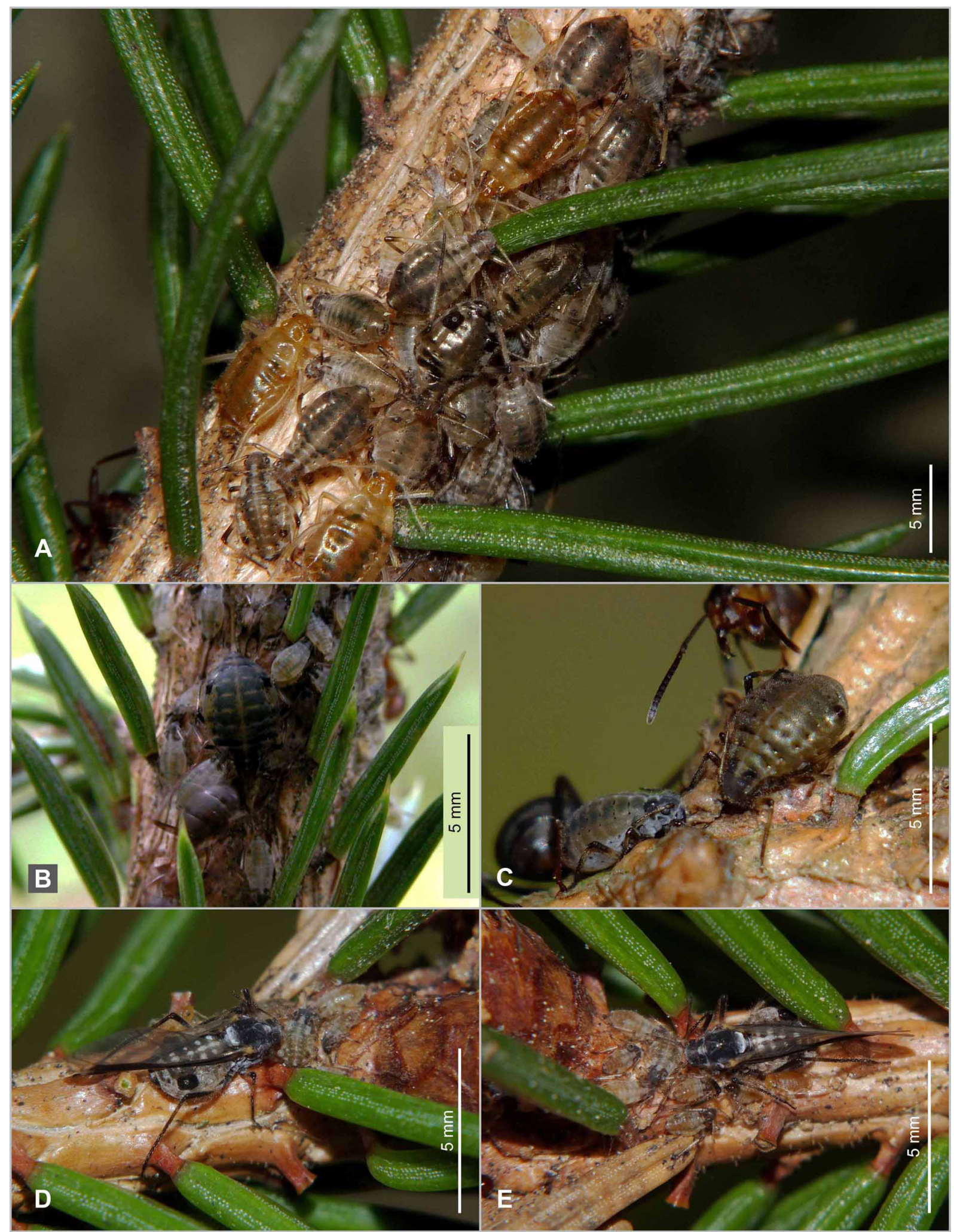

Fig. 37. Cinara (Cinara) pruinosa (Hartig, 1841) on Picea abies. A. Adult and juvenile apterae, including teneral adults. B. Fundatrix with juvenile apterae. C. Juvenile and adult fundatrix, attended by Formica aquilonia Yarrow, 1955. D-E. Alatae with newly founded colonies. 


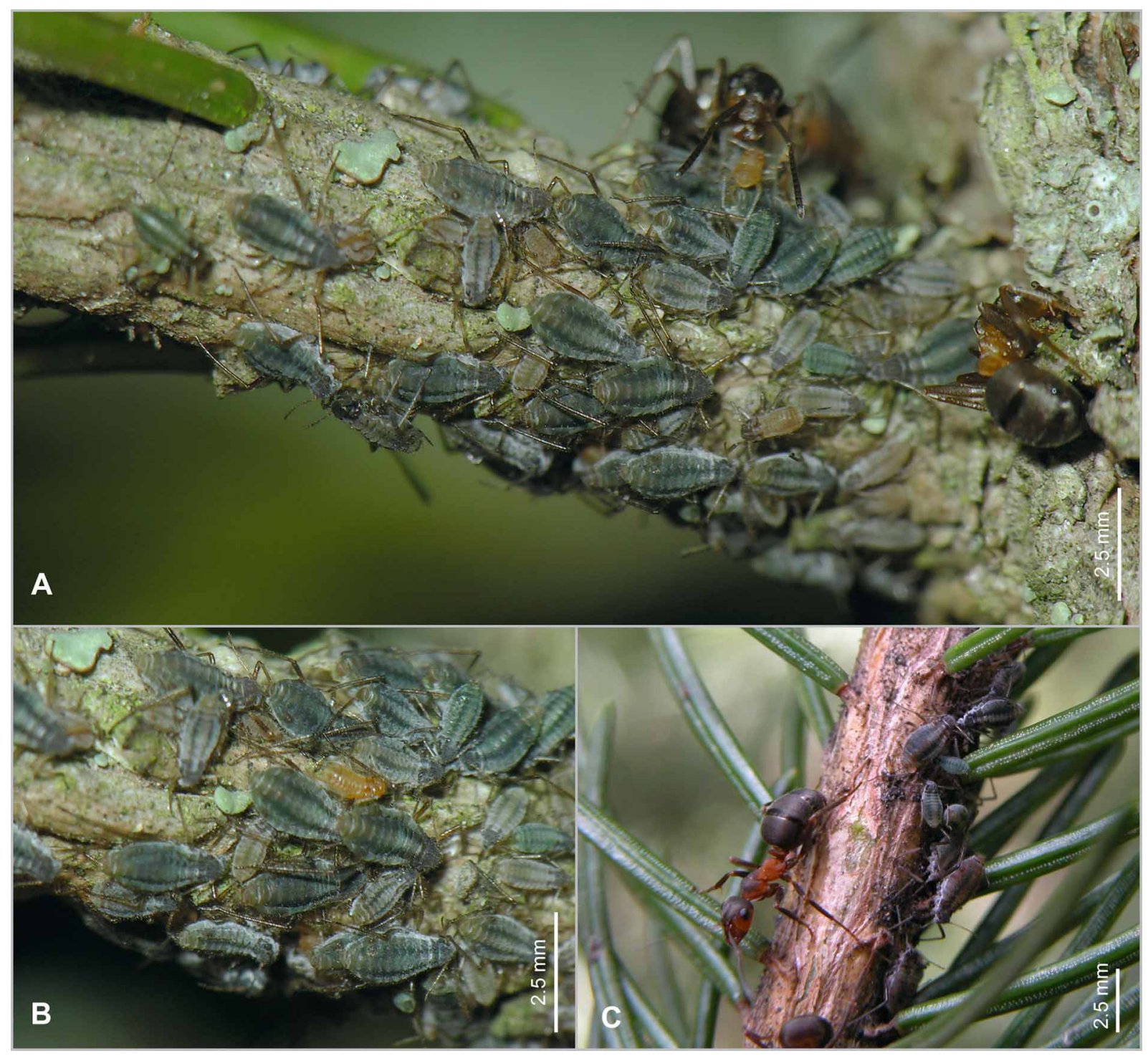

Fig. 38. Cinara (Cinara) piceicola (Cholodkovsky, 1896). Apterae and juveniles on branches of Picea abies, attended by Formica aquilonia Yarrow, 1955. 


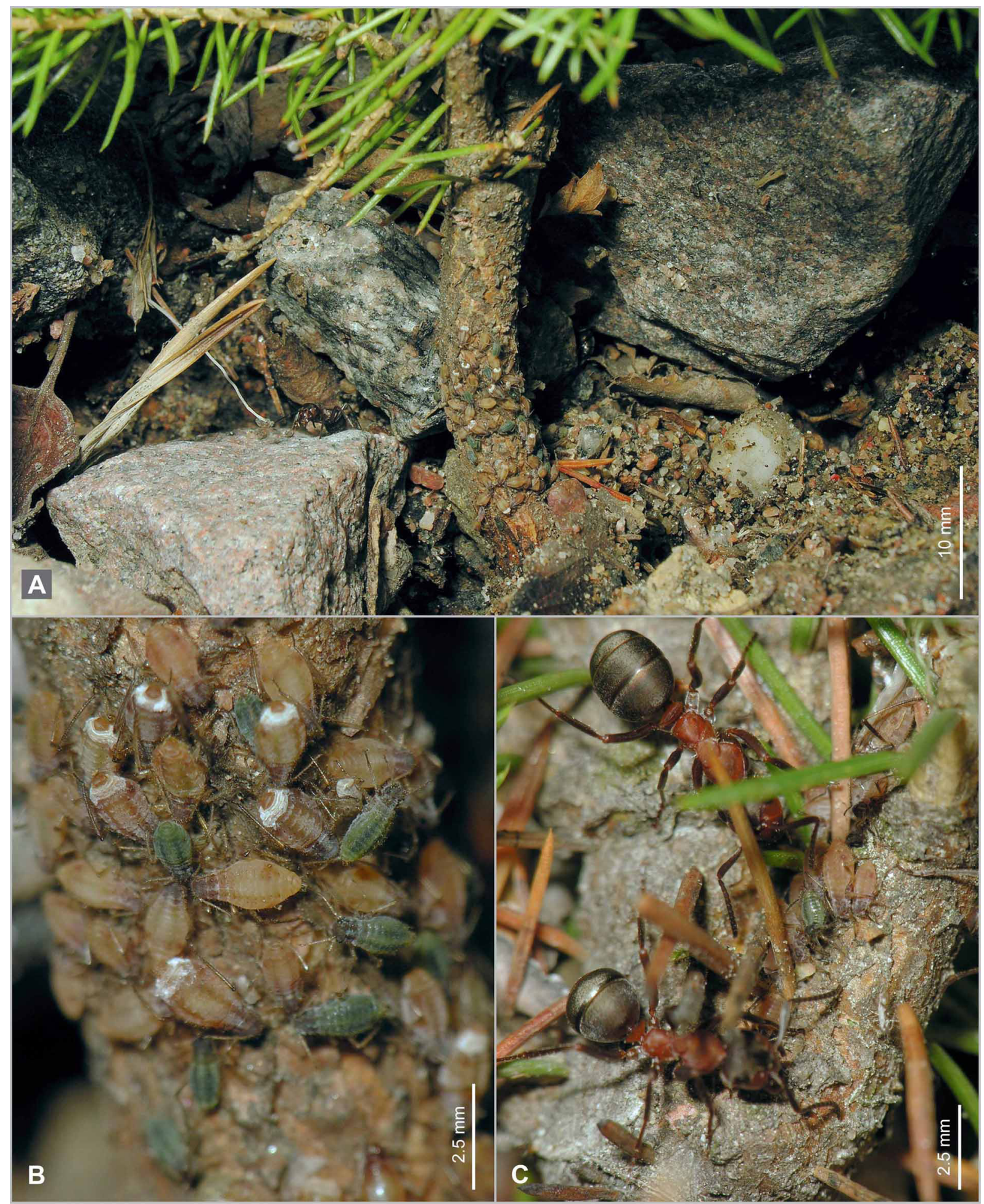

Fig. 39. Cinara (Cinara) piceicola (Cholodkovsky, 1896) on dwarfish Picea abies. A-B. Colony of apterae and oviparae among gravel at ground level. C. Apterae on stem, attended by Formica lugubris Zetterstedt, 1838. 


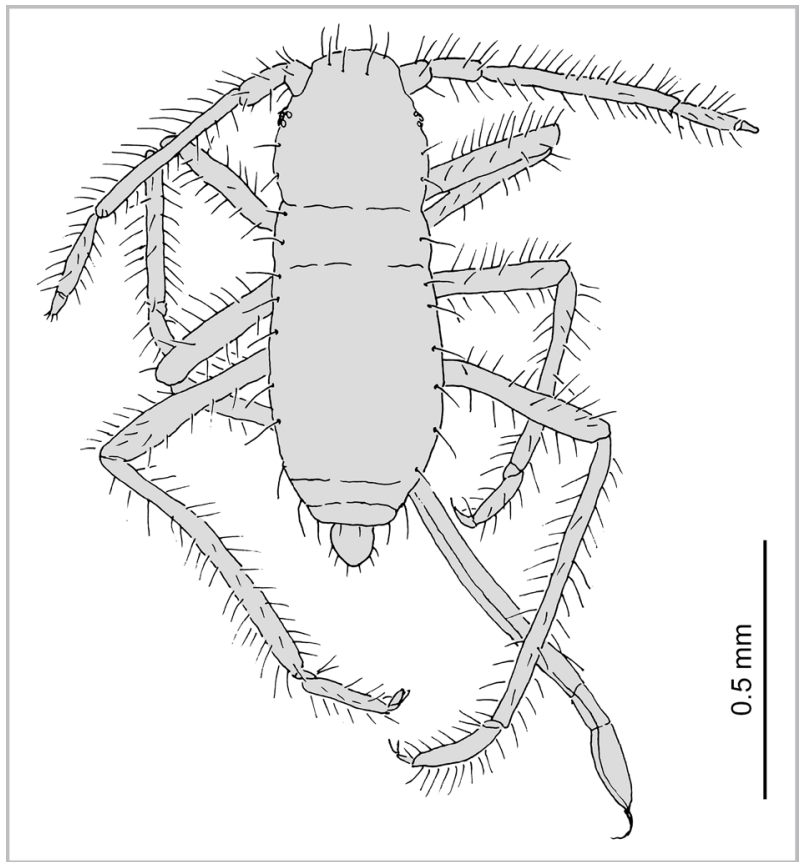

Fig. 40. Prociphilus (Prociphilus) bumeliae (Schrank, 1801). Young aptera from Abies root (after Heie 2004, redrawn). 


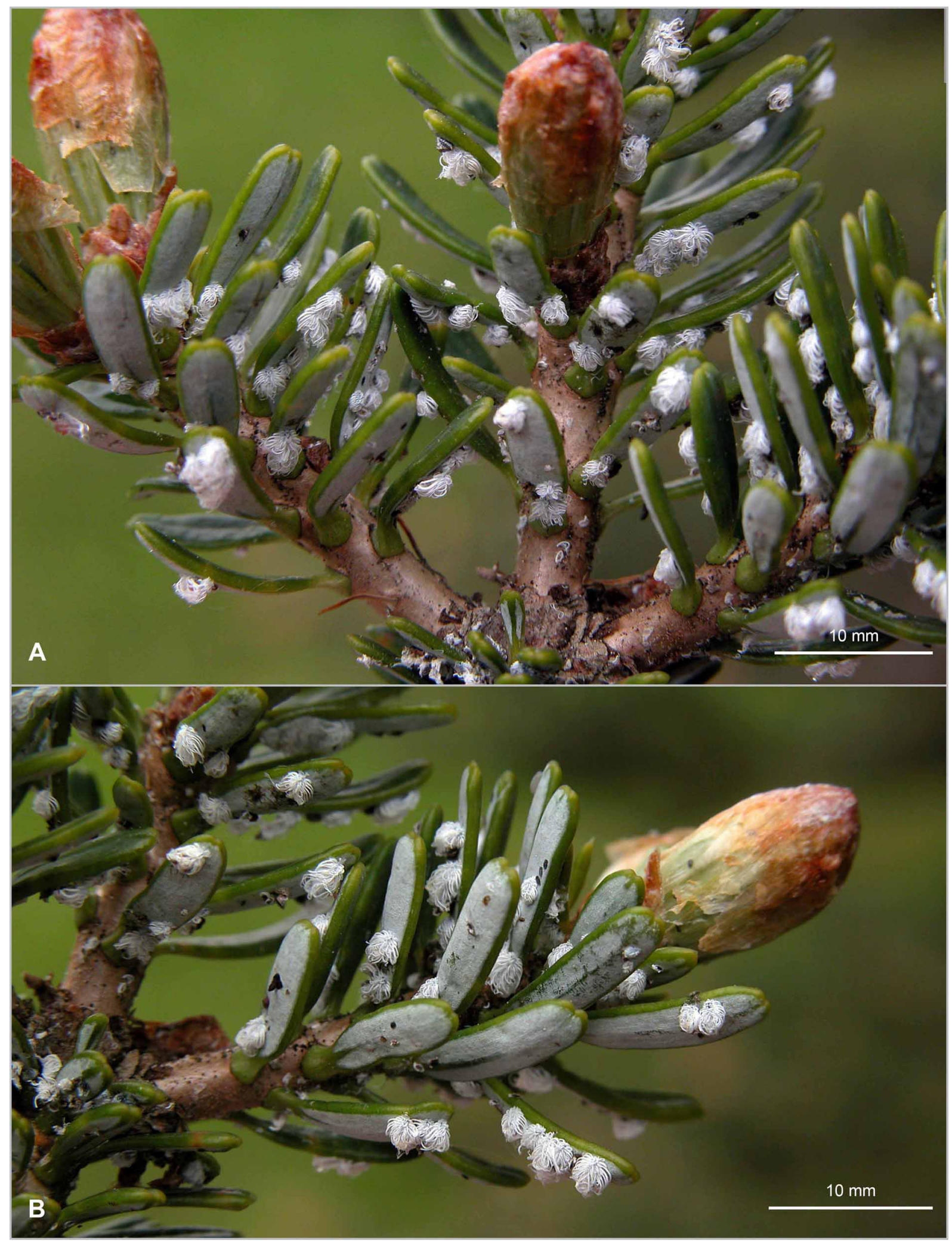

Fig. 41. Adelges (Aphrastasia) pectinatae (Cholodkovsky, 1888). Apterous progredientes on undersides of needles of Abies koreana. Note the conspicuous coarse wax filaments. 


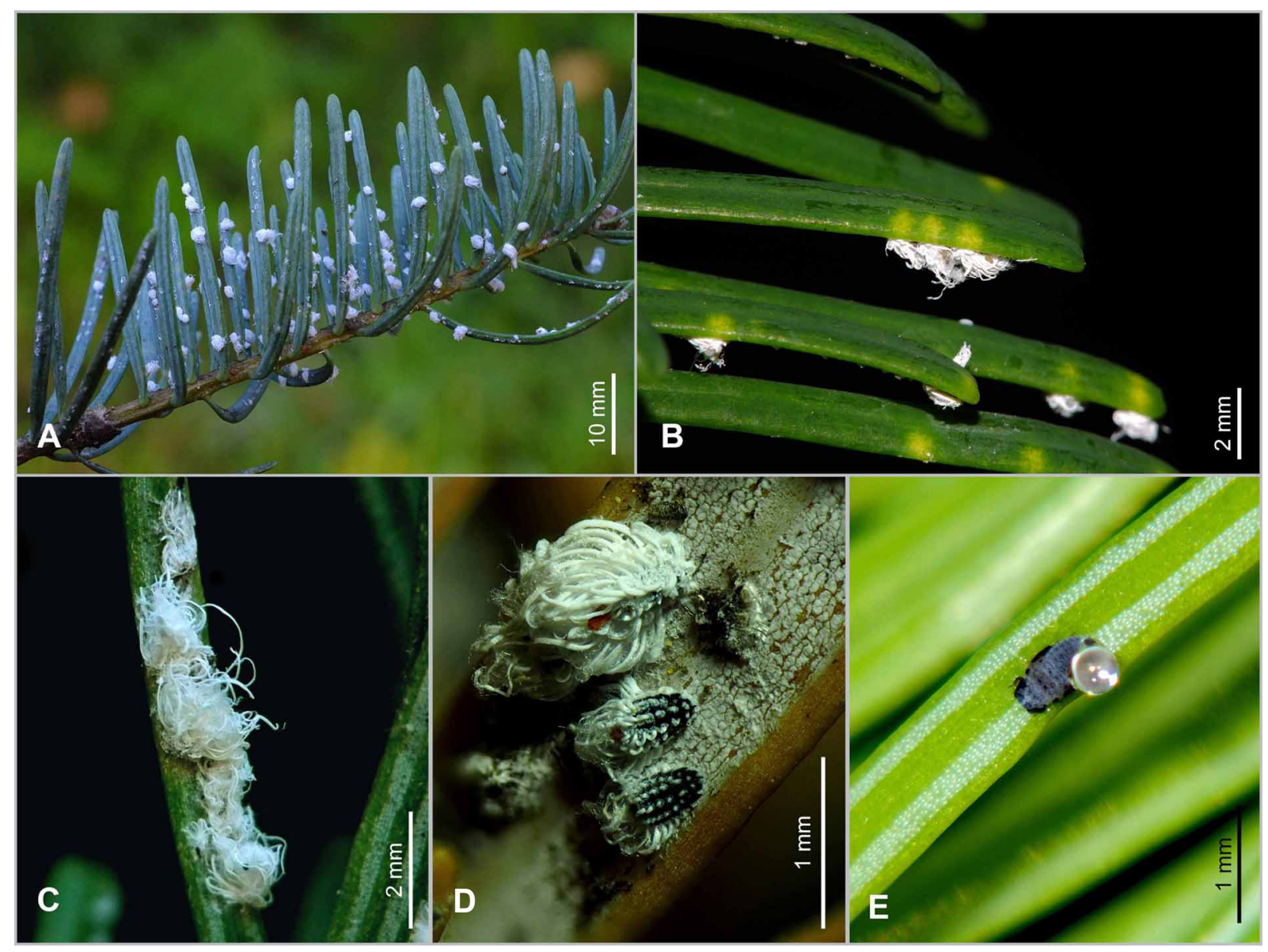

Fig. 42. Adelges (Aphrastasia) pectinatae (Cholodkovsky, 1888). A. Apterae (and neosistentes) on Abies concolor. B-C. Apterae with eggs on A. alba and Abies sp. D. Adult and juvenile apterae on A. koreana (freeze-dried sample). E. Juvenile alata on A. sibirica. 

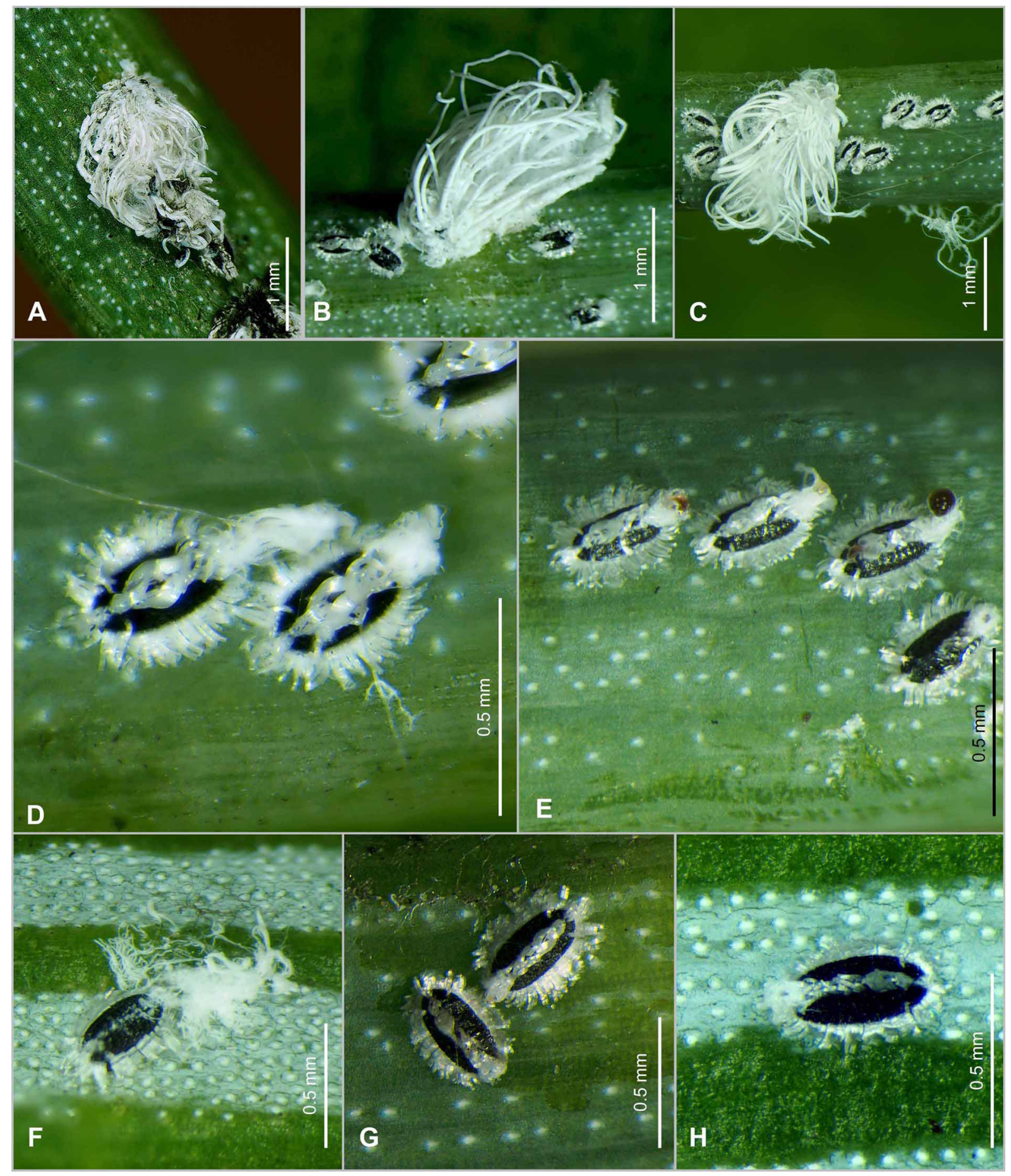

Fig. 43. Adelges (Aphrastasia) pectinatae (Cholodkovsky, 1888) on needles of Abies concolor. A-C. Apterae with first instar hibernating nymphs (neosistens). D-H. Neosistens. 


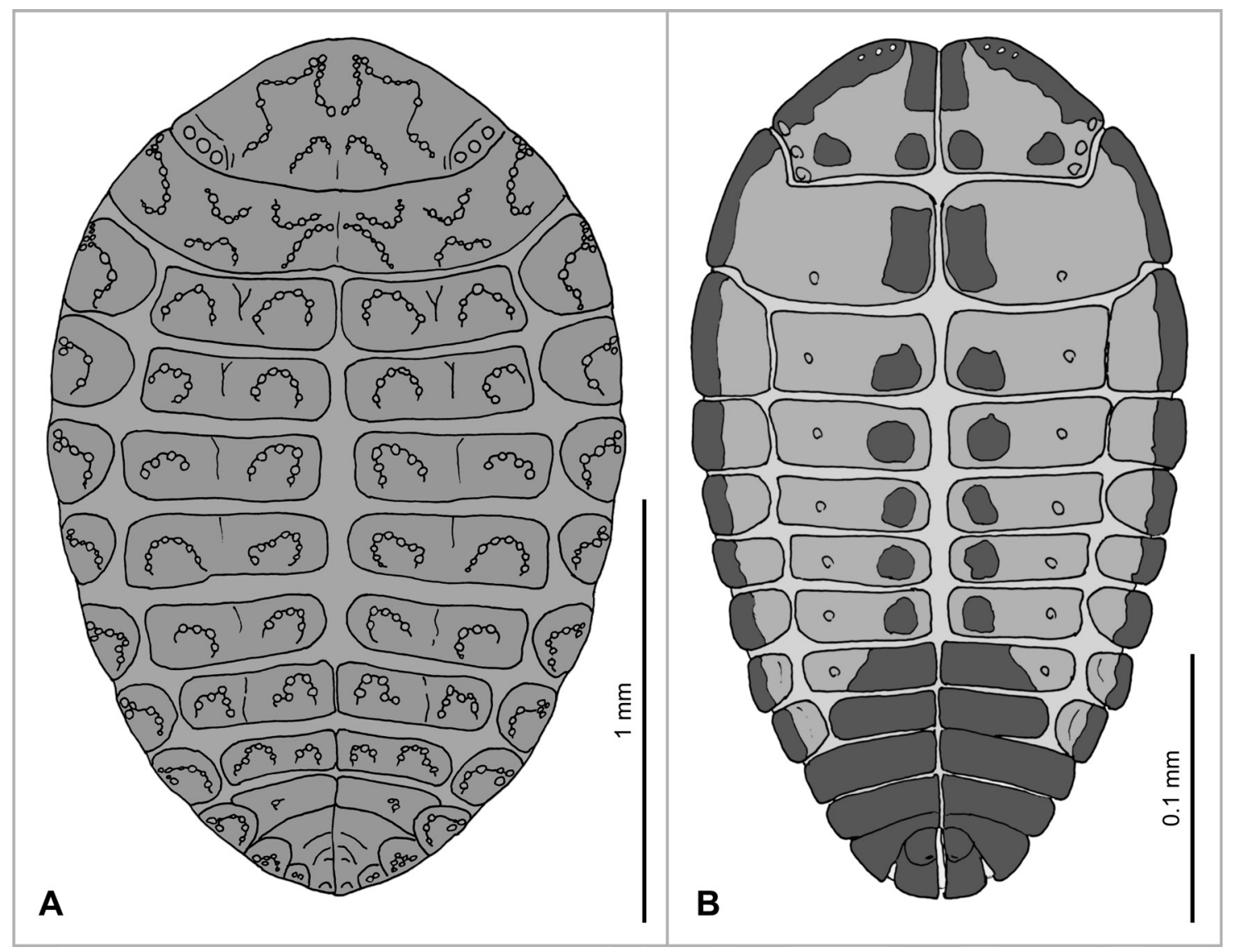

Fig. 44. Adelges (Aphrastasia) pectinatae (Cholodkovsky, 1888). A. Adult progrediens, showing arrangement of plates and wax gland pits (after Inouye 1953, modified). Note the wax gland pattern, giving rise to 'half-pipe'-shaped wax filaments. B. Hibernating first instar nymph (neosistens), with wax-exuding areas shaded (after Inouye 1953, modified and redrawn from Pashchenko 1988). 


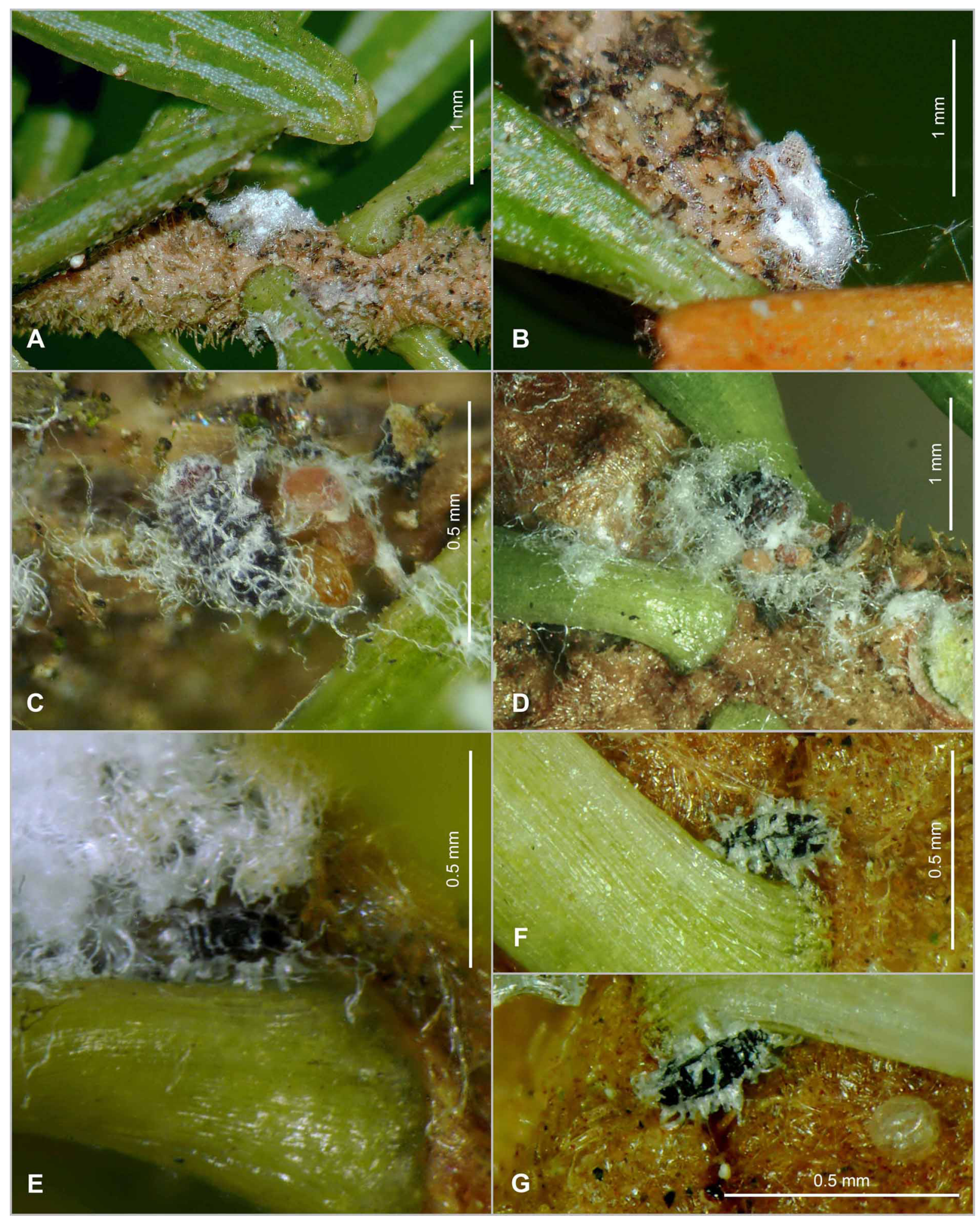

Fig. 45. Adelges (Dreyfusia) nordmannianae (Eckstein, 1890) on Abies alba. A-D. Apterous progredientes with eggs. Note the thin, woolly wax filaments. E-G. First instar hibernating nymphs (neosistens). 


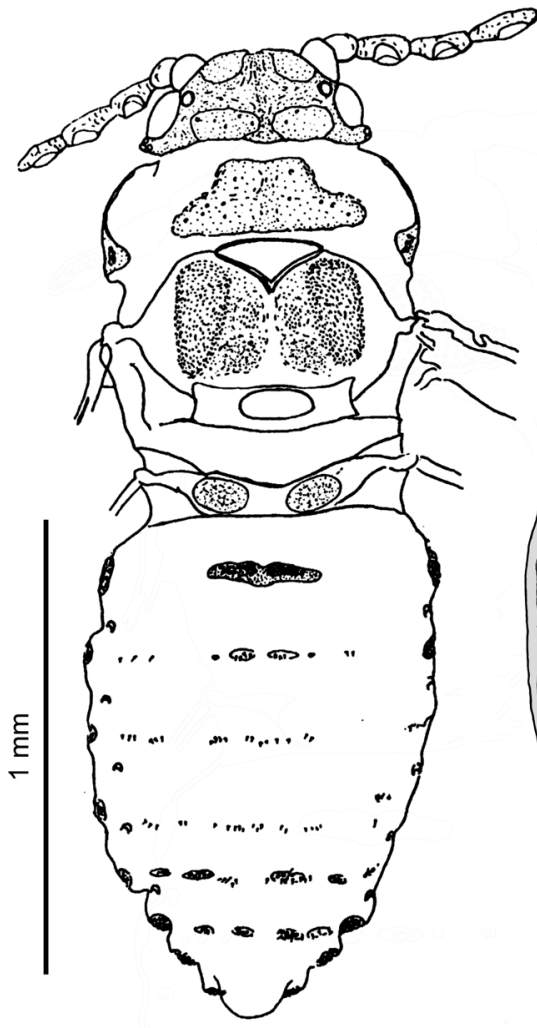

A

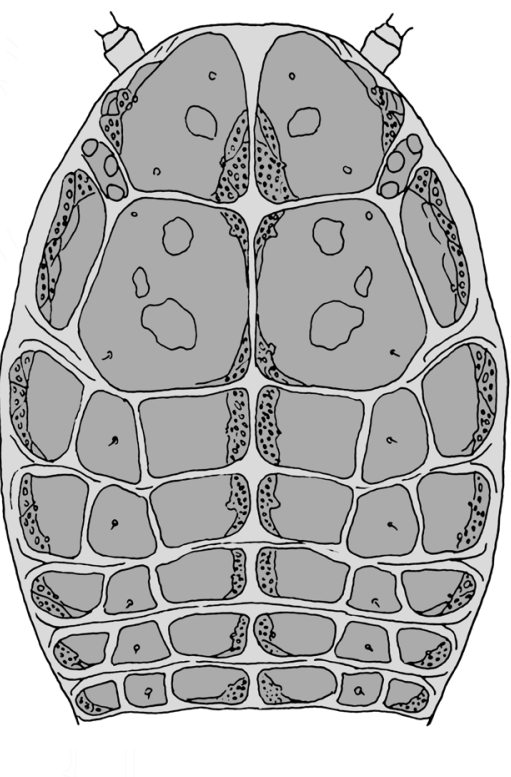

$0.1 \mathrm{~mm}$
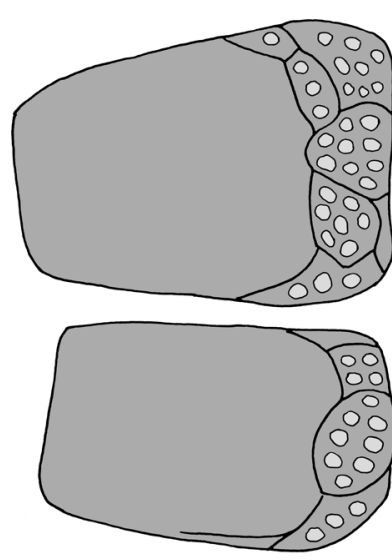

$0.5 \mathrm{~mm}$

B

C

Fig. 46. Adelges (Dreyfusia) nordmannianae (Eckstein, 1890). A. Gallicola (after Carter 1971, redrawn from Heie 2004). B-C. Hibernating first instar nymph (neosistens). B. Habitus, showing arrangement of sclerites plates and wax gland pits (after Schneider-Orelli 1947, modified and redrawn from Börner \& Heinze 1957). C. Spinal sclerites of meso- and metathorax, left side (after Pschorn-Walcher \& Zwölfer 1958, modified and redrawn).

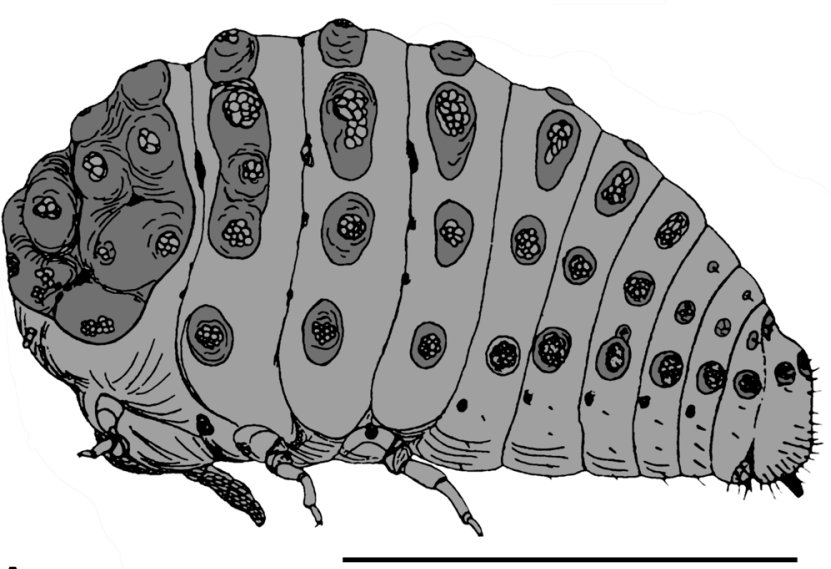

A

$0.5 \mathrm{~mm}$

Fig. 47. Adelges (Dreyfusia) piceae (Ratzeburg, 1843). A. Adult sistens (after Börner 1908, redrawn and modified). B-C. Hibernating first instar nymph (neosistens). B. Habitus, showing arrangement of sclerites and wax gland pits (after Schneider-Orelli 1947, modified and redrawn). C. Spinal sclerites of meso- and metathorax, left side (after Pschorn-Walcher \& Zwölfer 1958, modified and redrawn). 


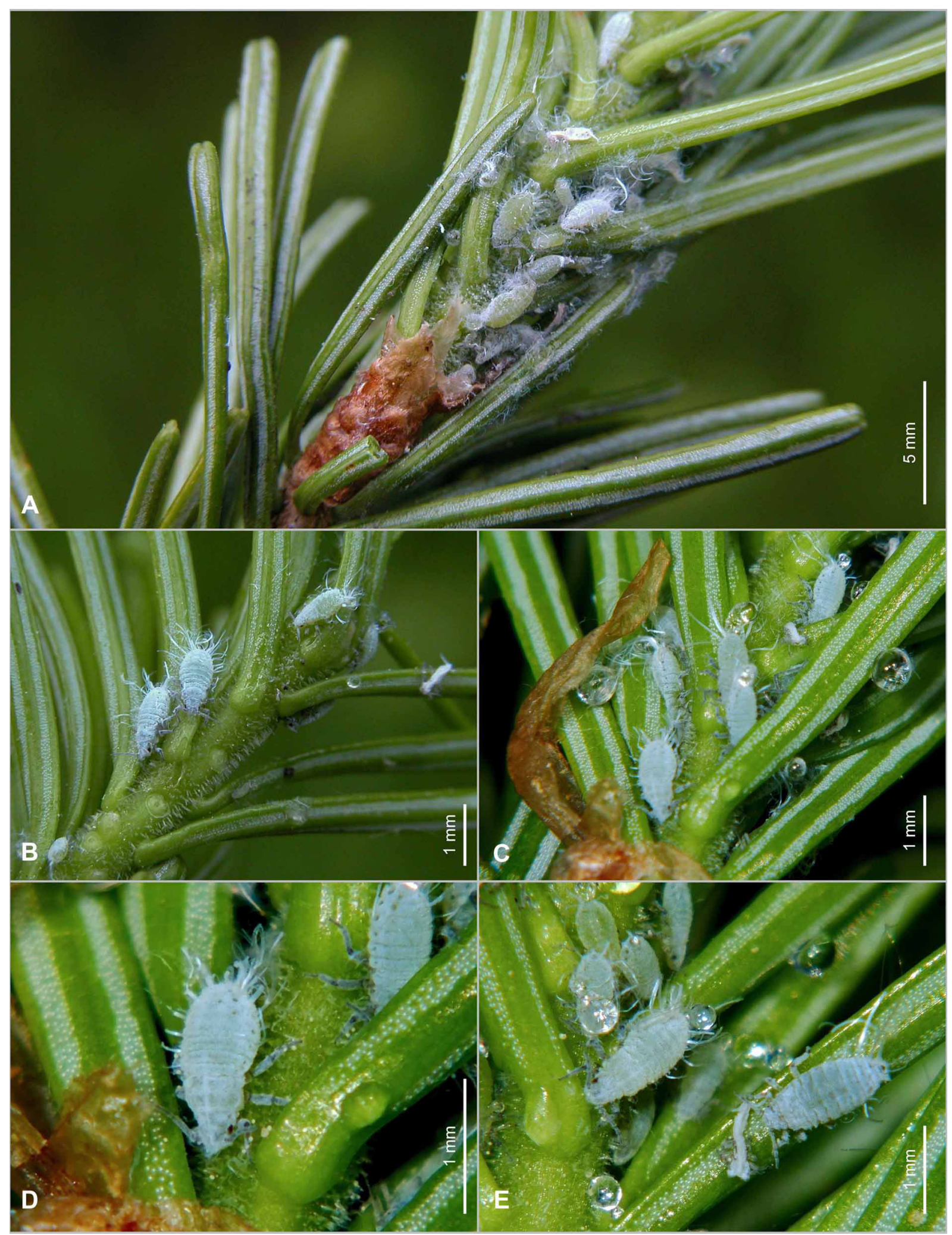

Fig. 48. Mindarus abietinus (Koch, 1857) on Abies sibirica. Colony on young shoot, mostly juvenile alatae. 

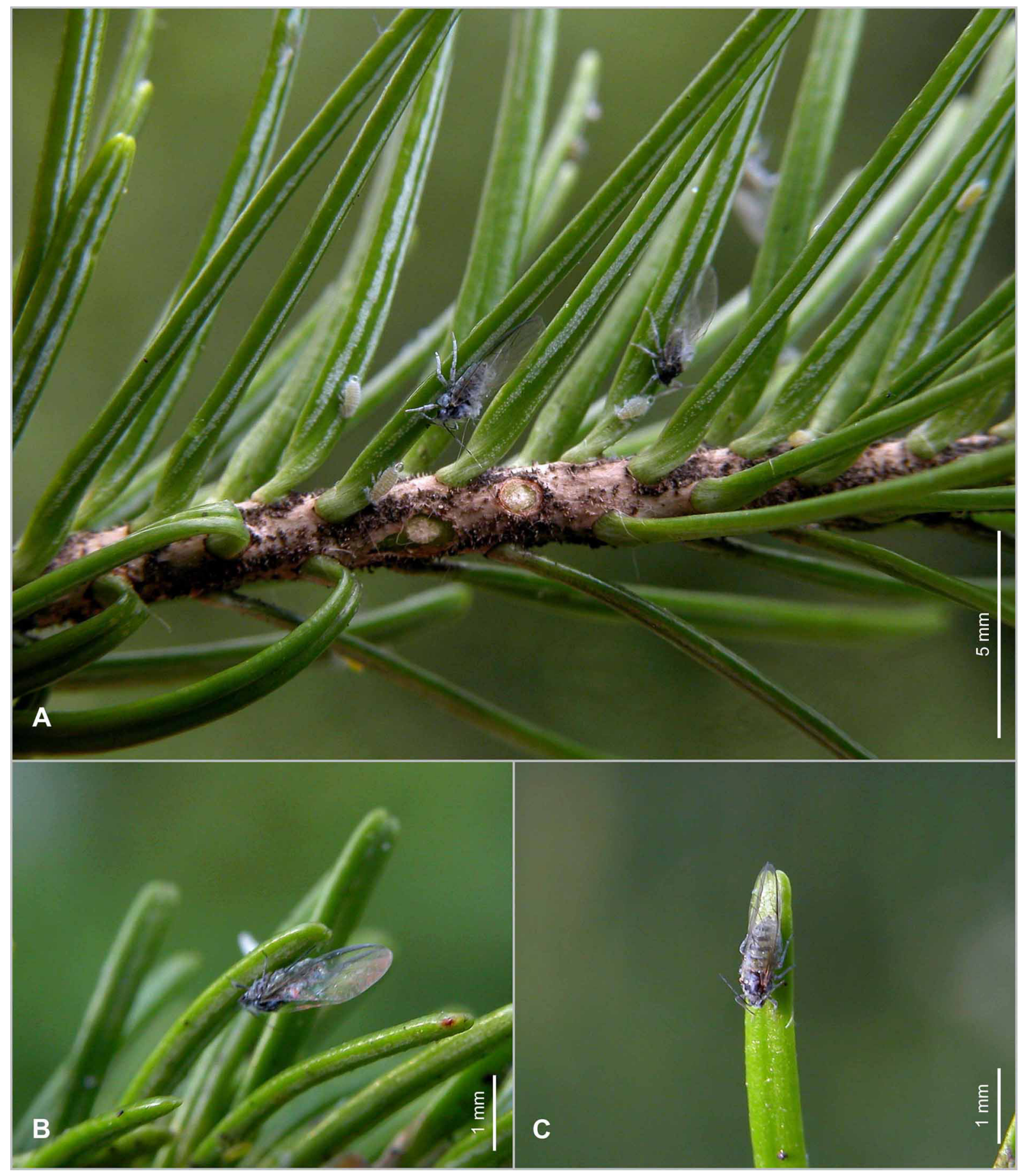

Fig. 49. Mindarus abietinus (Koch, 1857). Adult alatae and young oviparae on needles of Abies sibirica. 


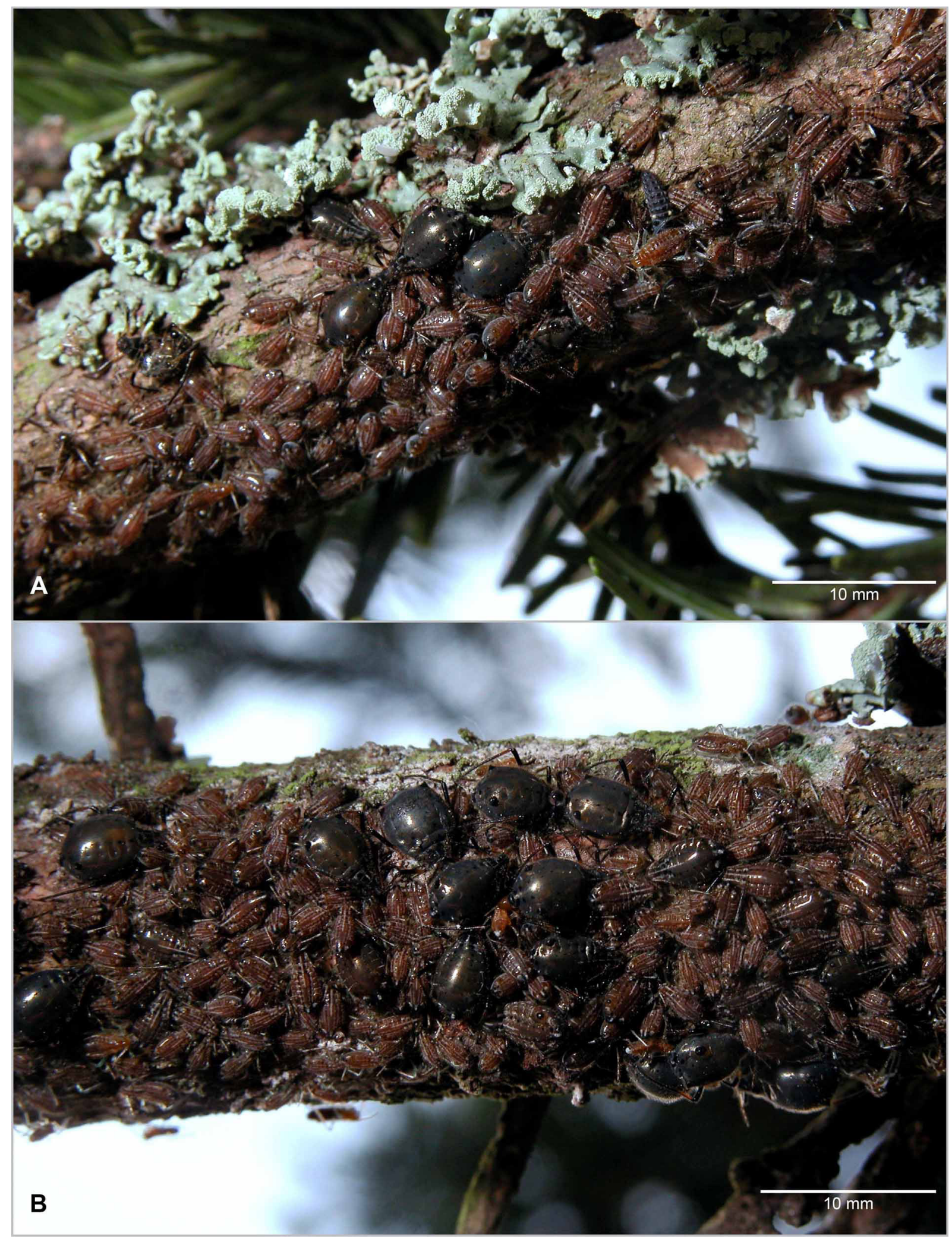

Fig. 50. Cinara (Cinara) confinis Koch, 1856. Colony on underside of branch of Abies sibirica. 


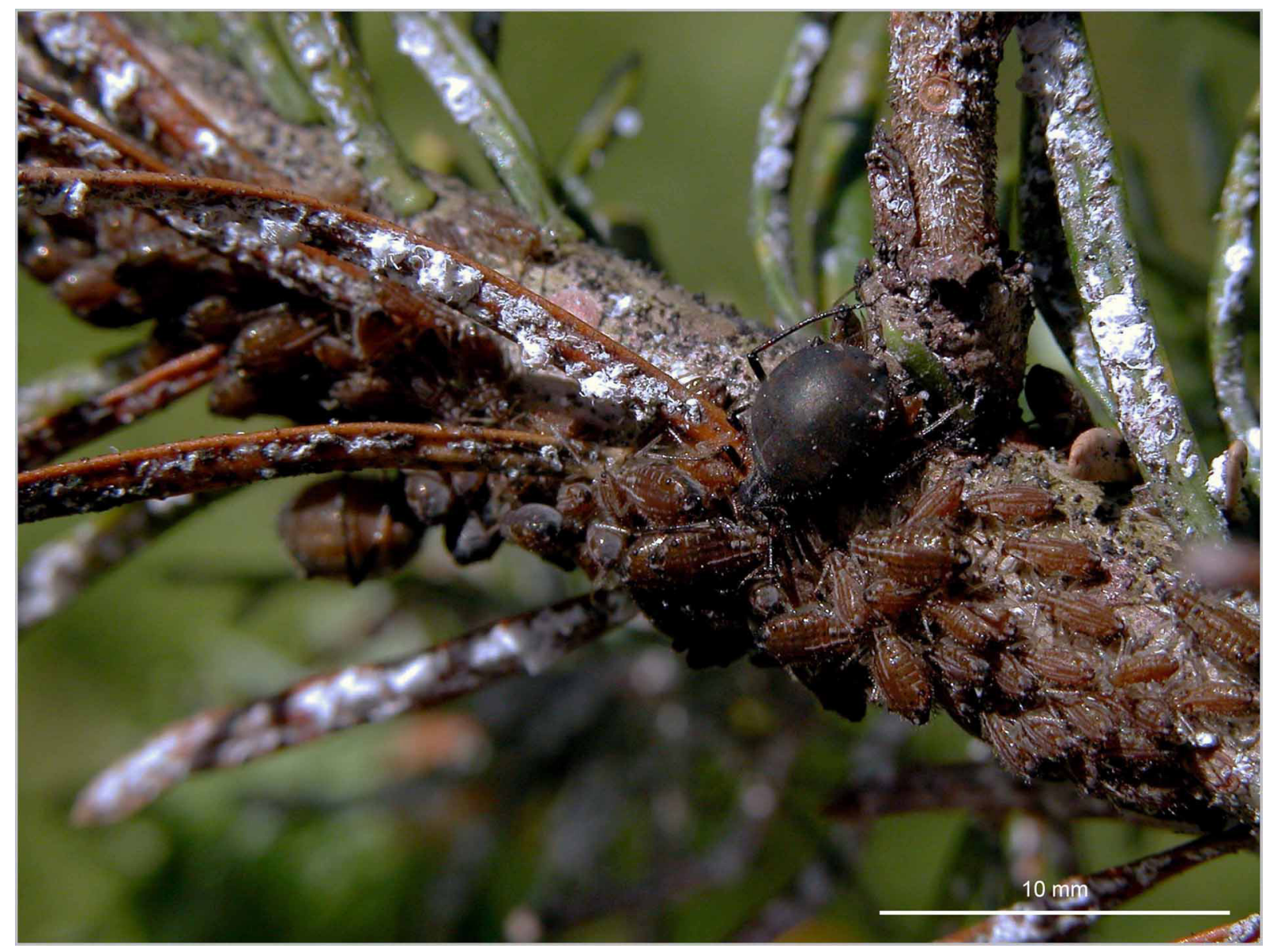

Fig. 51. Cinara (Cinara) confinis Koch, 1856. Colony on branch of Abies sibirica. Needles strongly infested by Adelges (Aphrastasia) pectinatae (Cholodkovsky, 1888). 


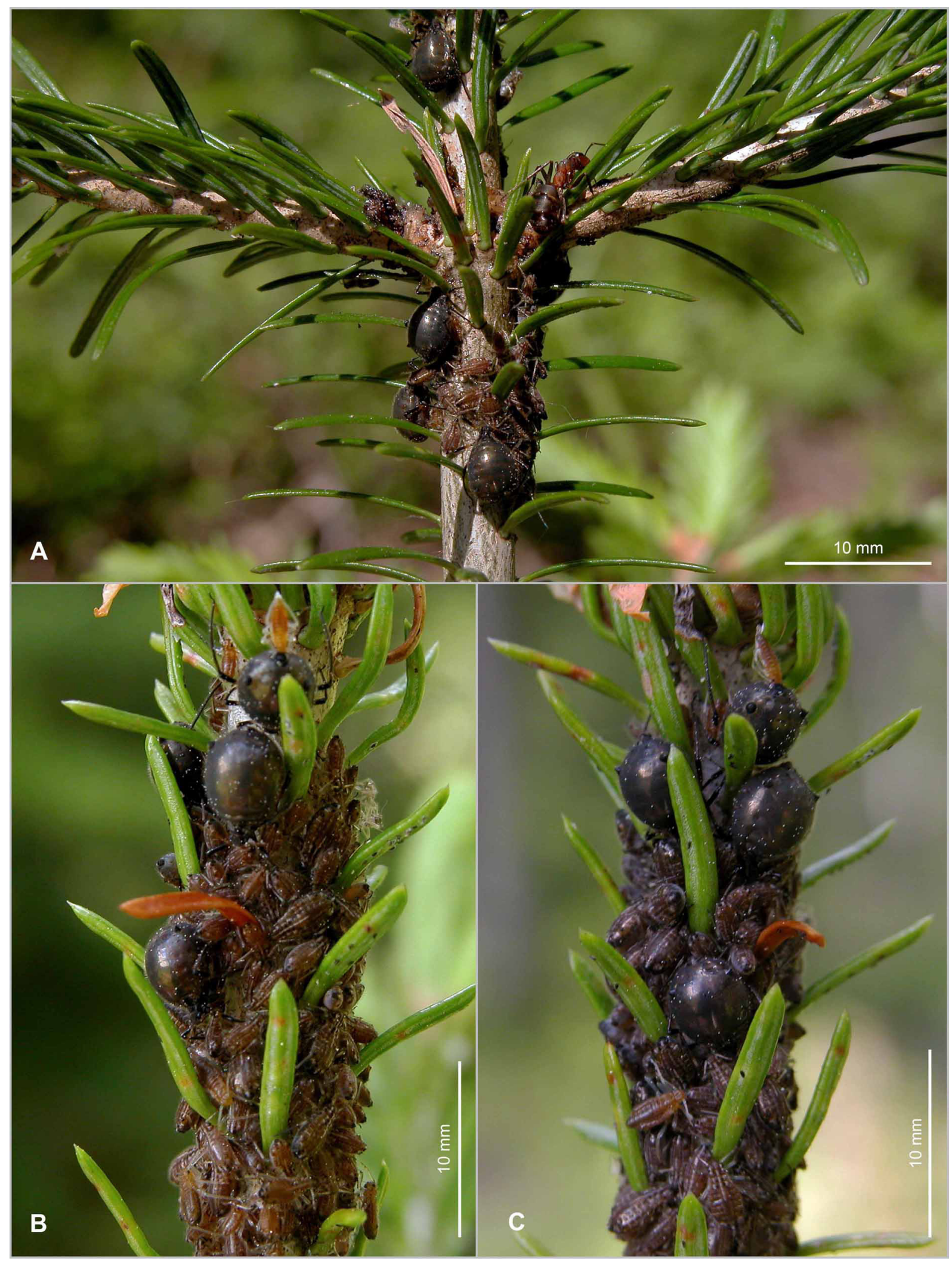

Fig. 52. Cinara (Cinara) confinis Koch, 1856 on young Abies sibirica. Colonies on 1-2 year-old parts of main shoot, attended by Formica rufa Linnaeus, 1761. B-C. Needles with brown spots and overwintered nymphs from the previous year's infestation of Adelges (Aphrastasia) pectinatae (Cholodkovsky, 1888) 


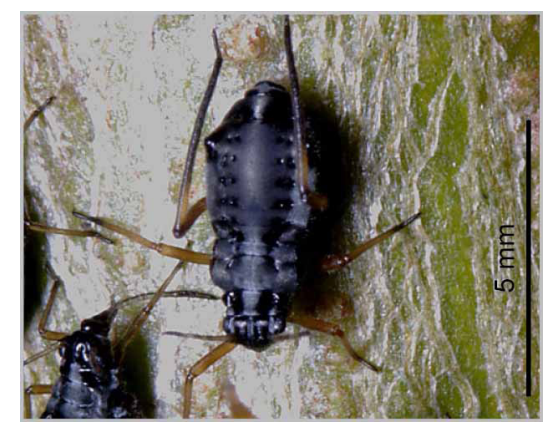

Fig. 53. Cinara (Cinara) curvipes (Patch, 1912), juvenile alata on Abies sp. (from Dransfield \& Brightwell 2015, licensed under Creative Commons Attribution 3.0, downloaded 15 Sept. 2015). 


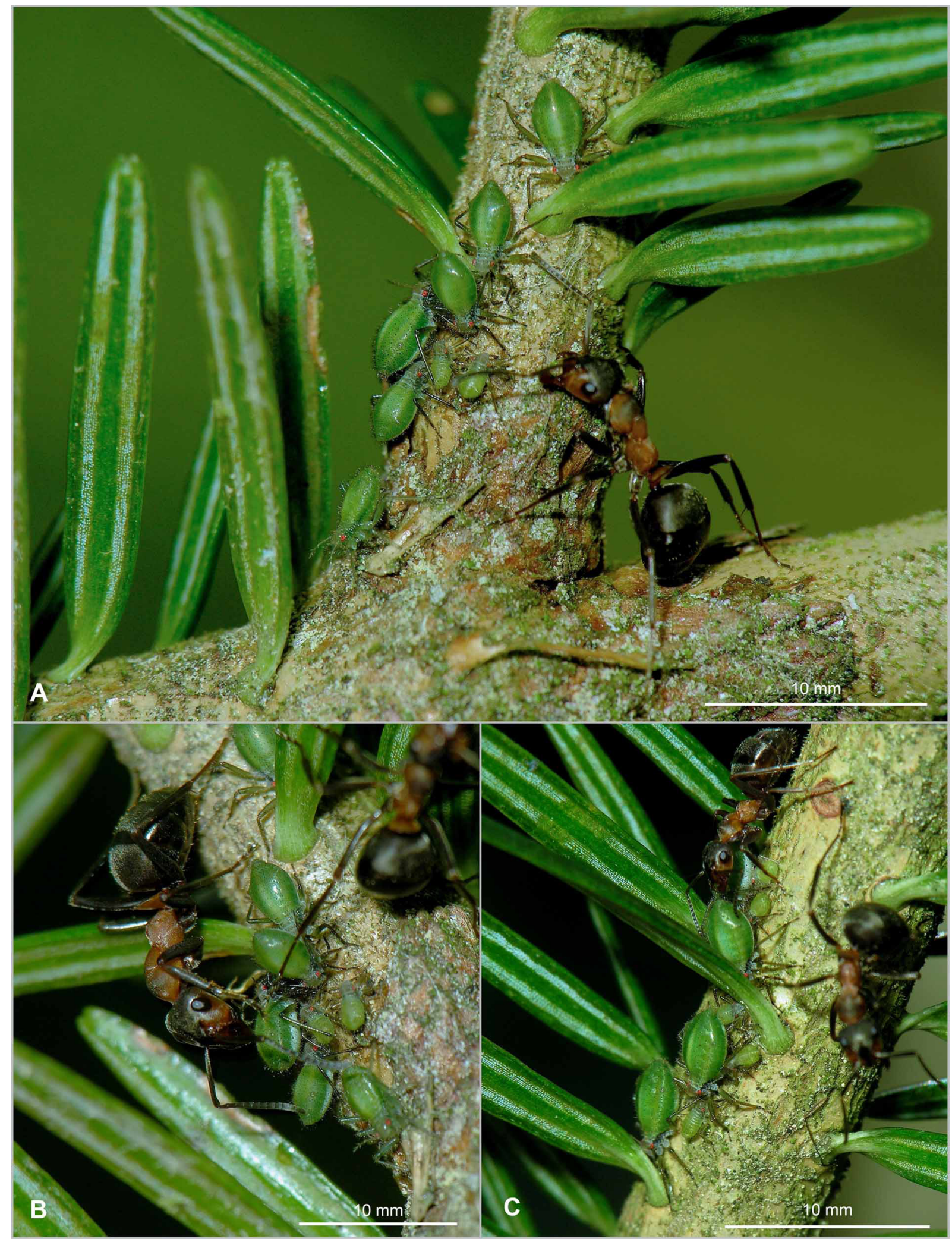

Fig. 54. Cinara (Cinara) pectinatae Nördlinger, 1880. Colonies (ad. and juv.) on trunk and branches of young Abies alba, attended by Formica polyctena Förster, 1850. 


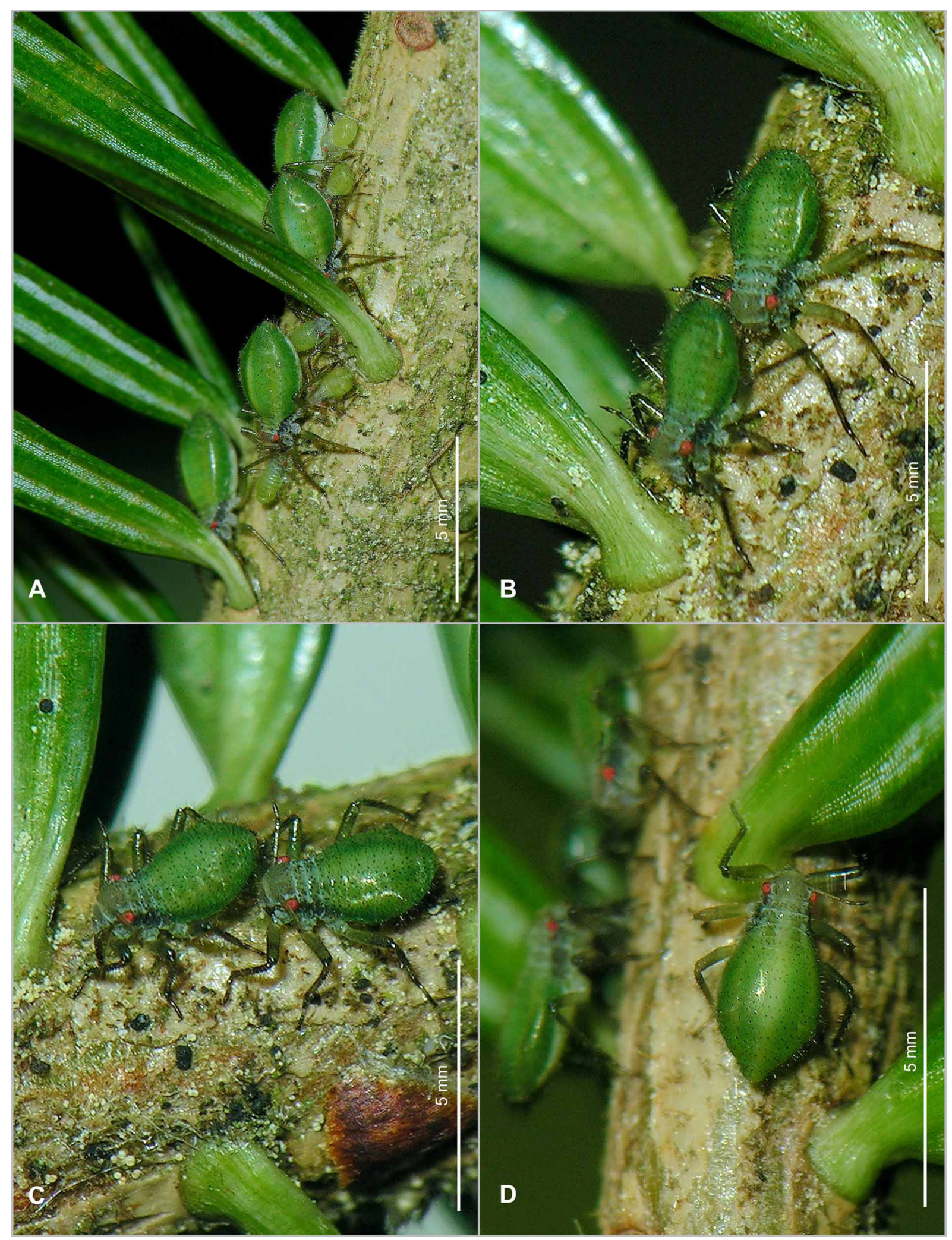

Fig. 55. Cinara (Cinara) pectinatae Nördlinger, 1880. Colonies (mostly juv.) on trunk and branches of young Abies alba. 

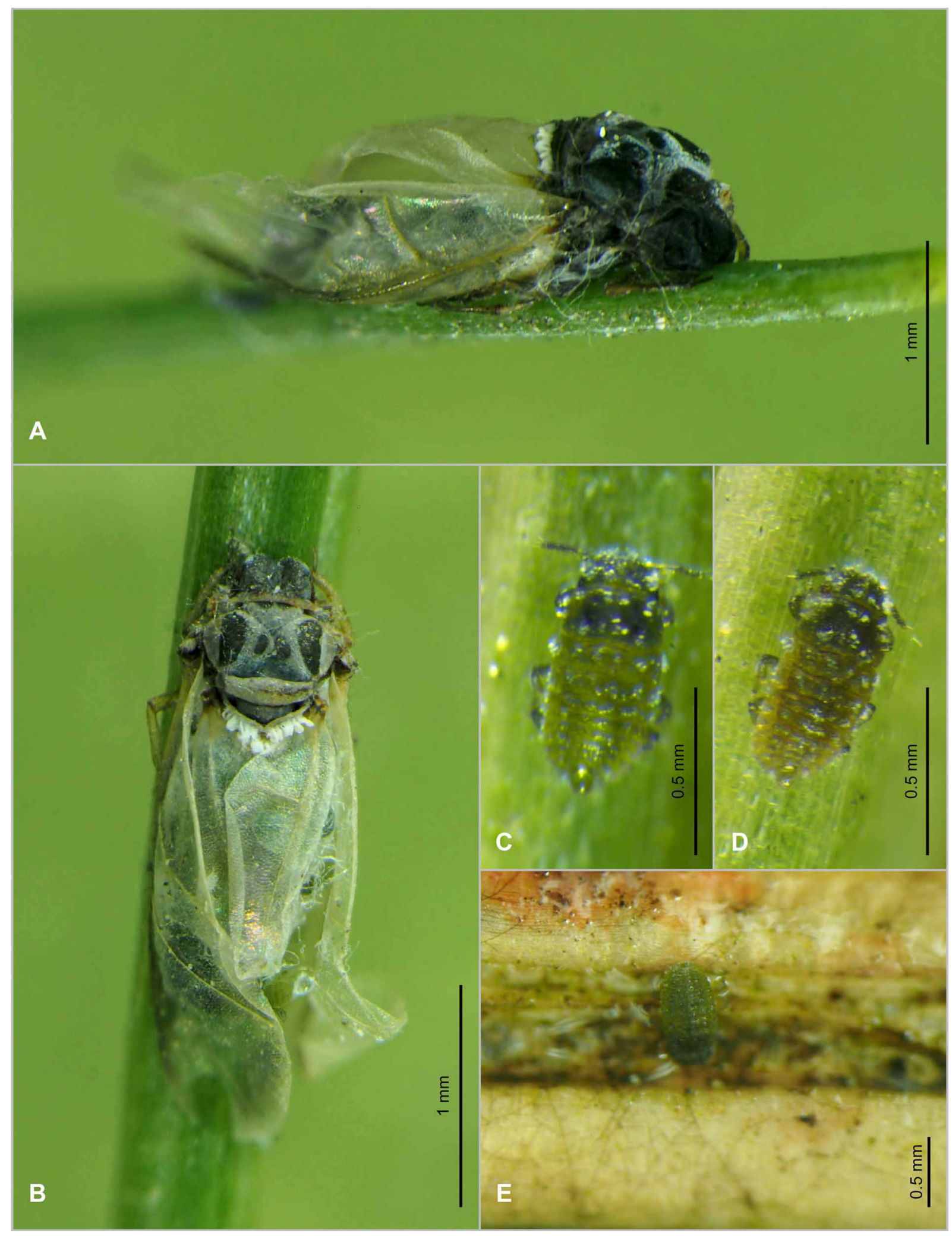

Fig. 56. Adelges (Sacciphantes) viridis (Ratzeburg, 1843) on Larix sibirica. A-B. Dead gallicolae on upper side of needle, with eggs and newly hatched neosistens under the wings. C-D. Neosistens on needle. E. Neosistens on bark of twig. 


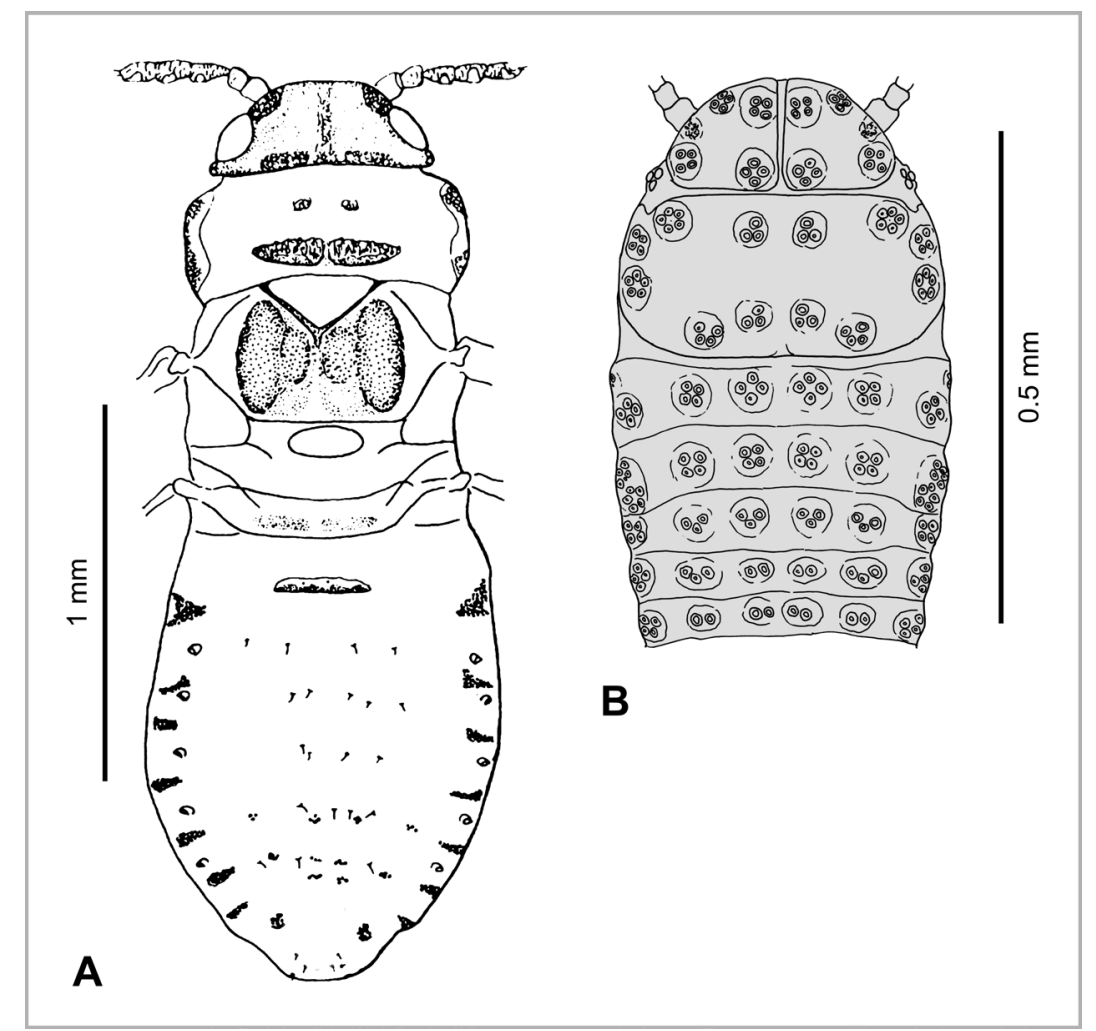

Fig. 57. Adelges (Sacciphantes) viridis (Ratzeburg, 1843). A. Sexupara. Note wax glands on prothorax (absent in gallicola) (after Carter 1971, redrawn from Heie 2004). B. Neosistens (after Schneider-Orelli 1947, modified). 


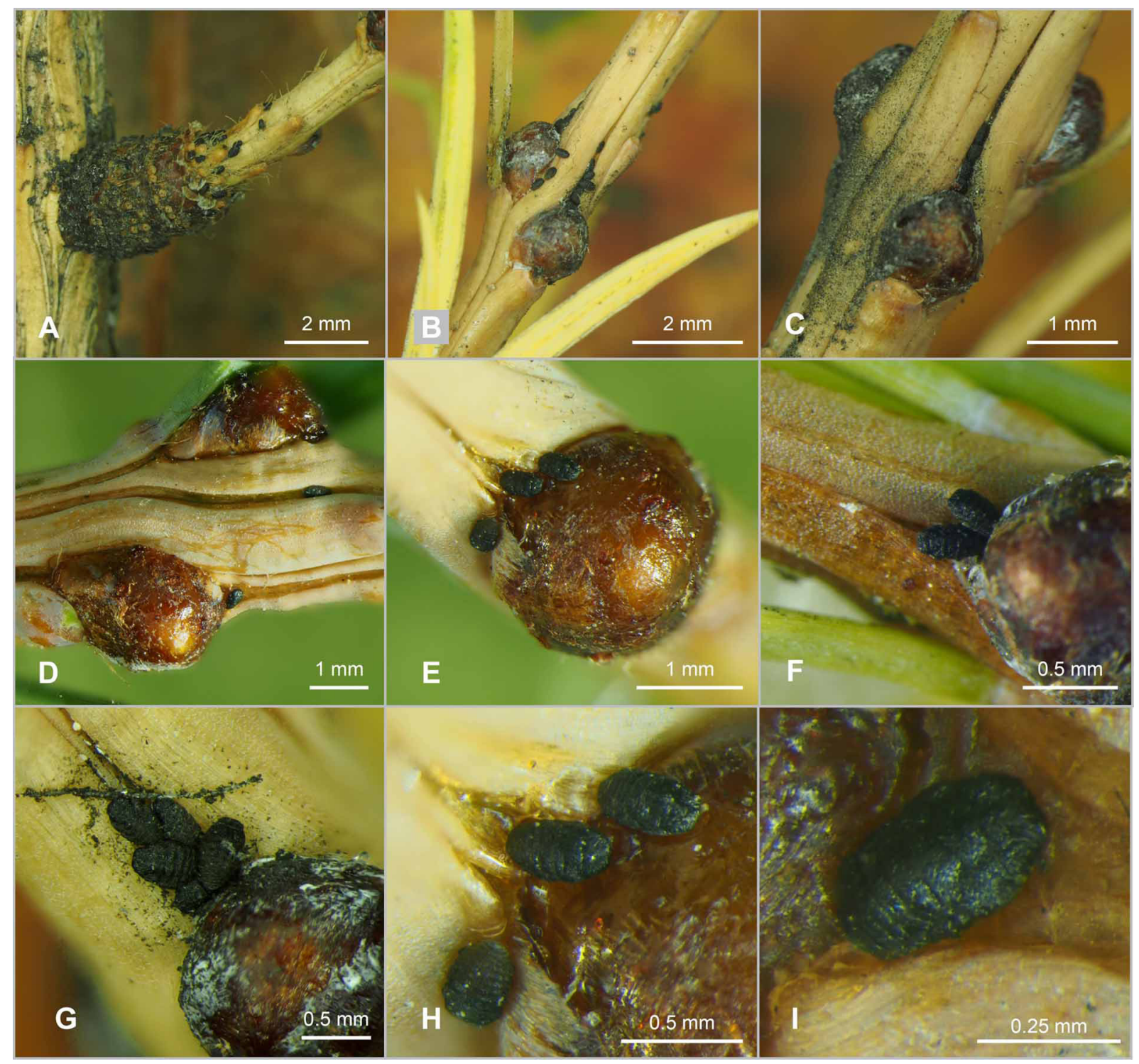

Fig. 58. Adelges (Adelges) laricis Vallot, 1836. Neosistentes in winter on twigs of Larix sibirica. 

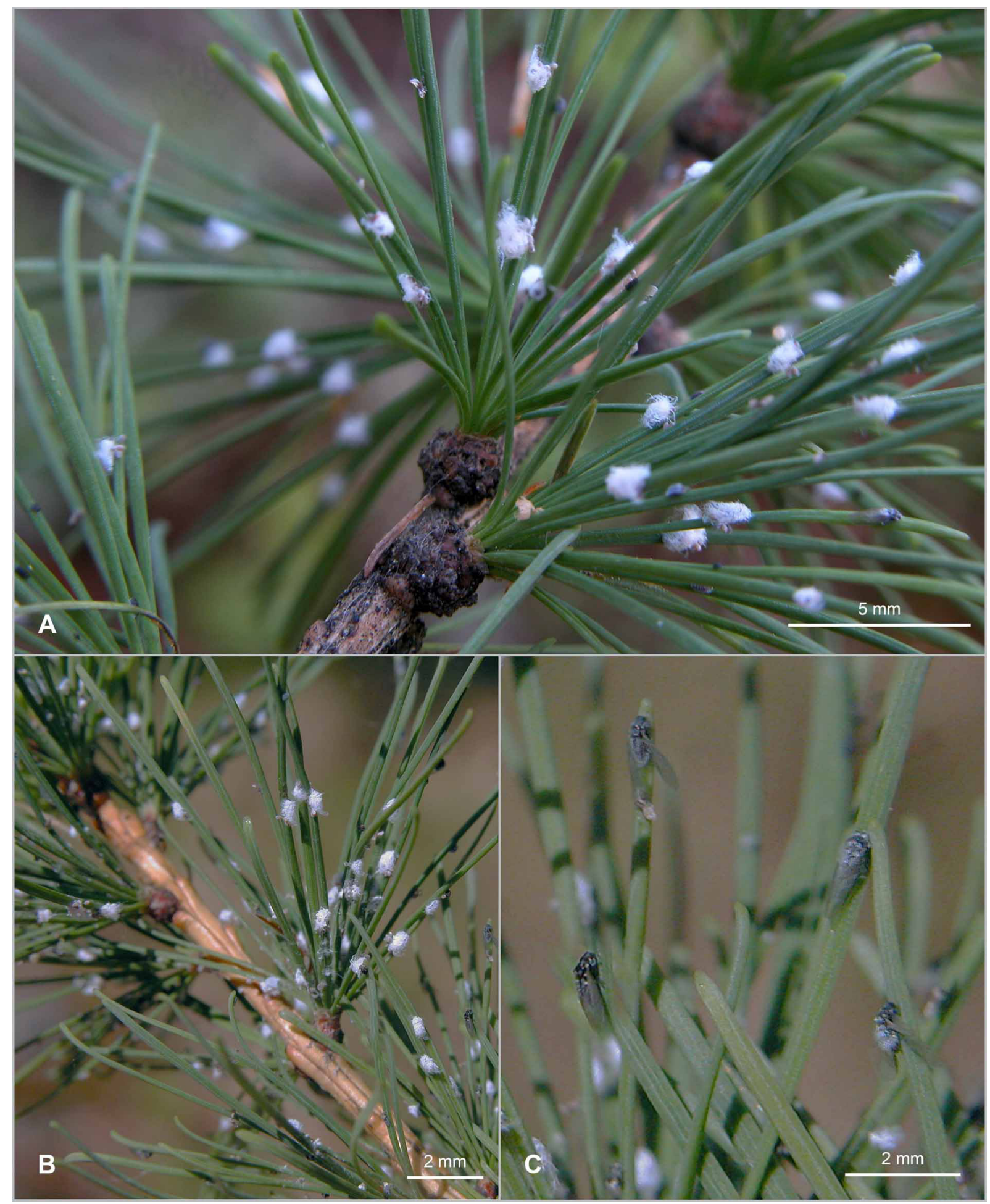

Fig. 59. Adelges (Adelges) laricis Vallot, 1836. Apterous and alate progredientes on Larix sibirica. 


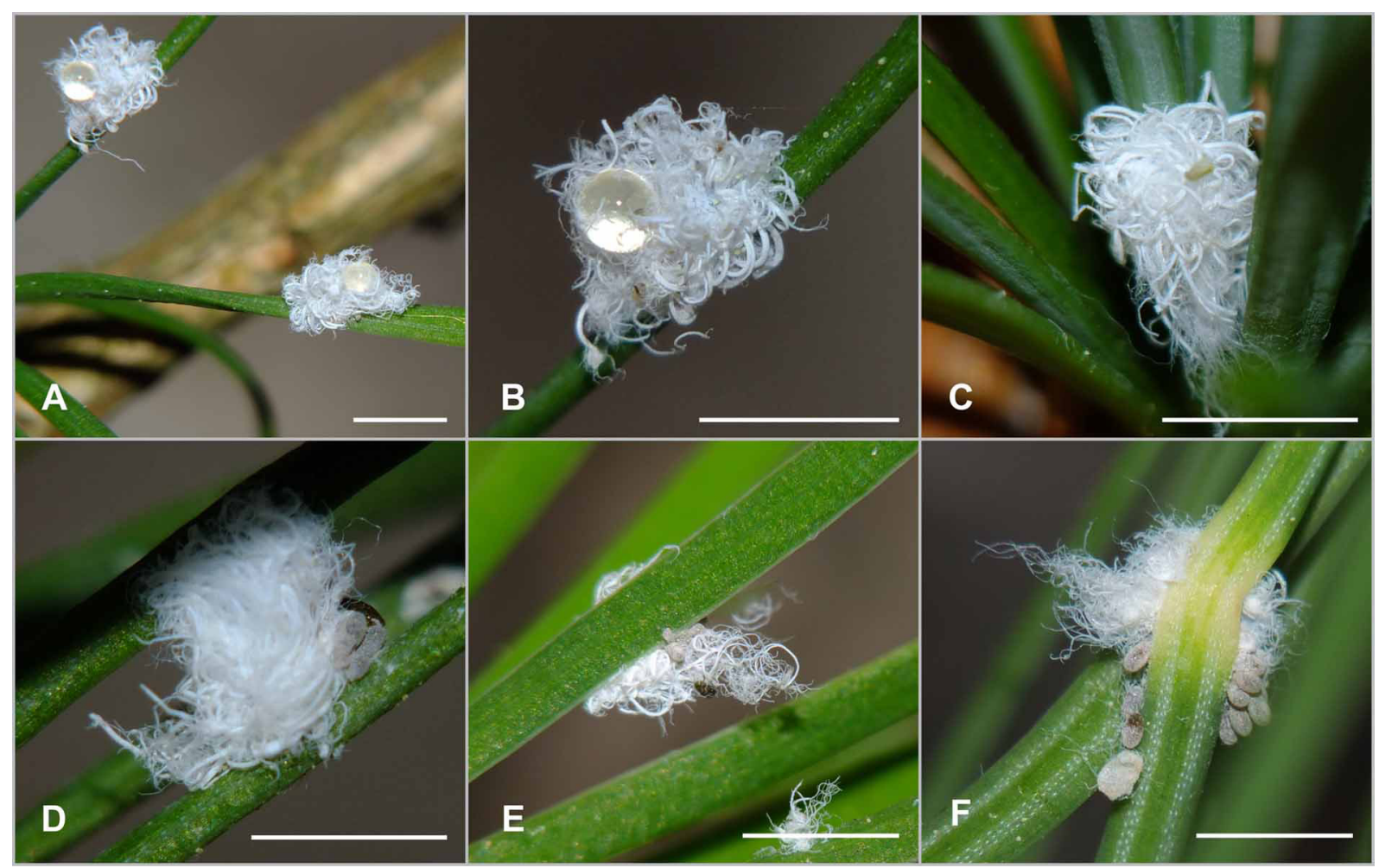

Fig. 60. Adelges (Adelges) laricis Vallot, 1836. Adult apterous progredientes with eggs and honeydew droplets on Larix sibirica. (A-D) and Larix kaempferi (E-F). Scale bars: about $2.5 \mathrm{~mm}$.

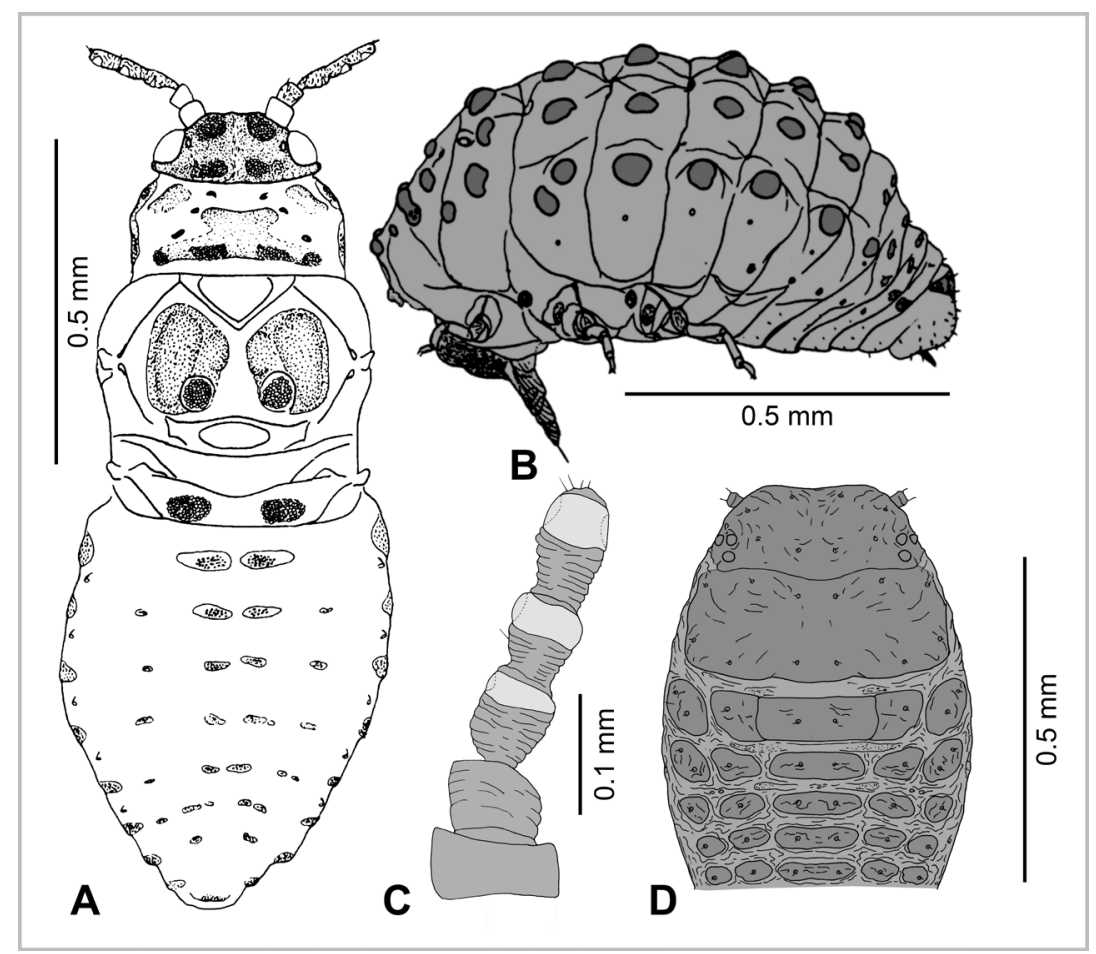

Fig. 61. Adelges (Adelges) laricis Vallot, 1836. A. Sexupara (after Carter 1971, redrawn from Heie 2004). B. Adult apterous progrediens (after Börner 1908, redrawn from Heie 2004). C. Antenna of gallicola (after Annand 1928, modified). D. Neosistens (after Schneider-Orelli 1947, modified). 


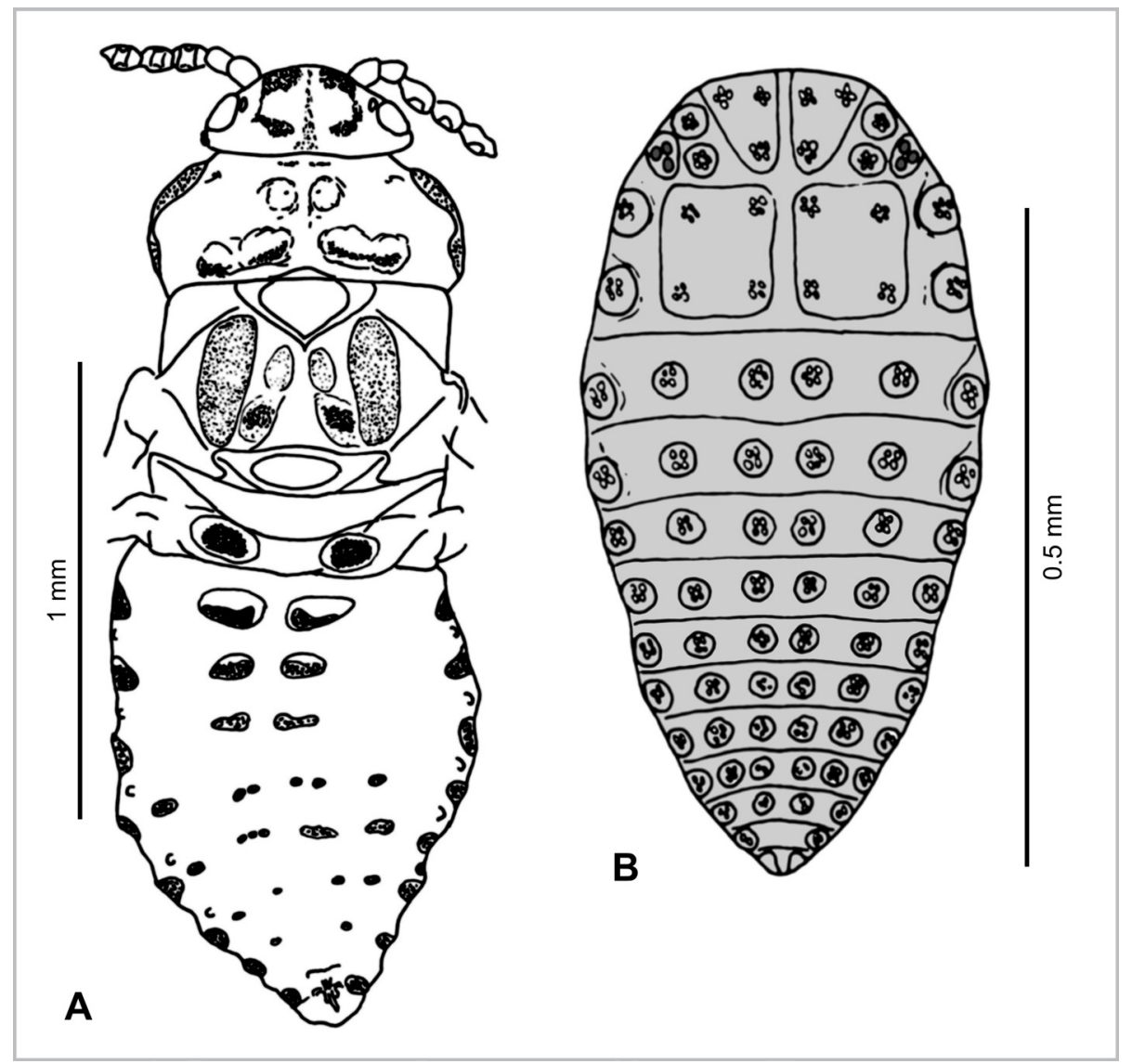

Fig. 62. Adelges (Cholodkovskya) viridanus (Cholodkovsky, 1896). A. Alata (after Carter 1971, redrawn from Heie 2004). B. Neosistens (after Inouye 1953). 

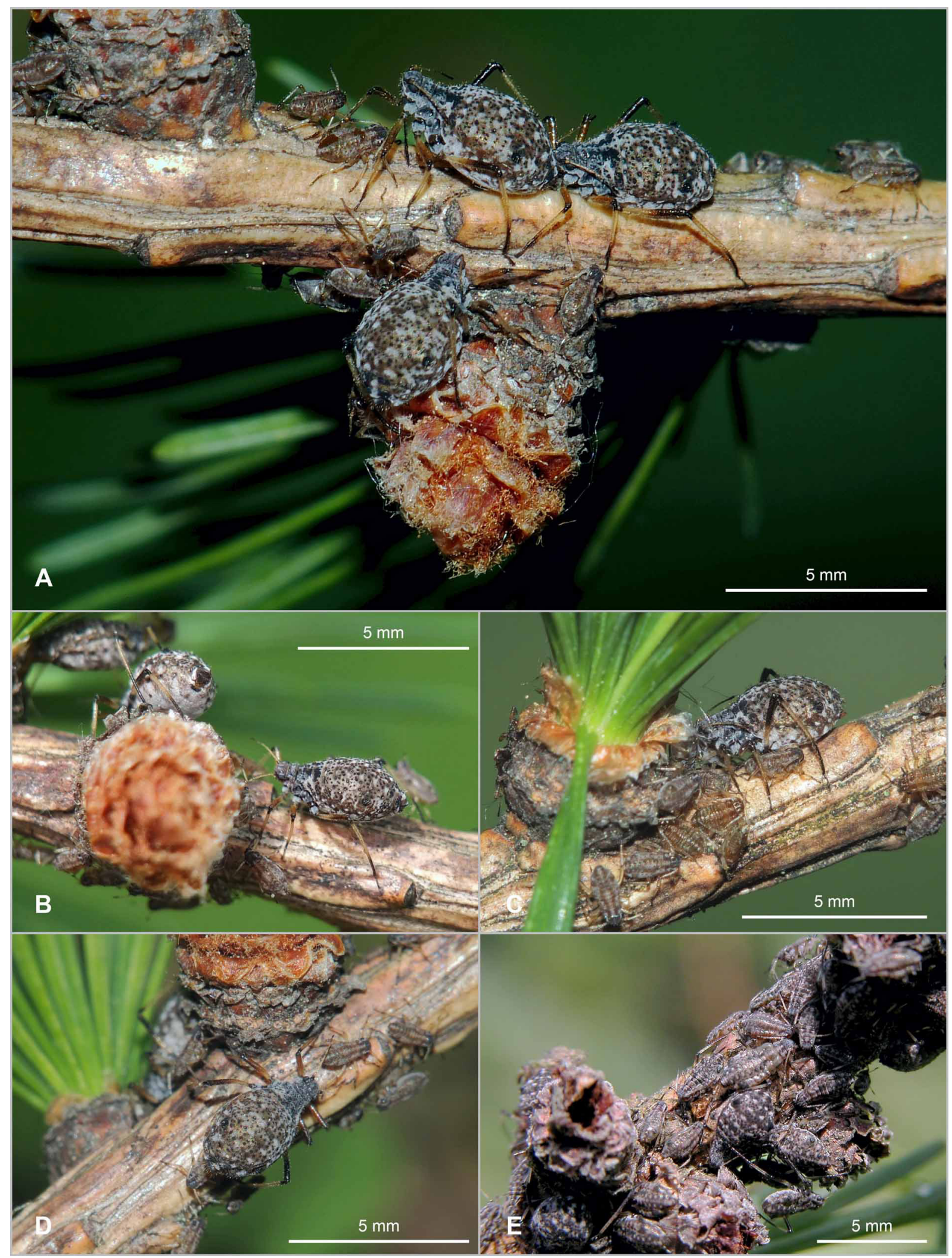

Fig. 63. Cinara (Cinara) laricis (Hartig, 1839). Adult apterae with juvenile apterae and alatae. A-D. On Larix decidua. E. On L. sibirica. 


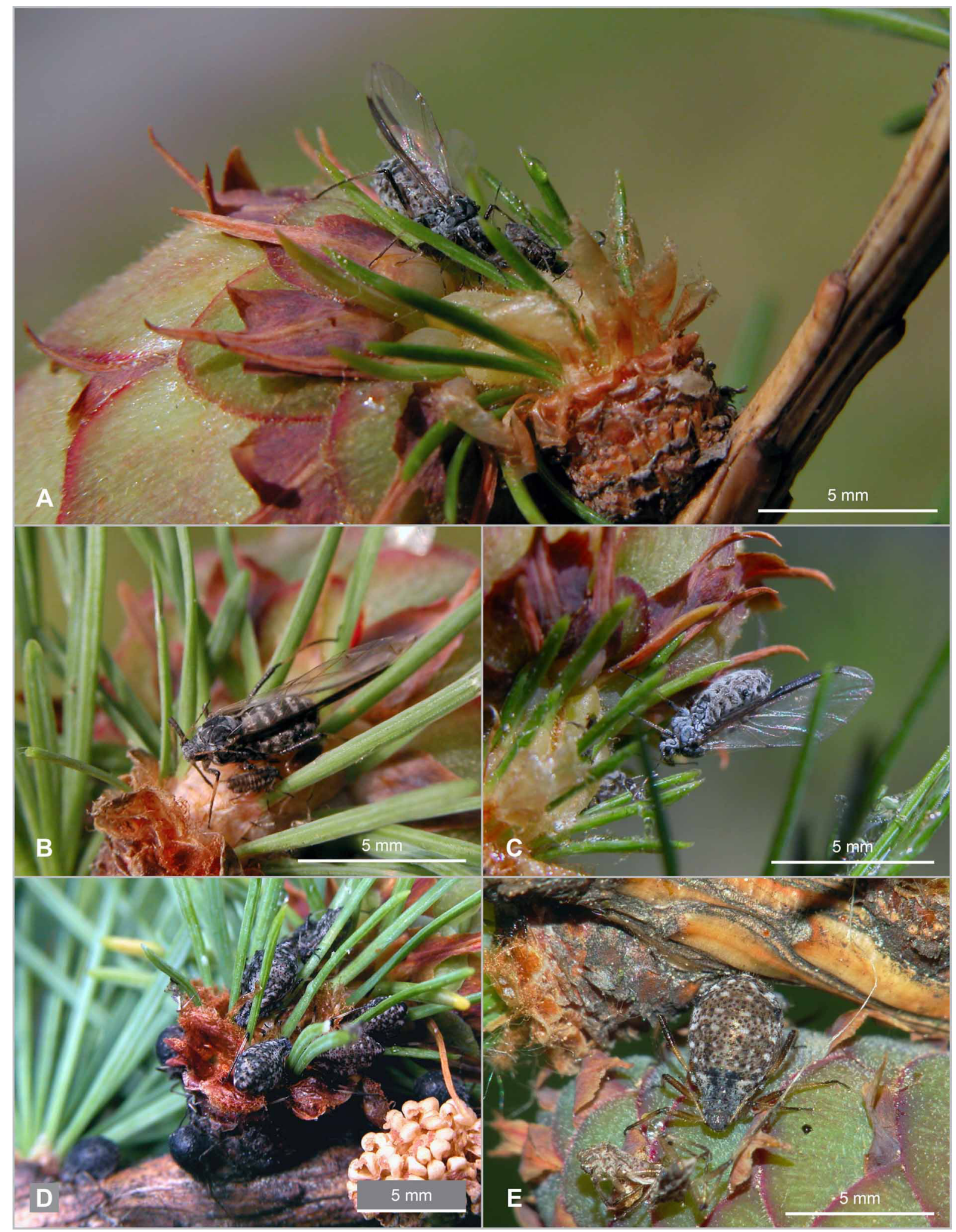

Fig. 64. Cinara (Cinara) laricis (Hartig, 1839). Alatae and apterae with nymphs on young cones of Larix decidua. Note the wax-powdered venter (in C). 


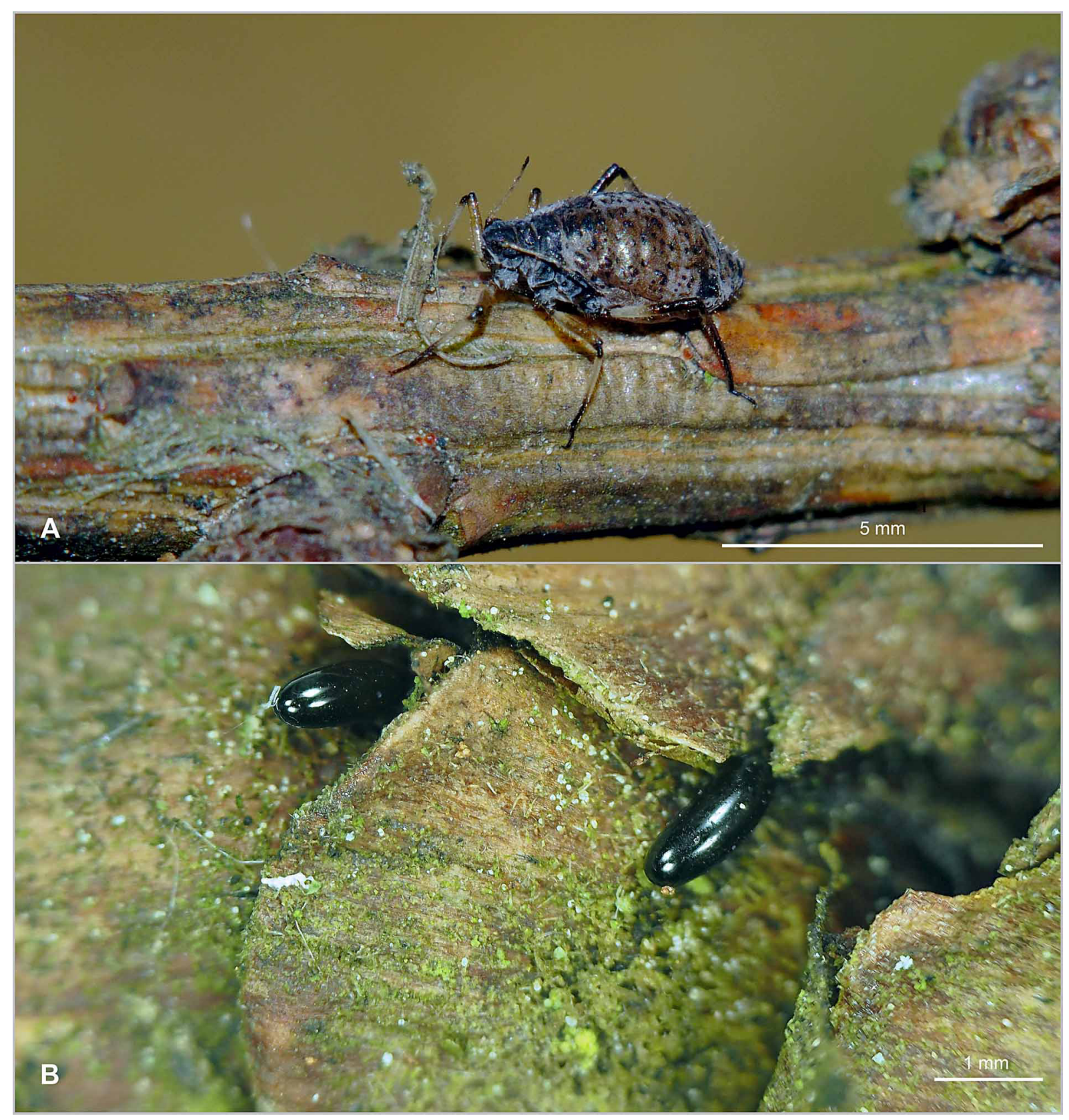

Fig. 65. Cinara (Cinara) laricis (Hartig, 1839) on Larix decidua in late autumn. A. Ovipara on twig. B. Eggs between scales of previous year's cone. Preanal wax ring absent in the ovipara, hence the eggs without wax cover. 


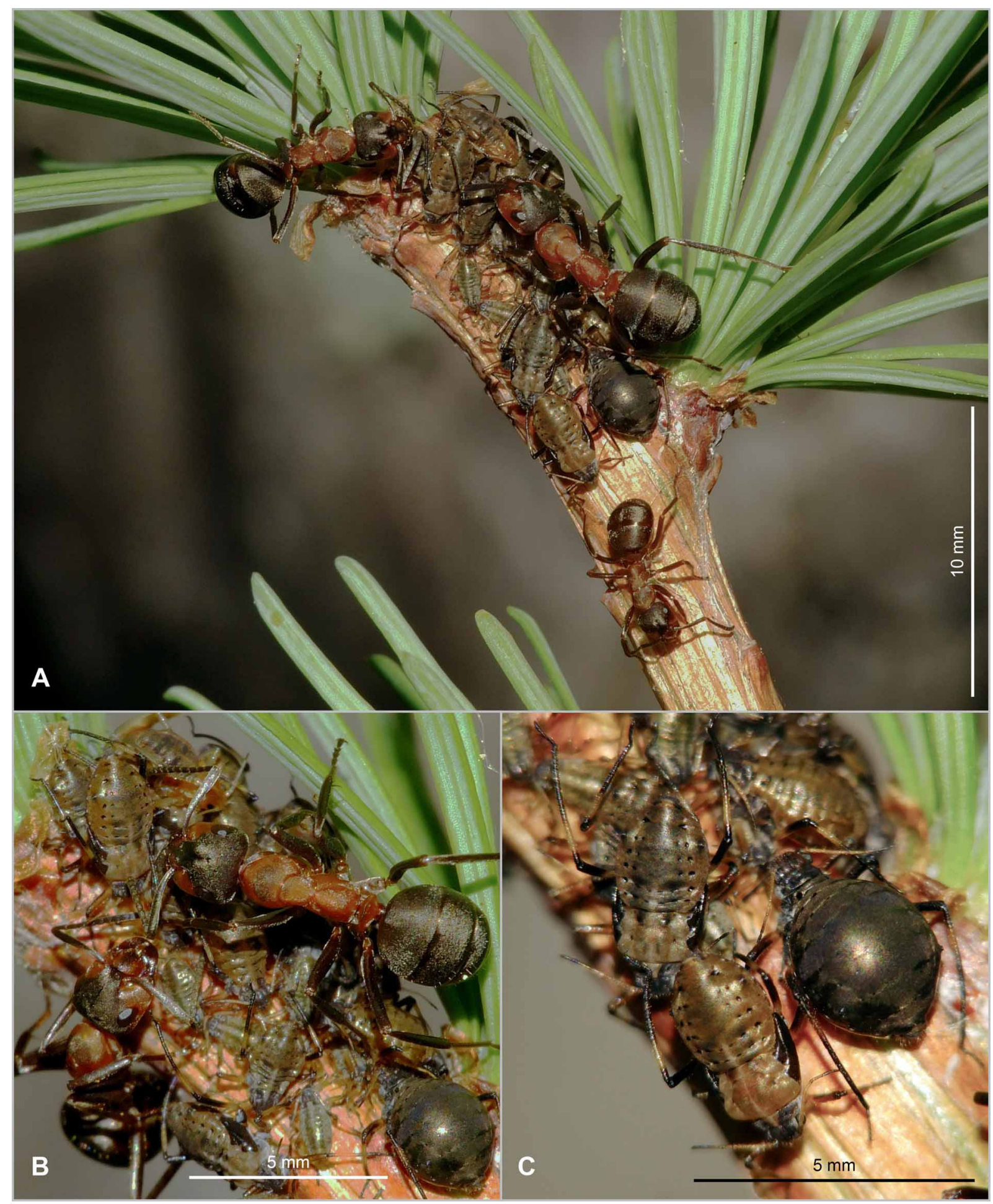

Fig. 66. Cinara (Cinara) cuneomaculata (del Guercio, 1909). Fundatrix with young (mostly alatae) on twig of Larix kaempferi, attended by Formica rufa Linnaeus, 1761. 


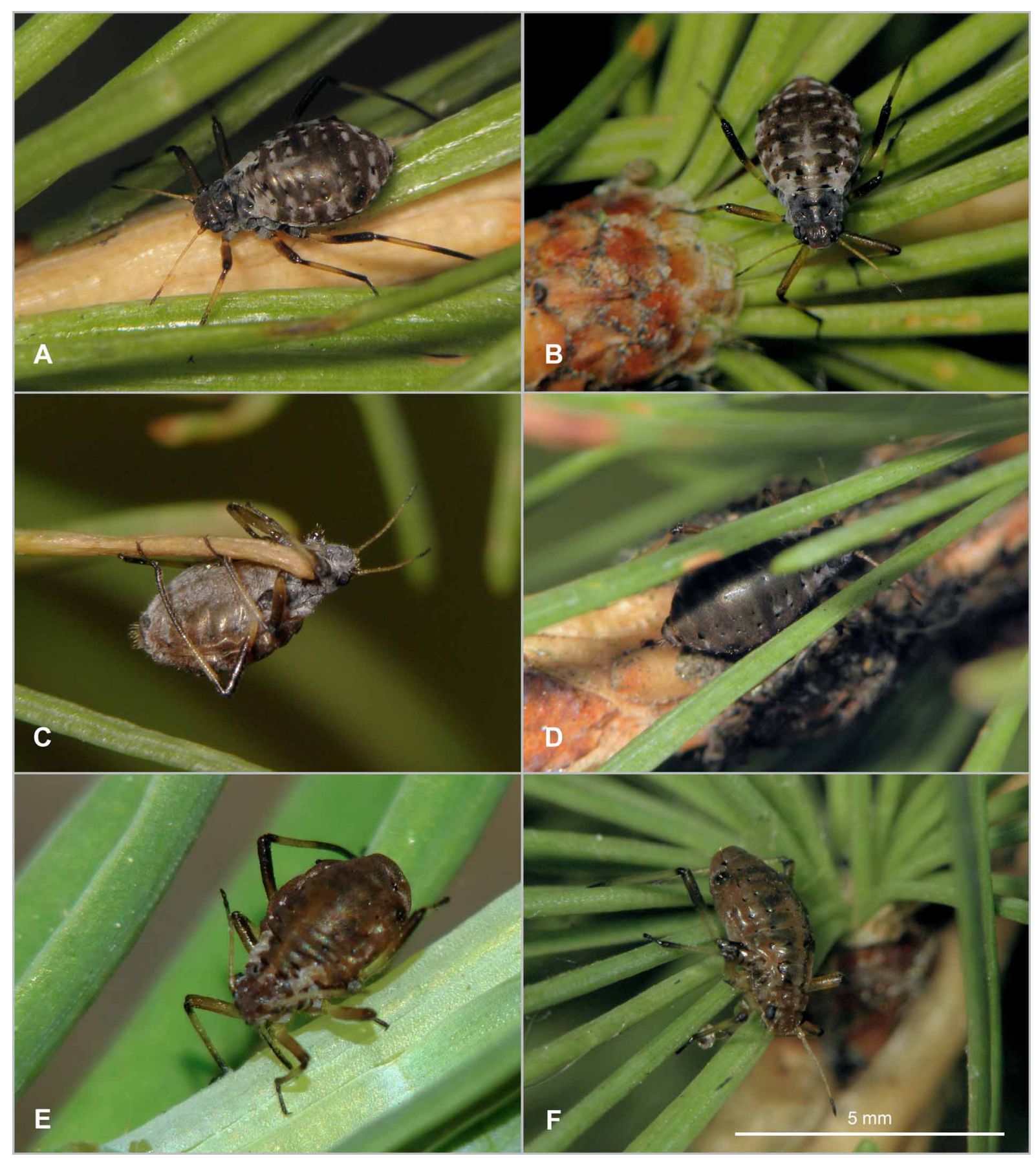

Fig. 67. Cinara (Cinara) cuneomaculata (del Guercio, 1909). A-D, F. Among needles of Larix sibirica. E. Among needles of L. kaempferi. Note the variation in the wax pattern. 


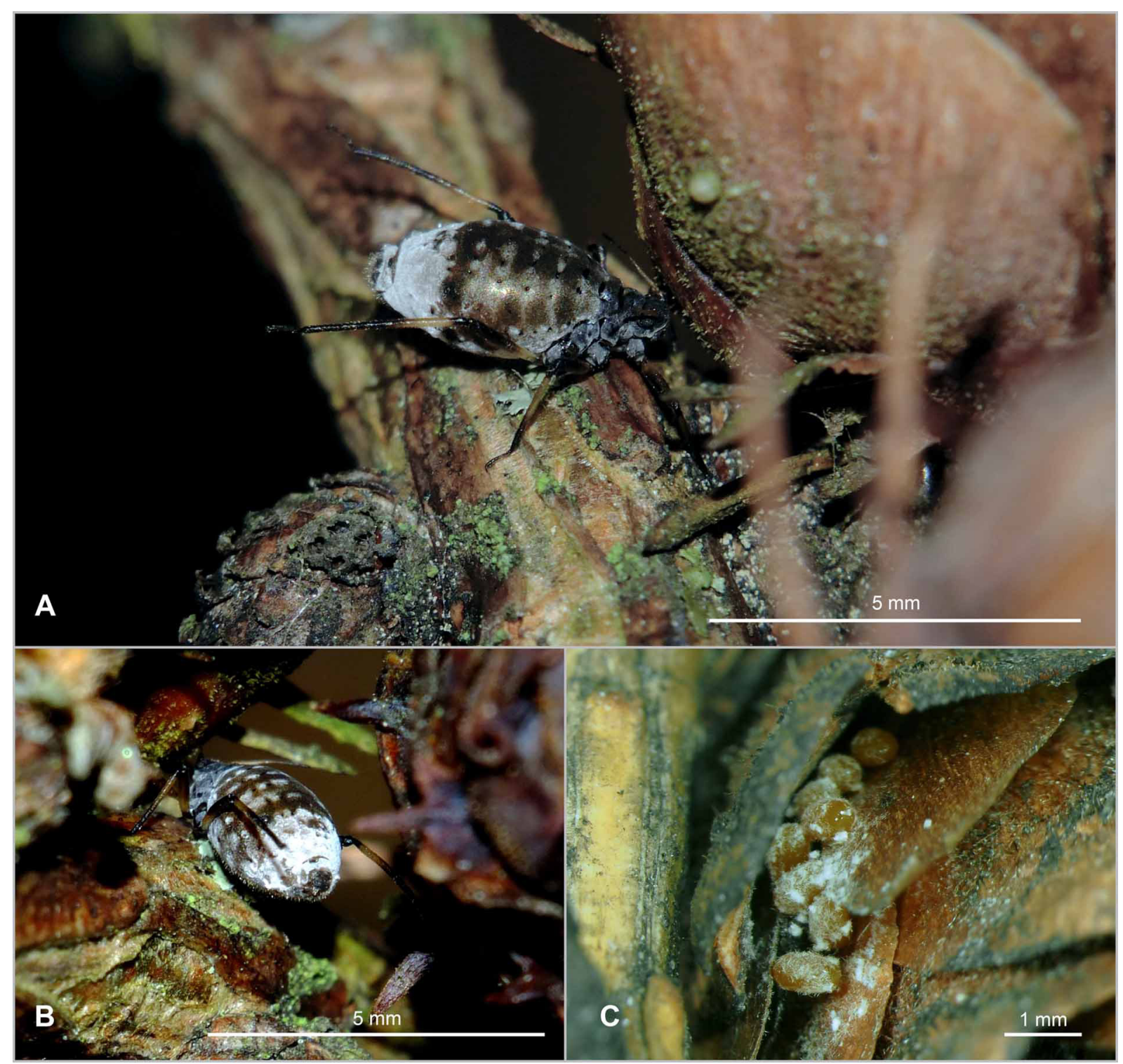

Fig. 68. Cinara (Cinara) cuneomaculata (del Guercio, 1909) on Larix decidua in late autumn. A-B. Oviparae on old twigs and cones. C. Eggs between scales of old cone. Note the preanal wax ring of the oviparae and the wax powder on the eggs.

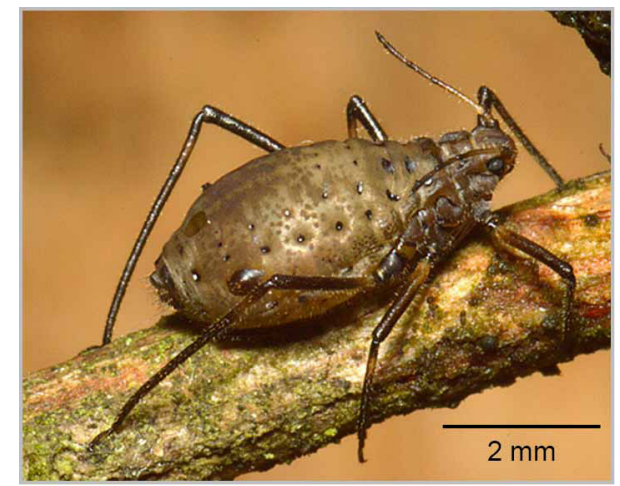

Fig. 69. Cinara (Cinara) kochiana (Börner, 1939). Aptera (from Dransfield \& Brightwell 2015, licensed under CC BY 3.0 US, https://creativecommons.org/licenses/by/3.0/us/ legalcode, downloaded 27 Oct. 2015). 


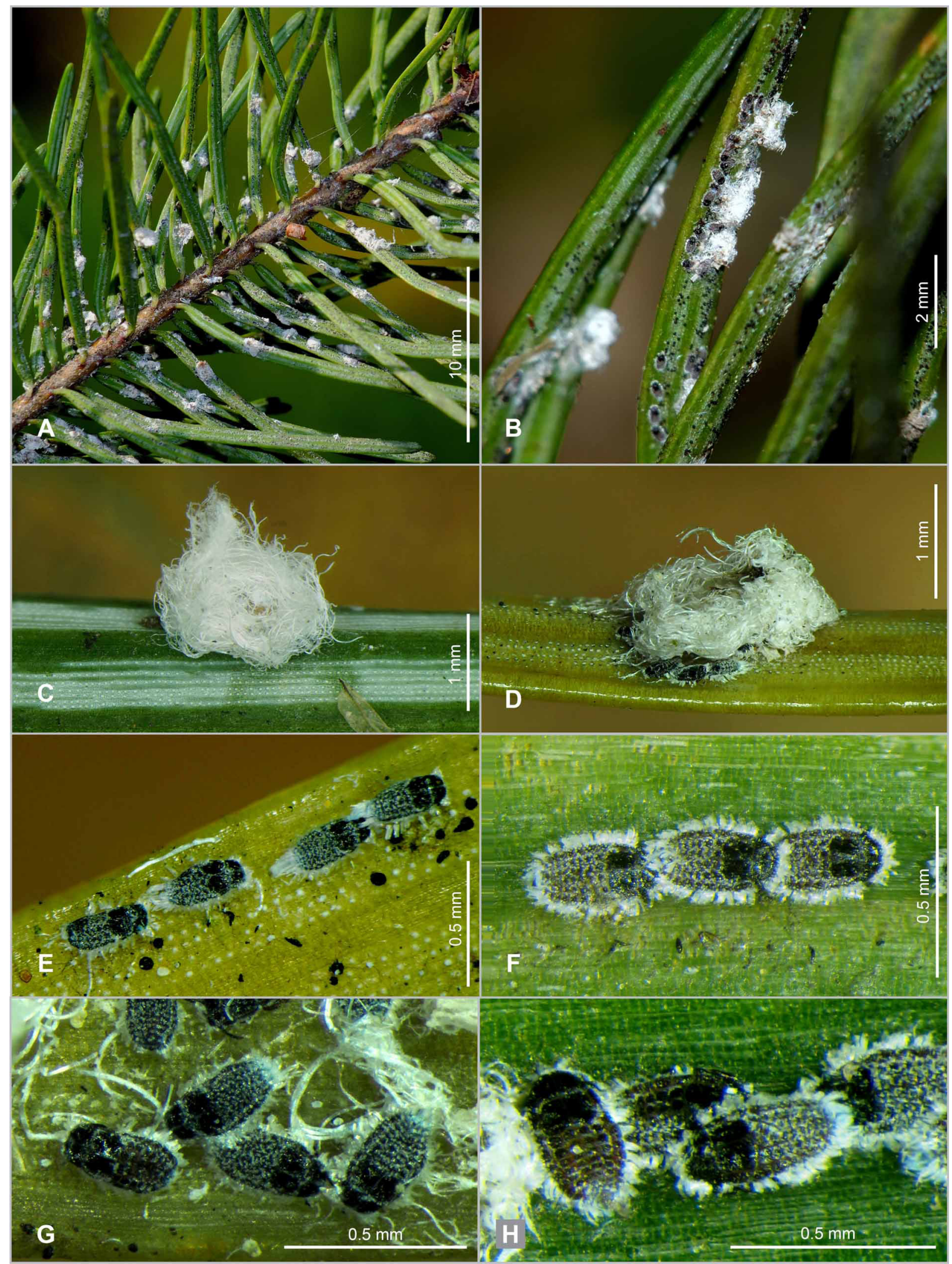

Fig. 70. Adelges (Gilletteella) cooleyi (Gillette, 1907). Apterae and neosistentes on needles of Pseudotsuga menziesii. 


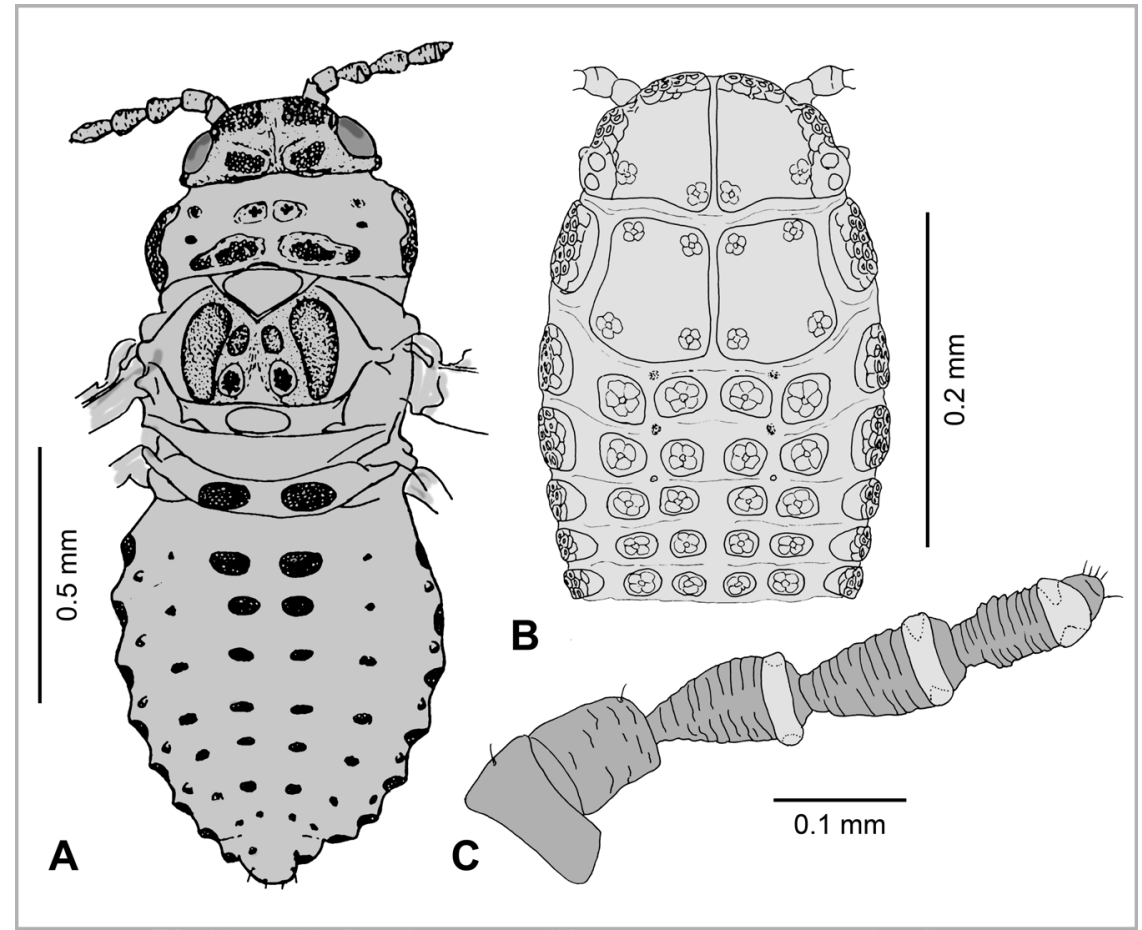

Fig. 71. Adelges (Gilletteella) cooleyi (Gillette, 1907). A. Gallicola (after Carter 1971, redrawn from Heie 2004). B. Neosistens (after Schneider-Orelli 1947). C. Antenna of gallicola (after Annand 1928, modified). 


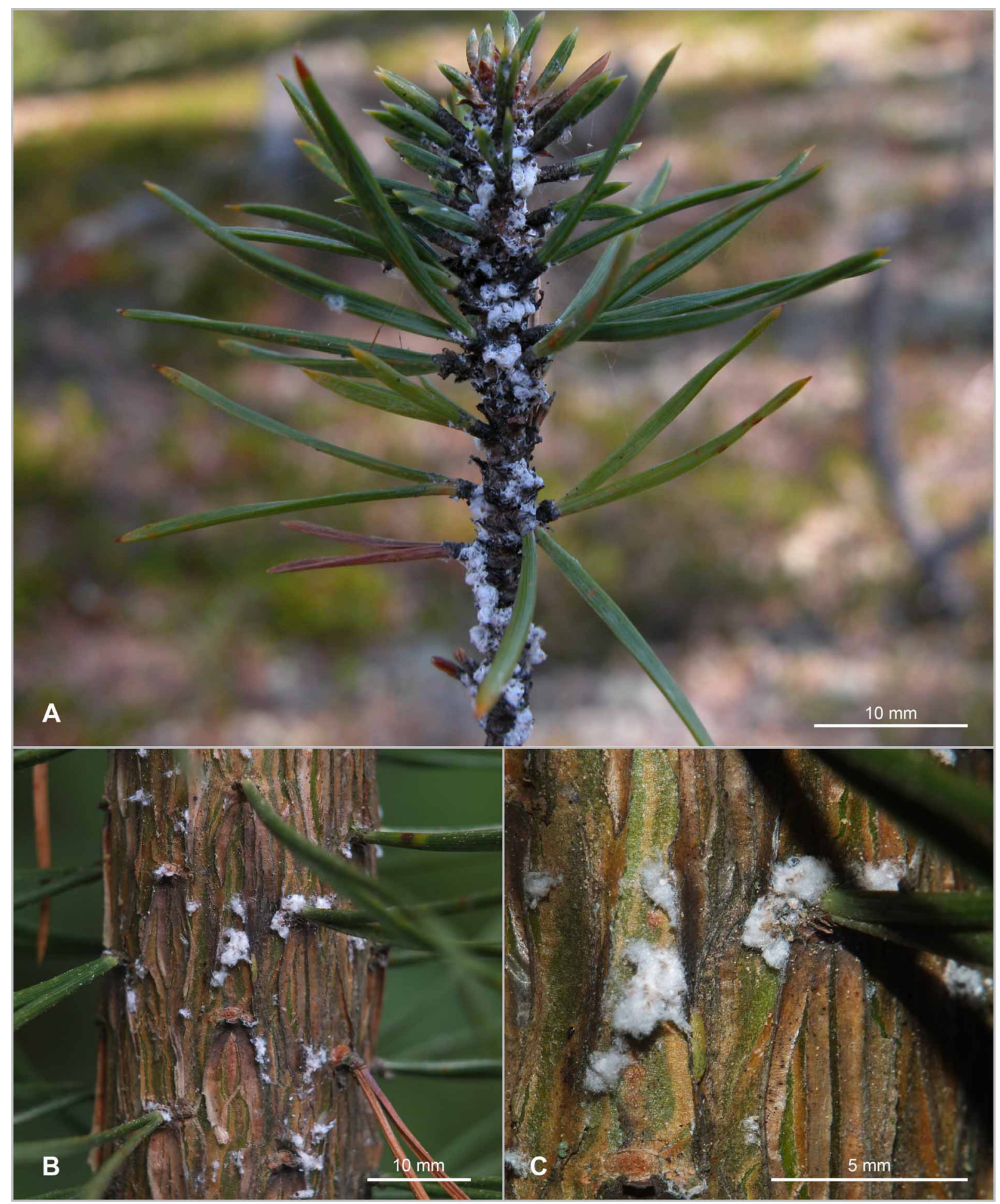

Fig. 72. Pineus (Pineus) pini (Macquart, 1819) on Pinus sylvestris. A. Old colony on dwarfish pine. B-C. Apterae on trunk of young tree. 


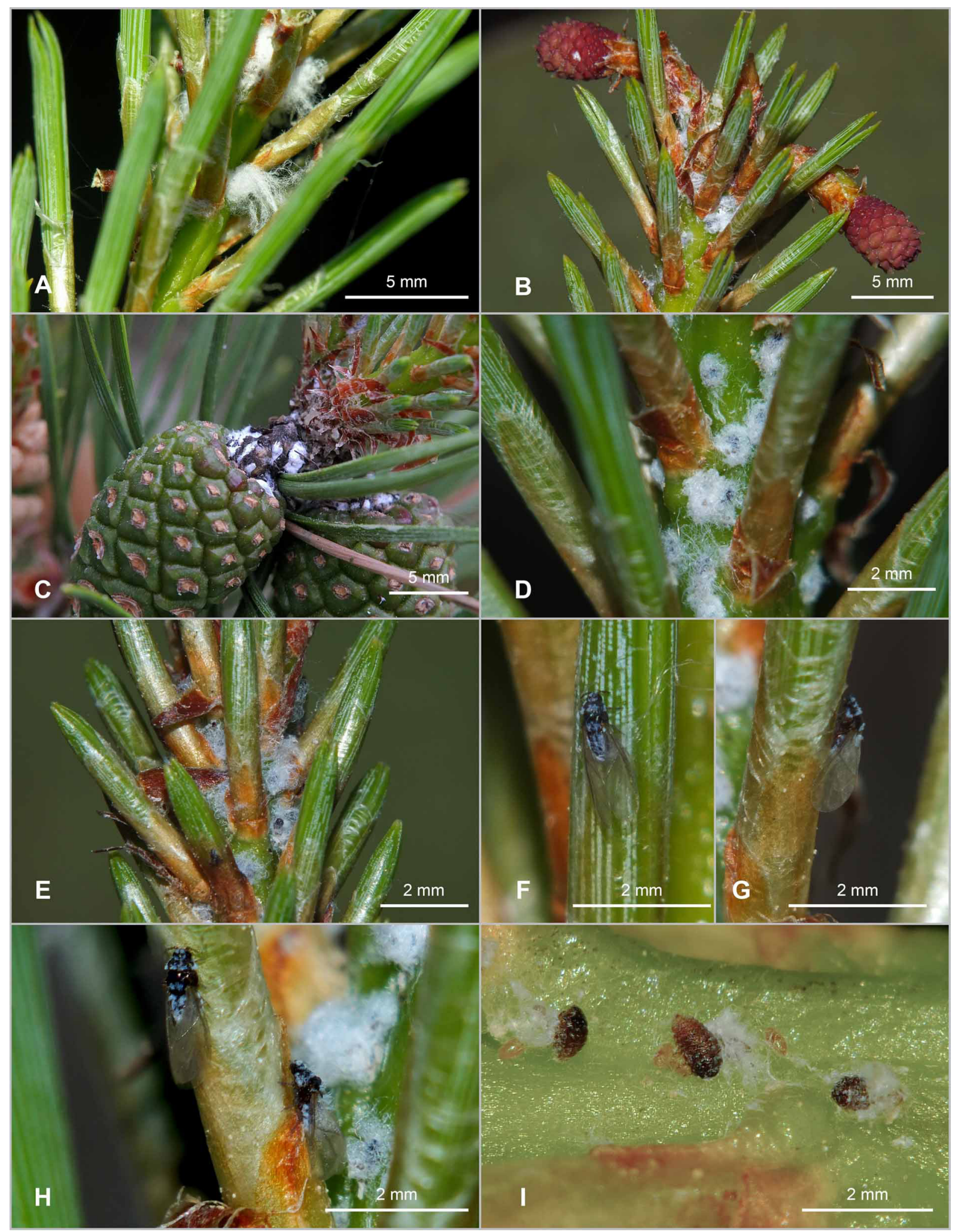

Fig. 73. Pineus (Pineus) pini (Macquart, 1819) on young growth of Pinus sylvestris. A. Apterae. B-D. Apterae and juvenile alatae. E-H. Alatae. I. Apterae with wax wool removed. 


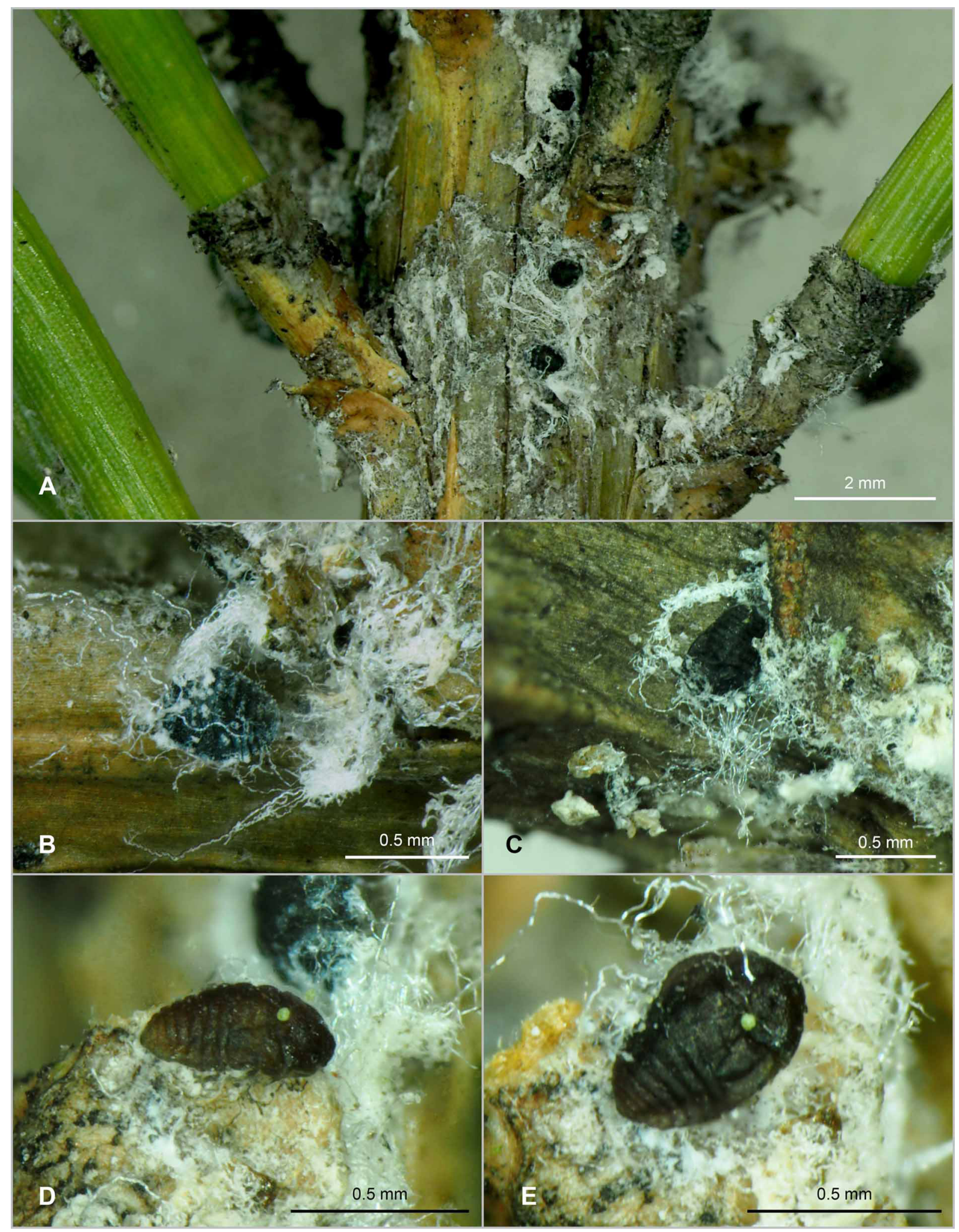

Fig. 74. Pineus (Pineus) pini (Macquart, 1819) on Pinus sylvestris in winter. A. Colony in top of dwarfish plant growing on exposed rock. B. Juvenile aptera. C-E. Juvenile alatae, excess wax wool and covering bark flakes removed. 


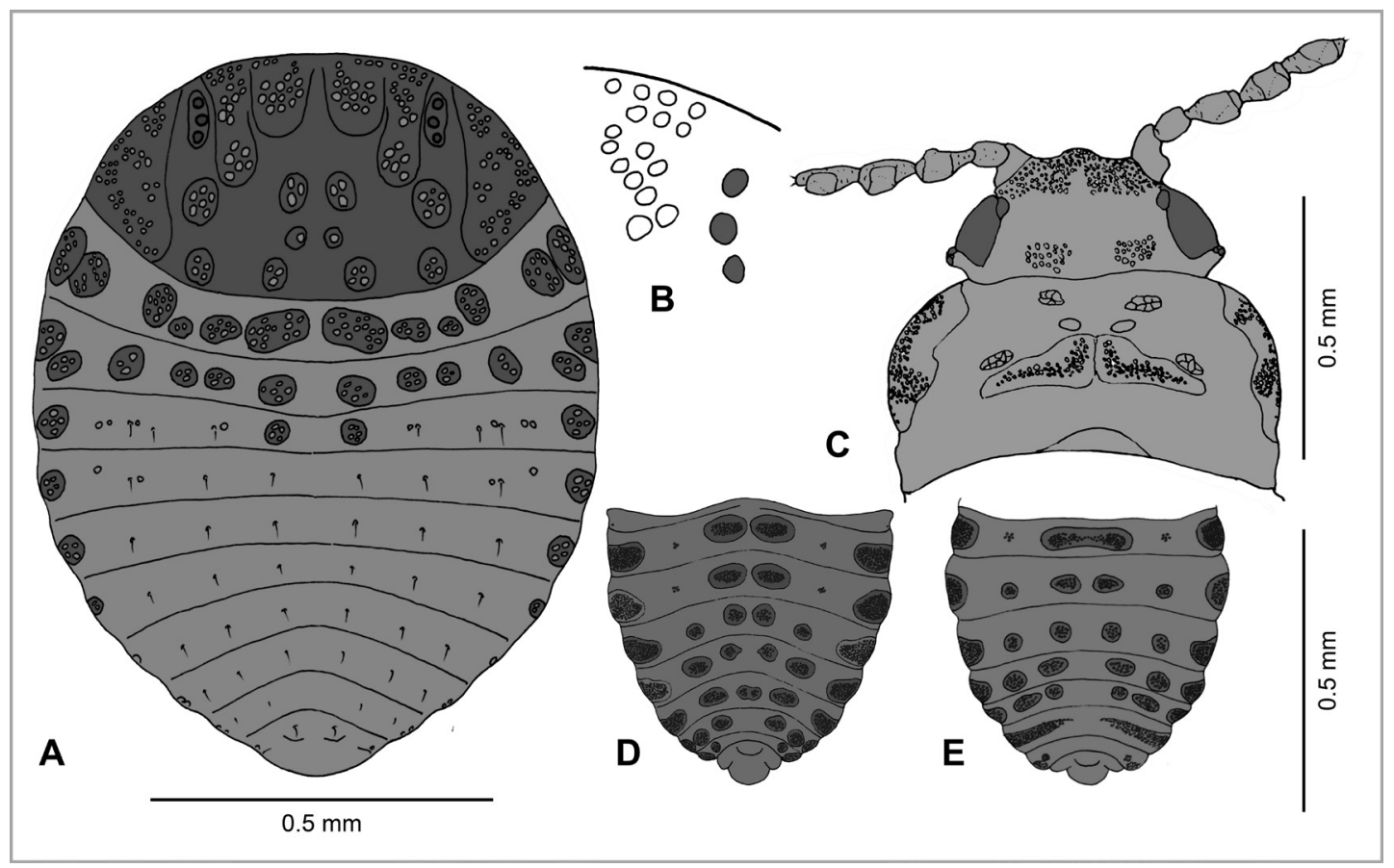

Fig. 75. Pineus (Pineus) pini (Macquart, 1819). A. Adult aptera (after Inouye 1953, modified from Pashchenko 1988). B. As A, showing outer intraocular group of wax gland pits. C. Adult alata, head and prothorax, showing arrangement of wax glands (after Carter 1971, modified from Heie 2004). D-E. Adult alata, abdomen (after Börner, modified from Heie 2004).

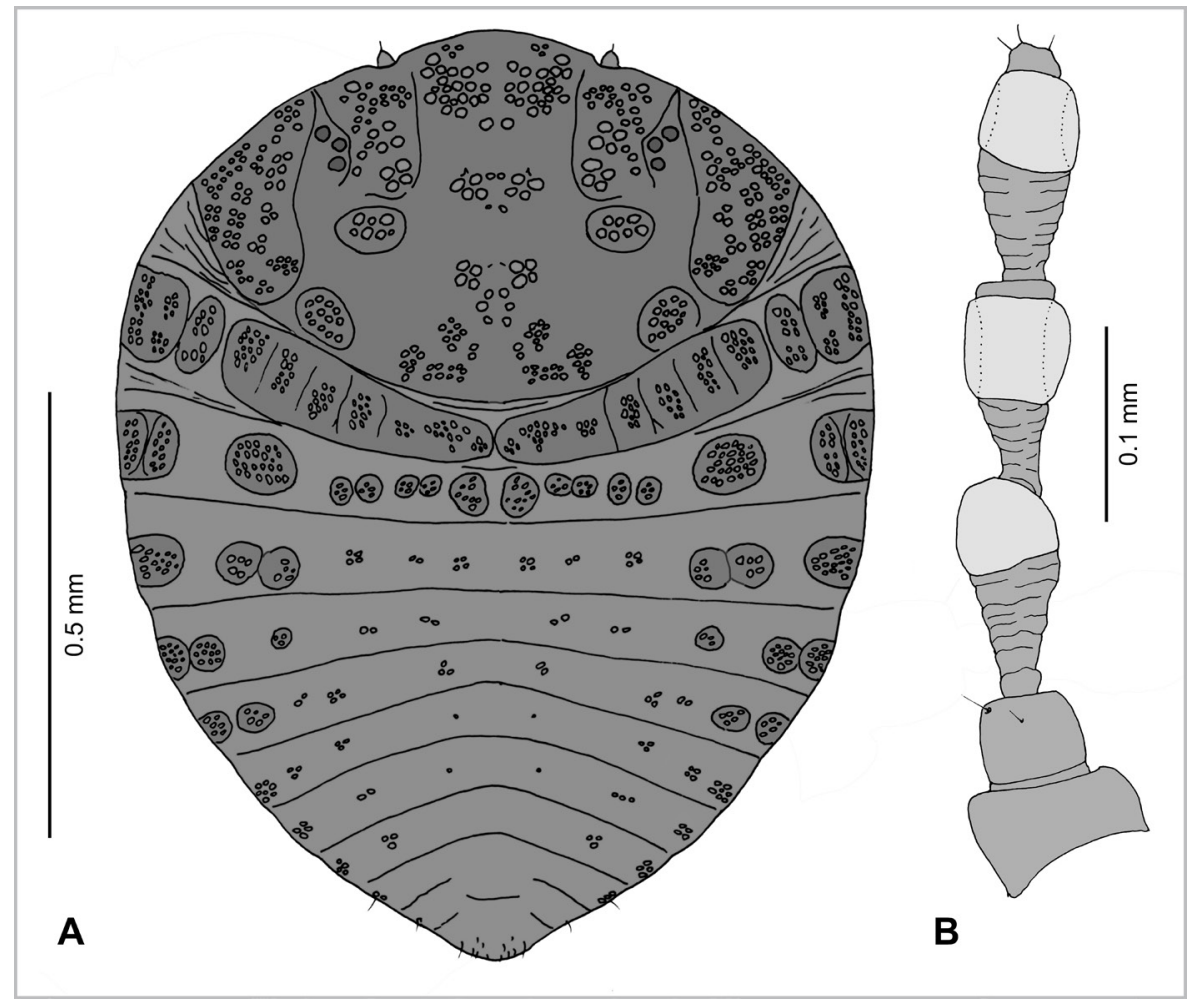

Fig. 76. Pineus (Pineus) orientalis (Dreyfus, 1889). A. Aptera (after Inouye 1953, modified from Pashchenko 1988). B. Antenna of gallicola (after Annand 1928, modified). 


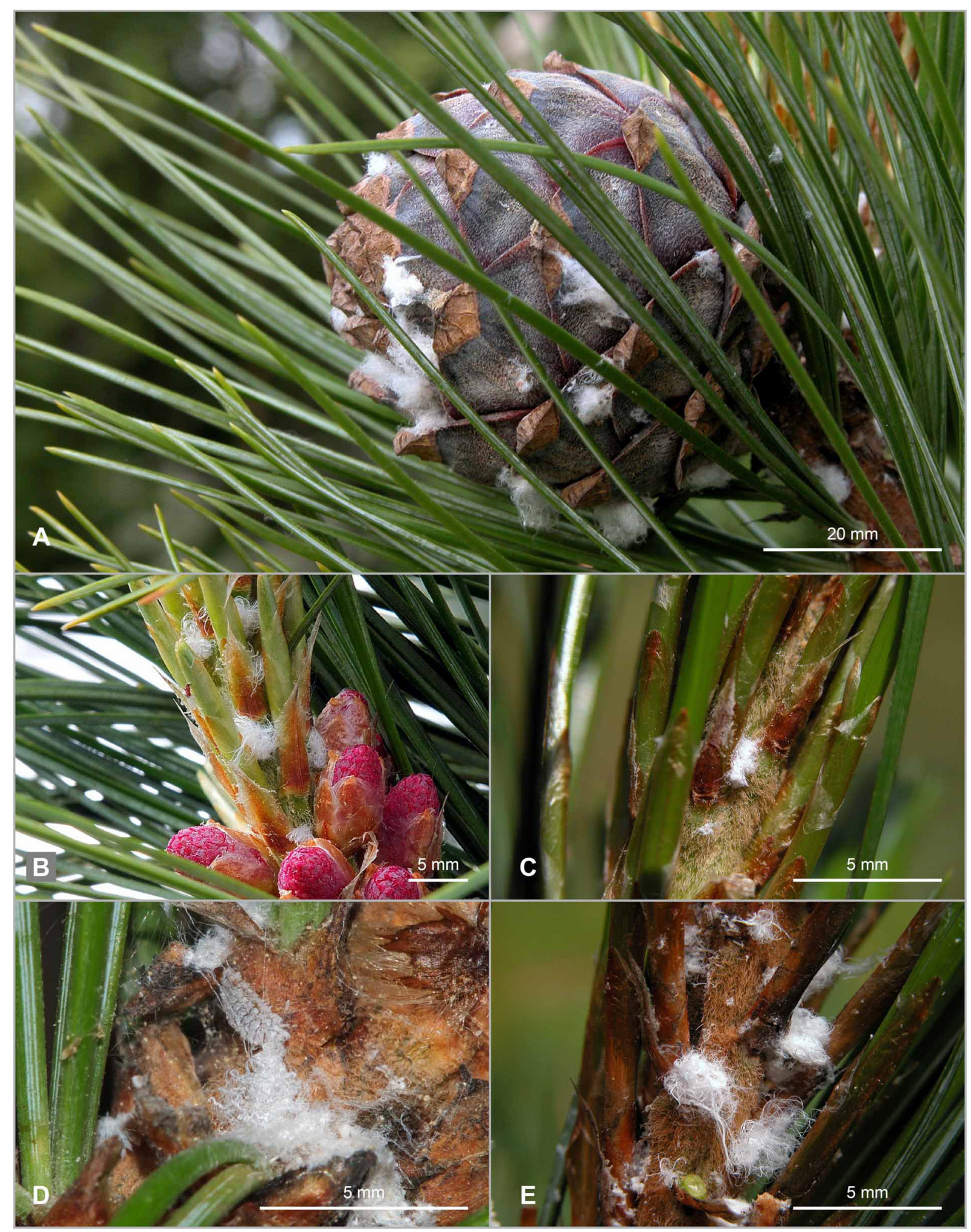

Fig. 77. Pineus (Pineus) cembrae (Cholodkovsky, 1888). Apterae on young growth of Pinus cembra. In $\mathrm{D}$, also a scymnine ladybird larva (Coleoptera, Coccinellidae). 


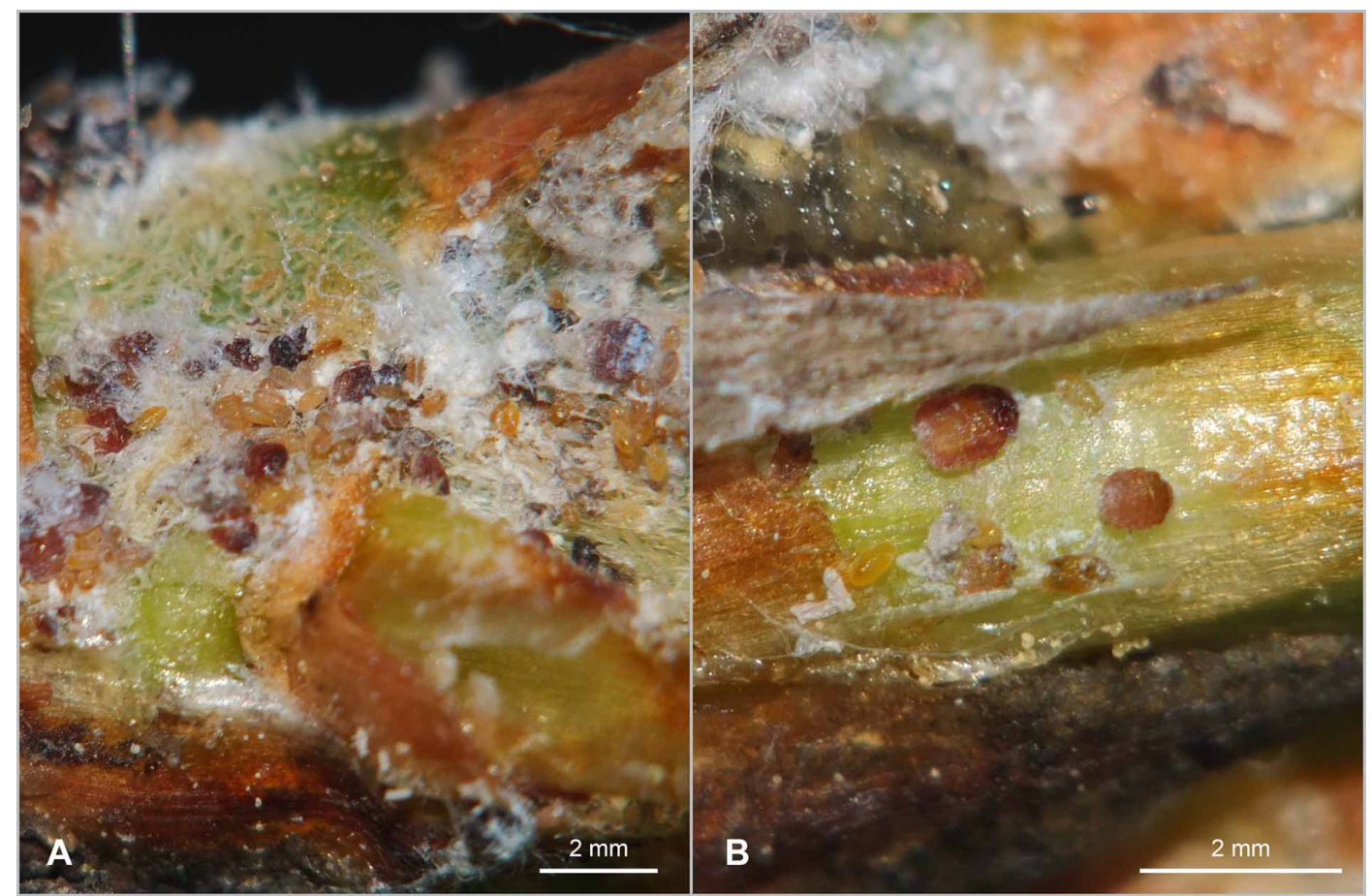

Fig. 78. Pineus (Pineus) cembrae (Cholodkovsky, 1888). Apterae with eggs and juveniles on young growth of Pinus cembra. (wax wool removed). In B, also a syrphid (Diptera, Syrphidae) larva.

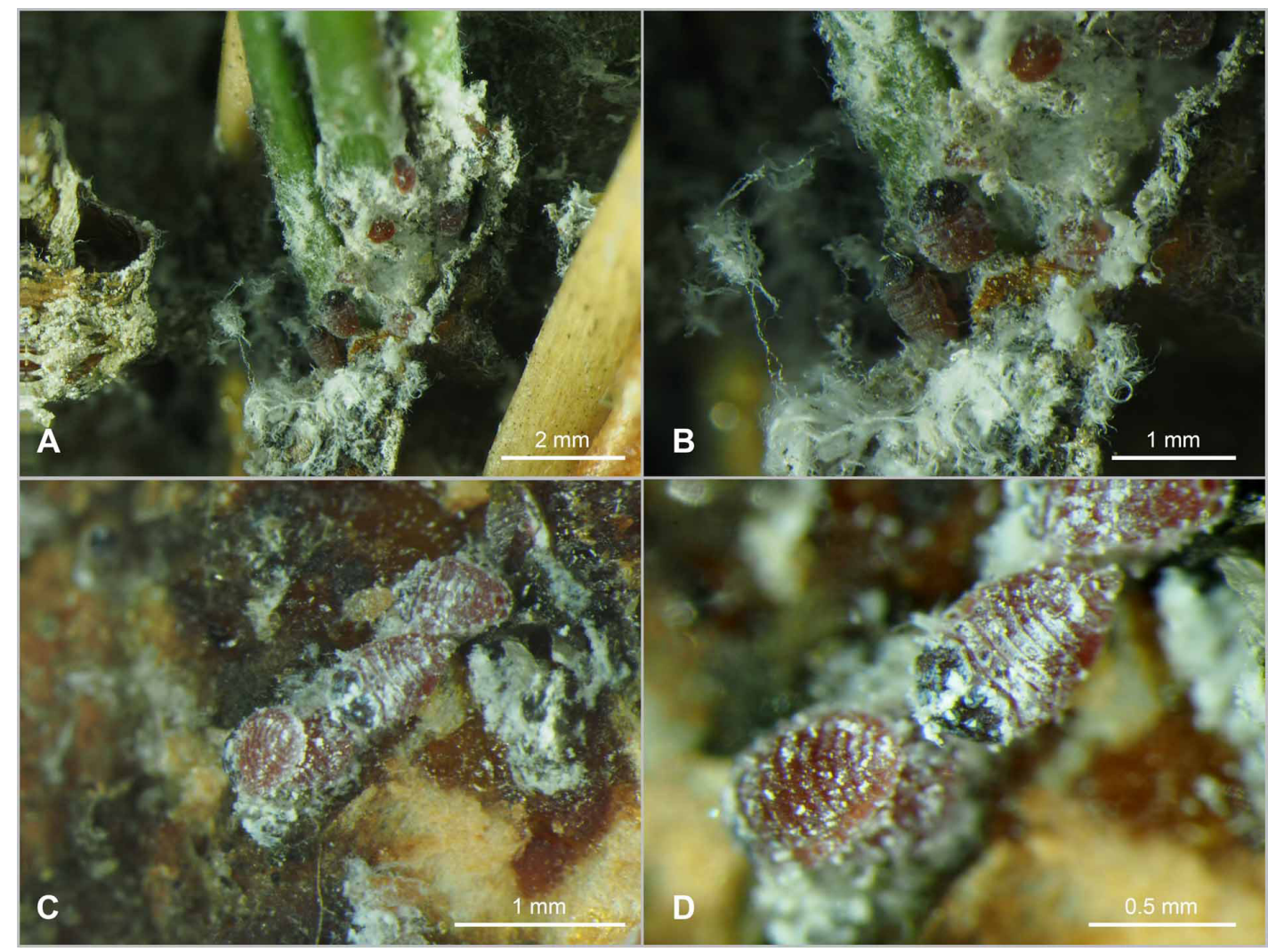

Fig. 79. Pineus (Pineus) cembrae (Cholodkovsky, 1888). Overwintering apterae on twig of Pinus cembra. Bud scales and wax wool removed. 


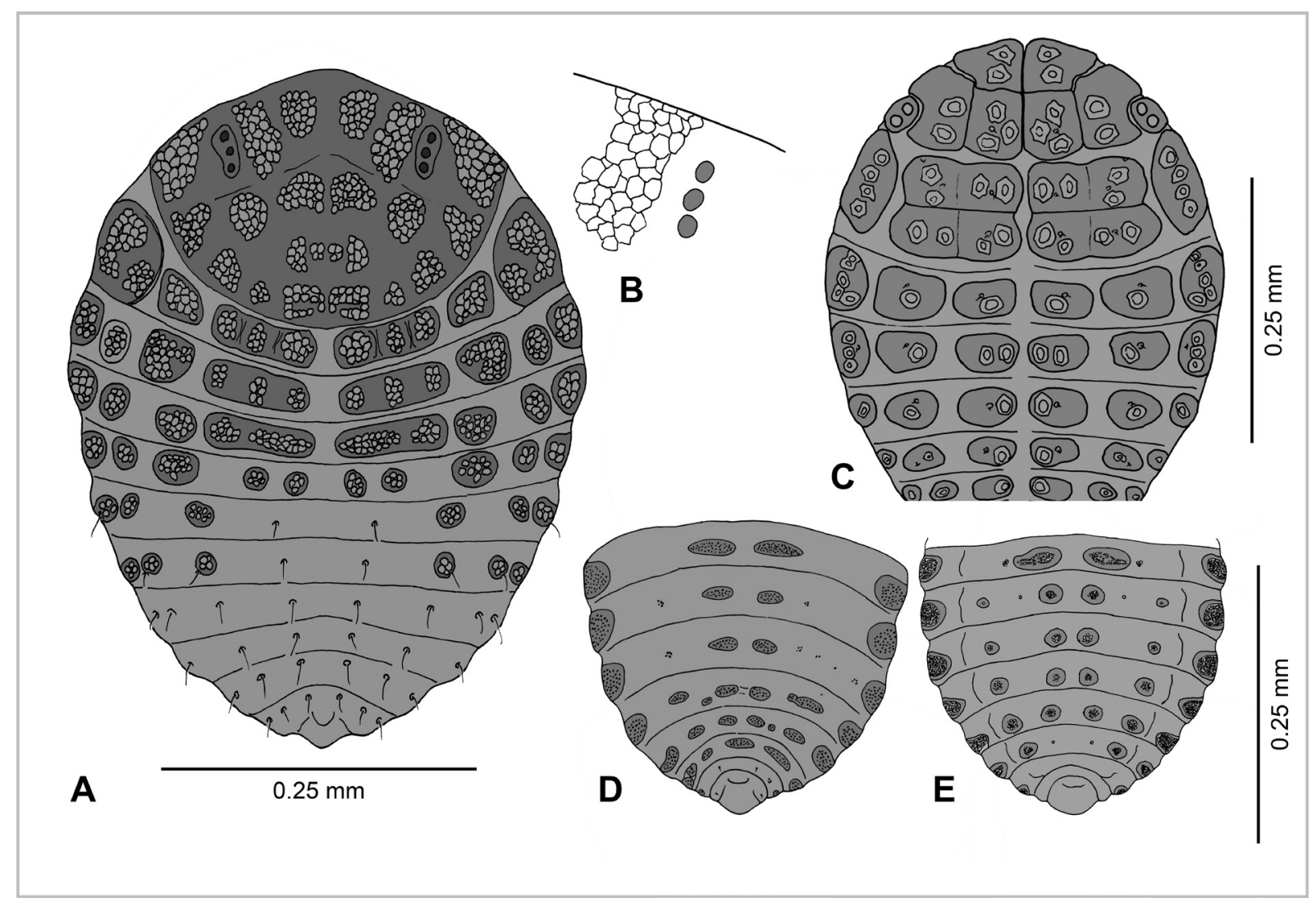

Fig. 80. Pineus (Pineus) cembrae (Cholodkovsky, 1888). A-B. Adult aptera; in B showing outer intraocular group of wax gland pits (after Inouye 1953, modified.). C. Hibernating nymph (after Inouye 1953, modified from Pashchenko 1988). D-E. Abdomen of gallicola (D) and sexupara (E) (after Börner, modified from Heie 2004).

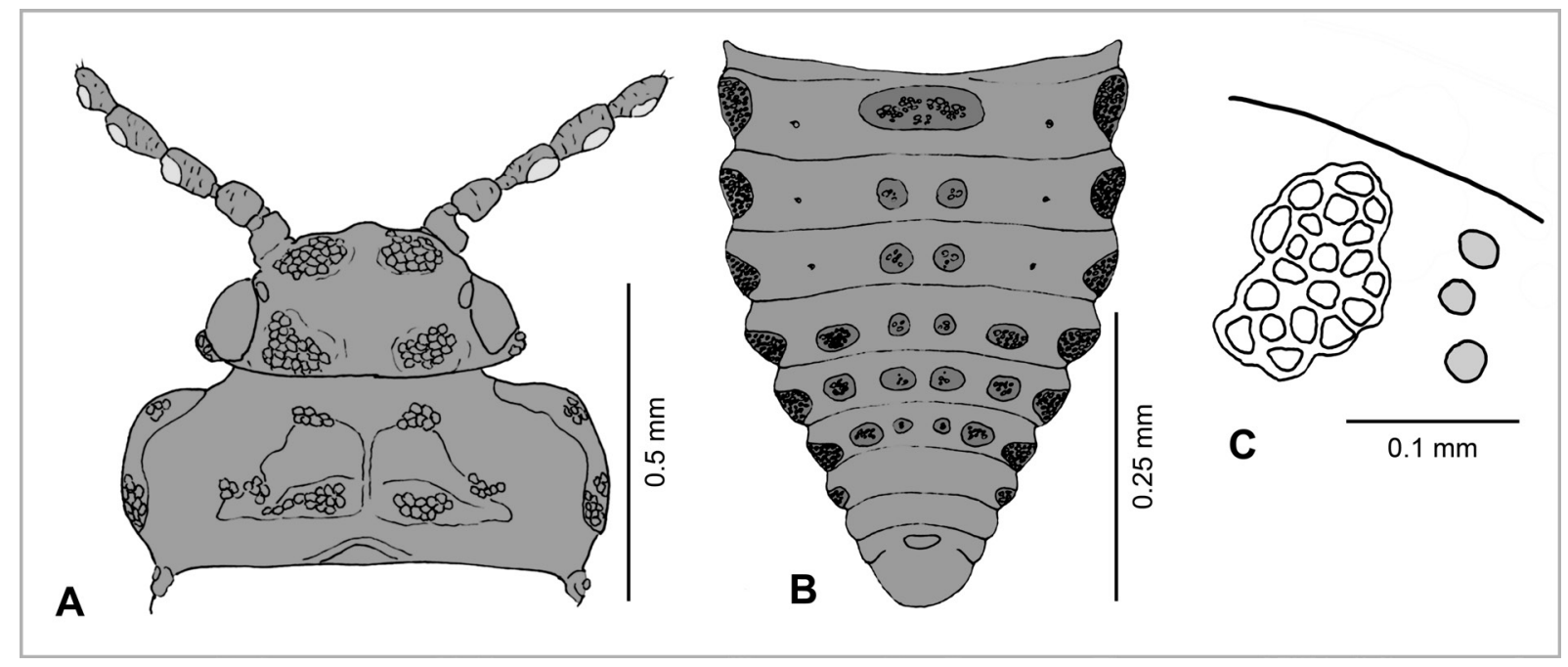

Fig. 81. Pineus (Pineus) strobi (Hartig, 1837). A. Alata, head and prothorax (after Carter 1971, modified from Heie 2004). B. Alata, abdomen (after Börner, modified from Heie 2004). C. Aptera, outer intraocular group of wax gland pits (freely after Blackman \& Eastop 1994). 


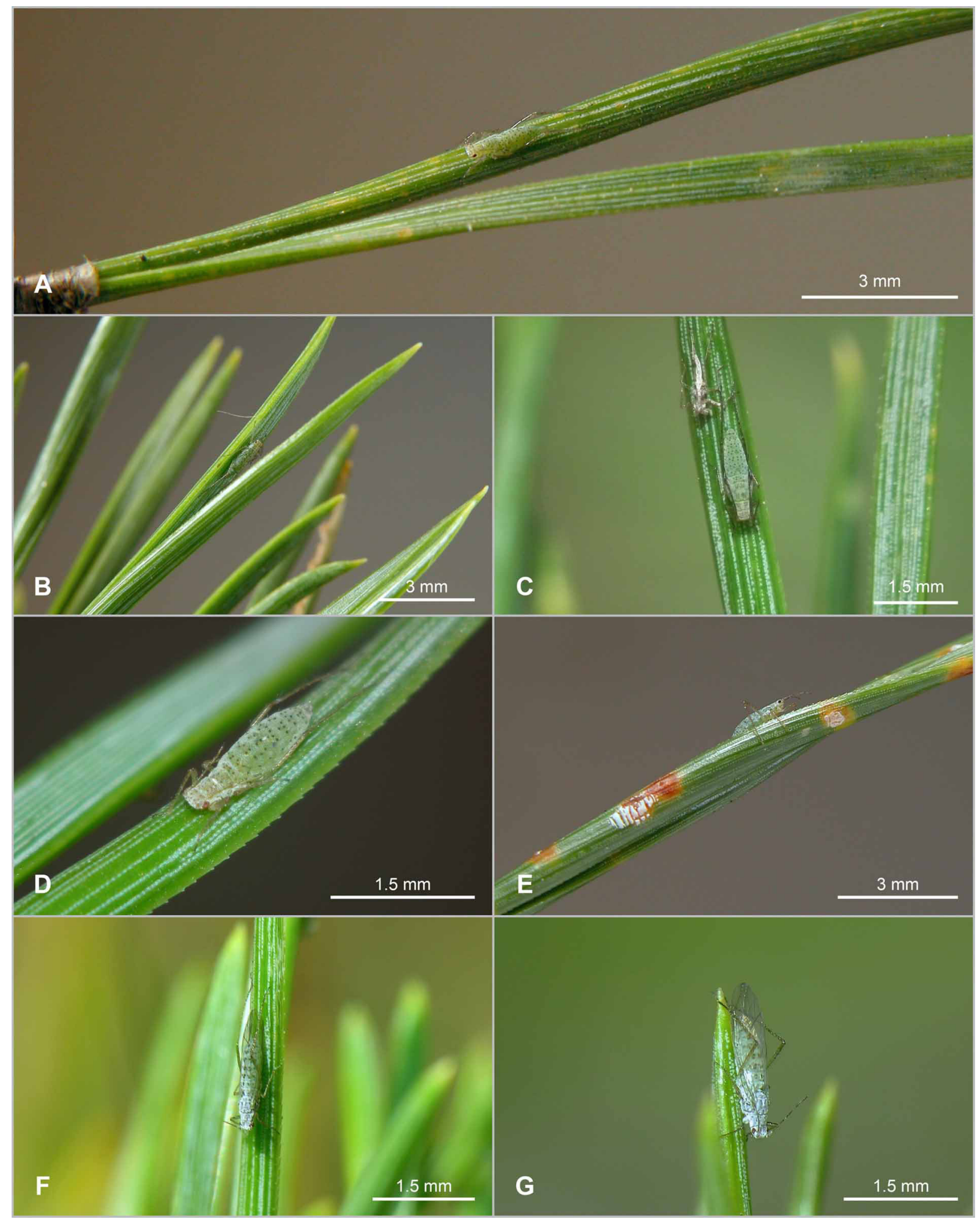

Fig. 82. Eulachnus agilis (Kaltenbach, 1843) on needles of Pinus sylvestris. A-E. Apterae. F-G. Alatae. 


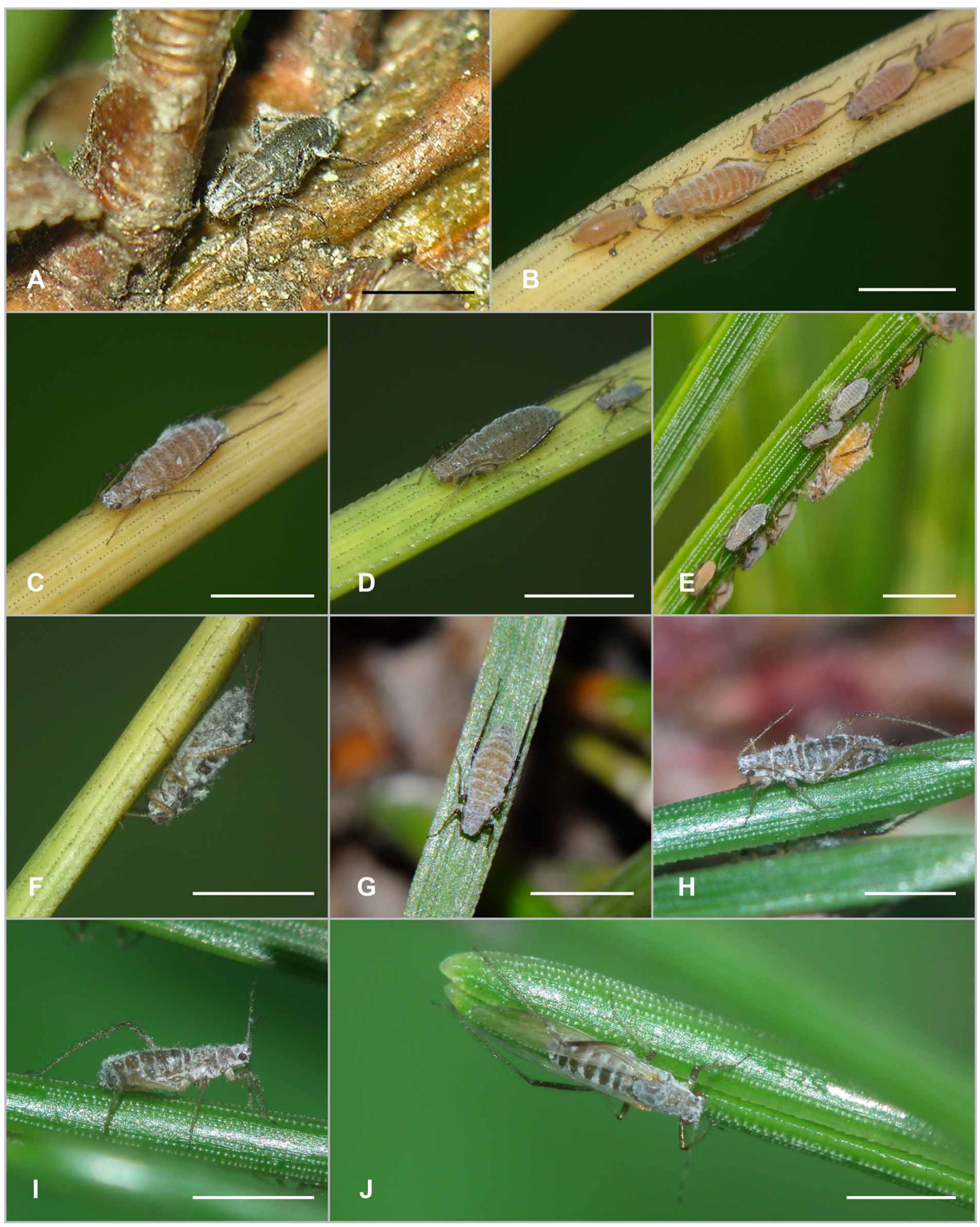

Fig. 83. Eulachnus rileyi (Williams, 1911) on Pinus mugo. Apterae, juveniles, and one alata (J). Scale bars: $2 \mathrm{~mm}$. 


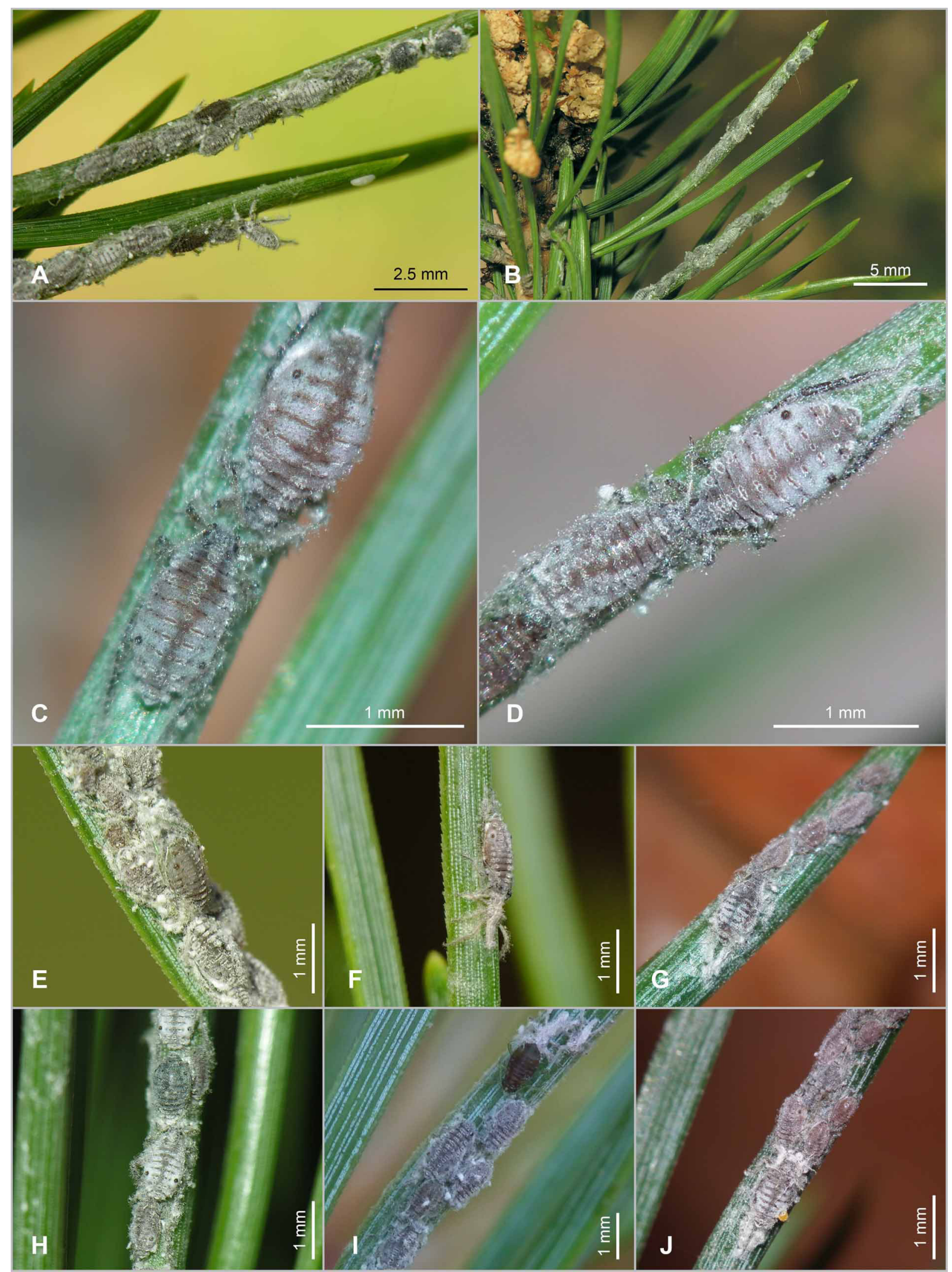

Fig. 84. Schizolachnus pineti (Fabricius, 1781) on Pinus sylvestris. The black individuals are newly molted, still without wax cover. 


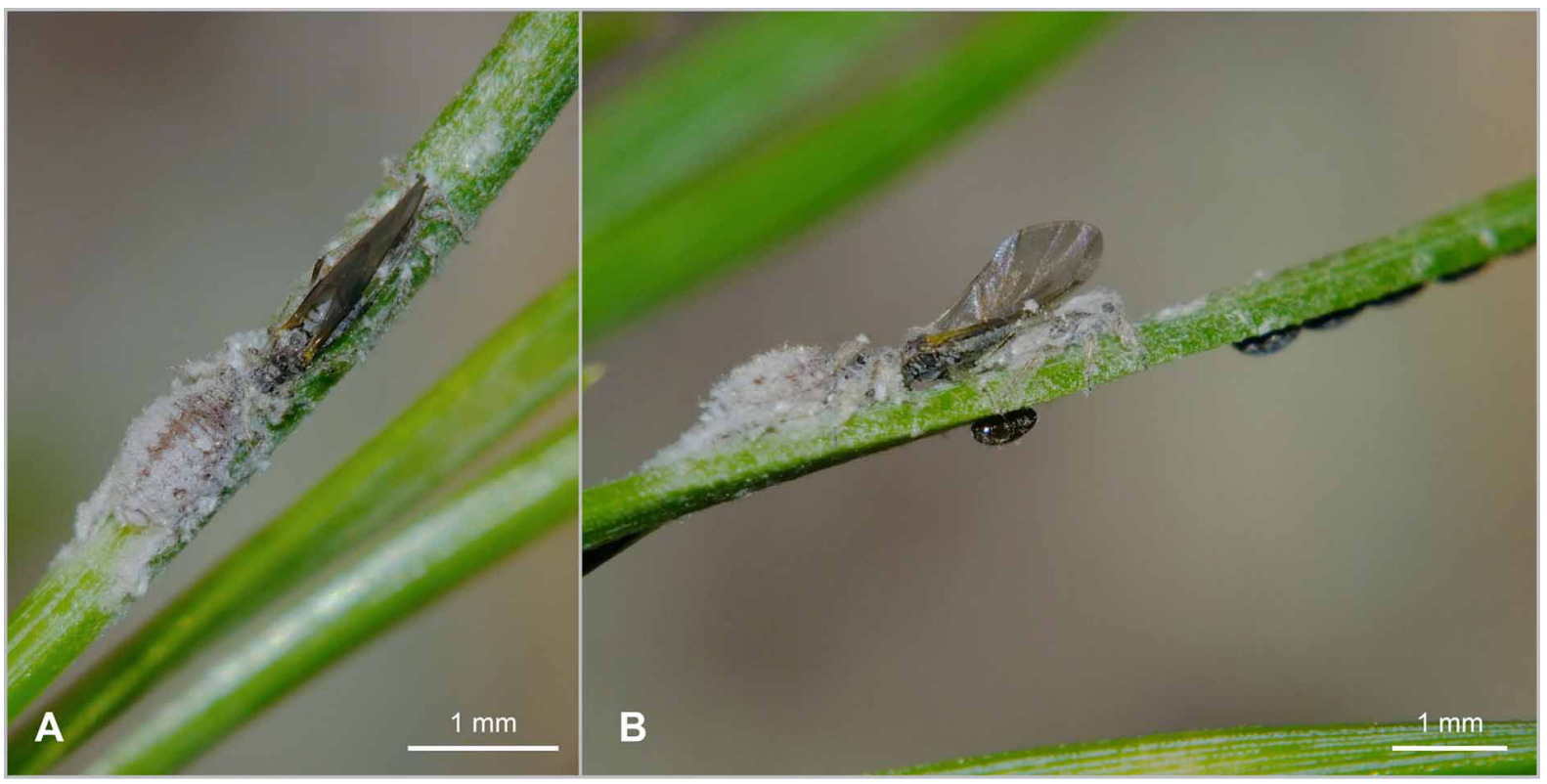

Fig. 85. Schizolachnus pineti (Fabricius, 1781). Aptera, alata, and eggs (in B) on needles of Pinus sylvestris.

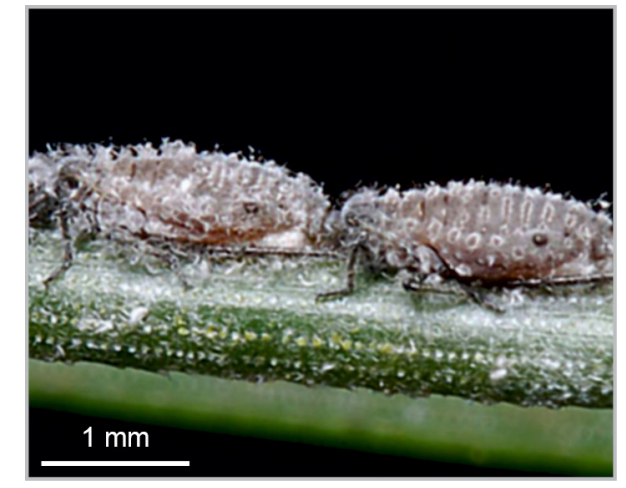

Fig. 86. Schizolachnus obscurus Börner, 1940. Aptera on Pinus nigra (from Dransfield \& Brightwell 2015, licensed under Creative Commons Attribution 3.0, downloaded 2 Mar. 2016).

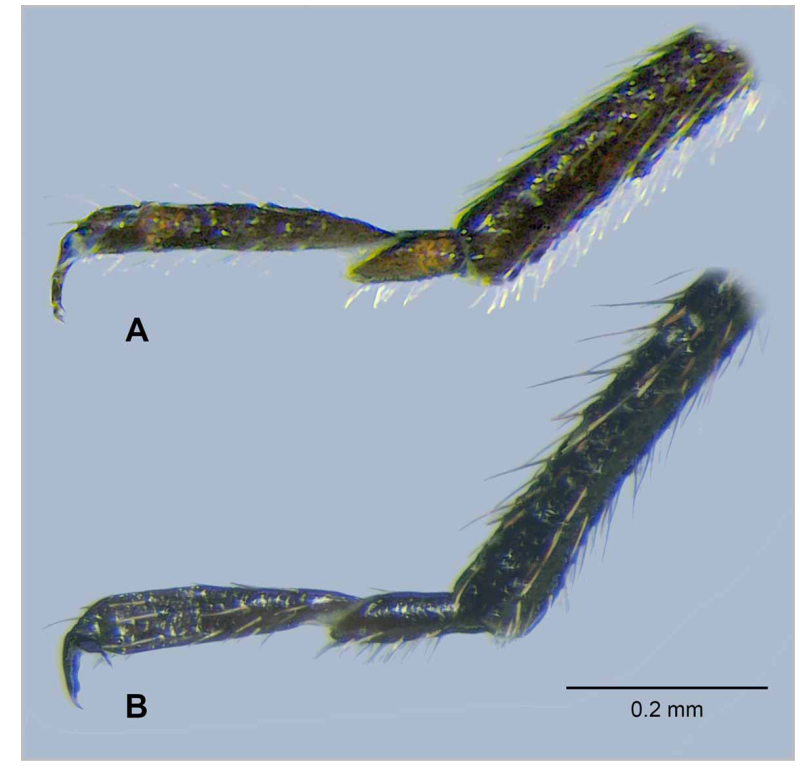

Fig. 87. Hind tarsus, showing length and form of second segment. A. Cinara (Cinara) nuda Mordvilko, 1895 B. C. pinea (Mordvilko, 1895). 


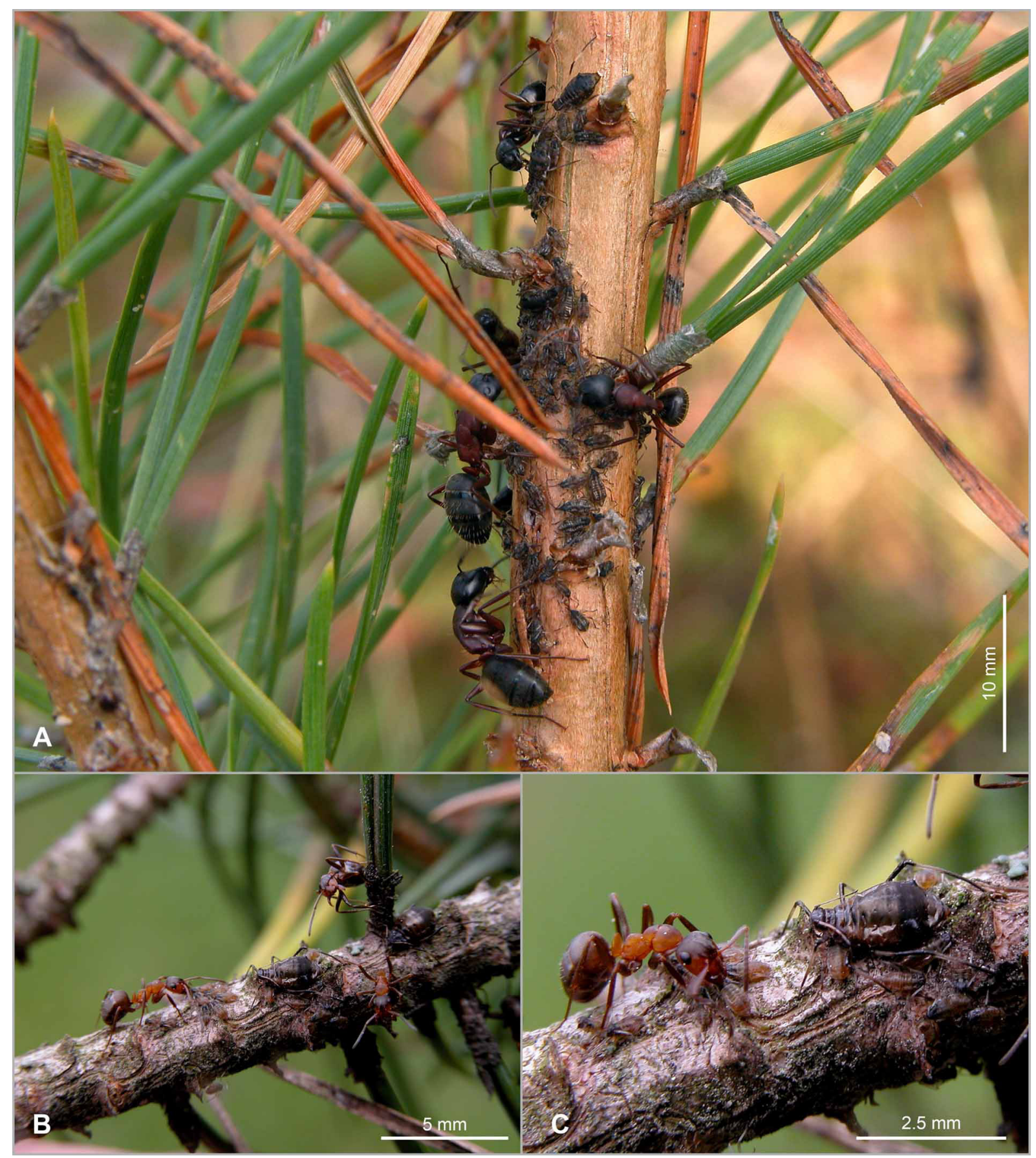

Fig. 88. Cinara (Cinara) pini (Linnaeus, 1758) on Pinus sylvestris. A. Colony on previous year's terminal shoot, attended by Camponotus herculeanus (Linnaeus, 1758). B-C. Apterae with young on a 2 year-old twig, attended by Formica polyctena Förster, 1850. 


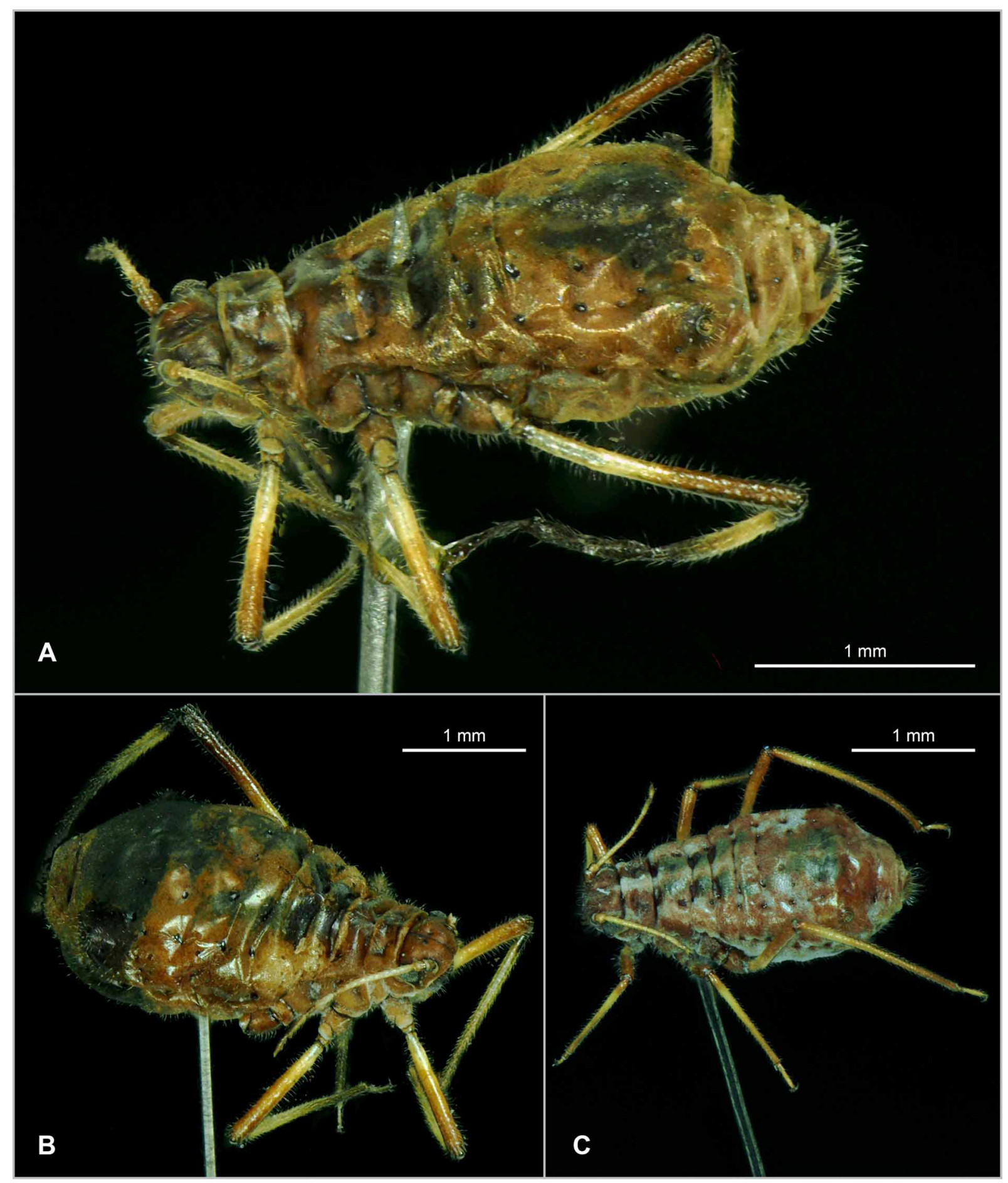

Fig. 89. Cinara (Cinara) pini (Linnaeus, 1758). Freeze-dried apterae. A-B. Adults. C. Full-grown juvenile. 


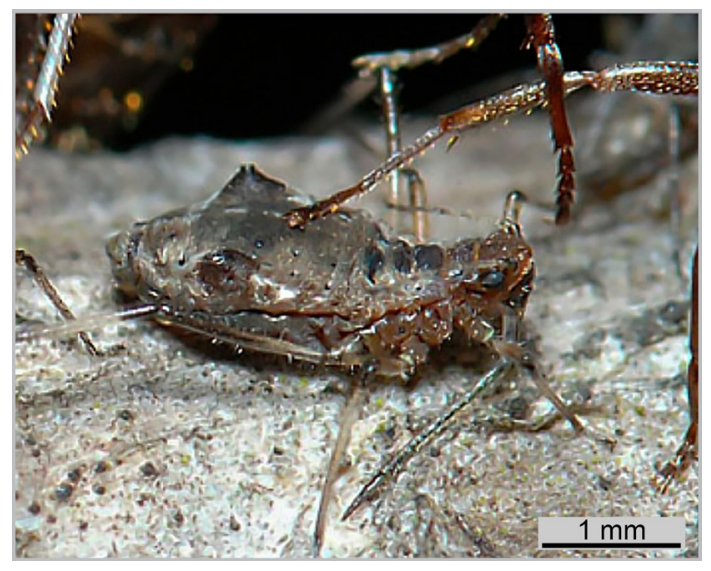

Fig. 90. Cinara (Cinara) acutirostris Hille Ris Lambers, 1956. Aptera on Pinus nigra (from Dransfield \& Brightwell 2015, licensed under Creative Commons Attribution 3.0, downloaded 2 Mar. 2016).

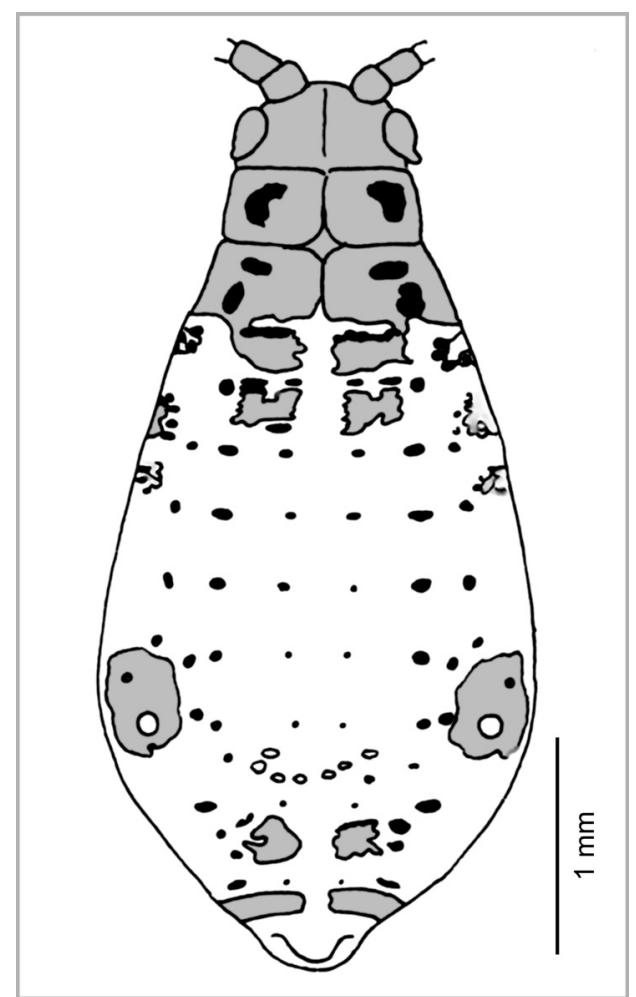

Fig. 91. Cinara (Cinara) cembrae (Seitner, 1936). Head, thorax and abdomen (after Pintera 1966, redrawn). 


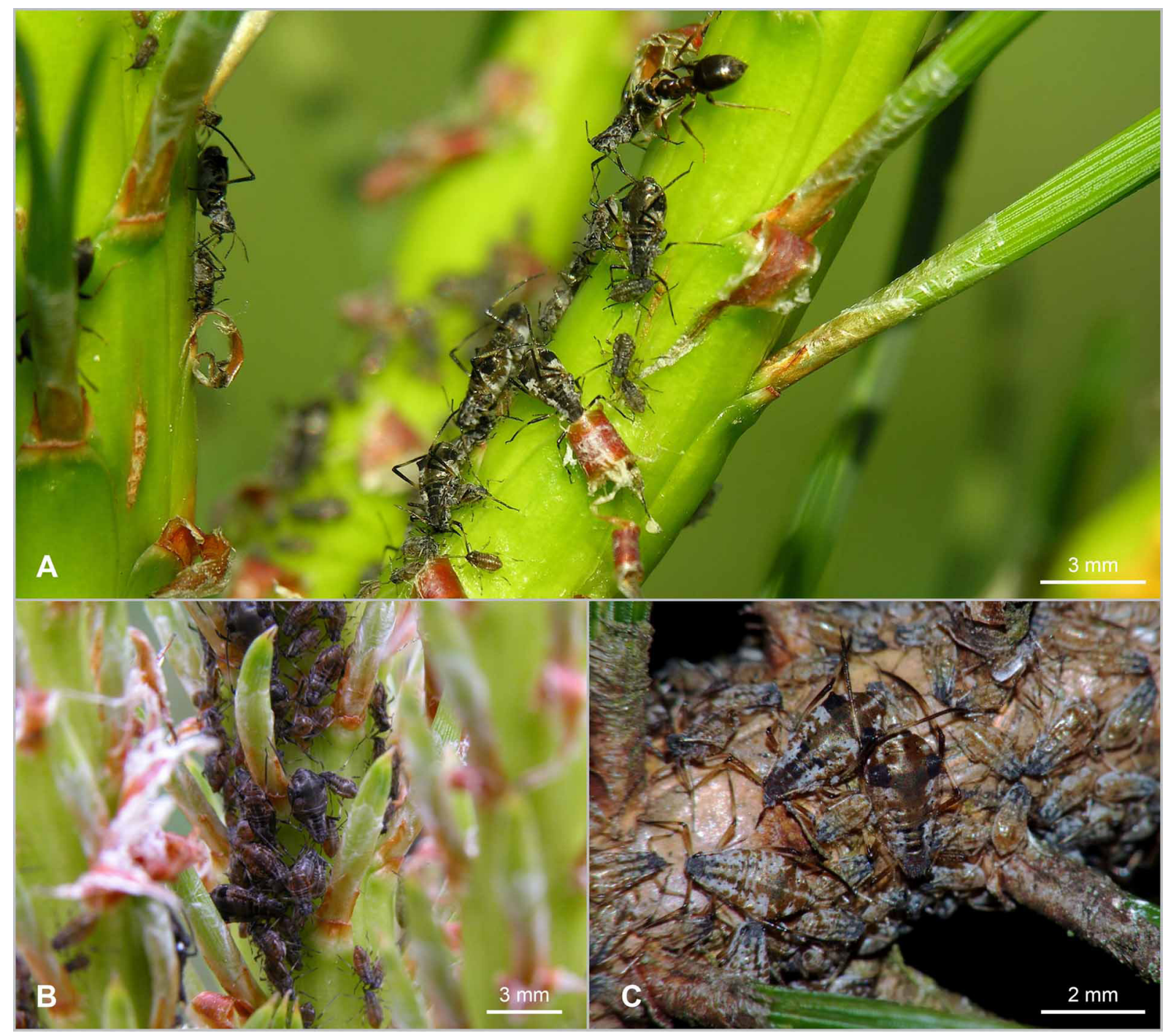

Fig. 92. Cinara (Cinara) nigritergi Mamontova-Solukha, 2002. Apterae and juveniles on Pinus sylvestris. A-B. Colonies on young shoot, attended by Lasius niger (Linnaeus, 1758). C. Colony on 2-3 year-old twig. 


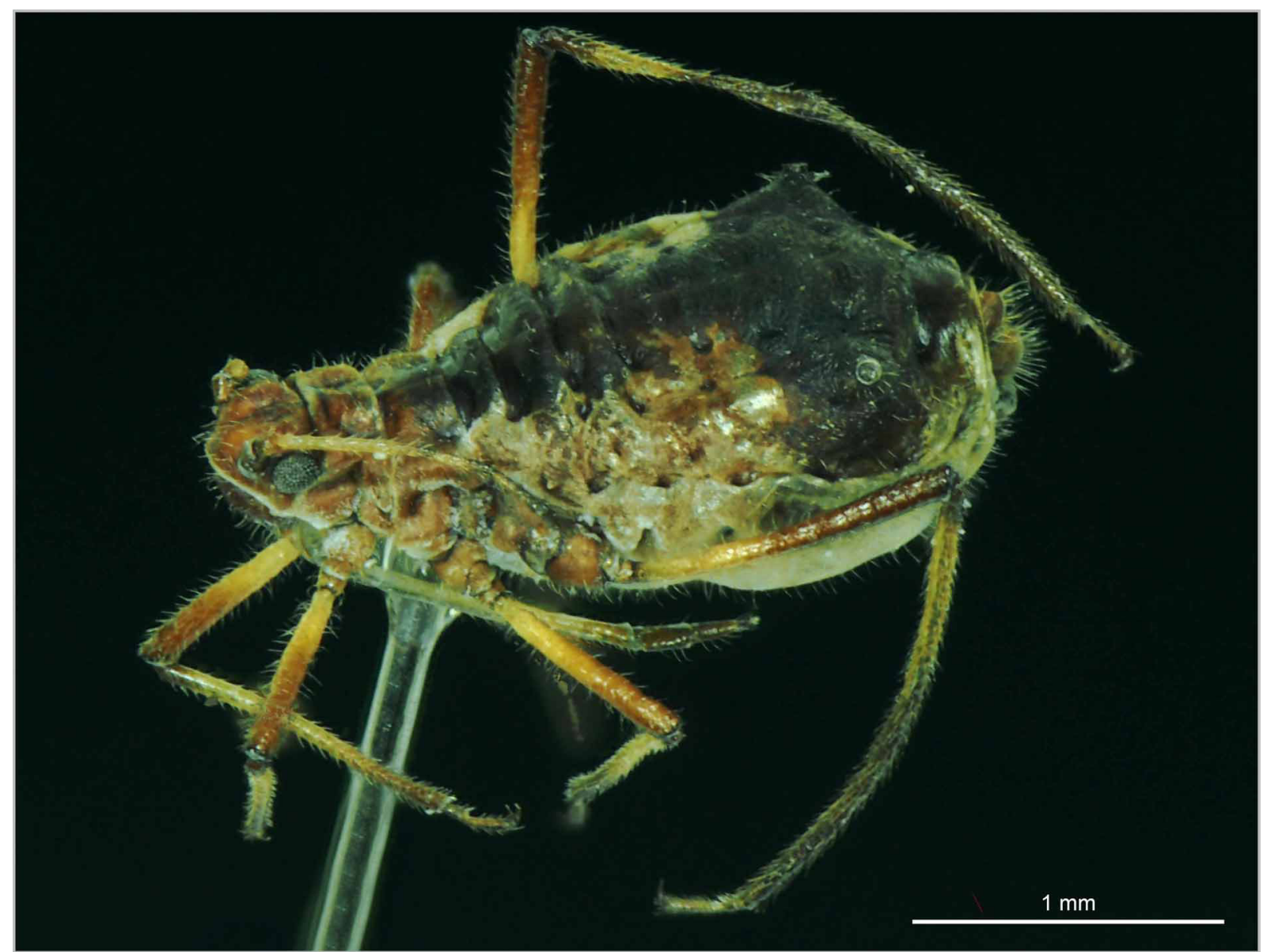

Fig. 93. Cinara (Cinara) nigritergi Mamontova-Solukha, 2002. Aptera (freeze-dried specimen). 


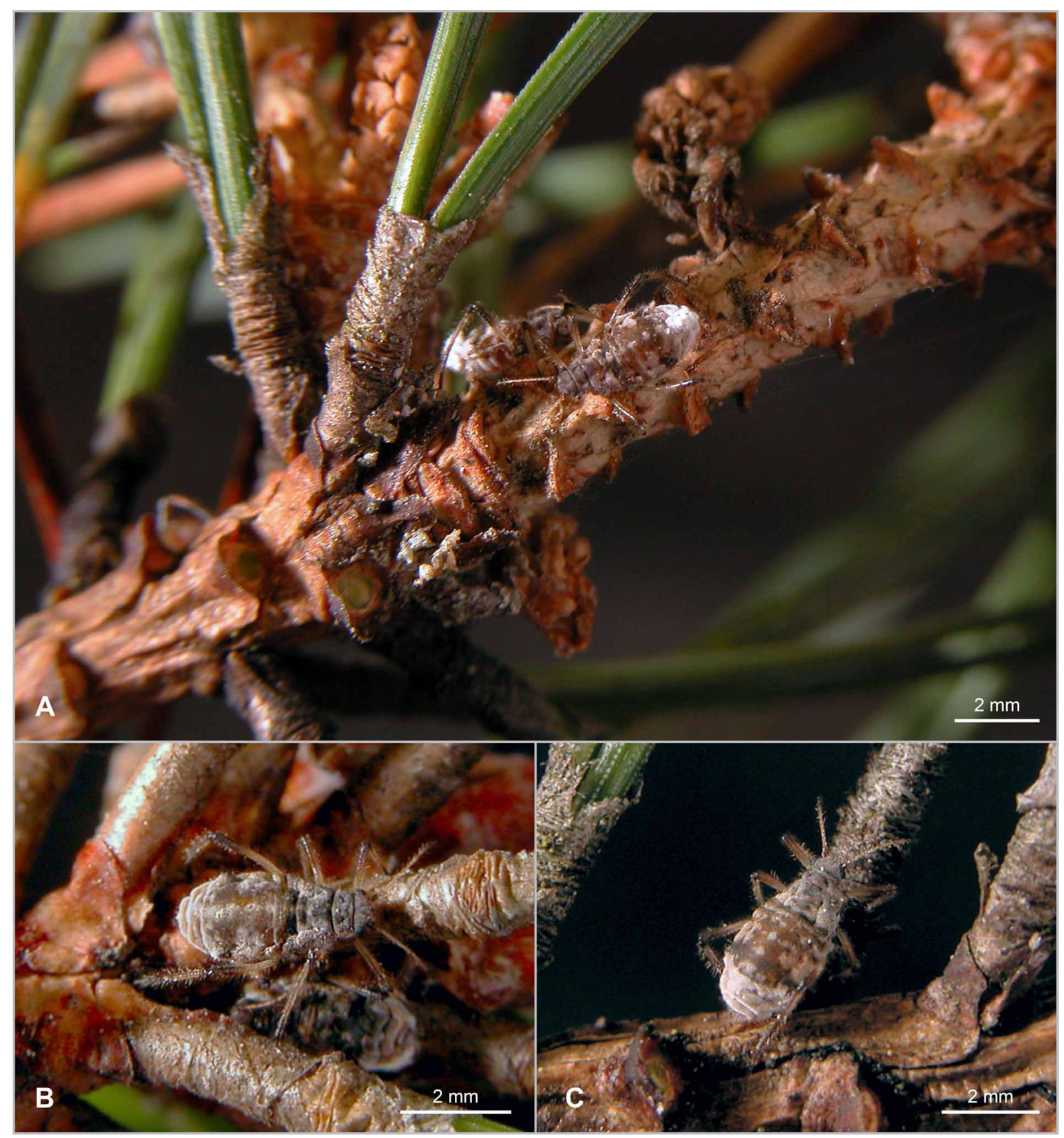

Fig. 94. Cinara (Cinara) pinihabitans (Mordvilko, 1895). Oviparae on Pinus sylvestris. 


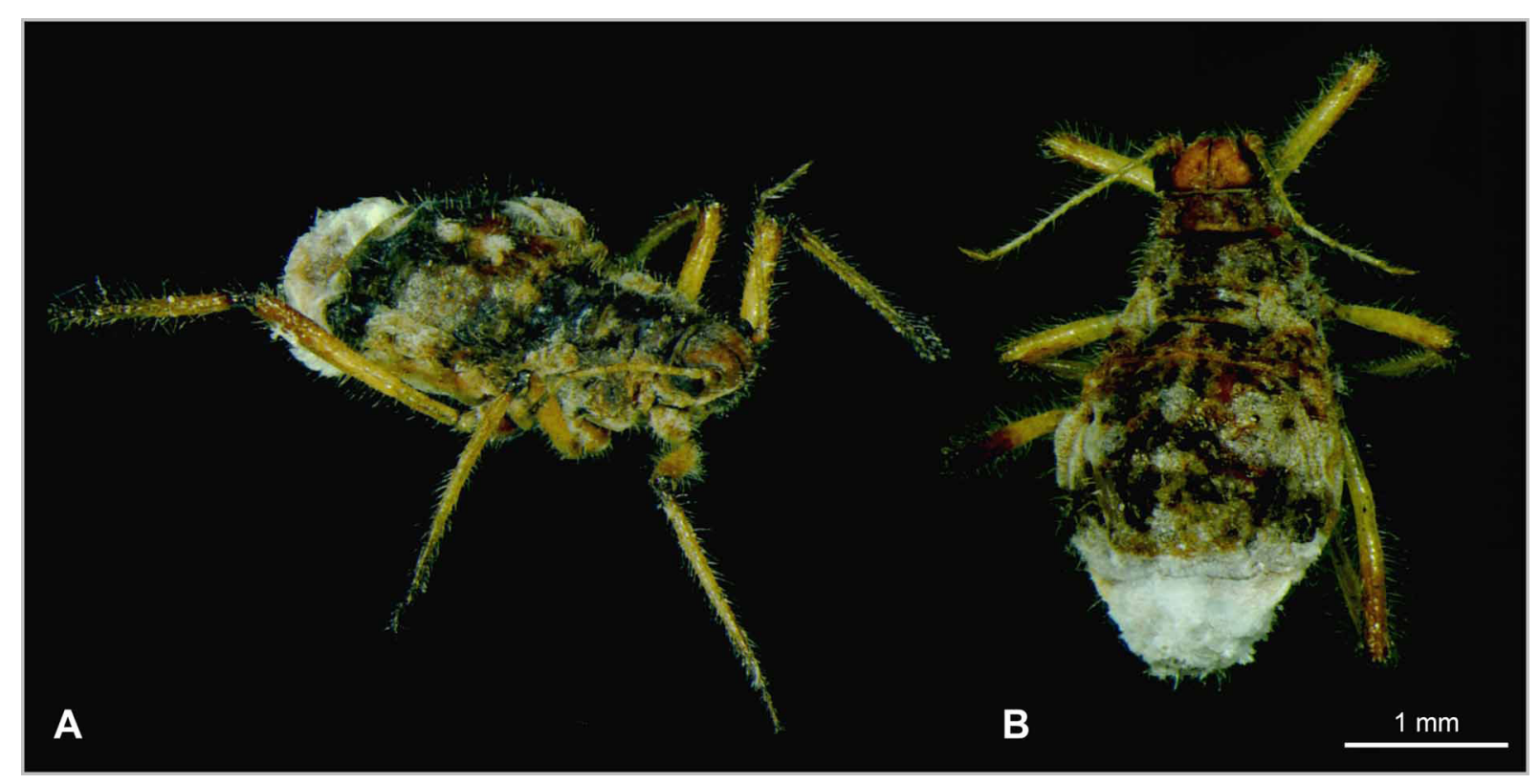

Fig. 95. Cinara (Cinara) pinihabitans (Mordvilko, 1895). Ovipara (freeze-dried specimens). 


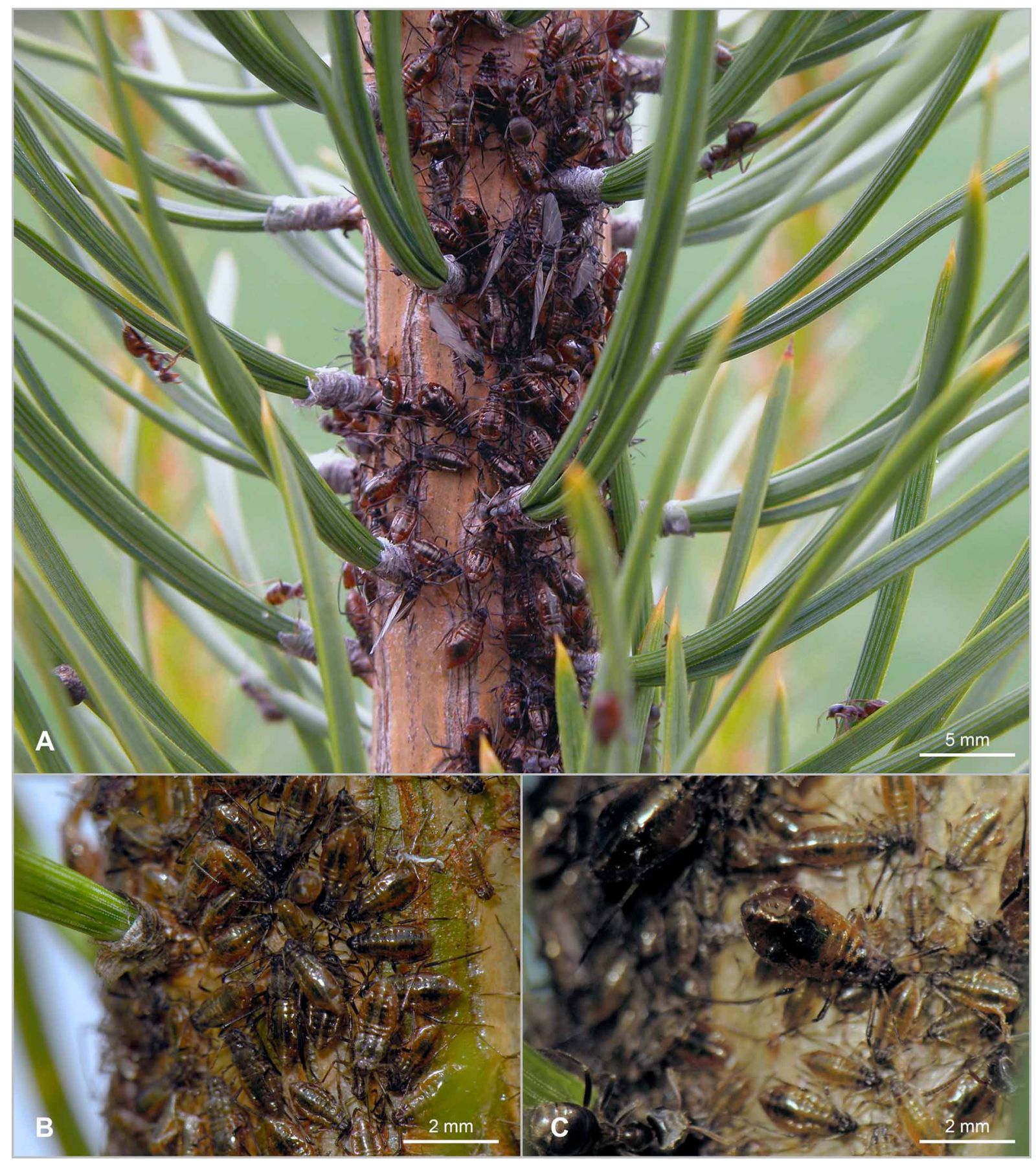

Fig. 96. Cinara (Cinara) nuda Mordvilko, 1895. Colony on trunk of young Pinus sylvestris, attended by Lasius niger (Linnaeus, 1758). 


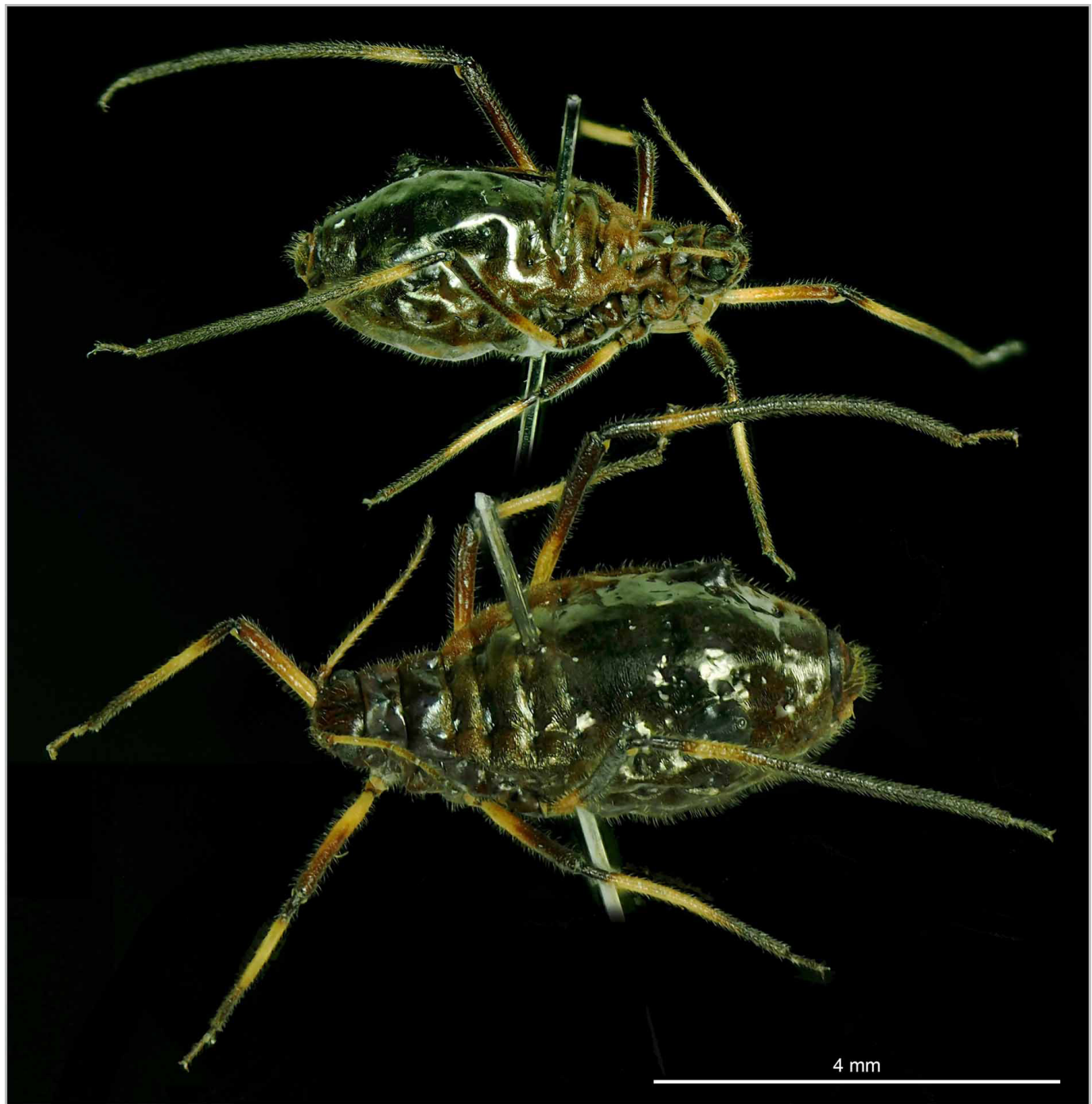

Fig. 97. Cinara (Cinara) nuda Mordvilko, 1895. Apterae (freeze-dried specimens).

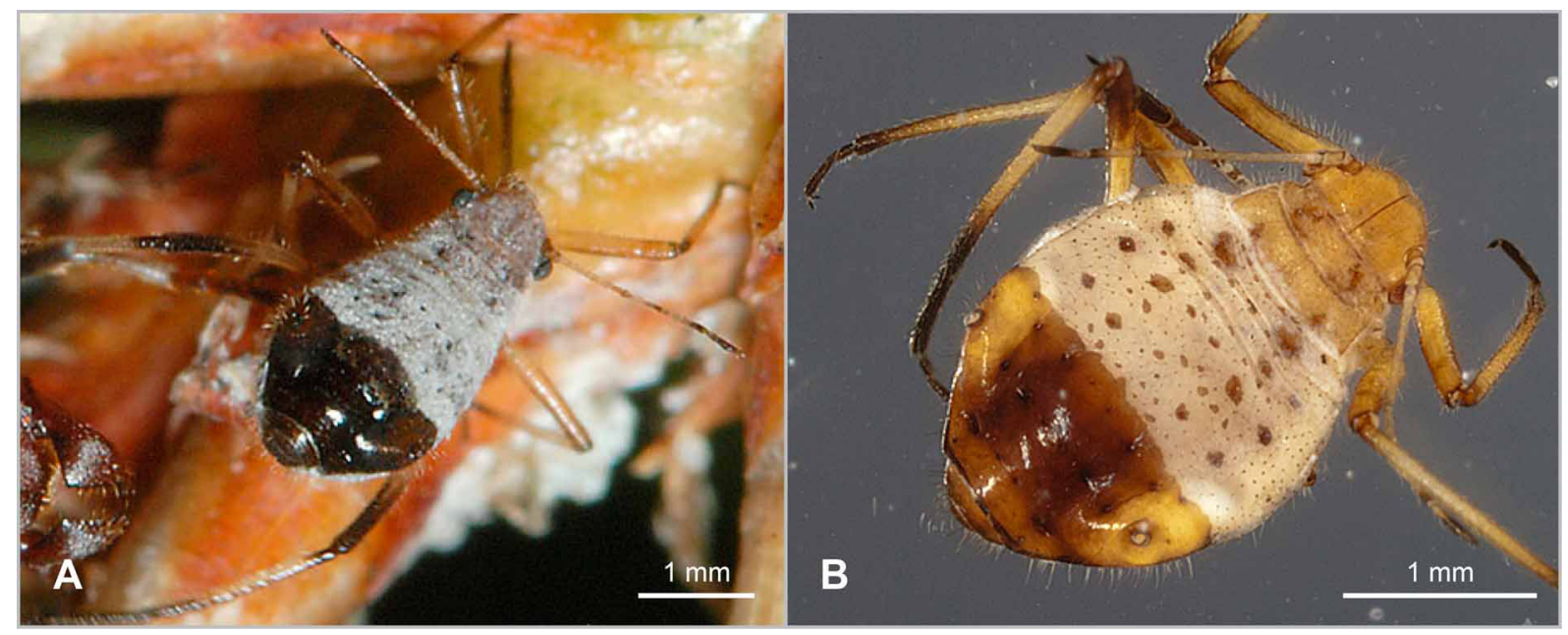

Fig. 98. Cinara (Cinara) brauni Börner, 1940. Apterae. A. In vivo on Pinus nigra. B. In alcohol. From Dransfield \& Brightwell 2015 (downloaded on 20 Mar. 2016). 


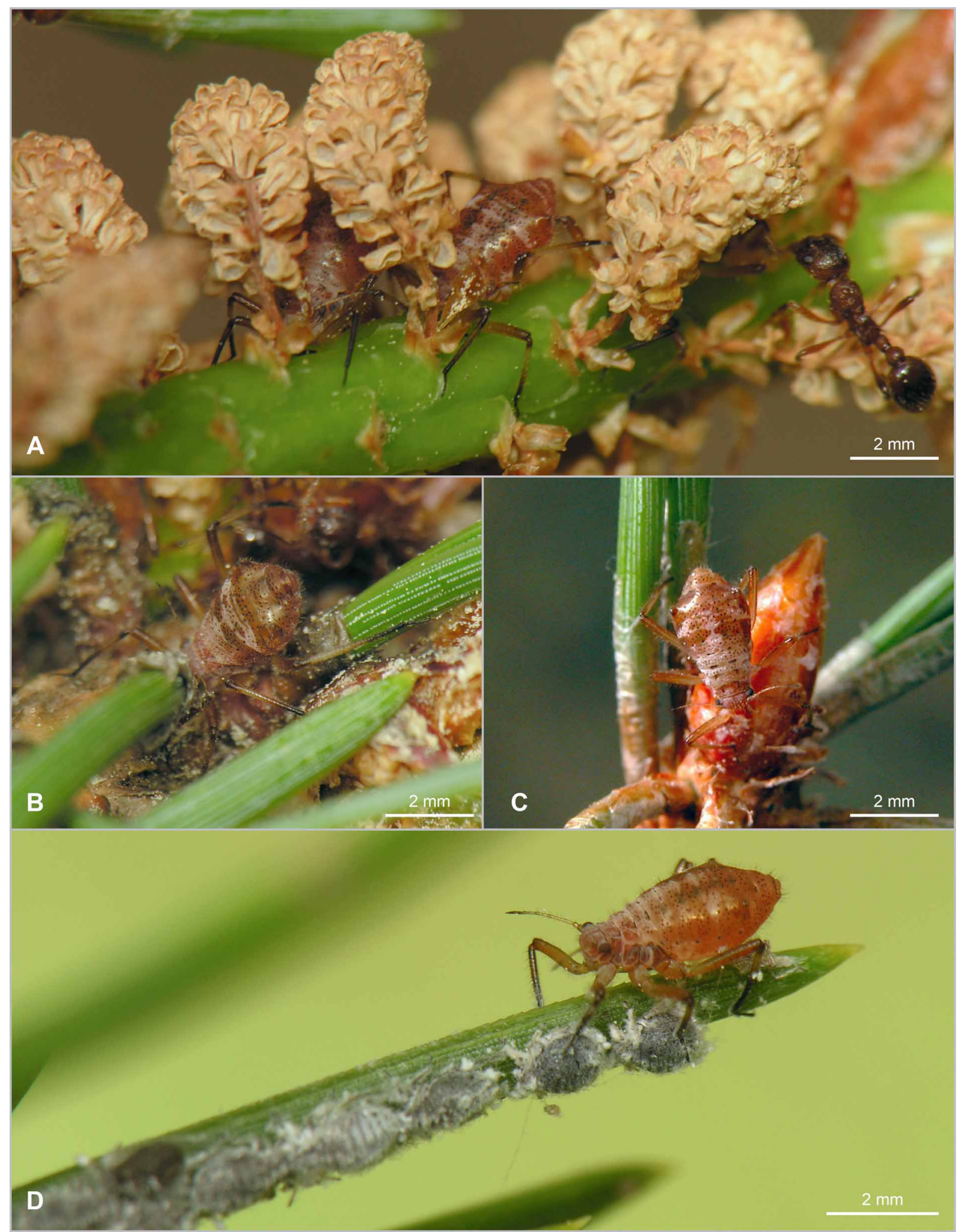

Fig. 99. Cinara (Cinara) pilosa (Zetterstedt, 1840). Apterae on old Pinus sylvestris. A-B. Small colony between and below male flowers, attended by Myrmica rubra (Linnaeus, 1758). C. Aptera on terminal bud. D. Aptera on needle in colony of Schizolachnus pineti. 


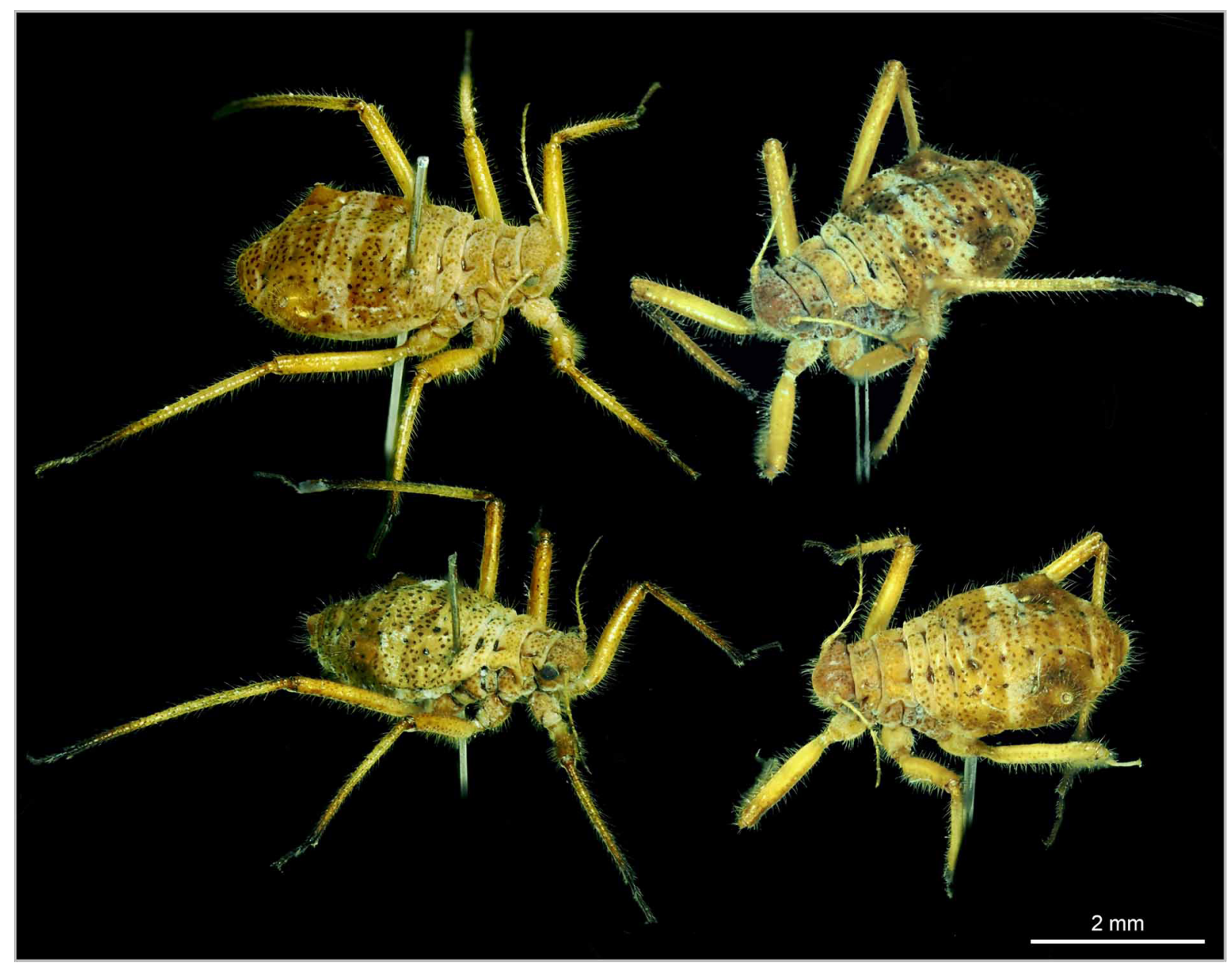

Fig. 100. Cinara (Cinara) pilosa (Zetterstedt, 1840). Apterae (freeze-dried specimens). 


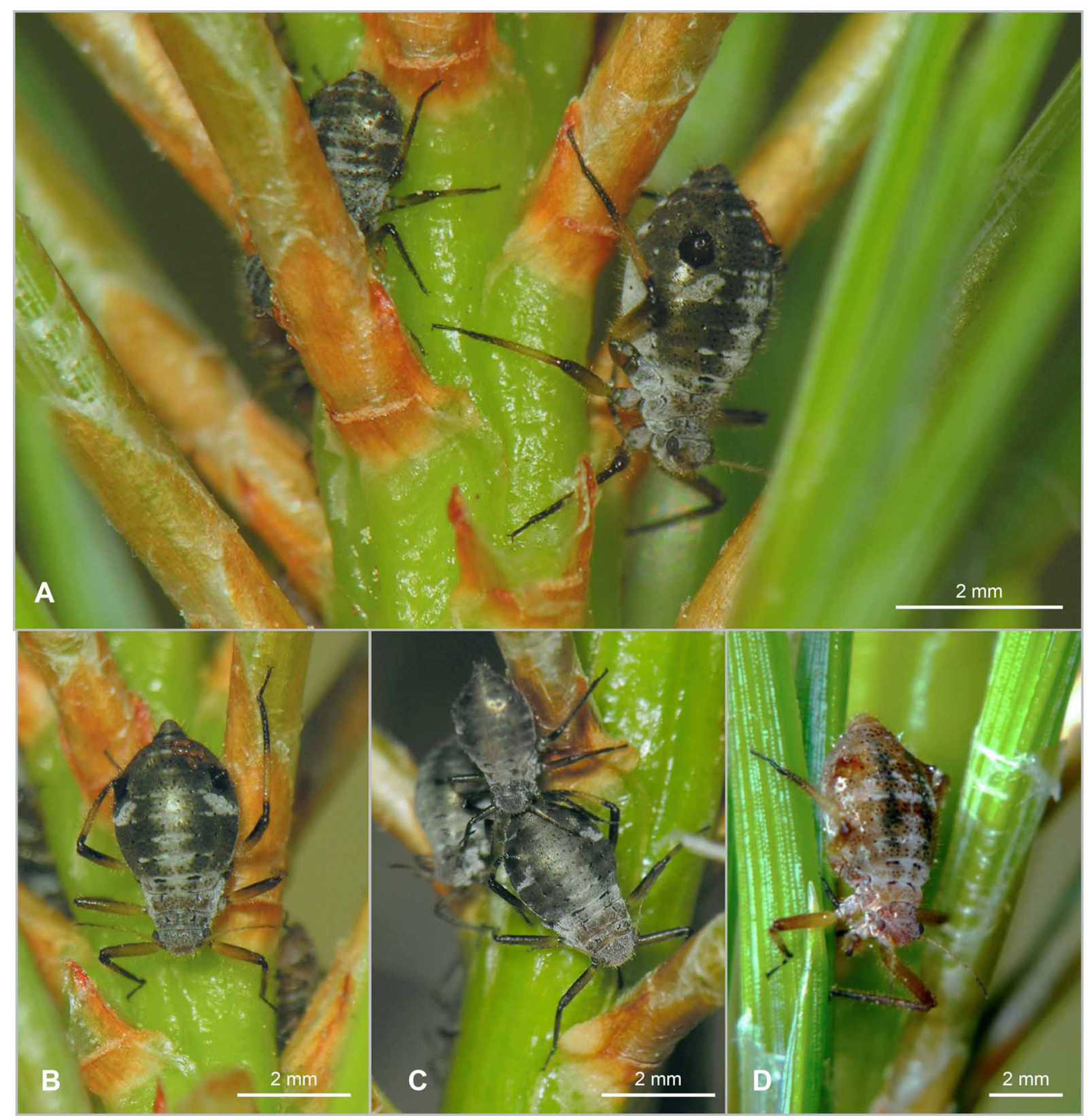

Fig. 101. Cinara (Cinara) pinea (Mordvilko, 1895). Apterae and juveniles on young shoots of Pinus sylvestris. 


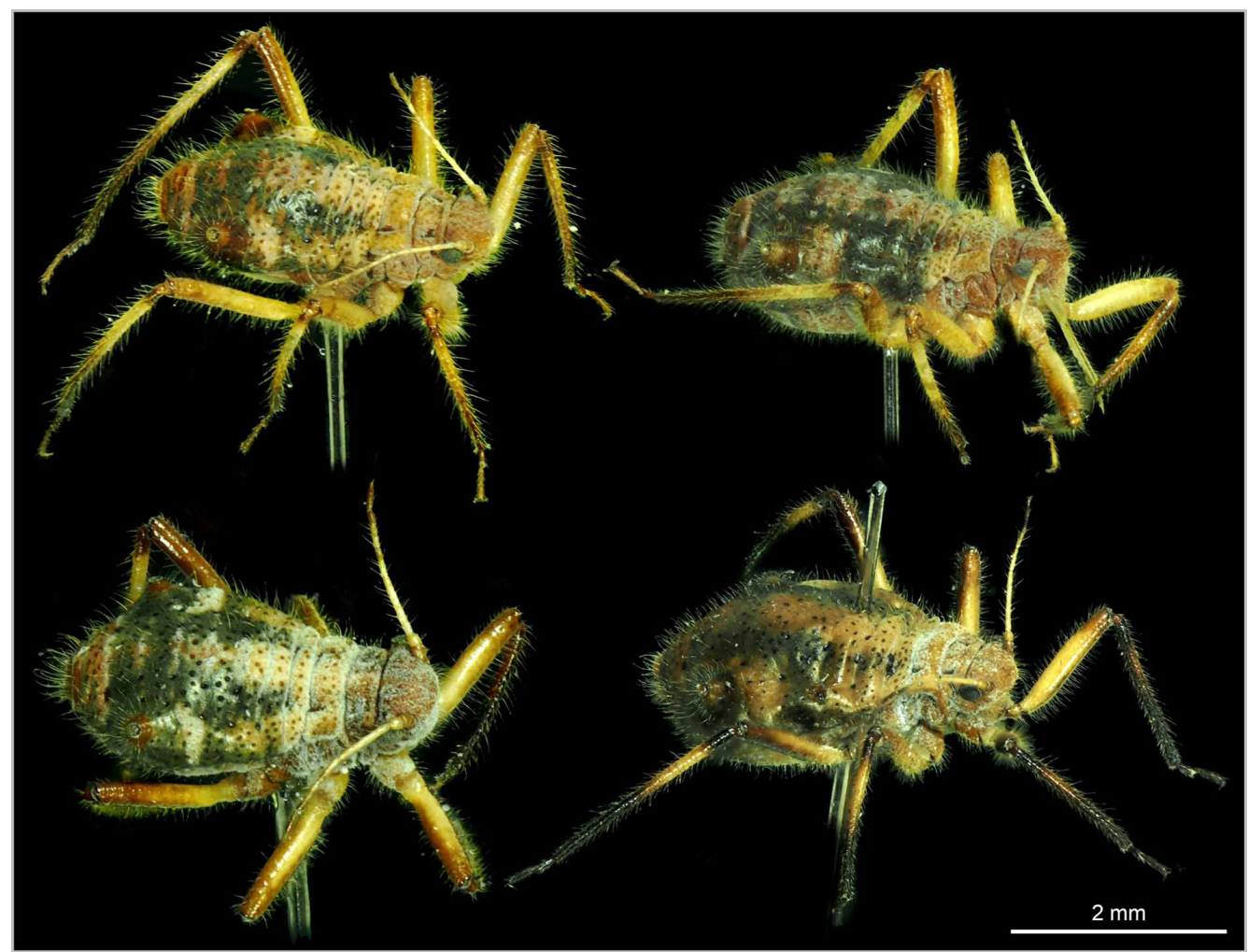

Fig. 102. Cinara (Cinara) pinea (Mordvilko, 1895). Apterae (freeze-dried specimens).

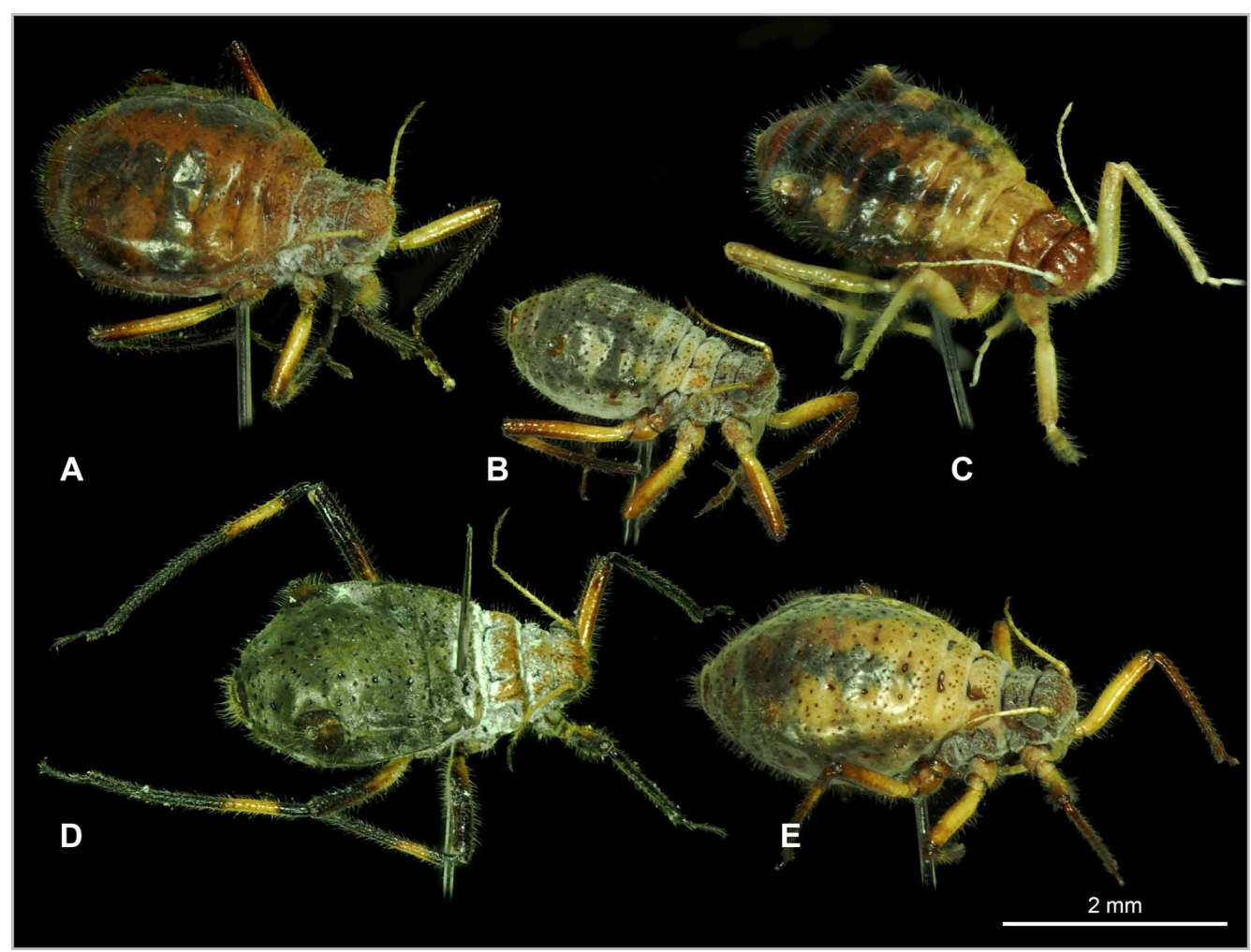

Fig. 103. Cinara (Cinara) pinea (Mordvilko, 1895). Freeze-dried specimens. A, C-D. Adult apterae. Teneral, appendages and hair-base sclerites still pale on C. B, E. Juvenile apterae. 


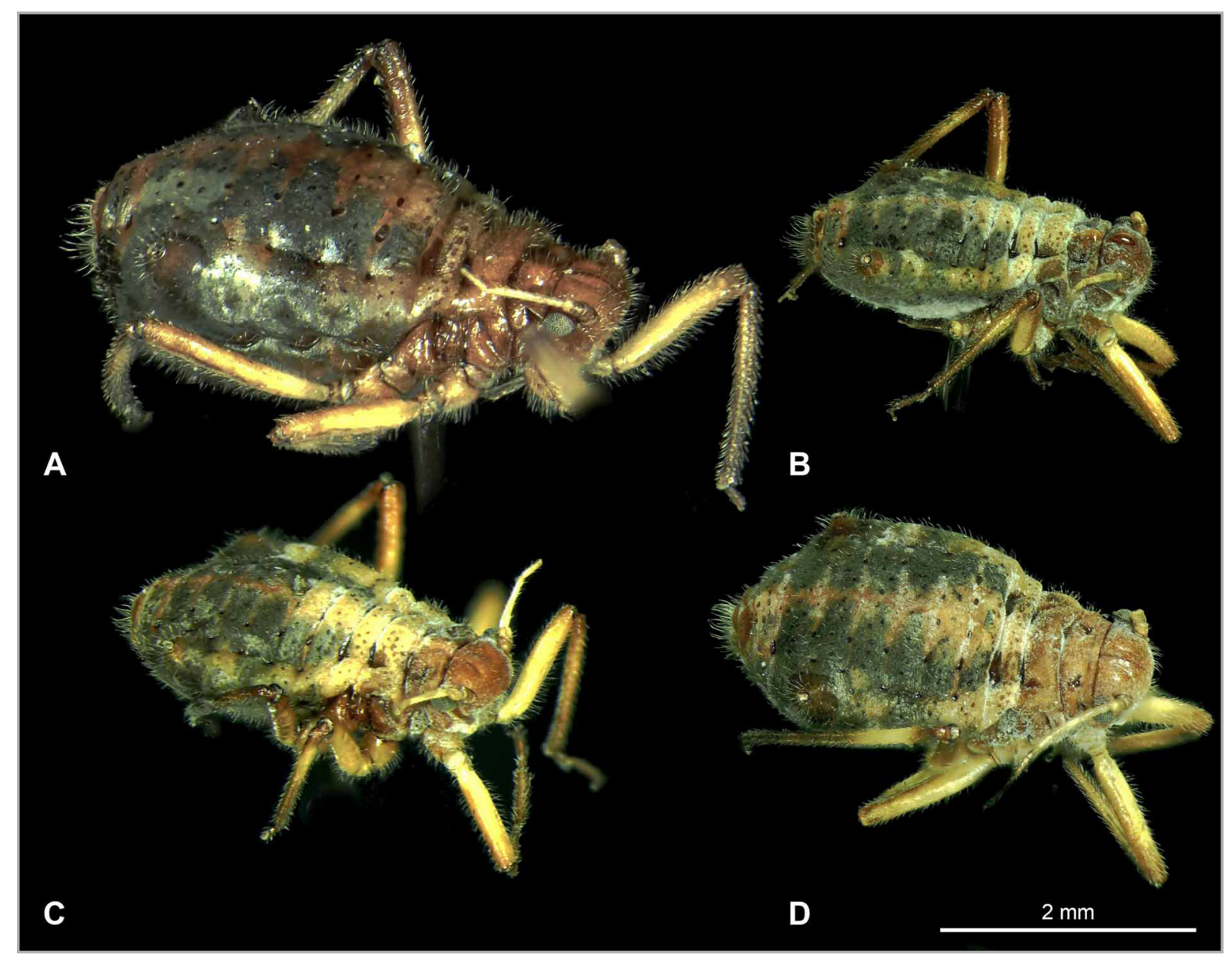

Fig. 104. Cinara (Cinara) piniphila (Ratzeburg, 1844). Freeze-dried specimens. 


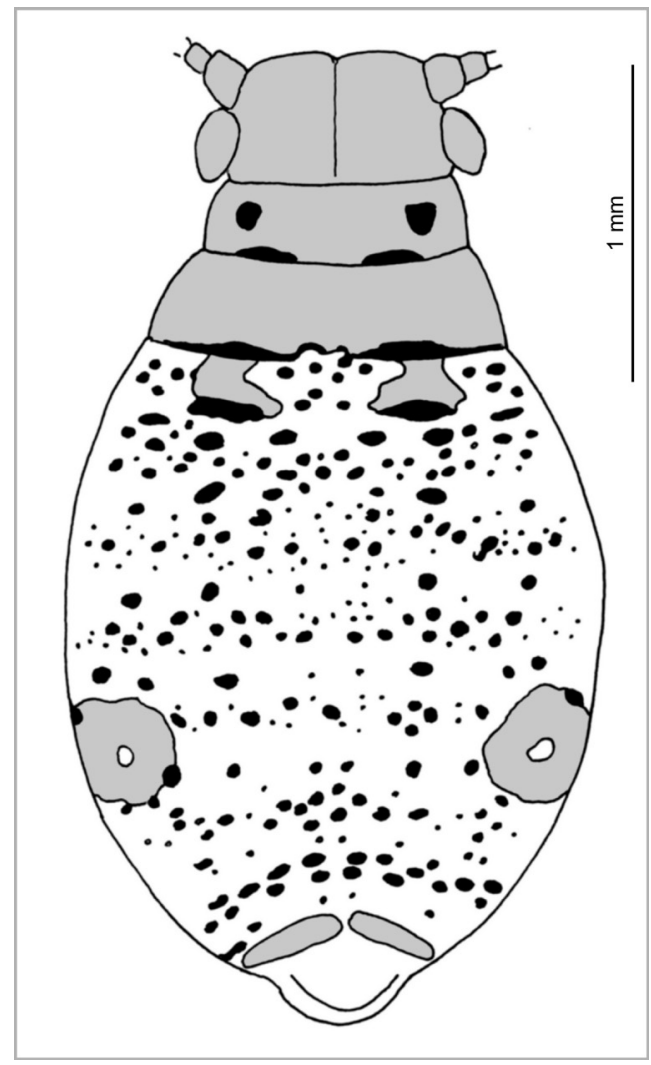

Fig. 105. Cinara (Cinara) piniphila (Ratzeburg, 1844). Aptera (from Pintera 1966, redrawn).

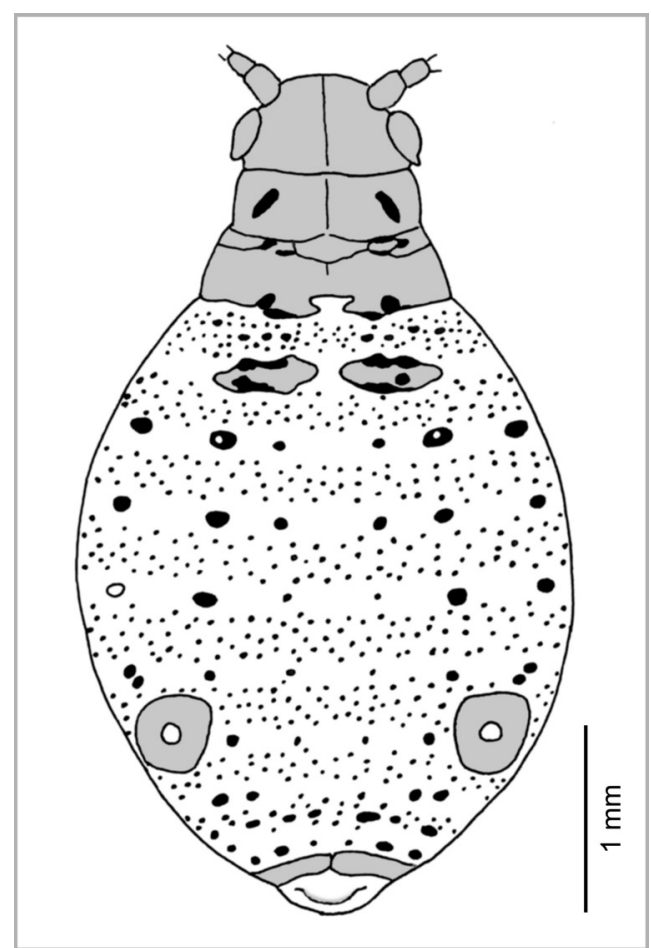

Fig. 106. Cinara (Cinara) schimitscheki Börner, 1940. Aptera (after Pintera 1966, redrawn). 


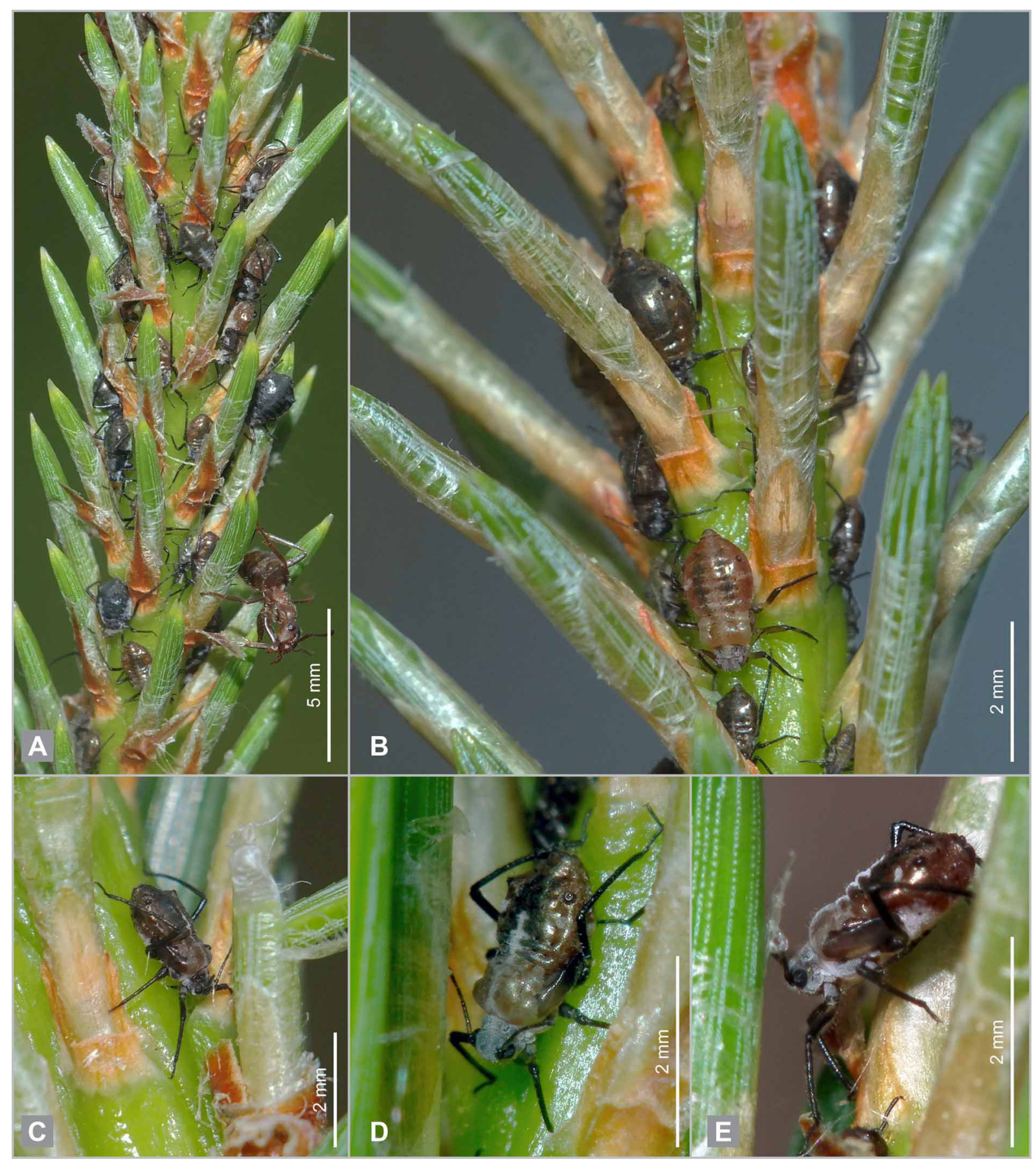

Fig. 107. Cinara (Cinara) hyperophila (Koch, 1855). Colony on young shoot of Pinus sylvestris, attended by Lasius niger (Linnaeus, 1758). A-B. Adult apterae and juvenile alatae. C-D. Juvenile alatae. 

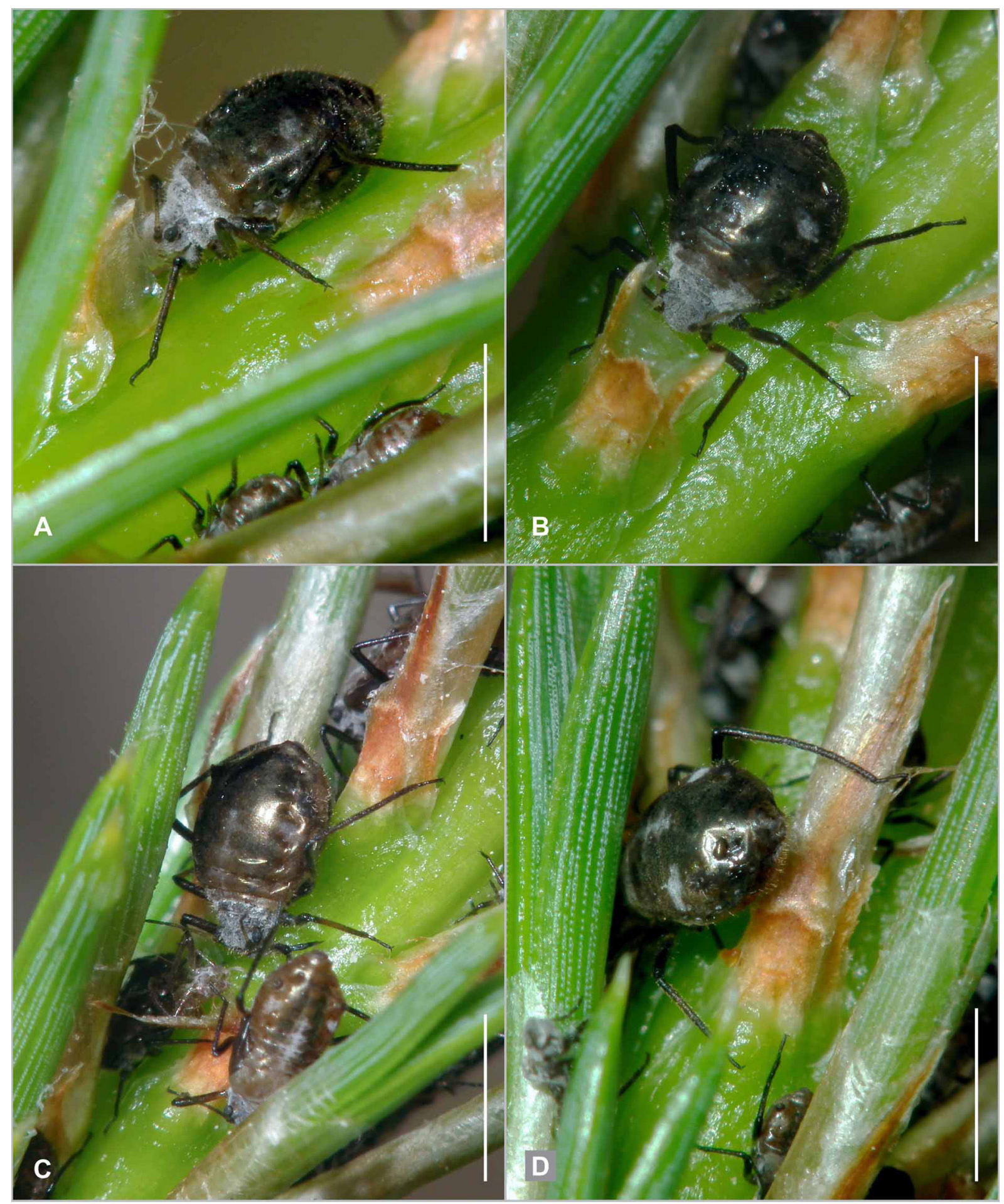

Fig. 108. Cinara (Cinara) hyperophila (Koch, 1855). Apterae and juvenile alatae on young shoot of Pinus sylvestris. Scale bars: $2 \mathrm{~mm}$. 


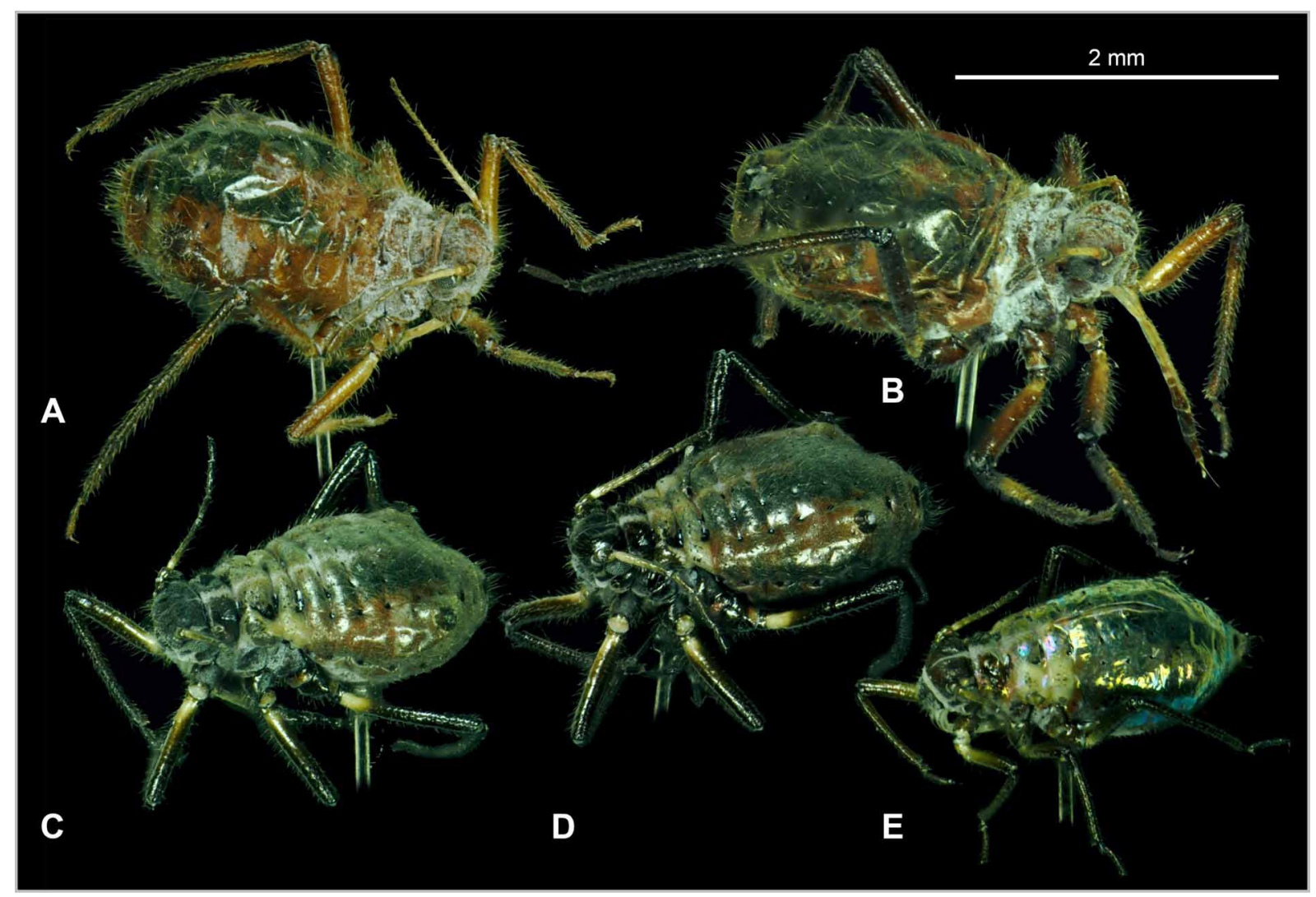

Fig. 109. Cinara (Cinara) hyperophila (Koch, 1855). Freeze-dried material. A-B. Adult apterae C-E. Juvenile apterae. 


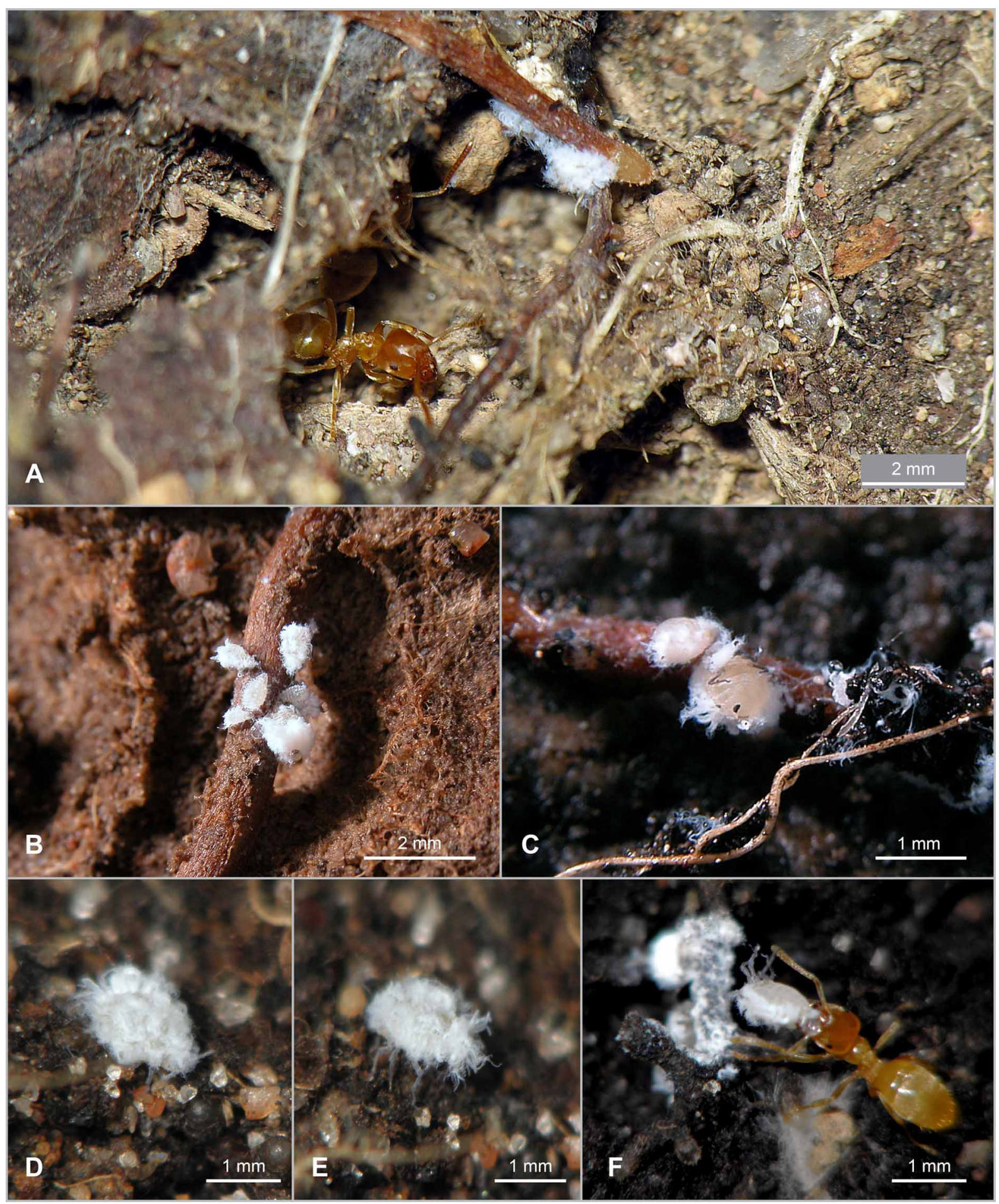

Fig. 110. Prociphilus (Stagona) pini (Burmeister, 1835). Apterae and juveniles on mycorrhizal roots of Pinus sylvestris in galleries of Lasius flavus (Fabricius, 1782). 


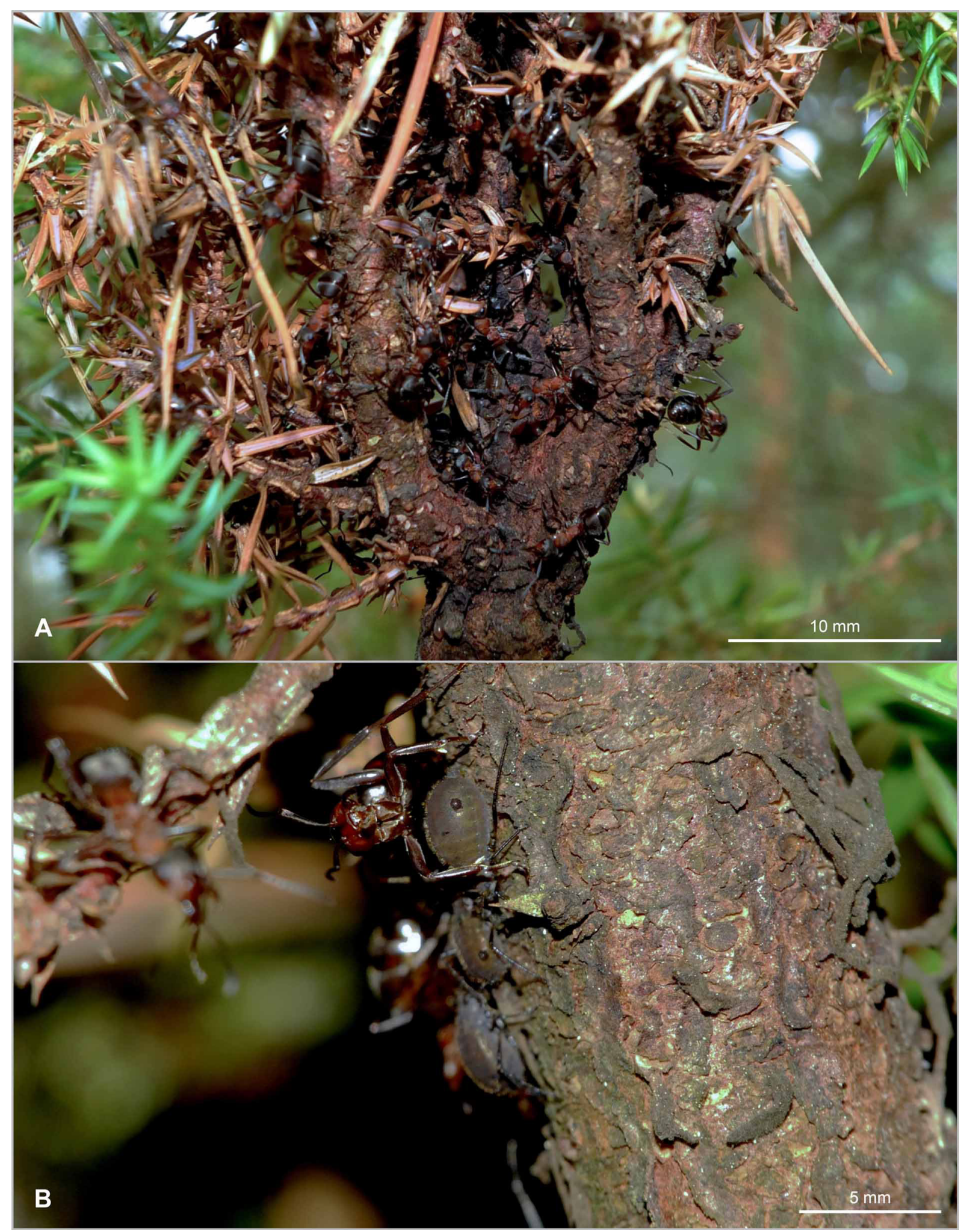

Fig. 111. Cinara (Cupressobium) smolandiae Danielsson \& Carter, 1993. Colony in Gymnosporangiuminduced wound on Juniperus communis, attended by Formica polyctena Förster, 1850. 


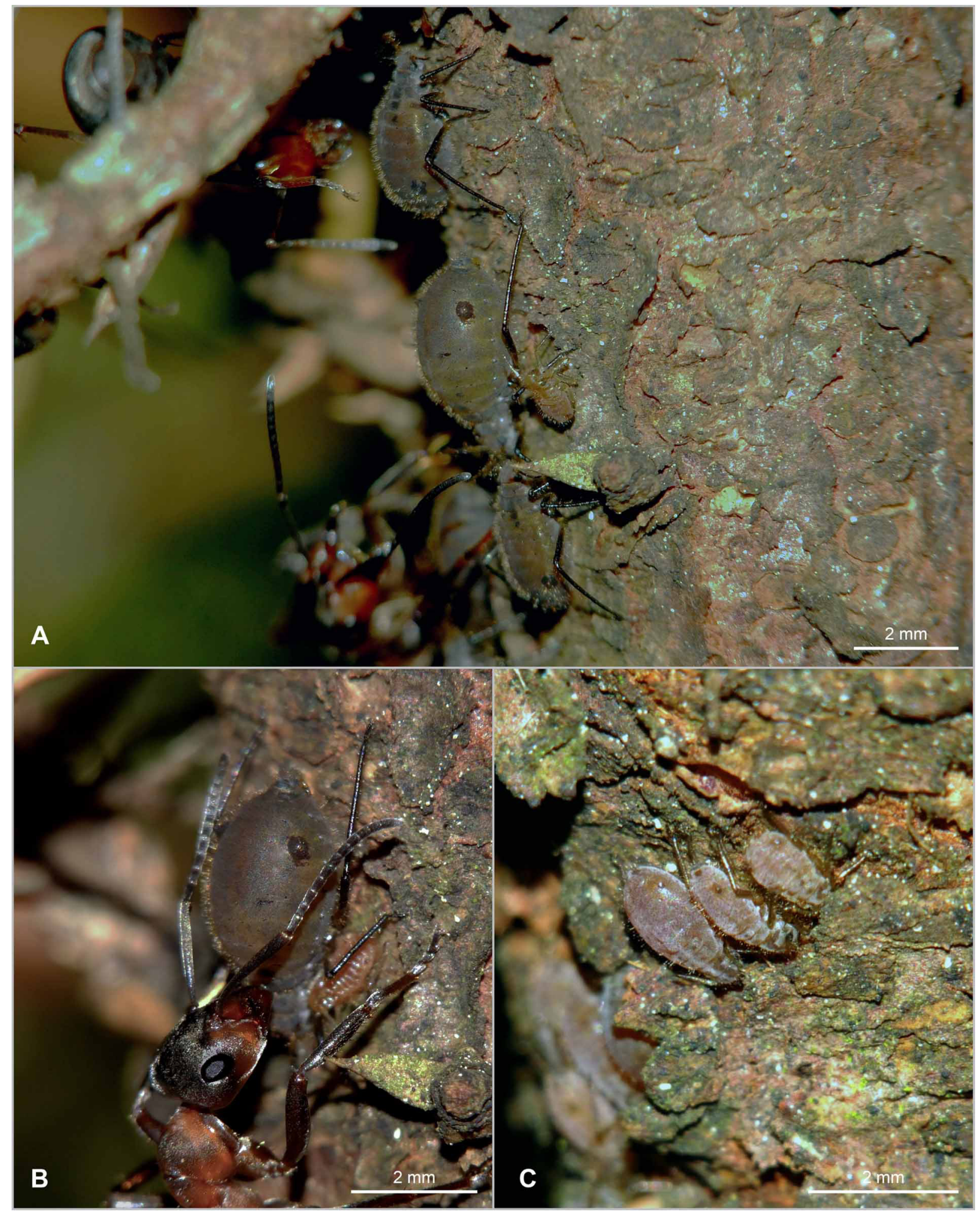

Fig. 112. Cinara (Cupressobium) smolandiae Danielsson \& Carter, 1993. Colony in Gymnosporangiuminduced wound on Juniperus communis, attended by Formica polyctena Förster, 1850. 


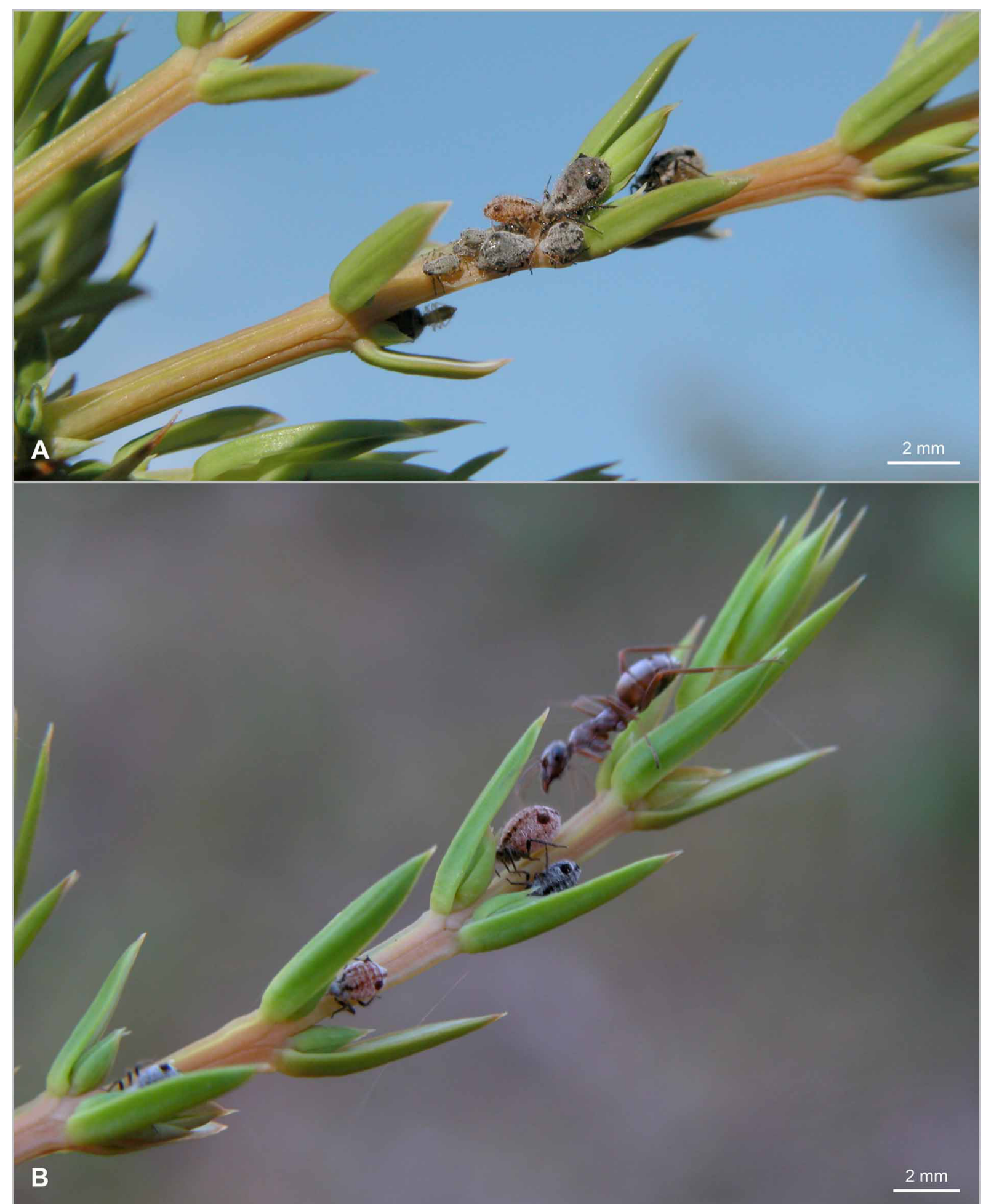

Fig. 113. Cinara (Cupressobium) juniperi (De Geer, 1773). Colonies on young shoots of Juniperus communis, in B attended by Formica cinerea Mayr, 1853. 


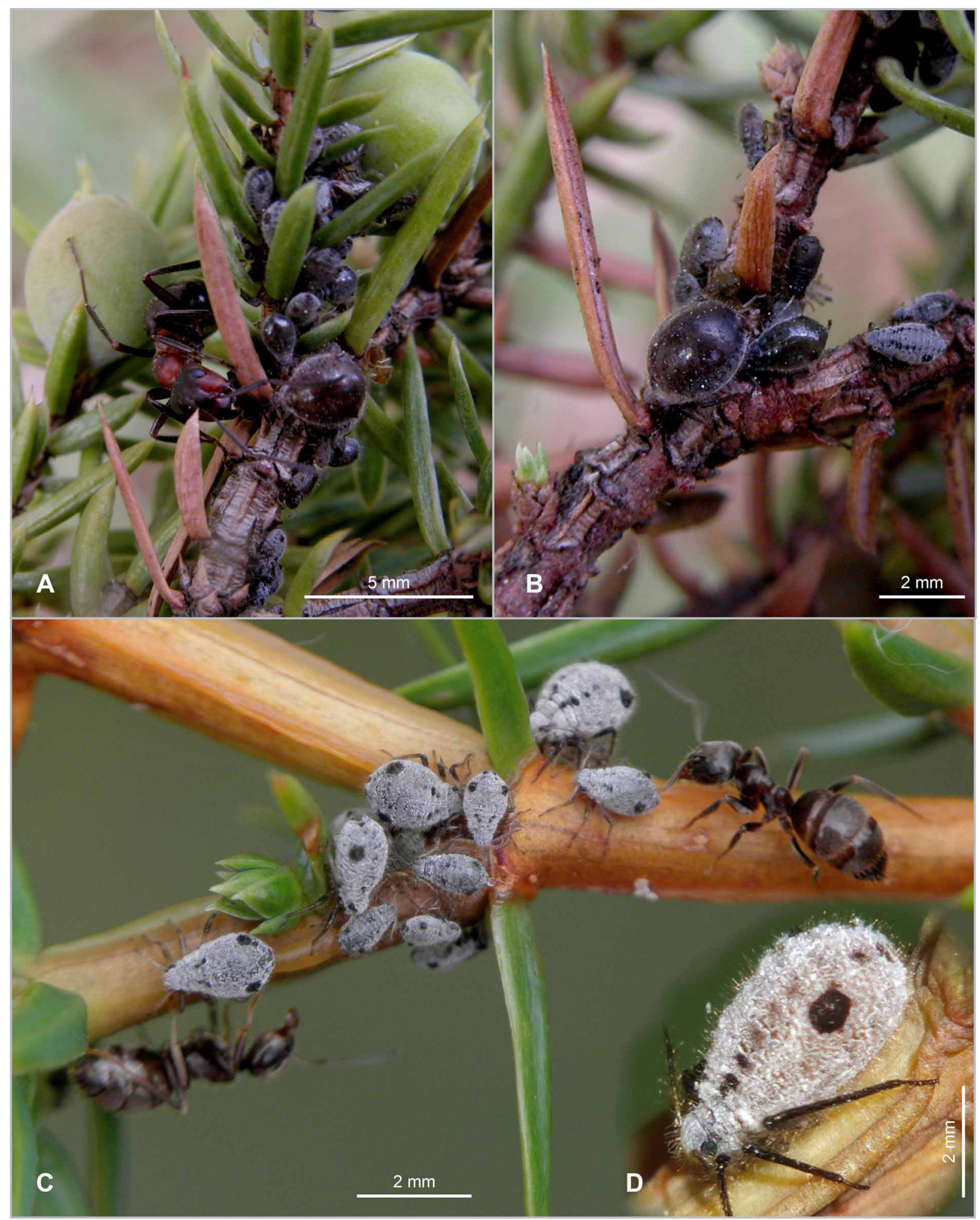

Fig. 114. Cinara (Cupressobium) juniperi (De Geer, 1773) on Juniperus communis. A-B. Fundatrix with offspring on previous year's twig, attended by Formica rufa Linnaeus, 1761. C-D. Colony on current year's growth, attended by Lasius niger (Linnaeus, 1758). 

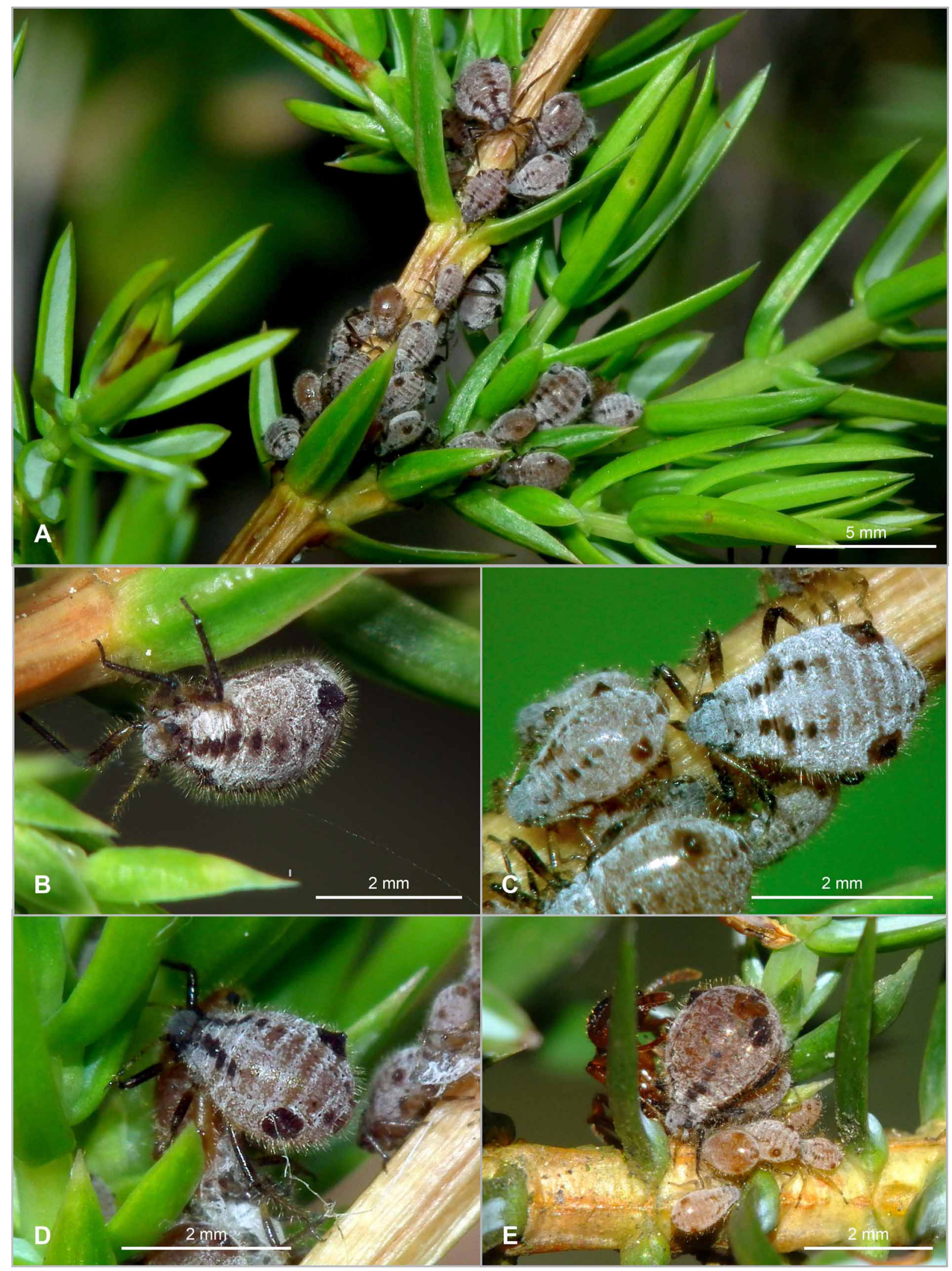

Fig. 115. Cinara (Cupressobium) juniperi (De Geer, 1773). Colonies on current and previous year's twigs of Juniperus communis, in F attended by Myrmica rubra (Linnaeus, 1758). 


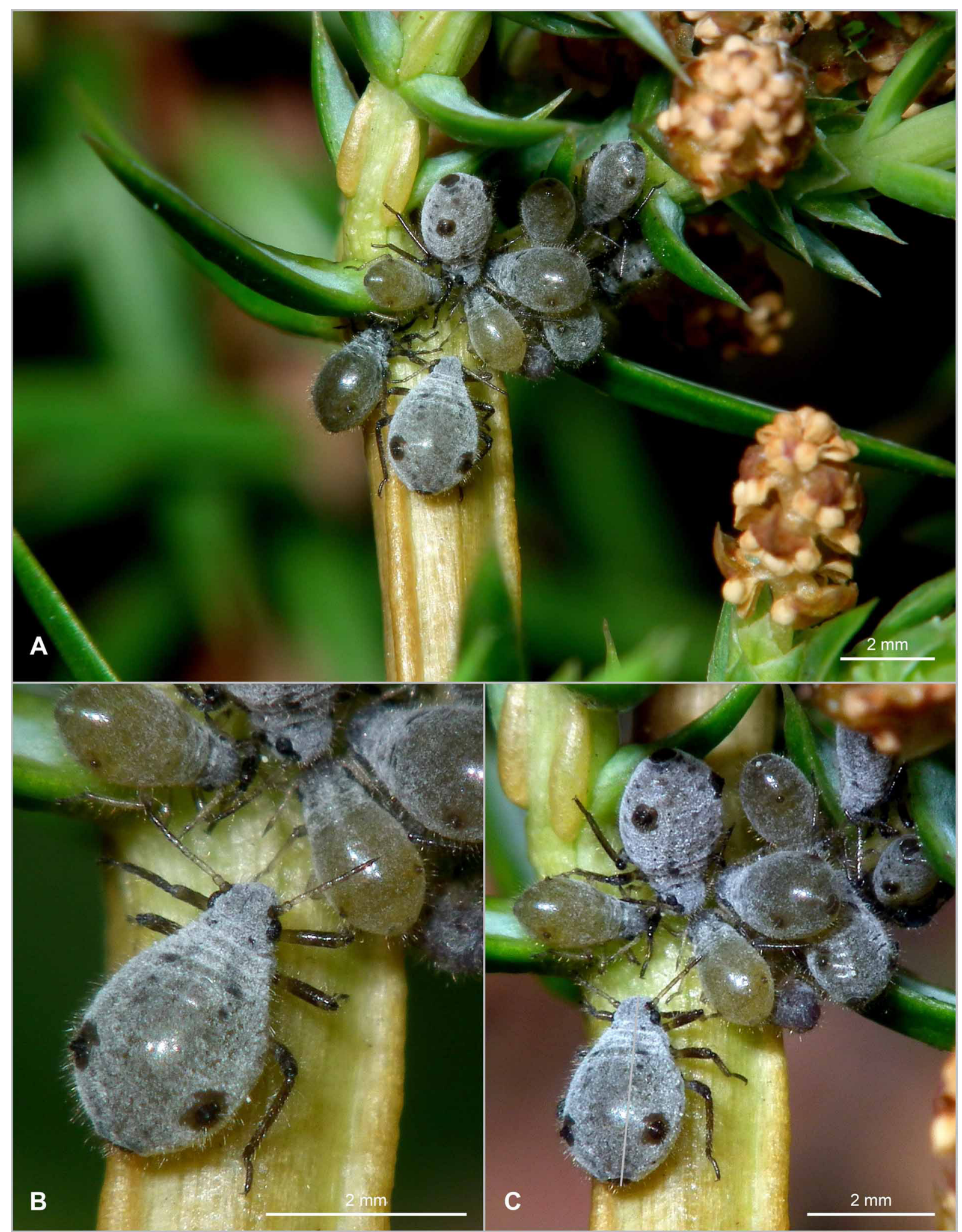

Fig. 116. Cinara (Cupressobium) mordvilkoi (Pasek, 1954). Colonies on current year's twigs of Juniperus communis. 


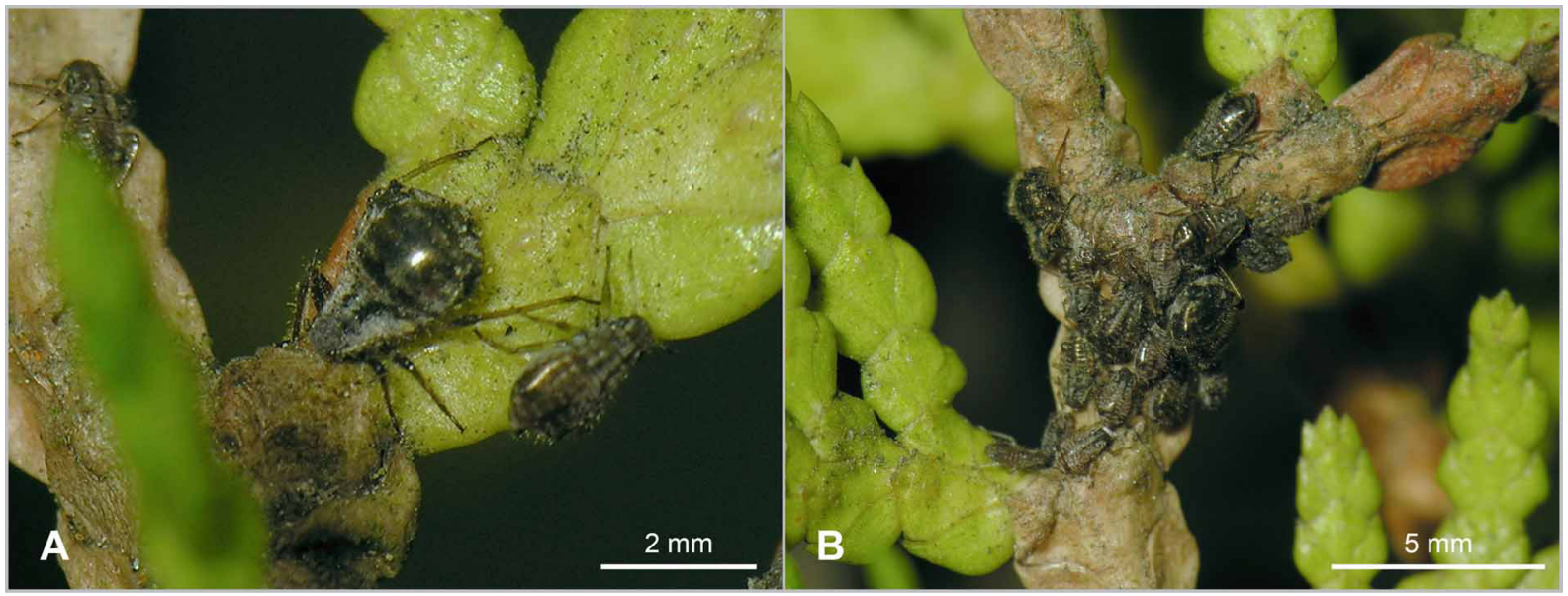

Fig. 117. Cinara (Cupressobium) cupressi (Buckton, 1881). Small colony on last year's growth of Thuja occidentalis, attended by Lasius niger (Linnaeus, 1758).

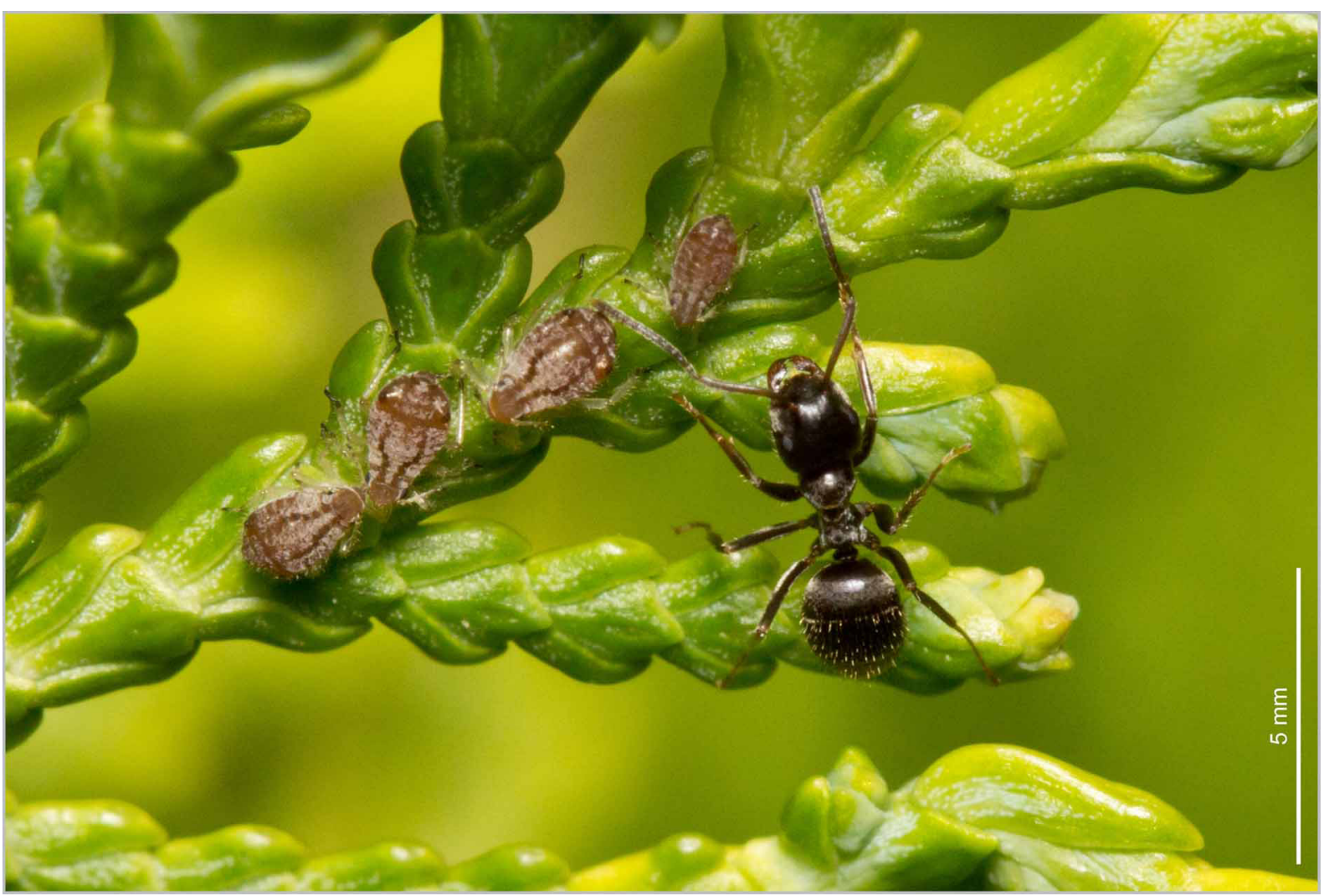

Fig. 118. Cinara (Cupressobium) tujafilina (Del Guercio, 1909) on young growth of Thuja occidentalis, attended by Lasius niger (Linnaeus, 1758). Photo Carlos Delgado (CCBYSA3.0, http://creativecommons. org/licenses/by-sa/3.0, downloaded 12 Nov. 2015 via https://commons.wikimedia.org/wiki/File:Lasius niger_y_Cinara_tujafilina_en_Thuja_orientalis.jpg\#filelinks). 


\section{Appendix 1}

\section{Summary of the host relationships of conifer-feeding aphids of northern Europe}

Dioecious species from primary host are denoted with ${ }^{\mathrm{I}}$, from secondary host with ${ }^{\mathrm{II}}$. ${ }^{*}=$ my own records from Finland. $\mathrm{N}^{*}$ and $\mathrm{S}^{*}=$ my own records from Norway and Sweden respectively.

PINOPHYTA Gorozh.

PINALES Gorozh.

Pinaceae Spreng.

Abies Mill.

Adelges laricis ${ }^{\mathrm{II}}$

Adelges nordmannianae II

Adelges pectinatae ${ }^{\mathrm{II}}$

Adelges piceae

Cinara confinis

Cinara curvipes

Cinara pectinatae

Elatobium abietinum

Prociphilus bumeliae ${ }^{\text {II }}$

Prociphilus fraxini ${ }^{\text {II }}$

Abies alba Mill.

Adelges merkeri ${ }^{\text {II }}$

Adelges nordmannianae $\mathrm{S}^{*} \mathrm{II}$

Adelges pectinatae* II

Adelges piceae

Cinara confinis

Cinara pectinatae $\mathrm{S}^{*}$

Mindarus abietinus

Prociphilus bumeliae II

Prociphilus fraxini II

Prociphilus xylostei? ${ }^{\text {II }}$

Abies amabilis (Dougl. ex Loud.) Dougl. ex Forbes

Adelges pectinatae* II

Adelges piceae

Abies balsamea (L.) Mill.

Adelges nordmannianae II

Adelges pectinatae* II

Adelges piceae

Cinara confinis

Cinara curvipes

Cinara pectinatae

Mindarus abietinus

Prociphilus fraxini ${ }^{\text {II }}$

Prociphilus xylostei? II

Abies cephalonica Loudon

Adelges nordmannianae II

Adelges piceae

Cinara confinis

Elatobium abietinum

Mindarus abietinus 
Abies concolor (Gordon \& Glend.) Lindl. ex Hildebr.

Adelges nordmannianae II

Adelges pectinatae* II

Adelges piceae

Cinara confinis

Cinara curvipes

Mindarus abietinus

Abies forrestii Coltm.-Rog.

Adelges piceae

Abies fraseri (Pursh) Poir.

Adelges pectinatae* II

Abies grandis (Douglas ex D. Don) Lindl.

Adelges nordmannianae II

Adelges pectinatae* II

Adelges piceae

Cinara confinis

Cinara pectinatae

Mindarus abietinus

Abies holophylla Maxim.

Cinara confinis*

Abies homolepis Siebold \& Zucc.

Elatobium abietinum

Mindarus abietinus

Abies koreana E. H. Wilson

Adelges pectinatae* II

Adelges piceae

Cinara curvipes

Abies lasiocarpa (Hook.) Nutt.

Adelges pectinatae* II

Adelges piceae

Cinara confinis

Cinara curvipes

Elatobium abietinum

Mindarus abietinus

Abies nordmanniana (Steven) Spach

Adelges merkeri? ${ }^{\mathrm{II}}$

Adelges nordmannianae ${ }^{\text {II }}$

Adelges pectinatae II

Adelges piceae

Cinara confinis

Cinara pectinatae

Elatobium abietinum

Mindarus abietinus

Pineus strobi? II

Prociphilus fraxini II

Abies pindrow (Royle ex D. Don) Royle

Cinara confinis

Mindarus abietinus

Abies procera Rehder

Adelges nordmannianae II 
Adelges pectinatae II

Adelges piceae

Cinara confinis

Mindarus abietinus

Abies sibirica Ledeb.

Adelges pectinatae* II

Adelges nordmannianae II

Adelges piceae

Cinara confinis*

Cinara pectinatae

Mindarus abietinus*

Prociphilus fraxini ${ }^{\text {II }}$

Abies veitchii Lindl.

Adelges pectinatae II

Adelges nordmannianae II

Cinara pectinatae

Mindarus abietinus

Prociphilus bumeliae II

Larix Mill.

Adelges laricis II

Adelges viridis ${ }^{\mathrm{II}}$

Cinara cuneomaculata

Cinara kochiana

Cinara laricis

Larix decidua Mill.

Adelges laricis* II

Adelges viridanus

Adelges viridis* II

Aphis fabae II

Cinara cuneomaculata*

Cinara kochiana

Cinara laricis*

Larix decidua $\times$ kaempferi

Adelges laricis ${ }^{\text {II }}$

Adelges viridis ${ }^{\mathrm{II}}$

Adelges viridanus

Cinara laricis

Larix gmelinii (Rupr.) Kuzen.

Adelges laricis ${ }^{\mathrm{II}}$

Adelges viridanus

Cinara cuneomaculata

Cinara confinis?

Cinara kochiana

Cinara laricis

Larix kaempferi (Lamb.) Carrière

Adelges laricis* II

Adelges viridanus

Adelges viridis ${ }^{\mathrm{II}}$

Aphis fabae II

Cinara cuneomaculata* 
Cinara kochiana

Cinara laricis

Larix laricina (Du Roi) K. Koch

Adelges laricis* II

Cinara cuneomaculata

Larix lyallii Parl.

Adelges laricis* II

Larix sibirica Ledeb.

Adelges laricis* II

Adelges tardoides ${ }^{\text {II }}$

Adelges viridanus

Adelges viridis*

Adelges viridulus II

Cinara cuneomaculata*

Cinara kochiana

Cinara laricis*

Picea A. Dietr.

Picea abies (L.) H. Karst.

Adelges abietis* $\mathrm{S} *$

Adelges lapponicus*

Adelges laricis* $\mathrm{S} *$

Adelges nordmannianae

Adelges pectinatae

Adelges tardoides

Adelges tardus* $\mathrm{S}^{*}$

Adelges viridis*

Aulacorthum solani

Cinara costata*

Cinara piceae*

Cinara piceicola*

Cinara pilicornis*

Cinara pruinosa*

Elatobium abietinum*

Pachypappa populi* II

Pachypappa tremulae* II

Pachypappa vesicalis II

Pachypappella lactea* II

Pineus cembrae

Pineus pineoides*

Prociphilus xylostei* II

Picea engelmannii (W. Parry ex Engelm.) Engelm.

Adelges abietis

Adelges cooleyi ${ }^{\mathrm{I}}$

Adelges viridis ${ }^{\mathrm{I}}$

Cinara costata

Cinara pilicornis

Elatobium abietinum

Mindarus obliquus

Prociphilus xylostei ${ }^{\text {II }}$ 
ALBRECHT A.C., Identification guide to Nordic aphids on conifers

Picea glauca (Moench) Voss Adelges abietis

Adelges cooleyi ${ }^{\mathrm{I}}$

Adelges laricis ${ }^{\mathrm{I}}$

Adelges lapponicus*

Adelges pectinatae ${ }^{\mathrm{I}}$

Adelges tardus

Adelges viridis* I

Cinara costata*

Cinara piceae

Cinara piceicola

Cinara pilicornis*

Cinara pruinosa

Elatobium abietinum

Mindarus obliquus*

Pachypappa vesicalis II

Prociphilus xylostei II

Picea glehnii (Fr. Schmidt) Masters

Adelges pectinatae ${ }^{\mathrm{I}}$

Cinara costata

Cinara piceae

Cinara pilicornis

Elatobium abietinum

Pineus cembrae ${ }^{\mathrm{I}}$

Picea jezoensis (Sieb.\& Zucc.) Carriere

Adelges abietis

Adelges cooleyi ${ }^{\mathrm{I}}$

Adelges lapponicus

Adelges pectinatae ${ }^{\mathrm{I}}$

Cinara costata

Cinara piceae

Cinara piceicola

Cinara pilicornis

Elatobium abietinum

Pineus cembrae ${ }^{\mathrm{I}}$

Pineus orientalis ${ }^{\mathrm{I}}$

Picea koraiensis Nakai

Adelges laricis ${ }^{\mathrm{I}}$

Adelges pectinatae ${ }^{\mathrm{I}}$

Adelges viridis ${ }^{\mathrm{I}}$

Cinara piceae

Cinara pilicornis

Cinara pruinosa

Pineus cembrae ${ }^{\mathrm{I}}$

Picea mariana (Mill.) Britton, Sterns \& Poggenb.

Adelges cooleyi ${ }^{\mathrm{I}}$

Adelges lapponicus

Adelges laricis ${ }^{\mathrm{I}}$

Cinara costata

Elatobium abietinum 
Mindarus obliquus

(Pineus strobi, see text) ${ }^{\mathrm{I}}$

Picea omorika (Pancic) Purk.

Adelges nordmannianae ${ }^{\mathrm{I}}$

Adelges viridis ${ }^{\mathrm{I}}$

Cinara costata*

Cinara picea**

Cinara pilicornis*

Elatobium abietinum

Mindarus obliquus

Pineus pineoides

Picea orientalis (L.) Link

Adelges abietis

Adelges laricis ${ }^{\mathrm{I}}$

Adelges merkeri ${ }^{\mathrm{I}}$

Adelges nordmannianae ${ }^{\mathrm{I}}$

Adelges viridis ${ }^{\mathrm{I}}$

Cinara costata

Cinara piceae

Cinara piceicola

Cinara pilicornis

Cinara pruinosa

Elatobium abietinum

Pachypappa tremulae II

Pineus cembrae ${ }^{\mathrm{I}}$

Pineus orientalis* $\mathrm{I}$

Picea pungens Engelm.

Adelges abietis

Adelges cooleyi ${ }^{\mathrm{I}}$

Adelges lapponicus

Adelges laricis ${ }^{\mathrm{I}}$

Adelges tardus

Adelges viridis ${ }^{\mathrm{I}}$

Cinara costata*

Cinara piceae

Cinara piceicola

Cinara pilicornis

Cinara pruinosa

Elatobium abietinum*

Pineus orientalis ${ }^{\mathrm{I}}$

Picea sitchensis (Bong.) Carrière

Adelges abietis*

Adelges cooleyi (usually) ${ }^{\mathrm{I}}$

Adelges lapponicus

Adelges laricis ${ }^{\mathrm{I}}$

Adelges pectinatae ${ }^{\mathrm{I}}$

Adelges tardus

Adelges viridis ${ }^{\mathrm{I}}$

Aphis fabae II

Aulacorthum solani 
ALBRECHT A.C., Identification guide to Nordic aphids on conifers

Cinara costata

Cinara piceae

Cinara piceicola

Cinara pilicornis

Cinara pruinosa

Elatobium abietinum

Mindarus obliquus

Neomyzus circumflexus

Pachypappa tremulae II

Pachypappa vesicalis ${ }^{\mathrm{II}}$

Pachypappella lactea II

Pineus similis

Prociphilus xylostei ${ }^{\text {II }}$

Picea tianschanica Lindl.

Cinara costata

Cinara piceae

Cinara pilicornis

Cinara pruinosa

Elatobium abietinum

Picea torano (Siebold ex K.Koch) Koehne (syn. polita)

Pineus orientalis ${ }^{\mathrm{I}}$

Pinus L.

Pinus banksiana Lamb.

Cinara pinea*

Cinara pini?

Eulachnus agilis*

Eulachnus alticola

Eulachnus rileyi

Pineus pini

Schizolachnus pineti*

Pinus cembra L.

Cinara cembrae

Cinara pini?

Eulachnus agilis

Eulachnus cembrae

Eulachnus rileyi

Pineus cembrae* II

Schizolachnus pineti

Pinus contorta Douglas ex Loudon

Aphis fabae II

Cinara pini?

Prociphilus pini ${ }^{\text {II }}$

Schizolachnus pineti

Pinus koraiensis Sieb. \& Zucc.

Cinara kochiana?

Pineus cembrae II

Pinus mugo Turra

Cinara hyperophila

Cinara neubergi

Cinara nuda 
Cinara pilosa*

Cinara pinea*

Cinara pini*

Cinara pinihabitans

Cinara schimitscheki

Eulachnus alticola

Eulachnus agilis*

Eulachnus brevipilosus

Eulachnus rileyi*

Pineus orientalis II

Pineus pini*

Schizolachnus obscurus

Schizolachnus pineti*

Pinus nigra J. F. Arnold

Cinara acutirostris

Cinara brauni

Cinara nuda

Cinara pilosa

Cinara pinea $\mathrm{S}^{*}$

Cinara pini?

Cinara schimitscheki

Eulachnus agilis

Eulachnus brevipilosus

Eulachnus nigricola

Eulachnus rileyi

Pineus pini

Schizolachnus obscurus

Schizolachnus pineti

Pinus peuce Griseb.

Eulachnus cembrae

Eulachnus rileyi

Pineus strobi ${ }^{\text {II }}$

Pinus pinaster Aiton

Cinara hyperophila

Cinara pini?

Eulachnus agilis

Eulachnus brevipilosus

Eulachnus rileyi

Pineus pini

Schizolachnus obscurus

Schizolachnus pineti

Pinus pinea L.

Cinara acutirostris

Cinara pini?

Cinara pinea

Cinara schimitscheki

Eulachnus agilis

Eulachnus rileyi

Pineus pini

Schizolachnus obscurus 
Schizolachnus pineti

Pinus ponderosa Douglas ex Lawson

Cinara pinea

Eulachnus alticola

Pineus pini

Schizolachnus pineti

Pinus pumila (Pall.) Regel

Pineus cembrae

Pinus rigida Mill.

Eulachnus agilis

Eulachnus alticola

Eulachnus rileyi

Pineus pini

Pinus sibirica Du Tour

Cinara brauni

Cinara pinea

Cinara pini?

Elatobium abietinum

Eulachnus agilis

Pineus cembrae ${ }^{\mathrm{II}}$

Pinus strobus L.

Cinara pini?

Eulachnus cembrae

Pineus strobi ${ }^{\text {II }}$

Pinus sylvestris L.

Cinara brauni

Cinara hyperophila*

Cinara nuda*

Cinara nigritergi* $\mathrm{S}^{*}$

Cinara pilosa* $\mathrm{N}^{*}$

Cinara pinea*

Cinara pini*

Cinara pinihabitans*

Cinara piniphila*

Eulachnus agilis*

Eulachnus alticola

Eulachnus brevipilosus*

Eulachnus nigricola

Eulachnus rileyi

Pineus orientalis II

Pineus pini*

Prociphilus pini* II

Schizolachnus obscurus

Schizolachnus pineti*

Pseudotsuga Carrière

Prociphilus pini ${ }^{\mathrm{II}}$

Pseudotsuga menziesii (Mirb.) Franco

Adelges cooleyi $\mathrm{S} *$ II

Elatobium abietinum

Mindarus abietinus 
Pachypappa vesicalis ${ }^{\mathrm{II}}$

Prociphilus xylostei ${ }^{\text {II }}$

Tsuga (Endl.) Carrière

Tsuga canadensis (L.) Carrière

Prociphilus pini ${ }^{\text {II }}$

Tsuga heterophylla (Raf.) Sarg.

Aphis fabae II

Cedrus Trew

Cinara pilicornis?

Cedrus deodara (Roxb.) G.Don

Cinara curvipes

CUPRESSALES Link

Cupressaceae Gray

Chamaecyparis Spach

Chamaecyparis lawsoniana (A. Murray bis) Parl.

Cinara cupressi

Cinara tujafilina

Cryptomeria D. Don

Cryptomeria japonica (L. f.) D. Don

Brachycaudus helichrysi? ${ }^{\mathrm{II}}$

Cinara fresai

Cupressus L.

Cinara cupressi

Cinara fresai

Juniperus L.

Cinara tujafilina

Cinara tujafilina

Juniperus chinensis L.

Cinara cupressi

Cinara juniperi

Cinara tujafilina

Juniperus communis L.

Cinara confinis?

Cinara cupressi

Cinara juniperi*

Cinara mordvilkoi*

Cinara smolandiae*

Cinara tujafilina

Gootiella tremulae II

Juniperus procumbens (Siebold ex Endl.) Miq.

Cinara tujafilina

Juniperus sabina L.

Cinara cupressi

Cinara juniperi

Gootiella tremulae II

Juniperus scopulorum Sarg.

Cinara cupressi

Juniperus squamata Buch.-Ham. ex Lamb.

Cinara juniperi

Juniperus virginiana $\mathrm{L}$. 
ALBRECHT A.C., Identification guide to Nordic aphids on conifers

Cinara cupressi

Cinara juniperi

Cinara tujafilina

Thuja L.

Cinara tujafilina

Thuja occidentalis L.

Cinara cupressi*

Cinara juniperi

Cinara tujafilina

Thuja plicata Donn ex D. Don

Cinara cupressi*

Myzus persicae? ${ }^{\mathrm{II}}$

Thujopsis Siebold \& Zucc.

Thujopsis dolabrata Siebold \& Zucc.

Cinara tujafilina 


\section{Appendix 2}

\section{Summary of the aphid-ant relationships of the conifer-feeding aphids of northern Europe}

Dioecious species from primary host are denoted with ${ }^{\mathrm{I}}$, from secondary host with ${ }^{\mathrm{II}} . *$ = my own records from Finland. S* = my own records from Sweden.

Formicidae Latreille, 1809

Myrmicinae Lepeletier, 1835

Myrmica Latreille, 1804

Myrmica rubra (Linnaeus, 1758)

Cinara juniperi*

Cinara piceicola*

Cinara pilicornis*

Cinara pilosa*

Cinara pinea*

Cinara pruinosa*

Myrmica ruginodis Nylander, 1846

Cinara pinea*

Cinara pruinosa*

Formicinae Lepeletier, 1836

Camponotus Mayr, 1861

Cinara smolandiae

Camponotus herculeanus (Linnaeus, 1758)

Cinara piceae*

Cinara pinea*

Cinara pini*

Camponotus ligniperda (Latreille, 1802)

Cinara pinea*

Lasius Fabricius, 1804

sg. Lasius Fabricius, 1804

Lasius (Lasius) alienus (Förster, 1850)

Cinara schimitscheki (alienus group?)

Lasius (Lasius) niger (Linnaeus, 1758)

Cinara cupressi*

Cinara hyperophila*

Cinara juniperi*

Cinara laricis*

Cinara nigritergi*

Cinara nuda*

Cinara picea**

Cinara piceicola*

Cinara pilicornis*

Cinara pinea*

Cinara pruinosa*

Cinara schimitscheki

Cinara tujafilina

Mindarus obliquus*

Lasius (Lasius) platythorax Seifert, 1991

Cinara hyperophila*

Cinara nuda* 
Cinara pinea*

sg. Cautolasius Wilson, 1955

Lasius (Cautolasius) flavus (Fabricius, 1782)

Prociphilus pini* II

sg. Dendrolasius Ruzsky, 1912

Lasius (Dendrolasius) fuliginosus (Latreille, 1798)

Cinara juniperi*

Cinara nigritergi $\mathrm{S}^{*}$

Cinara piceicola*

Formica Linnaeus, 1758

Cinara smolandiae

sg. Formica Linnaeus, 1758

Formica (Formica) pratensis Retzius, 1783

Cinara piceicola*

Cinara pini*

Formica (Formica) truncorum Fabricius, 1804

Cinara hyperophila*

Cinara nuda*

Cinara piceae*

Cinara piceicola*

Cinara pilicornis*

Cinara pinea*

Cinara pini*

Cinara pruinosa*

Formica (Formica) aquilonia Yarrow, 1955

Cinara nuda*

Cinara piceicola*

Cinara pilicornis*

Cinara pinea*

Cinara pini*

Cinara pruinosa*

Formica (Formica) lugubris Zetterstedt, 1838

Cinara kochiana

Cinara piceicola*

Cinara pilosa*

Cinara pruinosa*

Formica (Formica) polyctena Förster, 1850

Cinara hyperophila*

Cinara juniperi*

Cinara nuda*

Cinara pectinatae $\mathrm{S}^{*}$

Cinara piceae*

Cinara pinea*

Cinara pini*

Cinara pruinosa*

Cinara smolandiae*

Formica (Formica) rufa Linnaeus, 1761

Cinara acutirostris (F. rufa group?)

Cinara brauni (F. rufa group?)

Cinara cembrae (F. rufa group?) 
Cinara confinis*

Cinara cuneomaculata*

Cinara curvipes (F. rufa group?)

Cinara hyperophila*

Cinara juniperi*

Cinara laricis*

Cinara pinea*

Cinara pini*

sg. Coptoformica Müller, 1923

Formica (Coptoformica) pressilabris Nylander, 1846

Cinara pinea*

sg. Raptiformica Forel, 1913

Formica (Raptiformica) sanguinea Laverne, 1798

Cinara hyperophila*

Cinara piceicola*

Cinara pini*

Cinara schimitscheki

sg. Serviformica Forel, 1913

Formica (Serviformica) cinerea Mayr, 1853

Cinara hyperophila*

Cinara juniperi*

Cinara nuda*

Cinara pinea*

Cinara pini*

Cinara piniphila*

Formica (Serviformica) fusca Linnaeus, 1758

Cinara hyperophila*

Cinara juniperi*

Cinara nuda*

Cinara pilicornis*

Cinara pinea*

Cinara pini*

Formica (Serviformica) lemani Bondroit, 1917

Cinara pinea*

Cinara pini*

Formica (Serviformica) clara Forel, 1886

Cinara juniperi* 


\section{Index to diagnoses and figures of aphid species}

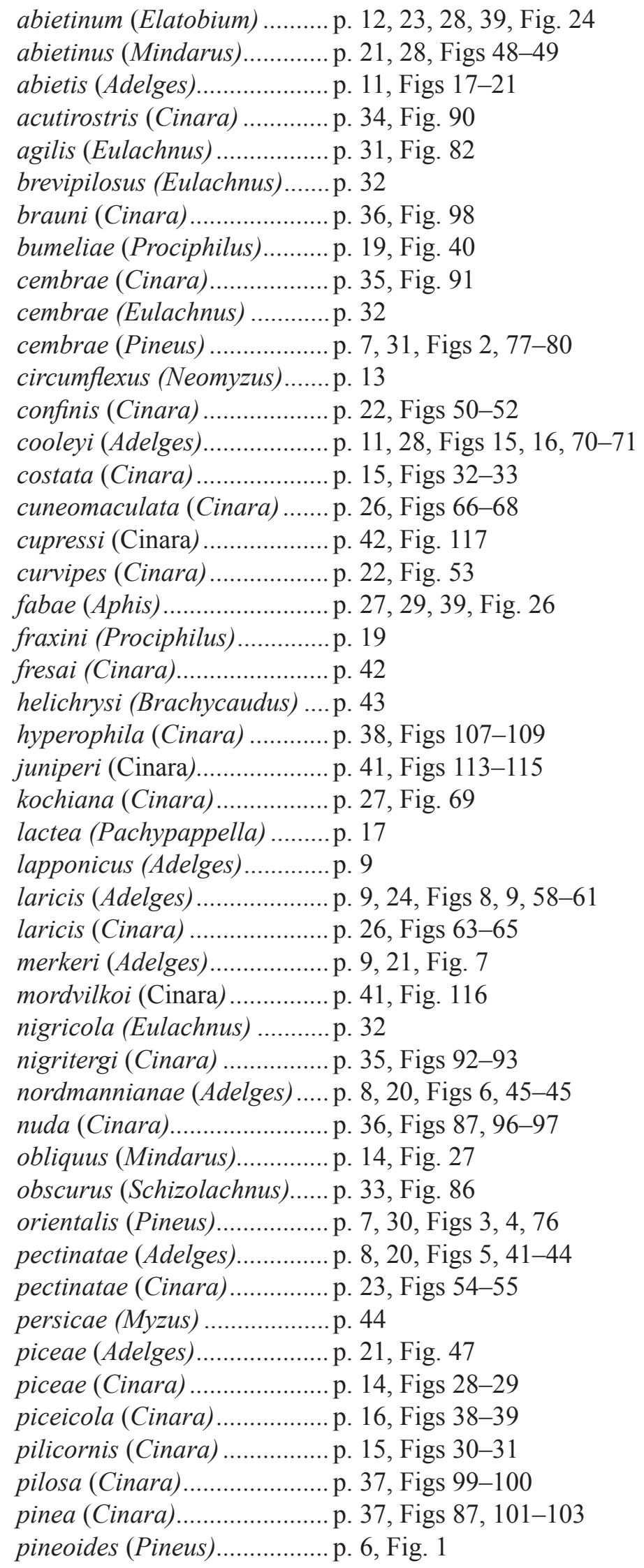




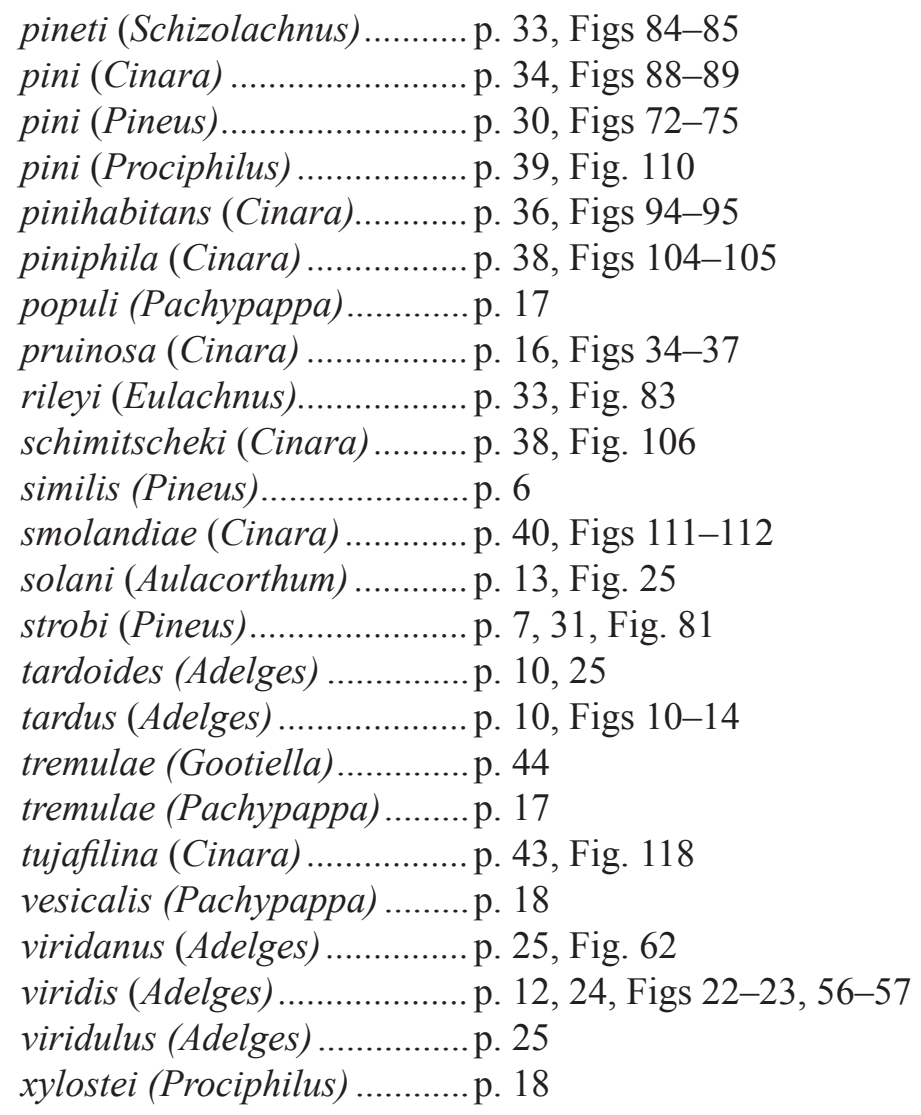

